\title{
Parametric Bose-Hubbard Hamiltonians: Quantum Dissipation, Irreversibility, and Pumping
}

\author{
Dissertation
}

zur Erlangung des Doktorgrades

der Mathematisch-Naturwissenschaftlichen Fakultäten der Georg-August-Universität zu Göttingen

vorgelegt von

\author{
Moritz Hiller \\ aus Göttingen
}

Göttingen 2007 
Referent:

Korreferent:
Prof. Dr. Theo Geisel

Prof. Dr. Kurt Schönhammer

Tag der mündlichen Prüfung: 19. Dezember 2007 


\section{Contents}

$\begin{array}{ll}\text { 1. Overview } & 1\end{array}$

2. Interacting Bosons on a Lattice and the Bose-Hubbard Hamiltonian 3

2.1. Optical lattices . . . . . . . . . . . . . . . . . . 6

2.2. Quantum description: The Bose-Hubbard Hamiltonian . . . . . . . . . . . 7

2.2.1. Derivation from second quantization . . . . . . . . . . 8

2.2.2. Validity of the local-mode approximation . . . . . . . . . . . 11

2.3. Semiclassical limit of the Bose-Hubbard Hamiltonian . . . . . . . . . . . . 11

2.3.1. The discrete nonlinear Schrödinger equation . . . . . . . . . . . 13

2.4. Mean field description: The Gross-Pitaevskii equation . . . . . . . . . . . . . 13

2.5. Applications of the Bose-Hubbard Hamiltonian . . . . . . . . . . . . 15

2.6. The Bose-Hubbard trimer . . . . . . . . . . . . . . . . . . . 16

2.6.1. Fock-space representation of the BHH . . . . . . . . . . 16

2.6.2. Hilbert-space of the symmetric BHH trimer . . . . . . . . . . . . 18

2.6.3. Classical dynamics . . . . . . . . . . . . . . . 18

3. Parametric Bose-Hubbard Hamiltonians: Spectra and Eigenfunctions 21

3.1. Statistical properties of the BHH: Spectra and bandprofile . . . . . . . . . . 22

3.1.1. Energy levels . . . . . . . . . . . . . . . . . 24

3.1.2. The bandprofile . . . . . . . . . . . . . . 26

3.1.3. Distribution of couplings . . . . . . . . . . . . . . . 29

3.2. RMT modeling . . . . . . . . . . . . . . . . . 31

3.3. Parametric evolution of eigenfunctions . . . . . . . . . 32

3.4. Approximations for the profile $P(n \mid m) \ldots \ldots \ldots 33$

3.5. Classical profile and quantum classical correspondence . . . . . . . . . . . 36

3.6. The $P(n \mid m)$ for the Bose-Hubbard Hamiltonian . . . . . . . . . . . . . . 38

3.6.1. The perturbative regimes . . . . . . . . . . . . . . 39

3.6.2. The non-perturbative regime and detailed quantum-classical correspondence . . . . . . . . . . . . . . . 42

3.7. Conclusions . . . . . . . . . . . . . . . . . . . 43

4. Wavepacket Dynamics in Energy Space 45

4.1. Preliminary considerations and object of the study . . . . . . . . . . . . . 46

4.1.1. Measures of the evolving distribution $P_{t}\left(n \mid n_{0}\right) \ldots \ldots$. . . . . 47

4.2. Linear response theory . . . . . . . . . . . . . . . . . . 48 
4.2.1. The energy spreading $\delta E(t) \ldots \ldots \ldots \ldots$

4.2.2. Quantum LRT derivation for $\mathcal{P}(t) \ldots \ldots \ldots$

4.2.3. The survival probability and the $\operatorname{LDoS} \ldots \ldots \ldots$. . . . . . . 53

4.3. Wavepacket dynamics of cold bosons on an optical lattice . . . . . . 53

4.3.1. Classical dynamics . . . . . . . . . . . . . . . . 55

4.3.2. Quantum energy spreading ............. 56

4.3.3. The survival probability $\mathcal{P}(t) \ldots \ldots \ldots \ldots$

4.3.4. Detailed quantum-classical correspondence . . . . . . . . . . 60

4.3.5. The dynamics of the IRMT model . . . . . . . . . . . . . . . 62

4.4. Quantum self-trapping in the Bose-Hubbard Hamiltonian . . . . . . . . . 64

4.5. Conclusions . . . . . . . . . . . . . . . . . . . 67

5. Fidelity 69

5.1. Fidelity and decoherence . . . . . . . . . . . . . . . . 70

5.1.1. Static bath . . . . . . . . . . . . . . 72

5.1.2. Dynamical bath . . . . . . . . . . . . . 73

5.2. Fidelity and quantum irreversibility . . . . . . . . . . . . . . 74

5.3. Fidelity of cold atoms in an optical lattice: Theoretical background . . . . . 76

5.4. Numerical analysis of the fidelity . . . . . . . . . . . . . . . . 80

5.4.1. Fidelity decay before the first revival . . . . . . . . . . . . 80

5.4.2. Fidelity echoes . . . . . . . . . . . . . . . . . 84

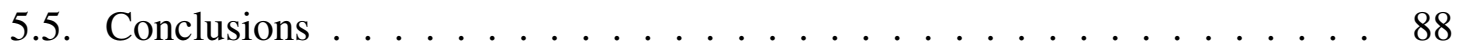

6. Quantum Pumping $\quad 91$

6.1. Operating an atom pump in a closed system: The BHH trimer model . . . . 92

6.2. Adiabatic theory . . . . . . . . . . . . . . . . . . . . . 94

6.3. Controlled atom current in the Bose-Hubbard trimer . . . . . . . . . . . . . 94

6.3.1. Two-orbital approximation . . . . . . . . . . . . . . 95

6.3.2. Evolution of energy levels . . . . . . . . . . . . . . 96

6.3.3. Two-orbital approximation for the $\mathrm{BHH}$ trimer . . . . . . . . . 998 98

6.3.4. Regimes . . . . . . . . . . . . . . . . 99

6.4. Conclusions . . . . . . . . . . . . . . . . . 103

7. Bose-Hubbard Dimer with Dissipation 105

7.1. Effective Hamiltonian modeling . . . . . . . . . . . . . . . 106

7.2. Classical modeling: DNLS with a sink . . . . . . . . . . . . . . 107

7.3. Resonance widths of the BHH dimer . . . . . . . . . . . . . . . . . . . 109

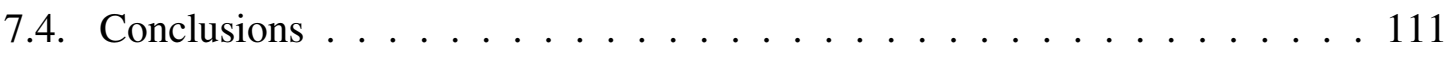

$\begin{array}{ll}\text { 8. Conclusions } & 113\end{array}$

A. Level Spacing Statistics and the Semiclassical Bandprofile 115 
A.1. Level spacing statistics of Gaussian ensembles . . . . . . . . . . . . . 115

A.1.1. Symmetry and block matrix representation . . . . . . . . . 120

A.1.2. Universality classes . . . . . . . . . . . . . . . . . . . . . 122

A.1.3. Unfolding spectra . . . . . . . . . . . . . . . . 125

A.2. Semiclassical bandprofile . . . . . . . . . . . . . . 125

B. Adiabatic Pumping and Transport in a Two-level System 129

B.1. Adiabatic pumping . . . . . . . . . . . . . . . . . . . . . 129

B.1.1. The adiabatic equation and the geometric conductance . . . . . . 130

B.2. Transport in a two-site system . . . . . . . . . . . . . . . . 132

$\begin{array}{ll}\text { Bibliography } & 135\end{array}$

$\begin{array}{ll}\text { Acknowledgments } & 151\end{array}$ 


\section{Overview}

While the physics of the previous century is mainly characterized by great advances in the understanding of the properties of single particle systems, recent experimental developments have placed the effects of interactions at the top of the current research agenda. One of the most fascinating achievements was the realization of Bose-Einstein condensation of ultra-cold atoms in optical lattices (OL) $[7,125,47,159]$ and the creation of "atom chips" $[90,108,160,176]$ which have been suggested as potential building blocks for quantum information processing [184] while at the same time they allow for novel, concrete applications of quantum mechanics such as atom interferometers [10, 187, 205] and lasers $[149,11,7,107,159]$, atom diodes and transistors $[150,189,196]$. The precise tailoring and manipulation of OLs, on the other hand, has allowed us to investigate complex solidstate phenomena, such as the Mott-insulator to superfluid transition [102], the Josephson effect [47], the atom blockade phenomenon in quantum-dot-like potentials [43], Anderson localization [9, 144, 91, 186, 51], and Bose-glass transitions [68]. In fact, it is envisioned that the emerging field of "atomtronics", i.e. the atom analog of electronics materials and circuits, will be able to provide nanoscale devices of unprecedented quality compared to the solid-state ones where imperfections and decoherence quickly destroy the delicate quantum effects. Finally, interacting bosonic systems - having a well-defined classical limit - provide an excellent playground to address fundamental questions related to the advancement of classical, semiclassical, and statistical methods.

Among all the exciting issues raised in the framework of interacting Bose systems, this thesis focuses on studying their response/dynamical evolution caused by an external driving field and on their transport and decay properties. The work is structured as follows:

- In Chapter 2 we set up the physical and mathematical framework for the description of interacting bosons on a lattice. After a brief introduction to the basic concepts of Bose-Einstein condensation, and how the condensate can be manipulated using optical lattices, we derive the Bose-Hubbard Hamiltonian (BHH) which is the paradigm model as far as the quantum description is concerned. Then the semiclassical limit of the BHH - the discrete nonlinear Schrödinger equation (DNLS) - as well as the mean-field (classical) description, the so-called Gross-Pitaevskii equation (GPE), is discussed. We give an overview of the relevant literature and of the various physical systems that are captured by the BHH including, among others, arrays of Josephson junctions and bond vibrations in small molecules. The chapter ends with a detailed description of the $\mathrm{BHH}$ trimer, a three-site ring-lattice which is the main model used in this thesis. 
- In (ultra-)cold atom-optics experiments, an effective potential for the atoms is created using lasers and magnetic fields. By time-varying the fields, a driving force is generated. Driven systems are described by parametric Hamiltonians, a fundamental concept in physics, which we introduce in Chapter 3. We consider a family of chaotic Bose-Hubbard Hamiltonians parameterized by the coupling strength $k$ between neighboring sites. As $k$ is increased the eigenstates undergo changes, reflected in the structure of the local density of states (LDoS). We analyze [112, 115] these changes, both numerically and analytically, using perturbative and semiclassical methods.

- The study and development of a theory for the quantum evolution of driven interacting bosonic systems with underlying classical chaotic dynamics is still lacking. We study the resulting quantum dynamics [116] (Chapter 4) and the irreversibility of the quantum motion [31] (Chapter 5), also used in the framework of "fidelity" studies in quantum computation.

- In Chapter 6 we initiate the study of quantum pumping/stirring in BECs $[113,114]$ with the aim to identify optimal pumping cycles and propose such a device in order to probe the interatomic interactions. The induced circulating atomic current is expected to be extremely accurate, and would open the way to various applications, either as a new metrological standard, or as a component of a new type of quantum information/ processing device.

- The interplay of intrinsic dynamics with coupling to the continuum, radiation fields, or to any other external influence, such as measurement, is an important subject for various branches of modern physics that boosted the research on open systems. In Chapter 7 we investigate the structure of the resonance widths [117] of a BoseHubbard dimer which is coupled to the continuum at one of the sites using an effective non-Hermitian Hamiltonian formalism.

Throughout this thesis we will approach quantum dynamics, decoherence, transport, and decay in interacting bosonic systems from a fundamental perspective, namely through the close interplay and exchange of ideas and techniques developed in areas as diverse as statistical, mathematical (nonlinear dynamics and wave chaos), atomic and solid state physics. At the core of this approach are tools of fundamental (e.g. wave/quantum chaos) and applied (e.g. solid-state) physics and mathematics employed in the context of the above cross-fertilization. On the one side, these tools are further deepened and extended, and on the other side, applied to the analysis of specific problems of great theoretical as well as technological interest. Underlying these problems is the fundamental question of understanding, at the quantum mechanical level, the traces of classical complex dynamics in the transport properties of (ultra-)cold atom devices. The possibility of manipulating such devices holds promise for application in interferometry, microscopy, atom lithography and quantum information processing. 


\section{Interacting Bosons on a Lattice and the Bose-Hubbard Hamiltonian}

In 1924, S. N. Bose [35] established the quantum statistics of non-interacting particles and derived Planck's radiation law by assuming that each quantum state can be occupied by an arbitrary number of photons. One year later, Einstein applied this concept to an ideal gas of identical atoms and predicted a new kind of phase transition [78, 79], known as the Bose-Einstein condensation. As illustrated in Fig. 2.1, the principle behind behind BoseEinstein condensation is that below a critical temperature $T_{c}$, a macroscopic fraction of atoms "condenses" into the ground state of the system. In other words, as the temperature $T$ is decreased, the thermal de-Broglie wave length, which scales as $T^{-1 / 2}$, is increased and at the critical point becomes comparable to the mean inter-particle distance. Therefore, the wave functions of the particles are sufficiently extended such that their overlap leads to a phase space density larger than unity, thus forming a Bose-Einstein condensate (BEC) as shown in Fig. 2.2. The otherwise intricate many-body wave function $\Psi$ then reduces to a product of $N$ identical single-particle ground state wavefunctions - in other words, all the atoms in the BEC are oscillating in unison - and can be represented by a single order parameter, the macroscopic condensate wave function $\psi$.

Despite the discovery of several phenomena which invoke the concept of Bose-Einstein condensation, notably superfluidity, it was only in 1995 that BEC was observed in its "ideal" form in a cloud of cold alkali atoms by the groups of Wieman/Cornell [8] and Ketterle [69] all of whom were awarded a Nobel prize. ${ }^{1}$ Though there were many obstacles in creating a Bose-Einstein condensate, the main one [153] was to cool the particles to temperatures close to absolute zero in order to achieve the necessary phase space density. A major step towards overcoming this obstacle was the realization of laser cooling of atoms, a technique which was proposed by the Nobel laureates W. D. Phillips, S. Chu, and C. Cohen-Tannoudji [62] in the 1980's. The principle of laser cooling is that due to the Doppler effect the atoms absorb light at a different rate depending on whether they are moving away from or towards the laser. The resulting kick in momentum can then be employed to slow down the atoms. Laser cooling led to temperatures on the order of a few hundred microkelvin which is nevertheless is too high to create a BEC. ${ }^{2}$ After the atoms are laser-cooled, "evaporative cooling" techniques are used: the pre-cooled atom cloud is kept in a magnetic dipole trap when the potential is slightly decreased. Thus, only the most energetic particles escape, removing more than the average energy (much like steam evaporating from a cup of hot coffee). Once

\footnotetext{
${ }^{1}$ Good introductory and overview articles on BEC include [67, 141, 133, 12, 168].

${ }^{2}$ Actually, the temperatures attained were below the theoretical predictions because sub-Doppler effects due to the pumping force were neglected. For a recent review on laser cooling see, for example, Ref. [133].
} 


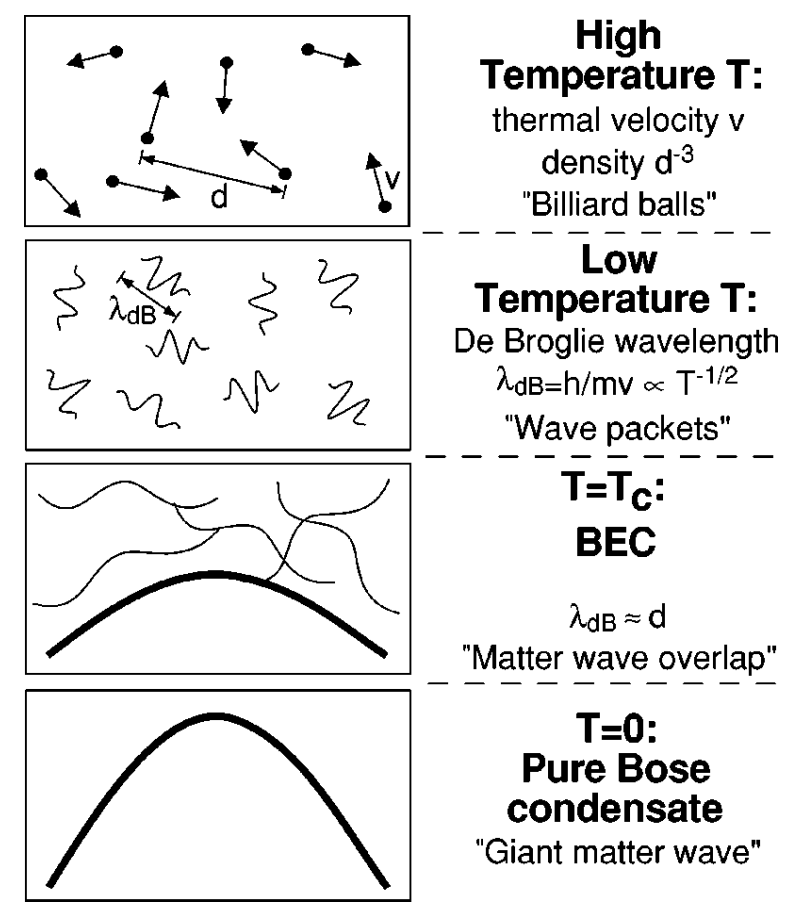

Figure 2.1.: Criterion for Bose-Einstein condensation. At high temperatures, a weakly interacting gas can be treated as a system of "billiard balls". In a simplified quantum description, the atoms can be regarded as wave packets with an extension of their de-Broglie wavelength $\lambda_{\mathrm{dB}}=\left(2 \pi \hbar^{2} / m k_{B} T\right)^{1 / 2}$, where $m$ is the mass of the particle, $k_{B}$ is the Boltzmann factor and $T$ is the temperature. At the BEC transition temperature $T_{c}, \lambda_{\mathrm{dB}}$ becomes comparable to the distance between atoms, and a Bose condensate forms. As the temperature approaches zero, the thermal cloud disappears, leaving a pure Bose condensate. Figure taken from [133].

the remaining atoms have re-thermalized to a reduced temperature, the trapping potential is repeatedly lowered, leading to a successive cooling of the particles and, finally, to the creation of a BEC at temperatures of several nanokelvin. It's a legitimate question to ask why the atomic gas doesn't liquefy or even solidify at these low temperatures? The answer is that the particle density is kept very low (typically $10^{14-16}$ particles $/ \mathrm{cm}^{3}$ ) throughout the cooling process. As a consequence, three-body collisions rarely occur. Thus, the interatomic interactions are dominated by two-body collisions which can be described by $s$-wave scattering [67].

Creating the BEC, however, is only the first step. Next, its properties must be explored. Although a multitude of fascinating experiments on ultra-cold atoms have been performed, BECs in (periodic) lattices are exceptionally interesting. On the one hand, these systems allow for the design of powerful nanoscale devices ("atomtronics"); on the other hand, bosons on lattices resemble crystals. Due to the unprecedented degree of control and precision as far as both the lattice geometry and the manipulation of the atomic cloud is concerned these "artificial crystals" constitute an ideal framework to study solid-state and mesoscopic 


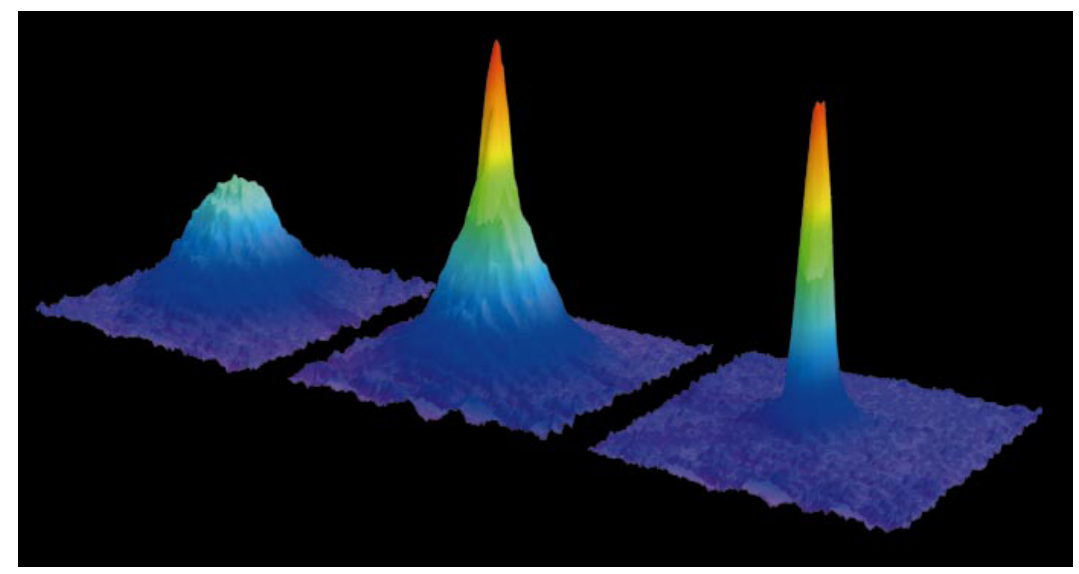

Figure 2.2.: Observation of Bose-Einstein condensation by absorption imaging. Shown is absorption vs two spatial dimensions. The Bose-Einstein condensate is characterized by its slow expansion observed after $6 \mathrm{~ms}$ time of flight. The left picture shows an expanding cloud cooled to just above the transition point; middle: just after the condensate appeared; right: after further evaporative cooling has left an almost pure condensate. The total number of atoms at the phase transition is about $7 \times 10^{5}$, the temperature at the transition point is $2 \mu \mathrm{K}$. Figure taken from [133].

physics, including fundamental questions on how the inter-particle interactions affect the behavior of the system.

Recent advances in micro-trap technology [160, 108, 176, 130] seem to be the most promising candidates for the realization of lattices consisting only of a few sites. In this technique, a magnetic potential is created by wires located on micro-chips. The condensate is either created directly in those traps $[160,108]$ or moved there after the evaporative cooling stage [105]. Due to the miniaturization these traps have also been suggested as potential building blocks for quantum information devices. However, to date the standard way of trapping atoms is using optical lattices which we are going to discuss in the following section.

In our study we will employ both a purely quantum and a semiclassical description of the bosonic systems. With respect to the former we utilize the Bose-Hubbard Hamiltonian $(\mathrm{BHH})$, one of the paradigm models concerning the quantum treatment of interacting bosons on (small) lattices. The BHH captures the physics of various bosonic systems and therefore the obtained results are not limited to Bose-Einstein condensates but should also be applicable to bond excitations in small molecules or Josephson Junctions. In the semiclassical limit the BHH reduces to the so-called discrete nonlinear Schrödinger equation (DNLS). By using both quantum and semiclassical descriptions we will later identify traces of quantum-classical correspondence.

In this chapter we set up the physical and mathematical framework for the description of interacting bosons on a lattice. We start with a brief introduction on how a Bose-Einstein condensate can be manipulated using optical potentials, a technique which has been mastered to date by over fifty experimental groups worldwide [204], making the BEC one of 
the best controlled many-body systems. In Section 2.2, the Bose-Hubbard Hamiltonian is derived starting from second quantization. After discussing its validity, we show how to approach the semiclassical limit of the BHH model leading to the DNLS in Section 2.3. Although not used in this work, we also briefly present for the sake of completeness the celebrated Gross-Pitaevskii equation (GPE), a mean-field (classical) description of BoseEinstein condensates which in the appropriate limit reduces to the DNLS. In Section 2.5, we discuss the various systems that are captured by the BHH including an overview of the relevant literature. The chapter ends with a detailed description of the $\mathrm{BHH}$ trimer, a three-site ring-lattice, which is the main model used in this work.

\subsection{Optical lattices}

During the evolution of the laser cooling techniques it became clear that the interference patterns resulting from the laser beams shining on the atom cloud effectively created a three dimensional egg carton, i.e. a lattice potential, for the atoms. This results from the AC Stark shift as we explain below and led, for example, to the creation of artificial crystals where the atoms are bound by light. In contrast to solid-state systems, these lattices can be made largely free of defects and can be easily manipulated in the experiment. ${ }^{3}$ Consequently, optical lattices are currently one of the leading system models for interacting bosons in periodic potentials. Therefore, they are especially well suited to experimentally realize the studies proposed here. In the following we will elaborate a bit more on how optical lattices are created and manipulated.

The interaction of neutral atoms with an electromagnetic field of frequency $\omega_{L}$ can be split in a conservative and a dissipative part. The latter results from the absorption of a photon from the field which is spontaneously emitted (i.e. scattered in a random direction) and leads to a net momentum kick in the direction of the laser. This mechanism is also used for laser cooling. The rate of the scattering process $\Gamma_{\text {scatt }}$ grows linearly with the peak intensity of the light $I_{p}$ and is inversely proportional to the detuning $\omega_{0}-\omega_{L}$ of the laser squared [61]

$$
\Gamma_{\text {scatt }}=\frac{I_{p}}{\left(\omega_{0}-\omega_{L}\right)^{2}},
$$

where $\omega_{0}$ is the transition frequency of the atom at which the photon is absorbed. The conservative part, on the other hand, results from the electric dipole induced in the atom by the electric component of the field. The electric dipole oscillates at the driving frequency $\omega_{L}$ of the laser light and its interaction with the electric field $E(t)$ induces an energy shift $\Delta E$ (the AC Stark shift) in the atomic energy level

$$
\Delta E=-\frac{1}{2} \alpha\left(\omega_{L}\right)\left\langle E^{2}(t)\right\rangle,
$$

\footnotetext{
${ }^{3}$ For a recent overview of optical lattices see for example Morsch et al. [153] and references therein.
} 
where $\alpha$ is the polarizability of the atomic level, exhibiting a resonance at $\omega_{0}$ and brackets $\langle\cdot\rangle$ denote a cycle average. If the detuning of the laser is negative $\omega_{L}<\omega_{0}$ ("red-detuned") then the induced dipole $D=\alpha\left(\omega_{L}\right) E$ is in phase with the electric field. Therefore, the potential energy is minimal when the intensity of the laser is maximal and the atoms are attracted to the bright spots. In the opposite case of a blue-detuned laser $\left(\omega_{L}>\omega_{0}\right)$ the atoms are attracted by the dark spots in the field. This is the experimentally favorable situation since the scattering rate $\Gamma_{\text {scatt }}$ effectively decreases with the decreasing light intensity. The depth $V_{0}$ of the optical trap is [153]

$$
V_{0} \propto \frac{I_{p}}{\omega_{0}-\omega_{L}} .
$$

Accordingly, in order to have a conservative potential one has to work at the largest detuning possible since then the dissipative part given by (2.1) can be neglected.

At this point the atoms are radially confined to the waist size of the laser beam but there is not lattice yet. The simplest way to create a periodic optical potential is to make two laser beams counter-propagate and let them interfere. For a wavelength $\lambda_{L}$ this results in a potential of the form

$$
V(x)=V_{0} \cos ^{2}(\pi x / d),
$$

where $d=\lambda_{L} / 2$ is the distance between two minima in the direction of the laser beam. In practice, such a lattice can be created, for example, by retro-reflecting a laser beam and inserting an opto-acoustical modulator. This device allows for fast (less than a microsecond) and precise control of the laser intensity and also introduces a shift of the laser frequency of tens of $\mathrm{MHz}$ [153]. Alternatively, one can use two phase-coherent beams and introduce a frequency shift between them. As a consequence the lattice is not stationary but moving and by increasing the shift one can even create accelerated optical lattices.

After the condensate has evolved on the lattice, the next step is to measure the atomic cloud. A popular technique is time of flight measurements. The trapping potential is switched off and a resonant laser is shone on the atoms from above while a CCD camera takes an image of the light distribution from below the condensate. Since this procedure destroys the condensate, a time-resolved experiment requires repetitive measurements, starting each time with the same initial conditions. This is not an obstacle since the of cooling of the atoms can be achieved within few seconds while the condensates can exist in the traps up to the order of several minutes [141, 12].

\subsection{Quantum description of cold bosons on a lattice: The Bose-Hubbard Hamiltonian}

We now turn to the mathematical description of (ultra-)cold bosons loaded on a lattice. Here we derive the Bose-Hubbard Hamiltonian (BHH), the simplest non-trivial quantum 
model that takes into account the competition between the interaction energy and the kinetic energy of the system. Then we discuss the BHH's validity.

\subsubsection{Derivation from second quantization}

As mentioned above, the particle density in the atomic cloud of a BEC is extremely low, hence three-body collisions are rare events. Accordingly, the many-body Hamiltonian describing $N$ interacting bosons confined by an external potential is given in second quantization by $[125,67]$

$$
\begin{aligned}
\hat{H}= & \int \mathrm{d} \mathbf{r} \hat{\Psi}^{\dagger}(\mathbf{r})\left[-\frac{\hbar^{2}}{2 m} \nabla^{2}+V_{\mathrm{lat}}(\mathbf{r})+V_{\mathrm{ext}}(\mathbf{r})\right] \hat{\Psi}(\mathbf{r}) \\
& +\frac{1}{2} \iint \mathrm{d} \mathbf{r} \mathrm{d} \mathbf{r}^{\prime} \hat{\Psi}^{\dagger}(\mathbf{r}) \hat{\Psi}^{\dagger}\left(\mathbf{r}^{\prime}\right) V\left(\mathbf{r}-\mathbf{r}^{\prime}\right) \hat{\Psi}\left(\mathbf{r}^{\prime}\right) \hat{\Psi}(\mathbf{r})
\end{aligned}
$$

where $\hat{\Psi}(\mathbf{r})$ and $\hat{\Psi}^{\dagger}(\mathbf{r})$ are the bosonic field operators that annihilate and create a particle at the position $\mathbf{r}$ respectively and $V\left(\mathbf{r}-\mathbf{r}^{\prime}\right)$ is the two-body inter-atomic potential. The term $V_{\text {lat }}(\mathbf{r})$ describes the (optical) lattice potential while $V_{\text {ext }}(\mathbf{r})$ accounts for a possibly present additional potential which is slowly varying along the lattice like the magnetic trap used for the evaporative cooling. Due to the extremely low temperatures (typically several nanokelvin) the predominant inter-atomic interaction results from $s$-wave scattering. Since also the particle density (and hence the mean inter-atomic distance) is very low we can approximate the otherwise complicated two-body interaction potential $V\left(\mathbf{r}-\mathbf{r}^{\prime}\right)$ with a delta-like contact-potential [141]

$$
V\left(\mathbf{r}-\mathbf{r}^{\prime}\right) \approx \frac{4 \pi a_{s} \hbar^{2}}{m} \times \delta\left(\mathbf{r}-\mathbf{r}^{\prime}\right),
$$

where $a_{s}$ is the $s$-wave scattering length and $m$ is the atomic mass. Even with this simplified potential $V\left(\mathbf{r}-\mathbf{r}^{\prime}\right)$ solving (2.5) is impractical if not impossible but we can use the fact that the underlying potential is periodic $V_{\text {lat }}(\mathbf{r})=V_{\text {lat }}(\mathbf{r}+\mathbf{d})$ with $\mathbf{d}$ being the lattice vector: The eigenstates of a single atom moving in a potential $V_{\text {lat }}(\mathbf{r})$, would be the well-known Bloch functions $\phi_{\mathbf{q}, n}(\mathbf{r})=e^{i \mathbf{q r}} u_{\mathbf{q} . n}(\mathbf{r})$, where $u_{\mathbf{q}, n}(\mathbf{r}+\mathbf{d})=u_{\mathbf{q}, n}(\mathbf{r})$ and $\mathbf{q}$ is the so-called quasimomentum. The presence of the periodic potential leads to the formation of the socalled Bloch bands in the energy spectrum which are labeled by the sub-index $n$. As in a substantial part of the experimental studies $[47,202,159,102,48]$ we consider deep lattices in this work. Therefore, it is useful to work in a basis where the eigenfunctions are localized at the sites $i$. Such a basis is given by the Wannier-functions

$$
w_{n}\left(\mathbf{r}-\mathbf{r}_{i}\right)=\frac{1}{\sqrt{f}} \sum_{\mathbf{q}} e^{-i \mathbf{q} \mathbf{r}_{i}} \phi_{\mathbf{q}, n}(\mathbf{r}),
$$

which are obtained via a uniform transformation from the Bloch basis. Above, the summation is done over the quasi-momentum in the first Brillouin zone and $f$ denotes the number 
of lattice sites. ${ }^{4}$ If the lattice is deep enough such that the chemical potential is too small to excite states outside the first Bloch band $[151,125]$ we can expand the field operators $\hat{\Psi}$ of the above Hamiltonian (2.5) in the local modes $w_{n}\left(\mathbf{r}-\mathbf{r}_{i}\right)$ of the wells keeping only the states belonging to the lowest band

$$
\hat{\Psi}(\mathbf{r})=\sum_{i=1}^{f} \hat{b}_{i} w_{0}\left(\mathbf{r}-\mathbf{r}_{i}\right),
$$

where $\hat{b}_{i}$ annihilates a boson at site $i$ and $f$ is the size of the lattice. The Hamiltonian (2.5) then reduces to the Bose-Hubbard Hamiltonian [125, 151]

$$
\begin{aligned}
\hat{H} & =\sum_{i=1}^{f} v_{i} \hat{b}_{i}^{\dagger} \hat{b}_{i}+\frac{1}{2} \sum_{i=1}^{f} U_{i} \hat{b}_{i}^{\dagger} \hat{b}_{i}^{\dagger} \hat{b}_{i} \hat{b}_{i}-\sum_{\langle i, j\rangle} k_{i j} \hat{b}_{i}^{\dagger} \hat{b}_{j} \\
& =\sum_{i=1}^{f} v_{i} \hat{n}_{i}+\frac{1}{2} \sum_{i=1}^{f} U_{i} \hat{n}_{i}\left(\hat{n}_{i}-1\right)-\sum_{\langle i, j\rangle} k_{i j} \hat{b}_{i}^{\dagger} \hat{b}_{j},
\end{aligned}
$$

where $\langle i, j\rangle$ indicates summation over adjacent sites $j=i \pm 1$. In the second step we used the canonical commutation rules for the bosonic annihilation (creation) operators $\hat{b}_{i},\left(\hat{b}_{i}^{\dagger}\right)$

$$
\left[\hat{b}_{i}, \hat{b}_{j}^{\dagger}\right]=\delta_{i, j}
$$

and the definition of the number operators

$$
\hat{n}_{i}=\hat{b}_{i}^{\dagger} \hat{b}_{i}
$$

The parameters in Eq. (2.10) are

$$
\begin{aligned}
v_{i} & =\int \mathrm{d}^{3} r V_{\mathrm{ext}}(\mathbf{r})\left|w_{0}\left(\mathbf{r}-\mathbf{r}_{i}\right)\right|^{2} \\
U_{i} & =\frac{4 \pi a_{s} \hbar^{2}}{m} \int \mathrm{d}^{3} r\left|w_{0}\left(\mathbf{r}-\mathbf{r}_{i}\right)\right|^{4} \\
k_{i j} & =\int \mathrm{d}^{3} r w_{0}^{*}\left(\mathbf{r}-\mathbf{r}_{i}\right)\left[-\frac{\hbar^{2}}{2 m} \nabla^{2}+V_{\text {lat }}(\mathbf{r})\right] w_{0}\left(\mathbf{r}-\mathbf{r}_{j=i \pm 1}\right) .
\end{aligned}
$$

Here $v_{i}$ is the on-site potential at each lattice site, $U_{i}$ is the on-site interaction strength, ${ }^{5}$ and $k_{i j}$ parameterizes the coupling strength which accounts for the tunneling of particles between neighboring sites. ${ }^{6}$ Therefore, $k_{i j}$ is the proportionality factor for the kinetic energy.

\footnotetext{
${ }^{4}$ Here we are interested in one-dimensional lattices, but the derivation also applies to higher dimensions.

${ }^{5}$ The inverse of the integral in (2.14) is also referred to as the effective mode volume $V_{\text {eff }}^{-1}=$ $\int \mathrm{d}^{3} r\left|w_{0}\left(\mathbf{r}-\mathbf{r}_{i}\right)\right|^{4}$.

${ }^{6}$ Apart from Chapter 6 we will consider a setup where $U_{i}=U$ and $k_{i j}=k$.
} 
The BHH was originally conceived [153] to describe superfluid He in restricted geometries like porous media (see e.g. [88]) and later suggested by Jaksch et. al [125] as a description of BEC in optical lattices. Both works were focused on quantum phase transitions which is not the topic of this thesis but an important result that we mention here. In contrast to their classical analog, quantum phase transitions occur at $T=0$. In other words they are not driven by the temperature $T$ but result only from quantum (not thermal) fluctuations [181]. The phase transition in the Bose-Hubbard Hamiltonian (2.10) is a result of the competition between the interaction energy and the kinetic energy. Roughly speaking, the nonlinearity tries to localize the bosons, while the coupling tries to delocalize them. Consider the limit $U \gg k$ for an optical lattice with exactly one atom per well, i.e., a filling factor $\bar{n}=N / f=1$. The energy cost to move one atom in this case is $U$ and determines the energy gap to the first excited (i.e. conducting) state. If this energy is not provided by, say, an external potential that sufficiently tilts the lattice, this configuration is insulating (no atom current) and the system is in the so-called Mott-insulator state [88, 125]. In the other limit of $k \gg U$ one can neglect the interaction term. The resulting Hamiltonian is then diagonal in the Bloch basis [71] and all particles will be completely delocalized over the entire lattice. For vanishing on-site potentials $v_{i}=0$ the BEC ground state corresponds to a quasimomentum $q=0$. In contrast to the previous case the atoms form a superfluid. Thus, tilting the lattice even slightly will cause the bosons to move. As the parameter $U / k$ is changed from $\infty \rightarrow 0$ the BEC exhibits the so-called Mott-insulator to superfluid transition which was experimentally confirmed in a seminal paper of Greiner et al. [102]. We note that this transition is appreciable only for small integer filling factors $\bar{n}$. If the filling factor is non-integer then there is always one atom that can move. This is sufficient to ensure phase coherence between wells and hence the system is in the superfluid regime.

One of the advantages of realizing the $\mathrm{BHH}$ with optical lattices is that all parameters are accessible in the experiment. In other words, while the on-site potential $v_{i}$ is given by $V_{\text {ext }}\left(\mathbf{r}_{i}\right), U_{i}$ and $k_{i}$ are determined by the wavefunction $w_{0}\left(\mathbf{r}-\mathbf{r}_{i}\right)$ which depends on the lattice depth $V_{0}$, i.e. on the intensity of the interfering laser beams. For typical lattice configurations the tunneling strength decreases exponentially with the lattice depth $k \sim e^{-V_{0}}$, while the on-site interaction grows algebraically [177] $U \sim V_{0}^{D / 4}$ (here $D$ is the dimensionality of the lattice). Additionally, the scattering length $a_{s}$ can be tuned using Feshbach resonances [141] - by applying an additional magnetic field the hyperfine levels that determine the $s$-wave scattering are shifted. With this technique, the parameter $a_{s}$ can be changed over several orders of magnitude [119] including a change in sign. For negative scattering lengths $a_{s}$ the condensate becomes unstable above a certain boson number $N$ due to the attractive interaction. Unless stated otherwise, we consider only repulsive interactions, i.e. $a_{s}>0$. 


\subsubsection{Validity of the local-mode approximation}

In the derivation of the Bose-Hubbard Hamiltonian (2.10) we made several assumptions. We already discussed the approximation of the atom-atom interaction by a delta-like contact potential above. Next, we expanded the field operators $\hat{\Psi}$ in the single particle Wannier states of the lowest Bloch band. For this it is crucial that the chemical potential, the kinetic energy and the interaction energy are too low to excite states in the second Bloch band. Accordingly, the lattice must be very deep [67, 151,153] since this leads to a large energy gap between the bands. While the above parameters (2.13)-(2.15) can be readily evaluated numerically for a given lattice potential (2.4), qualitative insight in their dependence on $V_{0}$ is gained by a harmonic approximation around the potential minima (see, for example, Ref.[125]). Furthermore, the interaction energy has to be smaller than the single particle ground state energy $E_{0}$, otherwise the single particle wavefunction may be strongly modified by the interaction. Approximating the wavefunction with a Gaussian and taking a standard harmonic trap with a size of $10 \mu \mathrm{m}$ and a scattering length $a_{s}=5 \mathrm{~nm}$ one finds that the BHH model is valid for up to several hundred bosons [151] per trap. ${ }^{7}$

Moreover, deep lattices allow us to drop higher order terms when inserting Eq. (2.8) into (2.5). In the interaction we only took into account the on-site contribution, but neglected higher order terms of the type

$$
\int \mathrm{d}^{3} r\left|w_{0}\left(\mathbf{r}-\mathbf{r}_{i}\right)\right|^{2}\left|w_{0}\left(\mathbf{r}-\mathbf{r}_{\mathbf{j}}\right)\right|^{2} \approx 0
$$

Additionally, we made a tight-binding approximation with respect to the coupling strength $k$ and omitted terms that go beyond nearest-neighboring wells

$$
\int \mathrm{d}^{3} r w_{0}^{*}\left(\mathbf{r}-\mathbf{r}_{i}\right)\left[-\frac{\hbar^{2}}{2 m} \nabla^{2}+V_{\text {lat }}(\mathbf{r})\right] w_{0}\left(\mathbf{r}-\mathbf{r}_{i \pm 2,3, \ldots}\right) \approx 0 .
$$

It turns out $[151,125,63]$ that this is justified since these terms are usually two orders of magnitude smaller than the ones which were kept. Summarizing the above, the BHH is a good description for deep lattices and moderate boson numbers, which is the setup considered in this work.

\subsection{Semiclassical limit of the $\mathrm{BHH}$ and the discrete nonlinear Schrödinger equation}

One advantage of the bosonic systems described by the BHH (2.10) is that they have a well-defined semiclassical limit, which allows us to ask fundamental questions of quantumclassical correspondence (QCC). Naively, one might expect that the semiclassical limit of

\footnotetext{
${ }^{7}$ We note that for very large boson numbers and constant interaction strength $U$, one can apply the so-called Thomas-Fermi approximation (see e.g. Ref. [67]) which neglects the kinetic energy.
} 
the BHH simply corresponds to large particle numbers $N \gg 1$. However, the interaction strength $U$ also has to be taken into account appropriately in the limiting process $N \rightarrow \infty$. Formally, this can be seen if we define rescaled creation and annihilation operators

$$
\hat{c}_{i}^{(\dagger)}=\frac{1}{\sqrt{N}} \hat{b}_{i}^{(\dagger)} ; \quad \hat{\tilde{n}}_{i}=\frac{1}{N} \hat{n}_{i}
$$

leading to ${ }^{8}$

$$
\frac{\hat{H}}{N}=\sum_{i=1}^{f} v_{i} \hat{\tilde{n}}_{i}+\frac{U N}{2} \sum_{i=1}^{f} \hat{\tilde{n}}_{i}\left(\hat{\tilde{n}}_{i}-\frac{1}{N}\right)-k \sum_{\langle i, j\rangle} \hat{c}_{i}^{\dagger} \hat{c}_{j}
$$

The commutators

$$
\left[\hat{c}_{i}, \hat{c}_{j}^{\dagger}\right]=\frac{1}{N} \delta_{i, j}
$$

vanish for large particle numbers $N \gg 1$ and therefore one can treat the rescaled operators as $c$-numbers in the limit $N \rightarrow \infty$. At the same time, it is clear that the second term in (2.19) still depends on the particle number $N$. Hence, the nonlinear contribution would inevitably dominate for large $N$. Therefore we define

$$
\tilde{U}=U N ; \quad \lambda=\frac{k}{\tilde{U}}
$$

where $\tilde{U}$ is the effective nonlinearity and $\lambda$ is the ratio between kinetic and nonlinear potential energy. Keeping both $\tilde{U}$ and $\lambda$ constant as $N$ is increased, the classical Hamiltonian $\mathcal{H}$ is obtained using the Heisenberg relations

$$
\hat{c}_{j} \mapsto \sqrt{I_{j}} e^{i \varphi_{j}} ; \quad \hat{c}_{j}^{\dagger} \mapsto \sqrt{I_{j}} e^{-i \varphi_{j}},
$$

where $I_{j}$ is an action and $\varphi_{j}$ is the associated angle $\left(I_{j}, \varphi_{j} \in \mathbb{R}\right)$. Omitting the $1 / N$ term of the nonlinear part we then get

$$
\tilde{\mathcal{H}}=\frac{\mathcal{H}}{\tilde{U} N}=\sum_{i=1}^{f} \frac{v_{i}}{\tilde{U}} I_{i}+\frac{1}{2} \sum_{i=1}^{f} I_{i}^{2}-\lambda \sum_{\langle i, j\rangle} \sqrt{I_{i} I_{j}} e^{-i\left(\varphi_{i}-\varphi_{j}\right)}
$$

Using the canonical equations

$$
\dot{\varphi}_{j}=\partial_{\tilde{t}} \varphi_{j}=\frac{\partial \tilde{\mathcal{H}}}{\partial I_{j}} ; \quad \dot{I}_{j}=\partial_{\tilde{t}} I_{j}=-\frac{\partial \tilde{\mathcal{H}}}{\partial \varphi_{j}},
$$

with $\tilde{t}=\tilde{U} t$ being the rescaled time, the classical equations of motion are obtained from Eq. (2.23) to be

$$
\begin{aligned}
\dot{\varphi}_{j} & =\frac{v_{j}}{\tilde{U}}+I_{j}-\lambda \sqrt{\frac{I_{i}}{I_{j}}} \cos \left(\varphi_{j}-\varphi_{i}\right) \\
\dot{I}_{j} & =2 \lambda \sqrt{I_{i} I_{j}} \sin \left(\varphi_{j}-\varphi_{i}\right)
\end{aligned}
$$

\footnotetext{
${ }^{8}$ Here we consider the homogenous lattice $U_{i}=U$ and $k_{i j}=k$ but also heterogeneities can be taken into account by defining mean values $\bar{k}, \bar{U}$.
} 
The dimensionless ratio $\lambda=k / \tilde{U}[188,203,71,92,155]$ determines the dynamics of the classical Hamiltonian (2.23). For $\lambda \rightarrow 0$ the interaction term dominates and the system behaves as a set of uncoupled sites while for $\lambda \rightarrow \infty$ the kinetic term is the dominant one. We will discuss the classical dynamics in more detail in Subsection 2.6.3.

\subsubsection{The discrete nonlinear Schrödinger equation}

Alternatively, the classical Hamiltonian (2.23) is frequently expressed in complex amplitudes

$$
A_{j}=\sqrt{I_{j}} e^{i \varphi_{j}} .
$$

In other words, one replaces the bosonic operators $c_{j} \rightarrow A_{j}, c_{j}^{\dagger} \rightarrow A_{j}^{*}$ leading to the classical Hamiltonian

$$
\frac{\mathcal{H}_{\mathrm{DNLS}}}{N}=\sum_{i=1}^{f} v_{i}\left|A_{i}\right|^{2}+\frac{U N}{2} \sum_{i=1}^{f}\left|A_{i}\right|^{4}-k \sum_{\langle i, j\rangle} A_{i}^{*} A_{j} .
$$

The amplitudes $A_{j}^{(*)}$ are conjugate variables with respect to the Hamiltonian $i \mathcal{H}$ and the canonical equations read

$$
i \partial_{\tilde{t}} A_{j}=\frac{\partial \mathcal{H}}{\partial A_{j}^{*}} ; \quad-i \partial_{\tilde{t}} A_{j}^{*}=\frac{\partial \mathcal{H}}{\partial A_{j}}
$$

with the resulting equations of motion

$$
i \dot{A}_{j}(\tilde{t})=v_{i} A_{j}(\tilde{t})+U N\left|A_{j}(\tilde{t})\right|^{2} A_{j}(\tilde{t})-k \sum_{i \neq j} A_{i}(\tilde{t}) .
$$

The latter equation is usually referred to as the discrete nonlinear Schrödinger equation (DNLS) [75] and is one of the prototype models used in quantum chemistry to describe bond excitations of small molecules. In this context it represents a special case of the so-called discrete self-trapping equation (DST) [76], which has the same form as (2.29) but is not restricted to nearest-neighbor couplings. Under the appropriate discretization, the DNLS can also be recovered from the mean-field description of Bose-Einstein condensates, the (classical) Gross-Pitaevskii equation which we discuss in the next section.

\subsection{Mean field description of Bose-Einstein condensates: The Gross-Pitaevskii equation}

Another way to approach the full quantum problem given by Hamiltonian (2.5) is by using a mean field approach. ${ }^{9}$ The basic mean-field description of a dilute gas was originally

\footnotetext{
${ }^{9}$ See, for example, Ref. [67] for a detailed derivation.
} 
formulated by Bogoliubov [32] for the homogenous case of a BEC in a single trap. Its generalization to the case of time-dependent and non-uniform (due to the confinement) configurations of the atomic cloud is given by describing the field operators $\hat{\Psi}(\mathbf{r})$ in the Heisenberg representation by

$$
\hat{\Psi}(\mathbf{r}, t)=\psi(\mathbf{r}, t)+\delta \hat{\Psi}(\mathbf{r}, t) .
$$

Here $\psi(\mathbf{r}, t)$ is a complex function defined as the expectation value of the field operator, i.e., $\psi(\mathbf{r}, t)=\langle\hat{\Psi}(\mathbf{r}, t)\rangle$ and its modulus represents the condensate density through $n_{0}(\mathbf{r}, t)=|\psi(\mathbf{r}, t)|^{2}$. In contrast to the operator $\hat{\Psi}(\mathbf{r}, t), \psi(\mathbf{r}, t)$ is a classical field having the meaning of an order parameter and is often called the "macroscopic wave function of the condensate".

The above approximation (2.30) implicitly assumes that the number of atoms $N$ in the BEC is very large and hence one can neglect the quantum fluctuations which are characterized by $\delta \hat{\Psi}(\mathbf{r}, t)$ and are also referred to as the "quantum depletion" of the condensate. Accordingly, the mean-field ansatz is particularly useful for large atom numbers but becomes invalid if quantum fluctuations are important.

In order to derive the evolution of the condensate wave function $\psi(\mathbf{r}, t)$ one evaluates the Heisenberg equations for the field operators

$$
i \hbar \partial_{t} \hat{\Psi}(\mathbf{r}, t)=[\hat{\Psi}(\mathbf{r}, t), \hat{H}],
$$

where $\hat{H}$ is the many-body Hamiltonian (2.5), by substituting Eq. (2.30). This yields the celebrated Gross-Pitaevskii equation (GPE)

$$
i \hbar \partial_{t} \psi(\mathbf{r}, t)=\left[-\frac{\hbar^{2}}{2 m} \nabla^{2}+V_{\mathrm{lat}}(\mathbf{r})+V_{\mathrm{ext}}(\mathbf{r})+U|\psi(\mathbf{r}, t)|^{2}\right] \psi(\mathbf{r}, t)
$$

Gross [103, 104] and Pitaevskii[167] independently found the above Eq. (2.32) and based it on the assumptions that the numbers of atoms $N \gg 1$ in the BEC is much larger than one. ${ }^{10}$ The GPE and can be used to explore the macroscopic behavior of the system, characterized by variations of the order parameter over distances larger than the mean distance between atoms.

For deep lattices one can apply the same tight-binding approximation as in the quantum case (2.8), namely by expanding the condensate function $\psi(\mathbf{r}, t)$ in the basis of the Wannierfunctions $w_{0}\left(\mathbf{r}-\mathbf{r}_{i}\right)$ which are localized at the lattices site $j$ and correspond to the lowest lying orbital

$$
\psi(\mathbf{r}, t)=\sum_{i} A_{i}(t) w_{0}\left(\mathbf{r}-\mathbf{r}_{i}\right) .
$$

Keeping only the next-neighbor contributions to the coupling and the on-site contributions for the interactions one arrives again at the DNLS described by Eqs. (2.27) and (2.29).

\footnotetext{
${ }^{10}$ Since the derivation starts from Eq. (2.5) it is implicitly assumed, that the relevant inter-atomic interaction are two-body processes, characterized by the $s$-wave scattering length $a_{s}$.
} 

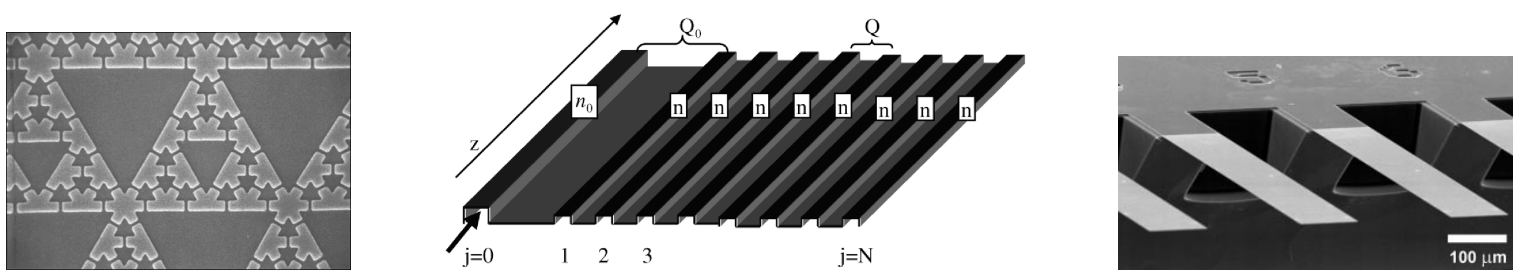

Figure 2.3.: Various physical systems captured by the BHH and DNLS (from left): Coupled Josephson Junctions with the geometry of periodically repeated Sierpinski gaskets, superconducting metal is $\mathrm{Pb}$ (clear) while the normal metal is $\mathrm{Cu}$ (dark) taken from [127]; array of coupled nonlinear waveguides taken from [134], array of coupled micro-cantilevers taken from [95].

\subsection{Applications of the Bose-Hubbard Hamiltonian}

The Bose-Hubbard Hamiltonian and its semiclassical limit, the DNLS, find applications in a variety of physical systems that we discuss here. We also present a brief overview of the basic literature while publications that are relevant to the specific problems treated in this thesis will be cited in the corresponding chapters.

A substantial part of the BHH literature concerns ultra-cold atoms in (optical) lattices, which results from the currently large momentum in the field of BEC. Since the exact quantum treatment is often limited due to computational restrictions many studies are using numerical methods like Monte-Carlo simulations [207] or renormalization group techniques [185] and the mean-field Gross-Pitaevskii equation. For a recent review see Ref. [153].

Another bosonic system captured by the BHH are Josephson Junctions arrays (JJA) like the one presented in the left panel of Fig. 2.3. In contrast to the neutral atoms in a BEC, here Cooper-pairs of two electrons form the (now charged) bosons. The predominant interaction described by $U$ is therefore the Coulomb repulsion while $k$ represents the Josephson energy $E_{J}$ (see for example Ref. [39] for a detailed description of JJA and the BHH). Findings include superconducting-insulator transitions or Josephson oscillations [81].

The BHH is also used in (quantum) chemistry where it describes bond excitations in small molecules [188] and biological polymers [82]. Here $k$ accounts for the electromagnetic and mechanical coupling of adjacent atoms in the molecule while $U$ represents the anharmonic softening of the bonds under extension [188, 21, 13]. The numerous studies include both semiclassical [76] as well as quantum [64, 182, 215, 49] treatment of the problem and for a recent review see Ref. [75]. Similarly, the BHH can be used to describe the nonlinear vibrations of micro-cantilever arrays [183] (see middle panel of Fig. 2.3) that were recently used together with BEC confined in magnetic micro-traps [201].

Finally, the field of nonlinear optics must be mentioned where the DNLS is used to describe propagation of light in arrays of nonlinear waveguides (see right panel of Fig. 2.3) such as linearly coupled, non-dispersive, single-mode Kerr fibers [87, 22]. For a recent review see Ref. [111]. 


\subsection{The Bose-Hubbard trimer}

In this section we describe in detail the model used mainly in thesis, which is a three-site ring-lattice, termed $\mathrm{BHH}$ trimer. We start with the quantum model and then turn to the classical dynamics.

\subsubsection{Fock-space representation of the $\mathrm{BHH}$}

As one readily verifies the Bose-Hubbard Hamiltonian (2.10) has two constants of motion [76, 21], namely the energy $E=H$ and the total number $N$ of particles

$$
N=\sum_{i=1}^{f} n_{i} .
$$

Therefore we can choose as a basis the Fock number states $\left|n_{1}, n_{2}, \ldots, n_{f}\right\rangle$, where the number of atoms in each site $n_{i}$ is well-defined

$$
\begin{array}{r}
\{\underbrace{\mid N, 0, \ldots, 0}_{f \text {-times }}\rangle,|N-1,1,0, \ldots, 0\rangle,|N-1,0,1,0, \ldots, 0\rangle, \ldots,|N-1,0, \ldots, 0,1\rangle, \\
|N-2,2,0, \ldots, 0\rangle,, \ldots,|N-2,1,1,0, \ldots, 0\rangle, \ldots,|0, \ldots, 0, N\rangle\}
\end{array}
$$

and the total number of particles is $N=$ const. The action of the annihilation (creation) operators $\hat{b}_{i}\left(\hat{b}_{i}^{\dagger}\right)$ on the Fock states is given by

$$
\begin{aligned}
\hat{b}_{i}\left|n_{1}, n_{2}, \ldots, n_{i}, \ldots, n_{f}\right\rangle & =\sqrt{n_{i}}\left|n_{1}, n_{2}, \ldots, n_{i}-1, \ldots, n_{f}\right\rangle \\
\hat{b}_{i}^{\dagger}\left|n_{1}, n_{2}, \ldots, n_{i}, \ldots, n_{f}\right\rangle & =\sqrt{n_{i}+1}\left|n_{1}, n_{2}, \ldots, n_{i}+1, \ldots, n_{f}\right\rangle .
\end{aligned}
$$

For vanishing coupling strength $k=0$, the BHH (2.10) is already diagonal in this basis. In the opposite limit $U=0$ the $\mathrm{BHH}$ can be diagonalized exactly by a unitary transformation to the Bloch eigenstate basis [71]. Using elementary combinatorial considerations of how to distribute $N$ indistinguishable particles over $f$ wells one finds that the dimension $\mathcal{N}$ of the corresponding Hilbert-space is possibly large but finite [71, 21]

$$
\mathcal{N}=\frac{(N+f-1) !}{N !(f-1) !} .
$$

On the one hand, this allows for a full quantum treatment of the problem, i.e. no truncation of the Hilbert-space is necessary. On the other hand, the rapid increase of the dimension $\mathcal{N}$ often restricts the study to systems of few sites and a small number $N$ of bosons due to computational limitations. ${ }^{11}$

\footnotetext{
${ }^{11}$ If exact diagonalization is not required, one can study larger systems by using numerical methods like Monte-Carlo simulations [207] or renormalization group techniques [185].
} 

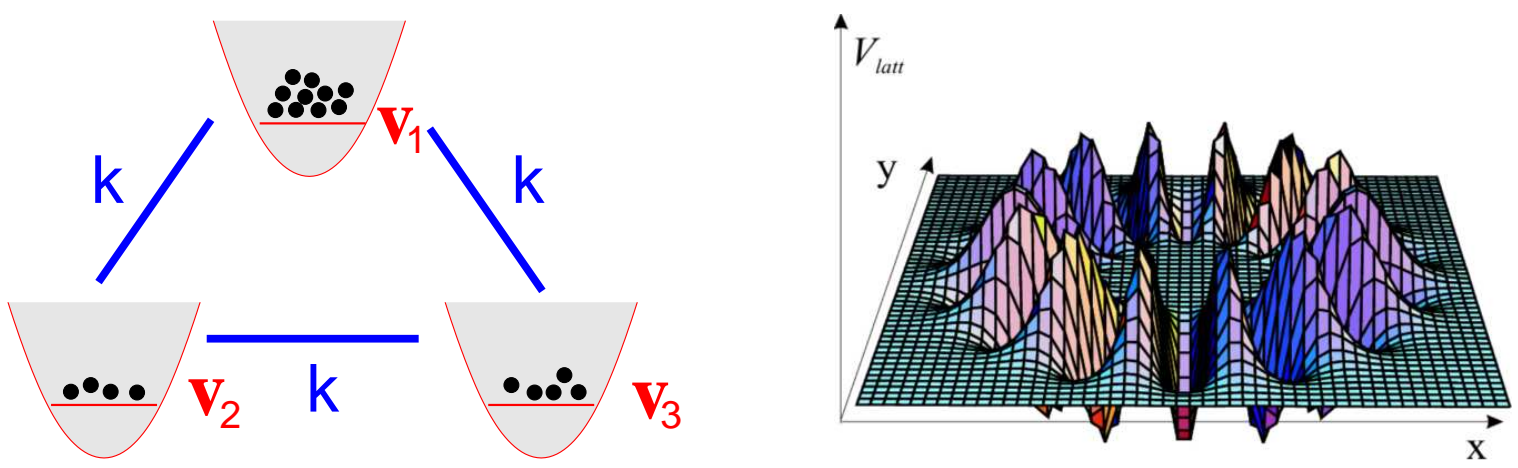

Figure 2.4.: Left: Schematic plot of the Bose-Hubbard trimer as a three-site ring-lattice. The coupling strength between sites is parameterized by $k_{i}=k$ while the on-site potential is $v_{i}=0$. The on-site interaction is given by $U_{i}=U$ (not shown). Right: The optical potential $V_{\text {lat }}$ resulting from the interference of a plain wave with an Laguerre-Gauss laser mode. Figure taken from [3].

In this context, the two-site system (dimer) has been analyzed thoroughly from both the semiclassical $[76,203]$ and the purely quantum viewpoint $[94,21,129]$. These investigations have revealed many interesting phenomena like the onset of $\pi$-phase oscillations, symmetry-breaking, and self-trapping of boson population, the latter being observed experimentally in Ref. [1].

Since the BHH has two constants of motion, the dimer - having two degrees of freedom - is classically integrable. Notwithstanding, the plethora of fascinating results adds to the motivation to go beyond the dimer and consider new scenarios where even richer dynamics should be observed. In this respect, the trimer opens new and exciting opportunities, since the addition of a third site leads to (classically) chaotic behavior. Chaoticity is abundant in nature, but more importantly leads to universal behavior and is therefore a major ingredient in the dynamical evolution. Consequently, the study of the trimer will pave the way for understanding longer (i.e. also classically chaotic) lattices.

In the rest of this thesis we will consider mainly a three-site ring-lattice as the one schematically depicted in the left panel of Fig. 2.4. Unless stated otherwise we will consider vanishing on-site potentials $v_{i}=0$. The couplings $k_{i j}$ between neighboring sites are assumed to be equal $k_{i j}=k$ which applies also to the on-site interaction $U_{i}=U$ (see Subsection 2.2.1 for a discussion of the parameters). From Eq. (2.10) we then obtain

$$
\hat{H}=\frac{U}{2} \sum_{i=1}^{3} \hat{n}_{i}\left(\hat{n}_{i}-1\right)-k \sum_{\langle i, j\rangle} b_{i}^{\dagger} b_{j} .
$$

Such ring-lattices are experimentally feasible with current optical methods where the optical potential is created by letting a plane wave interfere with the so-called LaguerreGauss laser modes as described in [3] (see right panel of Fig. 2.4) but also micro-traps $[160,108,176,130]$ are promising candidates for realizing these small systems. 


\subsubsection{Hilbert-space of the symmetric BHH trimer}

The 3-fold permutation symmetry of our trimer model (2.39) leads to a separation of the Hilbert-space in three independent subspaces [71], which are the two singlet (symmetric and completely antisymmetric) and doublet subspace. This allows us to reduce the dimensionality $\mathcal{N} \propto N^{2}$ (see Eq. (2.38)) of the Hilbert-space by restricting the calculations to one of the subspaces.

We choose the symmetric one which leads to a reduction by a factor of approximately six. The origin of this number becomes clear if one realizes that the eigenvalues of the uncoupled homogeneous trimer are most likely six-fold degenerate which occurs if $n_{1} \neq n_{2} \neq n_{3} \neq n_{1}$. If two boson numbers agree (e.g. $n_{1}=n_{2} \neq n_{3}$ ) the corresponding degeneracy is threefold while the state of equal occupation (exists only if $\bmod (N, 3)=0) n_{1}=n_{2}=n_{3}$ is not degenerate. Following Ref. [71] we define the orthonormal basis

$$
\left\{\left|n_{1}, n_{2}, n_{3}\right\rangle_{S}\right\}_{n_{1} \leq n_{2} \leq n_{3}, n_{1}+n_{2}+n_{3}=N}
$$

through the basis states ( the subscript $S$ indicates the symmetric sub-space)

$$
\left|n_{1}, n_{2}, n_{3}\right\rangle_{S}=\frac{1}{\alpha_{S}} \sum_{P}\left|P\left(n_{1}\right), P\left(n_{2}\right), P\left(n_{3}\right)\right\rangle
$$

where $P$ runs over the permutations of $\left(n_{1}, n_{2}, n_{3}\right)$ and

$$
\begin{array}{lll}
\alpha_{S}\left(n_{1}, n_{2}, n_{3}\right)=\sqrt{6} & \text { if } & n_{1} \neq n_{2} \neq n_{3} \\
\alpha_{S}\left(n_{1}, n_{2}, n_{3}\right)=\sqrt{12} & \text { if } & n_{1}=n_{2} \neq n_{3} \text { or } n_{1} \neq n_{2}=n_{3} \text { or } n_{1}=n_{3} \neq n_{2} \\
\alpha_{S}\left(n_{1}, n_{2}, n_{3}\right)=6 & \text { if } & n_{1}=n_{2}=n_{3}
\end{array}
$$

The main motivation to use the symmetric subspace is of a computational nature as it allows us to consider systems of up to several hundred bosons. Of course, we have checked that this procedure does not affect the results. Indeed, when analyzing the spectral statistics presented in Section 3.1 it is indispensable to perform the analysis for the individual subspace in order to get a meaningful result and eliminate inherent degeneracies (see also Appendix A).

\subsubsection{Classical dynamics}

Upon taking the semiclassical limit of the $\mathrm{BHH}$, the system of bosons on the lattice is described by $f$ nonlinear oscillators that are linearly coupled. As in the quantum case the classical Hamiltonian $(2.23,2.27)$ has $f$ degrees of freedom and two constants of motion, one of them being the energy $\tilde{E}=\tilde{\mathcal{H}}$. The quantum mechanical conservation of the particle number $N$ translates into conservation of total action $I=\sum_{i} I_{i}$ or respectively total oscillator strength $\sum\left|A_{i}\right|^{2}$. 

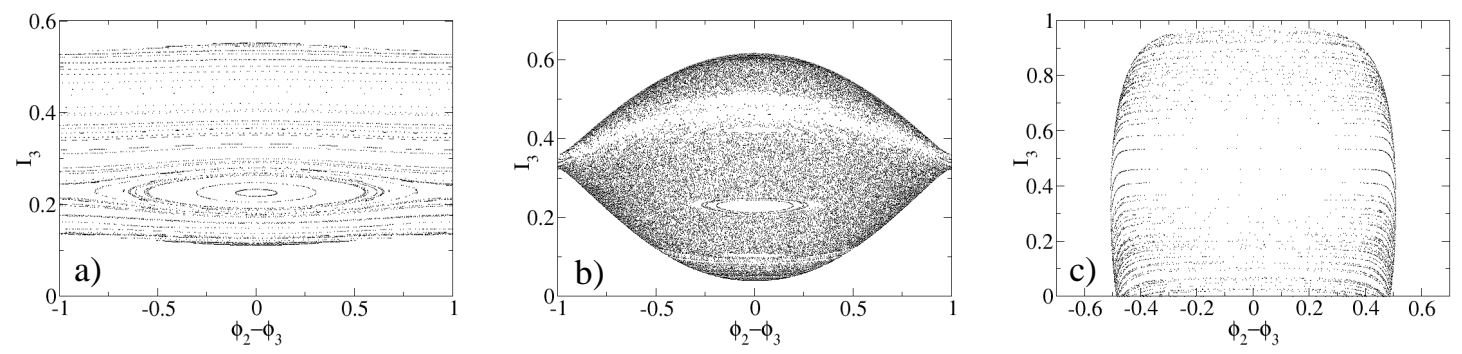

Figure 2.5.: Poincaré sections of the phase space belonging to the classical trimer for $N=1, k=1$ with energy $\tilde{E}=0.2$ and different parameter values a) $\lambda=0.005$, b) $\lambda=0.05$, c) $\lambda=2$. On the $y$-axis we plot the action $I_{3}$ while on the $x$-axis the difference $\varphi_{2}-\varphi_{3}$ (in units of $\pi$ ) is plotted. This figure was created with a program provided by Gim Seng $\mathrm{Ng}$ [156].

The classical dynamics depends both on the scaled energy $\tilde{E}=E / \tilde{U} N$ and the dimensionless parameter $\lambda=k / \tilde{U}[188,203,71,92,155]$. For $\lambda \rightarrow 0$ the interaction term dominates and the system behaves as a set of uncoupled sites. This limit is also known as the localmode picture [21] while the opposite case, i.e. $\lambda \rightarrow \infty$, is called the normal-mode picture $[188,76,215]$ where the kinetic term is the dominant one. In both limits the motion is integrable while for intermediate values of $\lambda$ and a number of sites $f>2$ the it has a chaotic component [64].

We will investigate the classical dynamics in more detail at the beginning of the next chapter, as well as in Section 4.4, where we also compare it to quantum dynamics, and Section 5.4. In Fig. 2.5 we give some examples of the classcal dynamics (2.25) of the trimer, by plotting Poincaré sections of the phase space for constant energy $\tilde{E}$ and various values of the parameter $\lambda$. 


\section{Parametric Bose-Hubbard Hamiltonians: Spectra and Eigenfunctions}

Consider a system whose Hamiltonian $\mathcal{H}(I, \varphi ; X)$ depends not only on a set of canonical variables $(I, \varphi)$ but also on a parameter $X$ which in principle might be time-dependent, i.e. $X=X(t)$. Such parametrically driven Hamiltonians [212] frequently appear in various fields like mesoscopic electronic, atomic and molecular physics.

One of the paradigms of parameter dependent Hamiltonians is the so-called piston model [52]. Here $X(t)$ denotes the position of a large object (the piston) that is located within an enclosure and is only weakly perturbed by the remainder of the system, e.g. a gas particle, described by the variables $(I, \varphi)$, moving inside the enclosure. In molecular physics, for example, $X(t)$ is associated with the motion of the nuclei in a molecule and the electronic degrees of freedom represent the weak perturbation. As $X(t)$ is changing (for a fixed configuration of the nuclei) the electronic system undergoes radiationless transitions [140]. In mesoscopic physics, the piston model has recently been realized using electrons moving in a quantum dot where $X(t)$ parameterizes the dot's shape, which is determined by the applied gate voltages. By (periodically) changing the shape of the dot one can study for example the dissipation of energy into the system.

Yet a different application of parametric Hamiltonians is associated with a charged particle moving inside a ring (Aharonov-Bohm topology). Here, the parameter $X(t)$ represents a perpendicular magnetic flux that is concentrated the ring's hole. According to Faraday's law, the time-derivative of the flux $\dot{X}(t)=V$ is the electromotive force induced in the loop leading to a current $I$ that flows inside the ring. Inserting Ohm's law $V=R I$ (where $R$ is the resistance) into Joule's law $d E / d t=V I=1 / R V^{2}$ we find that the conductance $G=1 / R$ is proportional to the dissipated energy. Thus, in such a setup $G$ can be measured without the need to open the system.

Though the above examples are mainly treated using a single-particle picture, it is apparent that the concept of parametric Hamiltonians is fundamental in physics, including many-body systems. In this respect, (ultra-)cold atoms loaded in optical lattices [125] provide an excellent framework to study driven interacting systems and compare the theoretical predictions to experimental results, identify limitations and propose new theoretical methods.

In our analysis of the Bose-Hubbard Hamiltonian (BHH), we choose as a control parameter the tunneling strength $X=k$ between neighboring lattice sites (see Chapter 2). We are interested in the evolution of the boson configuration as $k_{0} \rightarrow k_{0}+\delta k$ is being varied. Naturally, the first step in the study of the parametric BHH is the case where the perturbation $\delta k$ is not time-dependent but constant. Therefore, the topic of the present chapter comprises 
the static properties like spectral statistics, the characteristics of the applied perturbation, and the parametric evolution of eigenfunctions [115] which will lead the way to study the quantum dynamics in the coming chapter.

Throughout this work we always assume that the perturbed Hamiltonian $\mathcal{H}(k)$ as well as the unperturbed Hamiltonian $\mathcal{H}\left(k_{0}\right)$ generate classical dynamics of the same nature, i.e., that the perturbation $\delta k=k-k_{0}$ is classically small, $\delta k<\delta k_{\mathrm{cl}}$. This assures the applicability of classical linear response theory (LRT). Note, however, that this assumption is not sufficient to guarantee the validity of quantum mechanical linear response theory. Our aim is to identify the limitations of quantum mechanical LRT, and the applicability of semiclassical methods. At the same time, we address the implications of classically chaotic dynamics, and the route to quantum-classical correspondence.

This chapter is structured as follows: In the next section, we analyze the spectral properties of the Bose-Hubbard Hamiltonian. We then establish a semiclassical connection between the power spectrum and the quantum mechanical bandprofile of the perturbation operator and study its statistical properties. In Section 3.2, we revise the banded random matrix modeling developed by Wigner and motivate an improved random matrix theory (IRMT) model which takes the structured bandprofile into account [112]. After that we introduce the concept of parametric regimes and demonstrate its applicability in the analysis of the parametric evolution of eigenstates [59]. In Section 3.6, we present our findings [115] for the $\mathrm{BHH}$ and compare them with the predictions from the Wigner RMT model. We reveal the importance of the structured bandprofile of the perturbation operator and show how it can be included in an infinite order perturbation theory. In the regime of strong perturbations $\delta k$, where perturbation theory fails, we apply semiclassical considerations and show that RMT modeling leads to a strong non-perturbative response effect that differs from the semiclassical behavior.

\subsection{Statistical properties of the BHH: Spectra and bandprofile}

In a substantial part of the existing literature (see, for example, Ref. [94]), the BoseHubbard Hamiltonian (2.10) was investigated using a mean-field (classical) picture. In contrast, quantum mechanical calculations of a BHH are often limited by severe computational memory restrictions. However, it is possible to treat lattices consisting of a few sites. In this respect the $\mathrm{BHH}$ trimer (see Section 2.6) is especially interesting since the underlying classical dynamics can be chaotic, thus paving the way to understand larger lattices. ${ }^{1}$ The trimer has been studied quite extensively in the semiclassical regime [77, 110, 92, 46]. Surprisingly enough, the quantum trimer [71, 155] (not to mention larger lattices [136]) is barely treated. As a matter of fact, the majority of the quantum studies are focused on the statistical properties of levels $[71,64,49]$ or are limited to the ground state properties of the

${ }^{1}$ See also Subsection 2.6.1. 

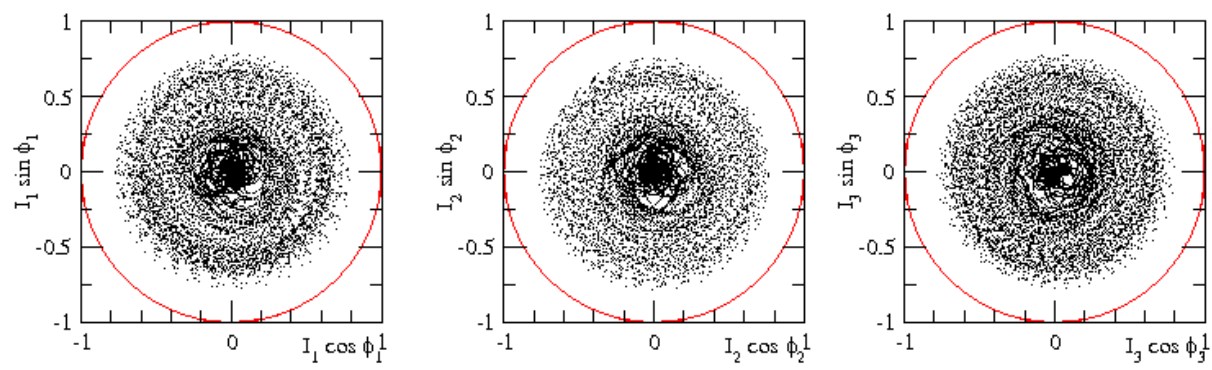

Figure 3.1.: Classical time evolution of $N=230$ particles (bosons) in the trimeric BHH for $\tilde{U}=280$ and energy $\tilde{E}=0.26$ according to Eq. (2.25). The red line indicates the accessible phase space if all particles were located on the corresponding site.

BHH $[143,18,120]$. Substantially more insight to this complex quantum system is gained through the analysis of the eigenstates [115].

In this chapter we study the trimeric BHH model (see Section 2.6) as a control parameter, the coupling strength between lattice sites: $k_{0} \rightarrow k_{0}+\delta k$ is changed. In our analysis, we therefore consider

$$
\hat{H}=\hat{H}_{0}-\delta k \hat{B}
$$

where the coupling operator is

$$
\hat{B}=\sum_{\langle i, j\rangle} \hat{b}_{i}^{\dagger} \hat{b}_{j}
$$

and the unperturbed Hamiltonian $\hat{H}_{0}$ is given by Eq. (2.39) with $k=k_{0}$. Quantum mechanically, we work in the $\hat{H}_{0}$ eigenbasis. In this basis $\hat{H}_{0}$ becomes diagonal, i.e., $\mathbf{E}_{0}=E_{m}^{(0)} \delta_{m n}$ where $\left\{E_{m}^{(0)}\right\}$ are the ordered eigenvalues and we can write

$$
\mathbf{H}=\mathbf{E}_{0}-\delta k \mathbf{B}
$$

A fixed assumption of this work is that the perturbation is classically small $\delta k \ll \delta k_{\mathrm{cl}}$, i.e., the corresponding classical Hamiltonians $\mathcal{H}_{0}$ and $\mathcal{H}$ generate dynamics of the same nature. Below we will concentrate on parameter values, where the classical dynamics is predominantly chaotic.

As a preliminary step, we determine the regime of classically chaotic motion. Following Refs. $[76,188,70]$ the analysis is based on the nature of the phase space and the power spectrum $\tilde{C}(\omega)$ of the perturbation operator $\mathcal{B}$ which we will discuss in detail in Subsection 3.1.2. While regular motion results in isolated peaks in $\tilde{C}(\omega)$, a continuous (but possibly structured) power spectrum indicates chaoticity. Accordingly, the classically smallness condition $\delta k \ll \delta k_{\mathrm{cl}}$ can be operatively defined as the perturbation strength that leaves $\tilde{C}(\omega)$ unaffected. We have found that for $0.04<\lambda=k / \tilde{U}<0.2$ and in an energy interval $\tilde{H} \approx 0.26 \pm 0.02$ the motion is predominantly chaotic. In the simulations below we will use $k_{0} \approx 15$, an effective interaction strength $\tilde{U}=U N \approx 280$ and the number of particles 


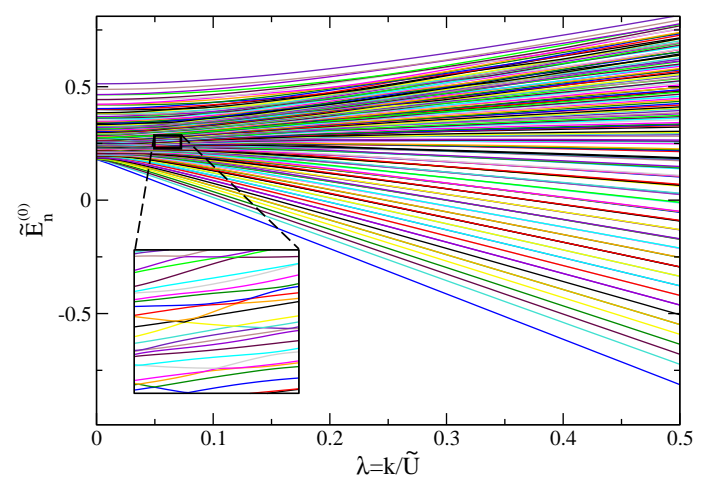

Figure 3.2.: Parametric evolution of the eigenvalues $\tilde{E}_{n}^{(0)}$ as a function of the parameter $\lambda$. The number of bosons is $N=40$ and the effective interaction strength is $\tilde{U}=280$. In the main figure the entire spectrum is plotted while the inset is a magnification of the small box. One observes a qualitative change in the spectrum as $\lambda$ is changed. See text for details.

$N=230$ if not stated otherwise. In this regime, we get $\delta k_{\mathrm{cl}}=20$. In Fig. 3.1 we report some representative trajectories in phase-space that illustrate the ergodic nature of the classical motion (see also the Poincaré sections in Fig. 2.5).

It is then natural to ask how the classically chaotic behavior is reflected upon quantization. As we shall see in the following subsections, chaos manifests itself mainly in two quantities; the spectral statistics and the averaged profile $\left\langle\left|\mathbf{B}_{m n}\right|^{2}\right\rangle$ of the perturbation operator.

\subsubsection{Energy levels}

We start with the discussion of the spectral statistics of the quantum BHH trimer. In Fig. 3.2 we plot the parametric evolution of the eigenvalues $\tilde{E}_{n}^{(0)}$ as a function of $\lambda$ for fixed effective interaction strength $\tilde{U}=280$. From Fig. 3.2 one observes that the spectrum becomes rather regular for very large $\lambda$. Indeed, for $\lambda \rightarrow \infty$ a transformation to the local modes of the system diagonalizes the Hamiltonian and yields equidistant eigenvalues. In the local-mode limit, i.e. $\lambda \rightarrow 0$, the eigenvalues of $\hat{H}_{0}$ are obtained immediately from (2.39) and are partly degenerate. ${ }^{2}$ However, in an intermediate $\lambda$-regime one observes a different behavior: The evolution is irregular and the levels seem to repel each other (see inset). This is a manifestation of the classical chaotic behavior [106, 197].

In order to establish this statement we turn to the statistical properties of the spectra. In particular, we will study the level spacing distribution $P(S)$ (see Appendix A) where

$$
S_{n}=\frac{E_{n+1}-E_{n}}{\Delta(E)}
$$

are the level spacings which are unfolded with respect to the local mean level spacing $\Delta(E)$. The level spacing statistics have already been investigated for the chaotic BHH [71, 64, 49, $136]$ and represent one of the most popular measures used in quantum chaos studies [197, 106]. In this context, it turns out that the sub- $\hbar$ statistical features of the energy spectrum are

\footnotetext{
${ }^{2}$ We note that these are 'accidental' degeneracies. In contrast, systematic degeneracies due to the symmetry of the model are eliminated by restricting the calculation to the symmetric subspace. See also Ref.[71].
} 


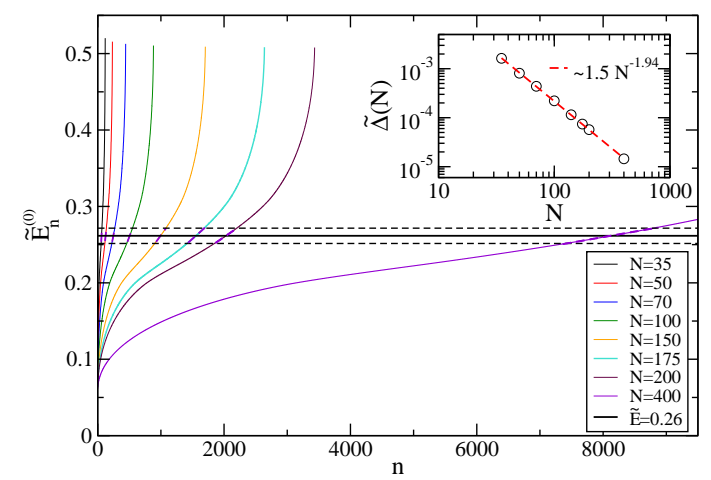

Figure 3.3.: Main figure: Energy spectra $\tilde{E}_{n}^{(0)}$ of the BHH trimer for various numbers of bosons $N$ and $\tilde{U}=280$ plotted against the level index $n$. The dashed horizontal lines indicate the energy range in which the further analysis is performed. The inset shows the local mean level spacing $\tilde{\Delta}=\Delta / \tilde{U} N$ (o) in that energy range as a function of $N$. The red dashed line is a fit to the data points and has negative slope 1.94 .

"universal", and obey the predictions of Wigner's random matrix theory (RMT) [208, 209] which we will introduce later in Section 3.2. In contrast, non-universal, i.e. system specific, features are reflected only in the large scale properties of the spectrum and constitute the fingerprints of the underlying classical dynamics.

We determine the scaling of the mean level spacing $\Delta$ by using the fact that the Hilbertspace dimension $\mathcal{N}$ grows as $\mathcal{N} \propto N^{2}$ (see Subsection 2.6.2) while the energy $\Delta E$ spanned by the system is proportional to $\tilde{U} N \times \tilde{E}$, where $\tilde{E}$ is constant (see Eqs. (2.23) and (2.19)). Then the global scaling of the mean level spacing should be ${ }^{3}$

$$
\Delta \sim \frac{\Delta E}{\mathcal{N}} \sim \frac{\tilde{U}}{N}
$$

In Fig. 3.3 we report spectra for $\tilde{U}=280$ and various $N$. The numerical data are in good agreement with the theoretical expectation (3.5). We use the spectral data to estimate the proportionality factor at the energy window around $\tilde{E}=0.26$ where most of our calculations are done and obtain

$$
\Delta \approx 1.5 \frac{\tilde{U}}{N}
$$

Next, we calculate $P(S)$. For chaotic systems the latter follows the so-called Wigner surmise (see Appendix A)

$$
P_{\text {chaotic }}(S)=\frac{\pi}{2} S e^{-\frac{\pi}{4} S^{2}},
$$

indicating that there is a linear repulsion between nearby levels. Instead, for generic integrable systems there is no correlation between the eigenvalues and the distribution $P(S)$ is Poissonian

$$
P_{\text {integrable }}(S)=e^{-S} \text {. }
$$

In order to quantify the degree of chaoticity, various phenomenological formulas have been suggested that interpolate between these two limiting cases like the Berry-Robnik [28] or the Brody distribution [36]. We focus on the latter which is given by

$$
P_{q}(S)=\alpha S^{q} e^{-\beta S^{1+q}},
$$

\footnotetext{
${ }^{3}$ We stress once again, that $\tilde{U}$ has to be kept constant while increasing $N$. See around Eq. (2.21).
} 

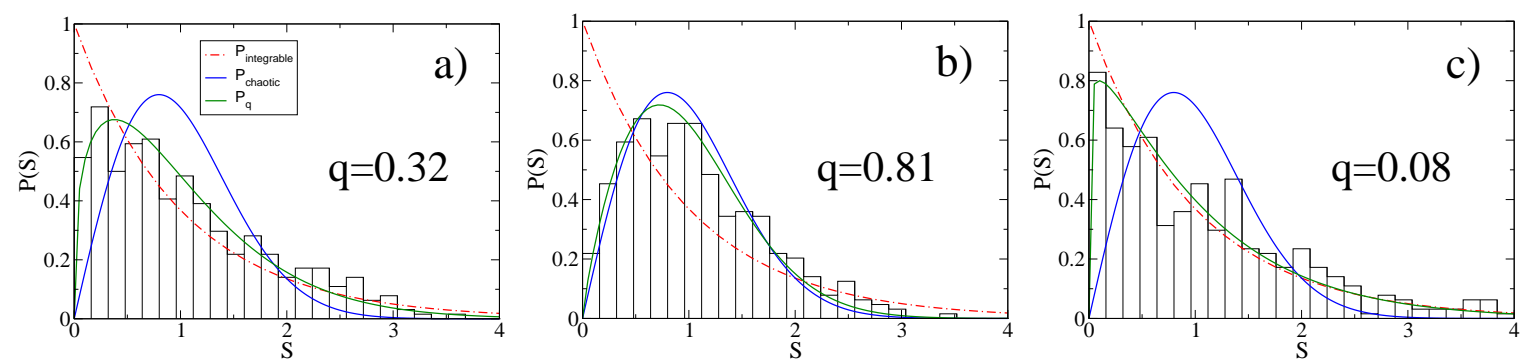

Figure 3.4.: The level spacing distribution $P(S)$ of the BHH trimer for three representative values of the dimensionless ratio $\lambda=k / \tilde{U}$ which controls the underlying classical dynamics: a) $\lambda=0.025(k=7)$, b) $\lambda=0.05(k=14.5)$, and c) $\lambda=0.35(k=100)$. The red dash-dotted line corresponds to the Poissonian distribution (3.8) which is expected for integrable systems, the solid blue line corresponds to the Wigner surmise (3.7) (chaotic systems) while the solid green line represents the fitted Brody distribution (3.9). In Figure 3.5 we report the fitted Brody parameter $q$ for various values of $\lambda$. The System corresponds to $N=230$ bosons and $\tilde{U}=280$. The histograms include the 400 relevant levels around $\tilde{E}=0.26$.

where $\alpha=(1+q) \beta, \beta=\Gamma^{1+q}[(2+q) /(1+q)]$ and $\Gamma$ is the Gamma function. The two parameters $\alpha, \beta$ are determined by the condition that the distribution is normalized with a mean equal to one [37]. The so-called Brody-parameter $q$ is then obtained from fitting the $P_{q}(S)$ to the numerically evaluated level spacing distribution. One readily verifies that for $q=0$, the distribution $P_{q}(S)$ is Poissonian while for $q=1$ it takes the form of (3.7). In Fig. 3.4 we report the distribution $P(S)$ of the levels in the relevant energy window around $\tilde{E}=0.26$ together with the fitted $P_{q}$ for some representative cases of $\lambda$. $^{4}$ One observes a qualitative change in $P(S)$ as $\lambda$ is increased from zero. This is in agreement with the parametric plot of the levels (see Fig. 3.2).

The quantitative analysis is presented in Fig. 3.5 where we plot $q$ against $\lambda$ which governs the underlying classical dynamics. For very small and very large $\lambda$ the Brody parameter is small indicating classically regular motion while for intermediate values we find $q \sim 1$ corresponding to classically chaotic motion. The observed range of large $q$ is in excellent agreement with the classical analysis that led to a range of $0.04<\lambda<0.2$ for the motion to be predominantly chaotic in the discussed energy window.

\subsubsection{The bandprofile}

Consider a given ergodic trajectory $(I(\tilde{t}), \varphi(\tilde{t}))(\tilde{t}=\tilde{U} t$ is the rescaled time) on the energy surface $\tilde{\mathcal{H}}\left(I(0), \varphi(0) ; k_{0}\right)=\tilde{E}$ like the one shown in Fig. 3.1. We can associate with it a

\footnotetext{
${ }^{4}$ We note that for level spacing distribution it is essential [197] to distinguish levels from different symmetry classes. Here, the statistics is performed over the symmetric singlet states of the BHH. See also Ref.[71] and Appendix A.
} 


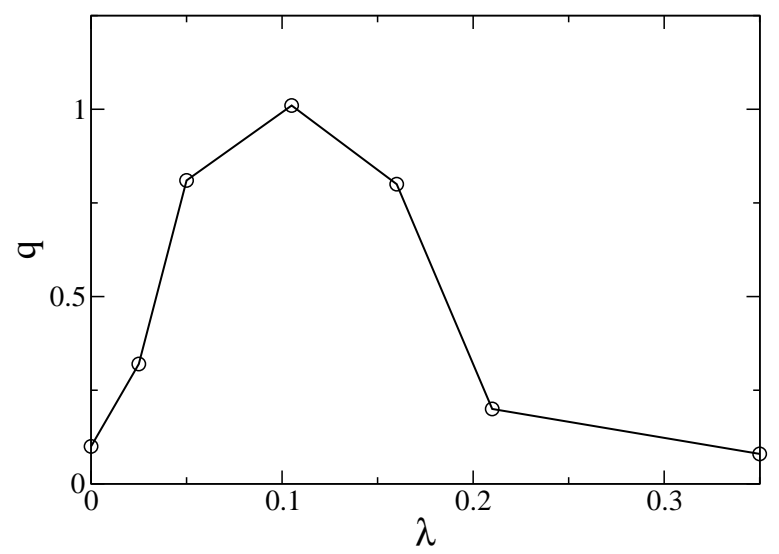

Figure 3.5.: The Brody parameter $q$ for the BHH plotted against the dimensionless ratio $\lambda$ which controls the underlying classical dynamics. The values of $q$ are obtained from fits to $P_{q}(S)$ around $\tilde{E}=0.26$ as reported in Fig. 3.4. The System corresponds to $N=230$ bosons and $\tilde{U}=280$. See text for details.

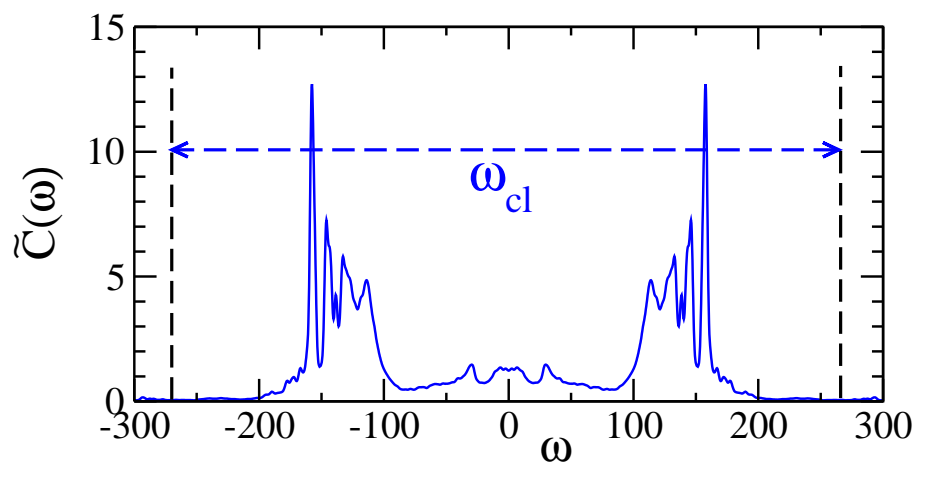

Figure 3.6.: The power-spectrum of the classical BHH trimer (2.23) (with $f=3, v_{i}=0$ ) at energy $\tilde{E}=0.26, \tilde{U}=280$, and $\lambda_{0}=0.053$. The classical cut-off frequency $\omega_{\mathrm{cl}}=\tilde{\omega}_{\mathrm{cl}} \tilde{U} \approx 280$ is indicated by vertical dashed lines.

stochastic-like variable

$$
\tilde{\mathcal{F}}(\tilde{t})=-\frac{\partial \tilde{\mathcal{H}}}{\partial k}(I(\tilde{t}), \varphi(\tilde{t}) ; k(\tilde{t}))
$$

which can be seen as a generalized force. For the BHH (3.3) this is simply the perturbation term $\tilde{\mathcal{F}}=\sum_{i \neq j} \sqrt{I_{i} I_{j}} \exp ^{i\left(\varphi_{j}-\varphi_{i}\right)}$ which corresponds to a momentum boost since it changes the kinetic energy [165]. It may have a non-zero average, i.e. a "conservative" part, but below we are interested only in its fluctuations.

In order to characterize the fluctuations of $\tilde{\mathcal{F}}(\tilde{t})$ we introduce the autocorrelation function

$$
C(\tilde{\tau})=\langle\tilde{\mathcal{F}}(\tilde{t}) \tilde{\mathcal{F}}(\tilde{t}+\tilde{\tau})\rangle-\left\langle\tilde{\mathcal{F}}^{2}\right\rangle
$$

where $\tilde{\tau}=\tilde{U} \tau$ is a rescaled time. The angular brackets denote an averaging which is either 
micro-canonical over some initial conditions $(I(0), \varphi(0))$ or temporal due to the assumed ergodicity.

For generic chaotic systems (described by smooth Hamiltonians), the fluctuations are characterized by a short correlation time $\tilde{\tau}_{\mathrm{cl}}$, after which the correlations are negligible. In generic circumstances $\tilde{\tau}_{\mathrm{cl}}$ is essentially the ergodic time. For our model system we have found $\tilde{\tau}_{\mathrm{cl}} \sim 2 \pi$.

The power spectrum of the fluctuations $\tilde{C}(\tilde{\omega})$ is defined by a Fourier transform:

$$
\tilde{C}(\tilde{\omega})=\int_{-\infty}^{\infty} C(\tilde{\tau}) e^{i \tilde{\omega} \tilde{\tau}} \mathrm{d} \tilde{\tau} .
$$

This power spectrum is characterized by a cut-off frequency $\omega_{\mathrm{cl}}$ which is inverse proportional to the classical correlation time

$$
\tilde{\omega}_{\mathrm{cl}}=\frac{2 \pi}{\tilde{\tau}_{\mathrm{cl}}}
$$

The classical power spectrum for the BHH trimer is shown in Fig. 3.6. Indeed, we see that $\tilde{C}(\omega)$ has a frequency support which is bounded by $\tilde{\omega}_{\mathrm{cl}} \approx 1$ corresponding to $\omega_{\mathrm{cl}} \approx 280$ (indicated by dashed vertical lines in Fig. 3.6). Having a bounded (continuous) support in $\tilde{C}(\omega)$ means that the perturbation does not excite all modes of the system but only those within a finite range..$^{5}$ These characteristics of the power spectrum are universal for generic chaotic systems. Finally, we see that within the frequency support the power spectrum $\tilde{C}(\tilde{\omega})$ is structured, reflecting system-specific properties of the underlying classical dynamics.

As we explain in detail in Appendix A.2, the classical power spectrum $\tilde{C}(\tilde{\omega})$ is associated with the quantum mechanical perturbation matrix $\mathbf{B}$ according to the following semiclassical relation $[85,171]$

$$
\left\langle\left|\mathbf{B}_{n m}\right|^{2}\right\rangle=\frac{N^{2} \Delta}{\tilde{U} 2 \pi} \tilde{C}\left(\omega=\frac{E_{n}-E_{m}}{\hbar}\right) .
$$

Hence the matrix elements of the perturbation matrix $\mathbf{B}$ are extremely small outside of a band of width

$$
\Delta_{b}=\hbar \omega_{\mathrm{cl}} \text {. }
$$

It is common to define also a dimensionless bandwidth $b=\Delta_{b} / \Delta$. In the inset of Fig. 3.7 we show a snapshot of the perturbation matrix $\left|\mathbf{B}_{n m}\right|^{2}$ which clearly exhibits a band-structure. In the same figure we also display the scaled quantum bandprofile for $N=230$. The agreement with the classical power spectrum $\tilde{C}(\omega)$ is very good. We note that the relation (3.14) is very robust $[59,112,115]$ and holds even for small boson numbers $N \approx 50$. Combining Eqs. (3.6), (3.15) with $\tilde{\omega}_{\mathrm{cl}} \approx 1$ (see above) and the definition of $b$ we find for the chaotic regime around $\tilde{E}=0.26$ that $b \sim 0.6 N$ which is confirmed by the numerics.

At the beginning of the section we were asking "Where does the classical chaos appear in the quantum mechanics?" Up to here we can give the following answer: Classical chaos

\footnotetext{
${ }^{5}$ This behavior would be expected for a hard chaotic system where the correlation time $\tilde{\tau}_{\mathrm{cl}}$ vanishes.
} 


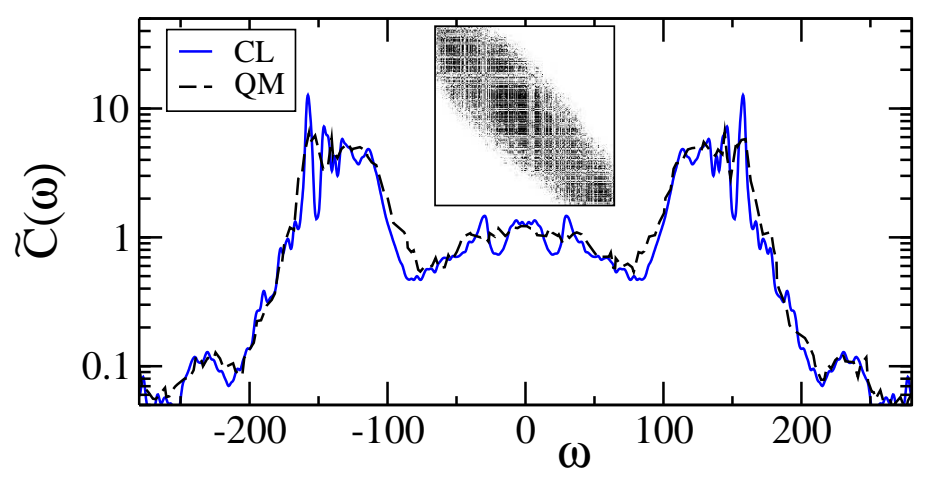

Figure 3.7.: The bandprofile $\left(2 \pi \tilde{U} / N^{2} \Delta\right) \cdot\left|\mathbf{B}_{n m}\right|^{2}$ versus $\omega=\left(E_{n}-E_{m}\right) / \hbar$ is compared with the classical power spectrum $\tilde{C}(\omega)$. The number of particles is $N=230$ and $\lambda_{0}=0.053$. Inset: snapshot of the perturbation matrix $\mathbf{B}$.

manifests itself in the spectral statistics and in the appearance of a bandprofile of the perturbation matrix $\mathbf{B}[59,112,115]$. It is important to realize that through this type of quantum classical correspondence (QCC), chaos introduces an additional energy scale in the quantum system. Hence we end up with two distinct energy scales. One is obviously the mean level spacing (see above)

$$
\Delta \propto \tilde{U} / N,
$$

while the other one is the bandwidth

$$
\Delta_{b}=b \Delta \propto \tilde{U} .
$$

The latter energy scale is also known in the corresponding literature as the "non-universal" energy scale [26], or in the case of diffusive motion, as the Thouless energy [118]. ${ }^{6}$ One has to notice that deep in the semiclassical limit $\hbar \rightarrow 0(N \rightarrow \infty)$ these two energy scales differ enormously from one another. We shall see in the following sections and chapters that this scale separation has dramatic consequences on the theory of driven quantum systems.

\subsubsection{Distribution of couplings}

We further investigate the statistical properties of the matrix elements $\mathbf{B}_{n m}$ of the perturbation matrix by studying their distribution. RMT assumes that upon appropriate "unfolding" they must be distributed in a Gaussian manner. The unfolding aims to remove system specific properties and to reveal the underlying universality. It is carried out by normalizing the matrix elements with the local standard deviation $\sigma=\sqrt{\left\langle\left|\mathbf{B}_{n m}\right|^{2}\right\rangle}$ related through Eq. (3.14) with the classical power spectrum $\tilde{C}(\omega)$.

The existing literature is not conclusive about the distribution of the normalized matrix elements $w=\mathbf{B}_{n m} / \sigma$. Specifically, Berry [24] and more recently Prosen [171, 169], claimed

\footnotetext{
${ }^{6}$ The dimensionless parameter $b$ scales like $b \propto \hbar^{-(d-1)}$ and in the frame of mesoscopic systems is recognized as the dimensionless Thouless conductance [118].
} 


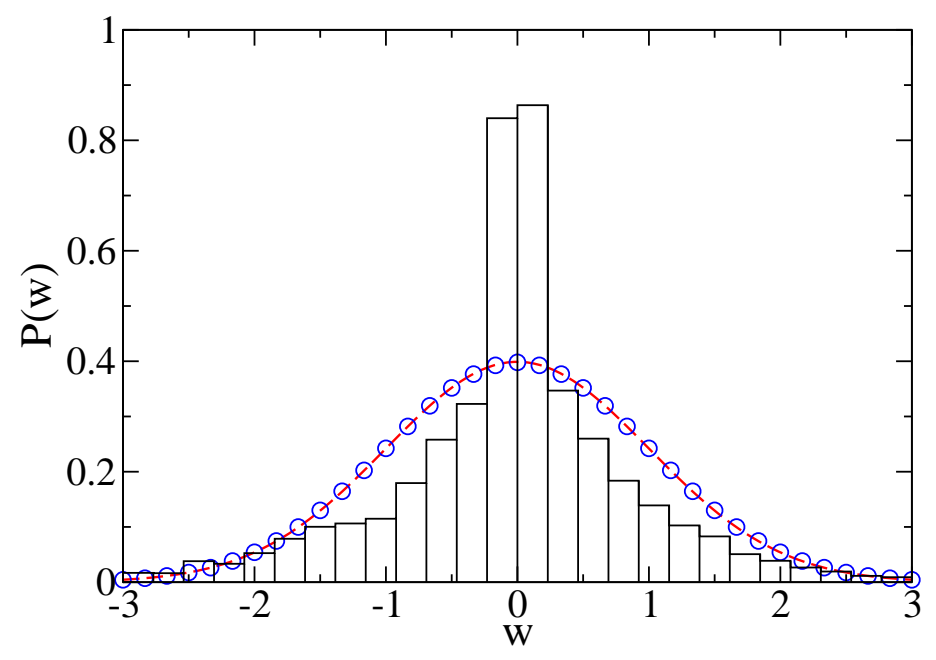

Figure 3.8.: Distribution of rescaled matrix elements $w$ around $\tilde{E}=0.26$ rescaled with the averaged bandprofile. The dashed red line corresponds to the standard normal distribution while the circles (o) correspond to a best fit from Eq. (3.18) with a fitting parameter $N=342$. The system corresponds $N=230, \tilde{U}=280$.

that $\mathcal{P}(w)$ should be Gaussian. On the other hand, Austin and Wilkinson [14] have found that the Gaussian is approached only in the limit of high quantum numbers while for small numbers, i.e., low energies, a different distribution applies, namely

$$
P_{\text {couplings }}(w)=\frac{\Gamma\left(\frac{N}{2}\right)}{\sqrt{\pi N} \Gamma\left(\frac{N-1}{2}\right)}\left(1-\frac{w^{2}}{N}\right)^{(N-3) / 2} .
$$

This is the distribution of the elements of an $N$-dimensional vector, distributed randomly over the surface of an $N$-dimensional sphere of radius $\sqrt{N}$. For $N \rightarrow \infty$ this distribution approaches a Gaussian.

In Fig. 3.8 we report the distribution $\mathcal{P}(w)$ for the perturbation matrix $\mathbf{B}$. The dashed line corresponds to a Gaussian of unit variance while the circles are obtained by fitting Eq. (3.18) to the numerical data using $N$ as a fitting parameter. In this case the fit yielded $N=342$ and accordingly the latter two curves are virtually identical. The large $N$ value implies that the system should be located already deep in the semiclassical regime and is in agreement with the correspondence with the findings of the previous section. Interestingly, also for smaller system sizes the obtained parameter $N$ is quite high. We observe that the Gaussian fairly resembles our numerical data but there are deviations especially for matrix elements close to zero. We attribute these deviations to the existence of small stability islands in the phase space. Trajectories started in those islands cannot reach the chaotic sea and vice versa. Quantum mechanically, the consequence of this would be vanishing matrix elements $\mathbf{B}_{n m}$ which represent the classically forbidden transitions. 
$\mathrm{H}=\left[\begin{array}{cc}1 \Delta & 0 \\ 2 \Delta & \\ 3 \Delta & \\ 0 & \ddots\end{array}\right]+\delta \mathrm{k}\left[\begin{array}{l}\mathbf{b} \\ 0\end{array}\right]$

Figure 3.9.: The WBRM-model with constant level spacing $\Delta$ and bandwidth $b$.

\subsection{RMT modeling}

More than 50 years ago, E. P. Wigner [208, 209] proposed a simplified model with a Hamiltonian given by Eq. (3.3) and $\mathbf{B}$ being a banded random matrix [84, 83, 96]. It is known as the Wigner banded random matrix model (WBRM). This approach is attractive both analytically and numerically. Analytical calculations are greatly simplified by the assumption that the off-diagonal terms can be treated as independent random numbers. Also from a numerical point of view it is quite a tough task to calculate the true matrix elements of B. It requires a preliminary step where the chaotic $\hat{H}_{0}$ is diagonalized and limited memory restricts the matrix size. For the Bose-Hubbard Hamiltonian we were able to handle matrices of final size $\mathcal{N}=30,000$ maximum. This should be contrasted with the WBRM simulations, where using self-expanding algorithm [121, 57, 112] we were able to handle system sizes up to $\mathcal{N}=1,000,000$ along with significantly reduced CPU time. We would like to stress, however, that the underlying assumption of the WBRM, namely that the offdiagonal elements are uncorrelated random numbers, has to be treated with extreme care. The applicability of this model is therefore a matter of conjecture which we will test in the following.

In fact, the WBRM model involves additional simplifications, namely that the diagonal matrix $\mathbf{E}_{0}$ has elements which are the ordered energies $\left\{E_{n}\right\}$, with mean level spacing $\Delta^{7}$ and the perturbation matrix $\mathbf{B}$ has a rectangular bandprofile of bandwidth $b$. Within the band $0<|n-m| \leq b$ the elements are independent random variables given by a Gaussian distribution with zero mean and a variance $\sigma^{2}=\left\langle\left|\mathbf{B}_{n m}\right|^{2}\right\rangle$ while outside the band they vanish (see Fig. 3.9 for an illustration).

Given the bandprofile, we can use Eq.(3.14) in reverse direction to calculate the correlation function $C(\tau)$. For the WBRM model we get

$$
\begin{aligned}
C(\tau) & =\int_{-\infty}^{\infty} \mathrm{d} E_{k} g\left(E_{k}\right)\left|\mathbf{B}_{n m}\right|^{2} e^{-i E_{k} \tau / \hbar}=\frac{\sigma^{2}}{\Delta} \int_{-\Delta_{b}}^{\Delta_{b}} \mathrm{~d} E_{k} e^{-i E_{k} \tau / \hbar} \\
& =2 \sigma^{2} b \operatorname{sinc}\left(\frac{\tau}{\tau_{\mathrm{cl}}}\right)
\end{aligned}
$$

where $\tau_{\mathrm{cl}}=\hbar / \Delta_{b}$. Thus, there are three parameters $(\Delta, b, \sigma)$ that define the WBRM model.

\footnotetext{
${ }^{7}$ This equal spaced diagonal matrix is also known as a "picket-fence" Hamiltonian.
} 
While the WBRM model assumes that $\mathbf{B}$ has a rectangular bandprofile, a simple inspection of Fig. 3.7 shows that this is not the case for our BHH model (2.39). We eliminate this simplification by introducing a RMT model that is even closer to the dynamical one. To this end, we randomize the signs of the off-diagonal elements of the perturbation matrix $\mathbf{B}$ keeping its band-structure intact. This procedure leads to a random model that exhibits only universal properties but lacks any semiclassical limit. We will refer to it as the improved banded random matrix model (IRMT).

\subsection{Parametric evolution of eigenfunctions}

As we change the parameter $\delta k$ in the Hamiltonian (3.3), the instantaneous eigenstates $\{|n(k)\rangle\}$ evolve and undergo structural changes. In order to finally understand the actual quantum dynamics, where $k=k(t)$ is time-dependent, it is important to understand these structural changes. This leads to the introduction of the "kernel"

$$
P(n \mid m)=\left|\left\langle n\left(k_{0}+\delta k\right) \mid m\left(k_{0}\right)\right\rangle\right|^{2},
$$

which can be interpreted in two ways as we schematically depict in Fig. 3.10. If regarded as a function of $m, P(n \mid m)$ represents the overlap of a given perturbed eigenstate $\left|n\left(k_{0}+\delta k\right)\right\rangle$ with the eigenstates $\left|m\left(k_{0}\right)\right\rangle$ of the unperturbed Hamiltonian. The averaged distribution $P(r)$ is defined by $r=n-m$, and averaging over several states with roughly the same energy $E_{n}$ yields the averaged shape of eigenfunctions (ASoE). Alternatively, if regarded as a function of $n$ and averaging over several states around a given energy $E_{m}$, the kernel $P(r)$ represents up to some trivial scaling and shifting the local density of states (LDoS):

$$
P(E \mid m)=\sum_{n}\left|\left\langle n(k) \mid m\left(k_{0}\right)\right\rangle\right|^{2} \delta\left(E-E_{n}\right) .
$$

Its lineshape is fundamental for the understanding of the associated dynamics, which we will address in the next chapter, since its Fourier transform is the so-called "survival probability amplitude". In the following we will focus on the LDoS scenario.

The profile $P(r)$ undergoes a set of structural changes as a function of growing $\delta k$. We first summarize the generic picture, which involves the parametric scales $\delta k_{\mathrm{qm}}$ and $\delta k_{\mathrm{prt}}$, and the approximations $P_{\mathrm{FOPT}}, P_{\mathrm{prt}}$, and $P_{\mathrm{sc}}$. Then we discuss how to determine these scales, and what these approximations are.

- The standard perturbative - or first order perturbation theory (FOPT) - regime is defined as the range $\delta k<\delta k_{\mathrm{qm}}$ where we can use FOPT to get an approximation that we denote as $P() \approx P_{\text {FOPT }}$.

- The extended perturbative regime is defined as the range $\delta k_{\mathrm{qm}}<\delta k<\delta k_{\mathrm{prt}}$ where we can use perturbation theory (to infinite order) to get a meaningful approximation that we denote as $P() \approx P_{\text {prt }}$. Obviously $P_{\text {prt }}$ reduces to $P_{\mathrm{FOPT}}$ in the FOPT regime. 


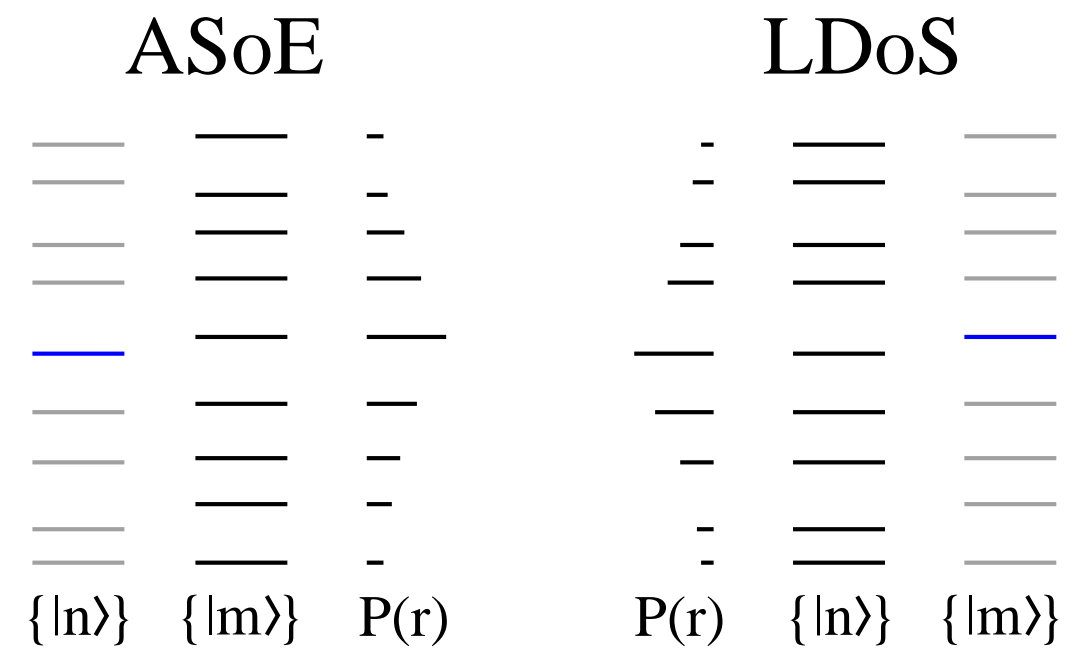

Figure 3.10.: Schematic representation of the two notions of the kernel $P(n \mid m)$. Left: projection of one perturbed eigenstate $\left|n\left(k_{0}+\delta k\right)\right\rangle$ (blue level) on the basis $\left|m\left(k_{0}\right)\right\rangle$ of the unperturbed Hamiltonian. Averaging over several $\left|n^{\prime}\right\rangle$ states around energy $E_{n}$ yields the averaged shape of eigenfunctions (ASoE). Right: alternatively, if $P(n \mid m)$ is regarded as a projection of one unperturbed eigenstate $|m\rangle$ (blue level) on the basis $|n\rangle$ of the perturbed Hamiltonian and averaged over several states around $E_{m}$, it leads to the local density of states (LDoS).

- The non-perturbative regime is defined as the range $\delta k>\delta k_{\text {prt }}$ where perturbation theory becomes non-applicable. In this regime we have to use either RMT or semiclassics in order to get an approximation that we denote as $P() \approx P_{\mathrm{sc}}$.

\subsection{Approximations for the profile $P(n \mid m)$}

The simplest case is obviously the FOPT regime where, for $P(n \mid m)$, we can use the standard textbook approximation $P_{\mathrm{FOPT}}(n \mid m) \approx 1$ for $n=m$, while

$$
P_{\mathrm{FOPT}}(n \mid m)=\frac{\delta k^{2}\left|\mathbf{B}_{m n}\right|^{2}}{\left(E_{n}-E_{m}\right)^{2}},
$$

for $n \neq m$. If the matrix elements outside of the band vanish $\mathbf{B}_{m n}=0$ (as in the WBRM model) then $P_{\mathrm{FOPT}}(r)=0$ for $|r|>b$. To find the higher order tails (outside of the band) we have to go to higher orders in perturbation theory. The above approximation (3.22) applies as long as only nearest-neighbor levels are mixed by the perturbation, i.e. $\delta k<\delta k_{\mathrm{qm}}$, where

$$
\delta k_{\mathrm{qm}}=\Delta / \sigma .
$$

If $\delta k>\delta k_{\mathrm{qm}}$ but not too large then we expect that several levels are mixed. This leads to a non-perturbative "core" of width $\Gamma$ which contains most of the probability and a tail region 
which is still described by FOPT while outside of the bandwidth the tails decay faster than exponentially. This is a non-trivial observation which can be justified by using perturbation theory to infinite order. It turns out that the non-perturbative mixing on the small scale of the core does not affect the long-range transitions [56]. Therefore we can argue that a reasonable approximation is [59]

$$
P_{\mathrm{prt}}(n \mid m)=\frac{\delta k^{2}\left|\mathbf{B}_{m n}\right|^{2}}{\left(E_{n}-E_{m}\right)^{2}+\Gamma^{2}},
$$

where the core-width $\Gamma$ is evaluated by imposing normalization of $P_{\text {prt }}(n \mid m)$. In the case of the WBRM model (flat bandwidth) we have

$$
\Gamma=\left(\frac{\sigma \delta k}{\Delta}\right)^{2} \times \Delta=\left(\frac{\delta k}{\delta k_{\mathrm{qm}}}\right)^{2} \times \Delta .
$$

and $P_{\text {prt }}(n \mid m)$ is the well-known Wigner Lorentzian [208, 209, 96]. In the general case the profile $P(n \mid m)$ can be described as a "core-tail" structure. For non-interacting systems with chaotic classical limit, recent studies [56,59] indicated that the above scenario (based on random matrix modeling) leads to a fairly good description of the kernel. Specifically, it was found [56] that the core-width scaled as

$$
\Gamma \sim \delta k^{\alpha} \quad \text { with } \quad 1<\alpha \leq 2 .
$$

This generalization of Eq. (3.25) defines the upper and the lower bound of the exponent $\alpha$. In order to derive this result one assumes a structureless core (i.e. only one scale $\Gamma$ is involved) which contains basically the entire probability.

The above approximation (3.24) makes sense only as long as we can still determine a core-tail structure, i.e. as long as $\Gamma(\delta k)<\Delta_{b}$. This expression assumes that the bandwidth $\Delta_{b}$ is sharply defined, as in the WBRM model. By elimination this leads to the determination of $\delta k_{\text {prt }}$, which in case of the WBRM model is simply

$$
\delta k_{\mathrm{prt}}=\sqrt{b} \delta k_{\mathrm{qm}} \sim \frac{\hbar}{\tau_{\mathrm{cl}} \sqrt{C(0)}} .
$$

In more general cases the bandwidth is not sharply defined. Then we have to define the perturbative regime using a practical numerical procedure. The natural definition that we adopt is as follows. We calculate the spreading $\delta E(\delta k)$, which is a linear function (see also the following section). Next we calculate $\delta E_{\text {prt }}(\delta k)$, using Eq.(3.24)). This quantity always saturates for large $\delta k$ because of having finite bandwidth. We compare it to the exact $\delta E(\delta k)$, and define $\delta k_{\text {prt }}$, for instance, as the $80 \%$ departure point.

What happens if perturbation theory completely fails? In the WBRM model the LDoS becomes semicircle [208, 209, 96]:

$$
P_{\mathrm{sc}}(n \mid m)=\frac{1}{2 \pi \Delta} \sqrt{4-\left(\frac{E_{n}-E_{m}}{\Delta}\right)^{2}} .
$$



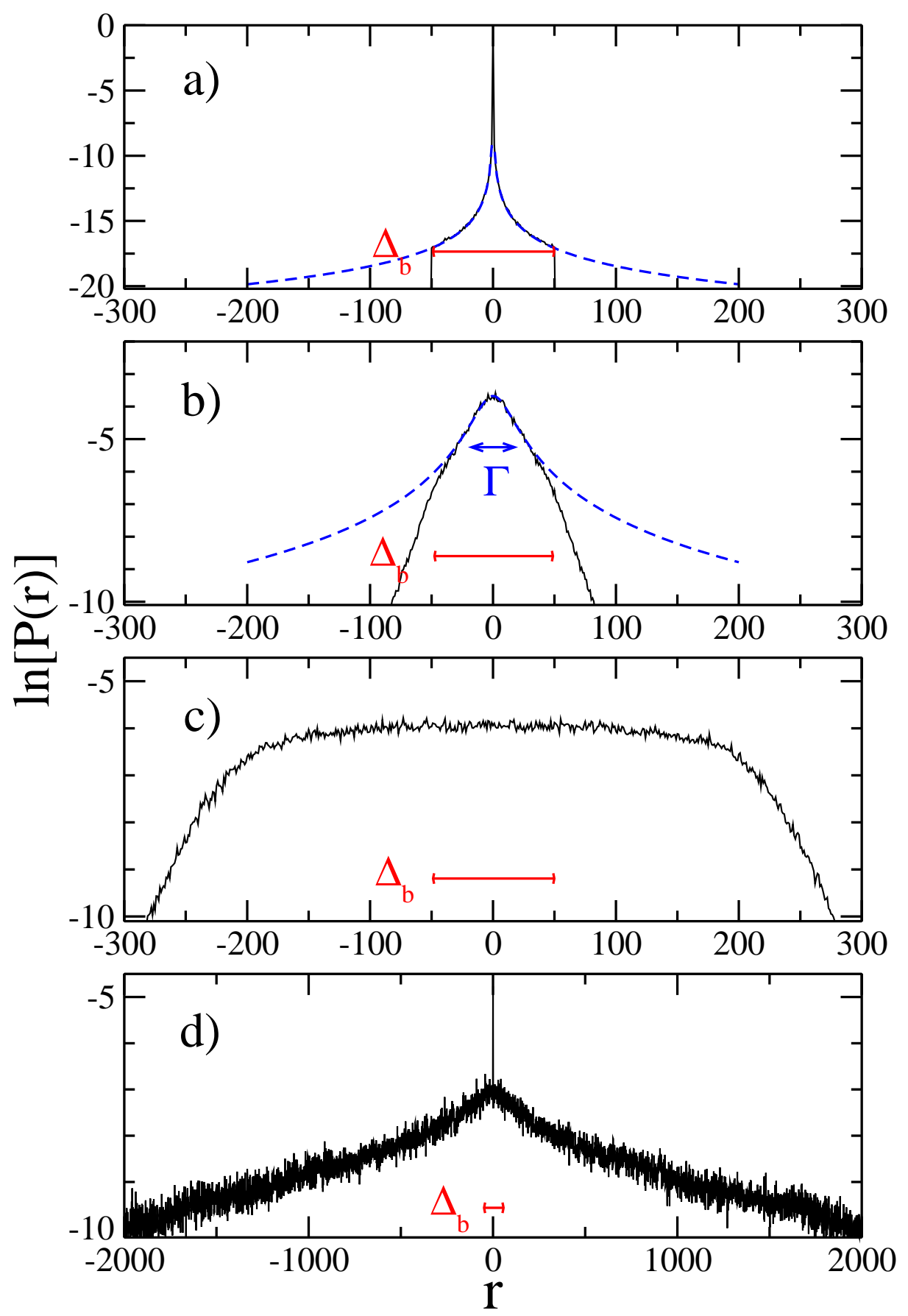

Figure 3.11.: The parametric evolution of eigenstates of a WBRM model with $\sigma=1$ and $b=50$ : (a) standard perturbative regime corresponding to $\delta k=0.01$, (b) extended perturbative regime with $\delta k=2$ (c) non-perturbative (ergodic) regime with $\delta k=12$ and (d) localized regime with $\delta k=1$. In (a-c) the mean level spacing $\Delta=1$ while in (d) $\Delta=10^{-3}$. The bandwidth $\Delta_{b}=\Delta \times b$ is indicated in all cases. In (b) the blue dashed line corresponds to a Lorentzian with $\Gamma \approx 16 \ll \Delta_{b}$ while in (a) we have $\Gamma \approx 10^{-4} \ll \Delta$ which therefore reduces to the standard FOPT result. 
If the perturbation strength is increased even further, the WBRM model displays a fourth regime which goes beyond the generic picture and we mention it for completeness only. It is the so-called "localization regime" which is found in the case of for $\delta k>\delta k_{\text {loc }}$ where

$$
\delta k_{\mathrm{loc}}=b^{3 / 2} \delta k_{\mathrm{qm}} .
$$

In this regime it is important to distinguish between the non-averaged $P(n \mid m)$ and the averaged $P(r)$ because the eigenfunctions are non-ergodic but rather localized. This localization is not reflected in the LDoS which is still a semicircle. A typical eigenstate is exponentially localized within an energy range $\delta E_{\xi}=\xi \Delta$ much smaller than $\delta E_{\mathrm{cl}}$. The localization length is $\xi \approx b^{2}$. In actual physical applications it is not clear whether there is such a type of localization. The above scenario for the WBRM model is summarized in Fig. 3.11 where we plot $P(n \mid m)$ in the various regimes. The localized regime is not an issue in the present work and therefore we will no further be concerned with it. Instead, we will now discuss the non-perturbative regime for dynamical models.

\subsection{Classical profile and quantum classical correspondence}

For systems that have a semiclassical limit we expect a qualitatively different behavior in the non-perturbative regime. Due to the strong perturbations many levels are mixed and hence the quantum nature becomes "blurred". We can then approximate the spreading profile by

$$
P_{\mathrm{sc}}(n \mid m)=\int \frac{d I d \varphi}{(2 \pi \hbar)^{d}} \rho_{n}(I, \varphi) \rho_{m}(I, \varphi),
$$

where $\rho_{m}(I, \varphi)$ and $\rho_{n}(I, \varphi)$ are the Wigner functions that correspond to the eigenstates $\left|m\left(k_{0}\right)\right\rangle$ and $|n(k)\rangle$ respectively. In the strict classical limit $\rho$ can be approximated by the corresponding micro-canonical distribution $\rho \propto \delta(E-\mathcal{H}(I, \varphi))$ which determines the energy surface $E$. The resulting classical LDoS profile $P_{\mathrm{cl}}(n \mid m)$ is obtained by its projection onto the energy surfaces $E_{n}$ of the perturbed system (and analogously for the ASoE). This is illustrated in Fig. 3.12 where the perturbed energy surfaces are depicted as black concentric circles while the initial preparation $E_{0}$ corresponds to the red circle. ${ }^{8}$ The overlap of the red and the black circles determines the classical profile $P_{\mathrm{cl}}(n \mid m)$. One expects a large overlap for tangential (singular) intersections while for larger angle (simple) intersections the overlap will be small. We note that outside the region indicated by blue vertical dashed lines the profile should vanish, displaying a sharp cut-off. This has to be seen in contrast to the quantum profile where these classically forbidden regions can be accessed since the Wigner functions possess a transversal width and thus smooth the edges of the quantum profile.

\footnotetext{
${ }^{8}$ Of course, in the classical system the surfaces are dense in the phase space and not separated as in the cartoon.
} 


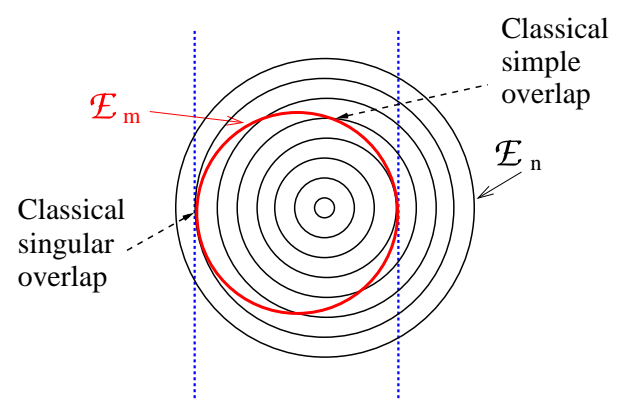

Figure 3.12.: Phase space illustration of the energy surfaces contributing to the classical profile $P_{\mathrm{cl}}(n \mid m)$ : the initially occupied energy surface $E_{m}$ (red circle) is projected onto the energy surfaces $E_{n}$ of the perturbed Hamiltonian $\mathcal{H}$. The classically forbidden region is outside the cut-off indicated by the blue vertical dashed lines. See text for details.

In order to calculate the profile $P_{\mathrm{cl}}(n \mid m)$ in practice, i.e., to evaluate the distribution of the projected energies $E_{n}$ one has to "fill" the initial energy shell $E_{m}$. This can be either done by generating a large set of initial conditions fulfilling $\mathcal{H}_{0}(I, \varphi)=E_{m}$ or, if the system is ergodic, by propagating one such initial condition for a sufficiently long time under $\mathcal{H}_{0}$. The latter method will be used later in the chapter, leading to the fluctuating energy $\mathcal{H}(I(t), \varphi(t))=E(t)$.

Irrespective of these structural changes of the spreading profile discussed above, it can be proved $[45,137]$ that the variance of the $\operatorname{LDoS}$

$$
\delta E=\sqrt{\sum_{n} P(n \mid m)\left(E_{m}^{(0)}-E_{n}\right)^{2}}
$$

of $P(r)$ is strictly linear and given by the expression

$$
\delta E(\delta k)=\sqrt{C(0)} \delta k \equiv \delta E_{\mathrm{cl}} .
$$

The only assumption that underlies this statement is $\delta k \ll \delta k_{\mathrm{cl}}$ and reflects the linear departure of the energy surfaces. In order to see the above relation (3.32) we calculate the variance $\delta E^{2}$ of $P(n \mid m)$ using the first two moments of the Hamiltonian in the unperturbed basis

$$
\begin{aligned}
\delta E^{2} & =\left\langle m\left|\hat{H}^{2}\right| m\right\rangle-\langle m|\hat{H}| m\rangle^{2}=\delta k^{2}\left[\left\langle m\left|\hat{B}^{2}\right| m\right\rangle-\langle m|\hat{B}| m\rangle^{2}\right] \\
& =\delta k^{2}\left[\sum_{n}\left|\mathbf{B}_{n m}\right|^{2}-\left|\mathbf{B}_{m m}\right|^{2}\right] .
\end{aligned}
$$

In other words, the quantum mechanical variance $\delta E^{2}$ is determined solely by the bandprofile $\left|\mathbf{B}_{n m}\right|^{2}$. On the other hand, the bandprofile is given by the semiclassical relation (3.14) leading to (3.32). As a consequence the variance $\delta E^{2}$ of the quantum and the classical spreading profile are identical irrespective of the different nature of the profile $P(n \mid m)$.

This behavior is the so-called restricted quantum-classical correspondence (QCC) [59] because it applies only to the second moment $\delta E^{2}$ of the energy spreading. In contrast, if the entire profiles $P(n \mid m)$ and $P_{\mathrm{cl}}(n \mid m)$ match we use the term detailed QCC . 


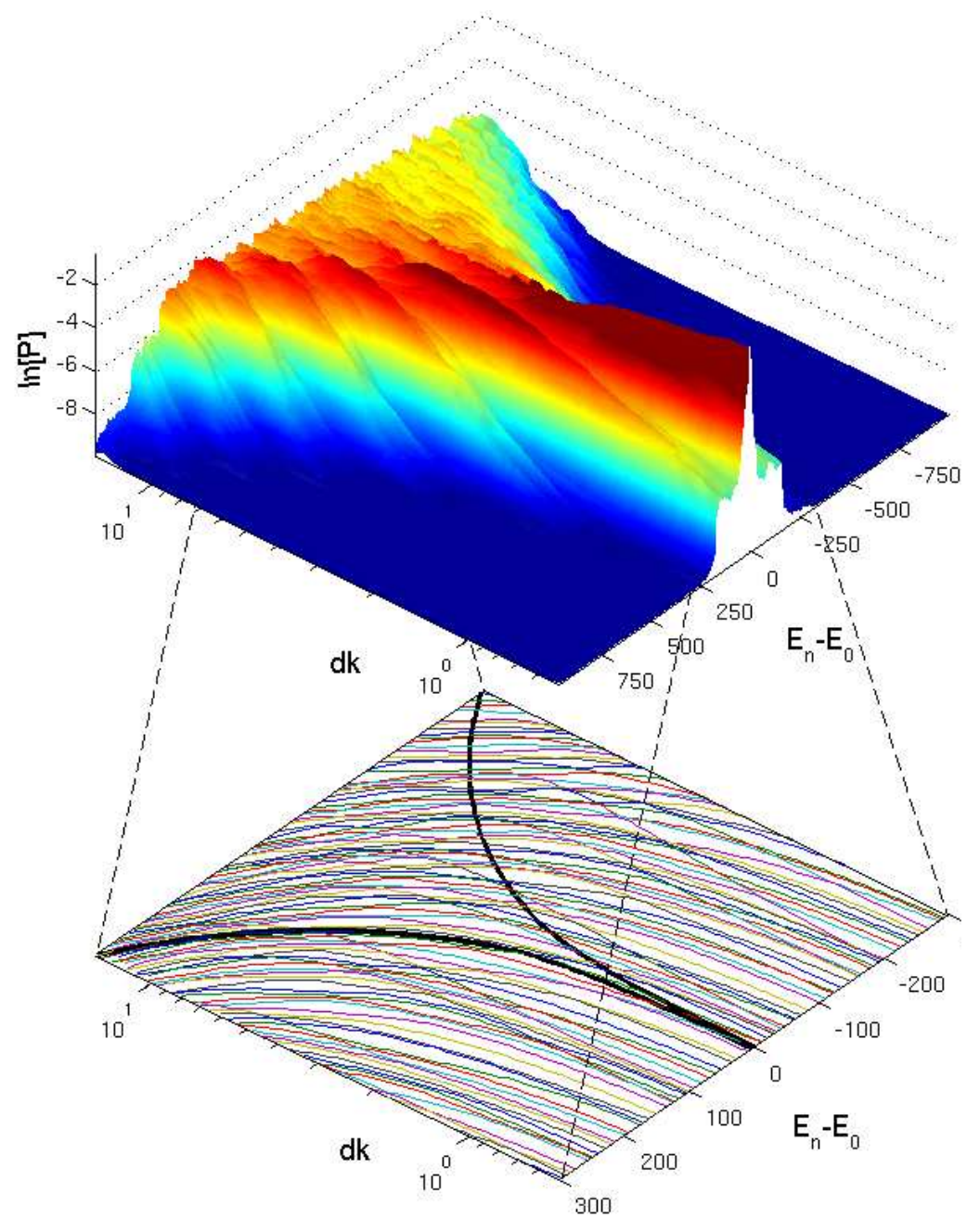

Figure 3.13.: The kernel $P(n \mid m)$ of the BHH plotted as a function of the perturbed energies $E_{n}$ (LDoS representation) and for various perturbation strengths $\delta k>\delta k_{\mathrm{qm}}$. In the lower plane we report the parametric evolution of the energy levels within the bandwidth $\Delta_{b} \approx 280$. The energy width $\Gamma$ is shown (bold line) as a function of $\delta k$. The averaged shape of eigenfunctions is given by the same kernel $P(n \mid m)$ and is obtained by just inverting the energy axis. Here, $N=70$, and $\lambda_{0}=0.053$.

\subsection{The $P(n \mid m)$ for the Bose-Hubbard Hamiltonian}

Does our BHH model follow the same scenario or will the interatomic interactions affect the shape of $P(n \mid m)$ ? An overview of the parametric evolution of the averaged $P(n \mid m)$ is shown in Fig. 3.13. Beginning as a delta function for $\delta k=0$, the profile $P(n \mid m)$ starts to develop a non-perturbative core as $\delta k>\delta k_{\mathrm{qm}}$, which eventually spills over the entire bandwidth $\Delta_{b}$. The other striking feature is the appearance of sidebands in the profile. In the following we present a detailed analysis of the various parametric regimes. 


\subsubsection{The perturbative regimes}

We start with the discussion of the perturbative regimes; the corresponding data are presented in Figs. 3.14a, b where we show $P(n \mid m)$ for the BHH and the IRMT model together with the perturbative profile $P_{\text {prt }}(n \mid m)$ obtained from Eqs. (3.22) and (3.24). All three curves show an excellent agreement both in the standard and extended perturbative regime. ${ }^{9}$

At the same time one observes the appearance of pronounced sidebands in the profile $P(n \mid m)$. They originate from the structure in the energy landscape of the perturbation matrix $\mathbf{B}$ as can be verified by direct inspection of Fig. 3.7. Formally, this can be seen from the perturbation theory where the information about the bandprofile enters through the numerator in Eqs. (3.22) and (3.24). Consequently, the line-shape of the averaged wavefunction $P(n \mid m)$ is different from Lorentzian (see Fig. 3.14b) which would be expected for the WBRM model that is characterized by a flat bandprofile. Still, the general features of $P_{\text {prt }}(n \mid m)$, i.e. the core-tail structure, can be detected. ${ }^{10}$

In order to quantitatively characterize the profile $P(n \mid m)$ of the BHH model we will employ various measures. Our first candidate is the energy spreading $\delta E$ which is plotted in Fig. 3.15a as a function of the perturbation strength $\delta k$. Interestingly, the structural changes of the profile $P(n \mid m)$ are not reflected at all in the spreading $\delta E$. On the other hand, this should not be too surprising since we have found an analytical expression (see Eq. (3.33)) indicating that the standard deviation $\delta E$ of the LDoS profile increases linearly with $\delta k$ (the dashed black line has slope one and is drawn to guide the eye). Even more, we could show that $\delta E$ should be equal to the classical spreading $\delta E_{\mathrm{cl}}$ (blue line). This is confirmed by the numerics and represents a manifestation of the above mentioned restricted QCC which persists throughout the regimes.

In order to detect the appearance of a core we have to inquire a measure that is sensitive to the structure of the profile like the core-width $\Gamma$. Looking at the corresponding curve (orange line) we see that the core is initially below the mean levels spacing $\Delta$ and one order of magnitude smaller than the energy spreading $\delta E$. As soon as we enter the extended perturbative regime, the core grows following a power-law

$$
\Gamma \propto\left(\delta k \frac{\sigma}{\Delta}\right)^{\alpha} \times \Delta \quad \text { with } \quad \alpha=1.9 \pm 0.1
$$

Our numerical data are thus in agreement with the theoretical prediction of Eq. (3.26). The dash-dotted line in Fig. 3.15 is drawn to guide the eye and has slope two. In the following we will assume $\alpha=2$.

Next, we determine the borders $\delta k_{\mathrm{qm}}, \delta k_{\mathrm{prt}}$ of the BHH model. The former is defined by the condition $\Gamma=\Delta$, i.e., the core-width to be of the size of the mean level spacing. Inserting Eqs. (3.5) and (3.14) into this condition we get

\footnotetext{
${ }^{9}$ We note that in the perturbative regimes, the RMT strategy fails in the far tails regime $\Delta \times|r|>\Delta_{b}$ where system specific interference phenomena become important (not shown).

${ }^{10}$ In a sense, Wigner's Lorentzian (3.24) is a special case of core-tail structure.
} 


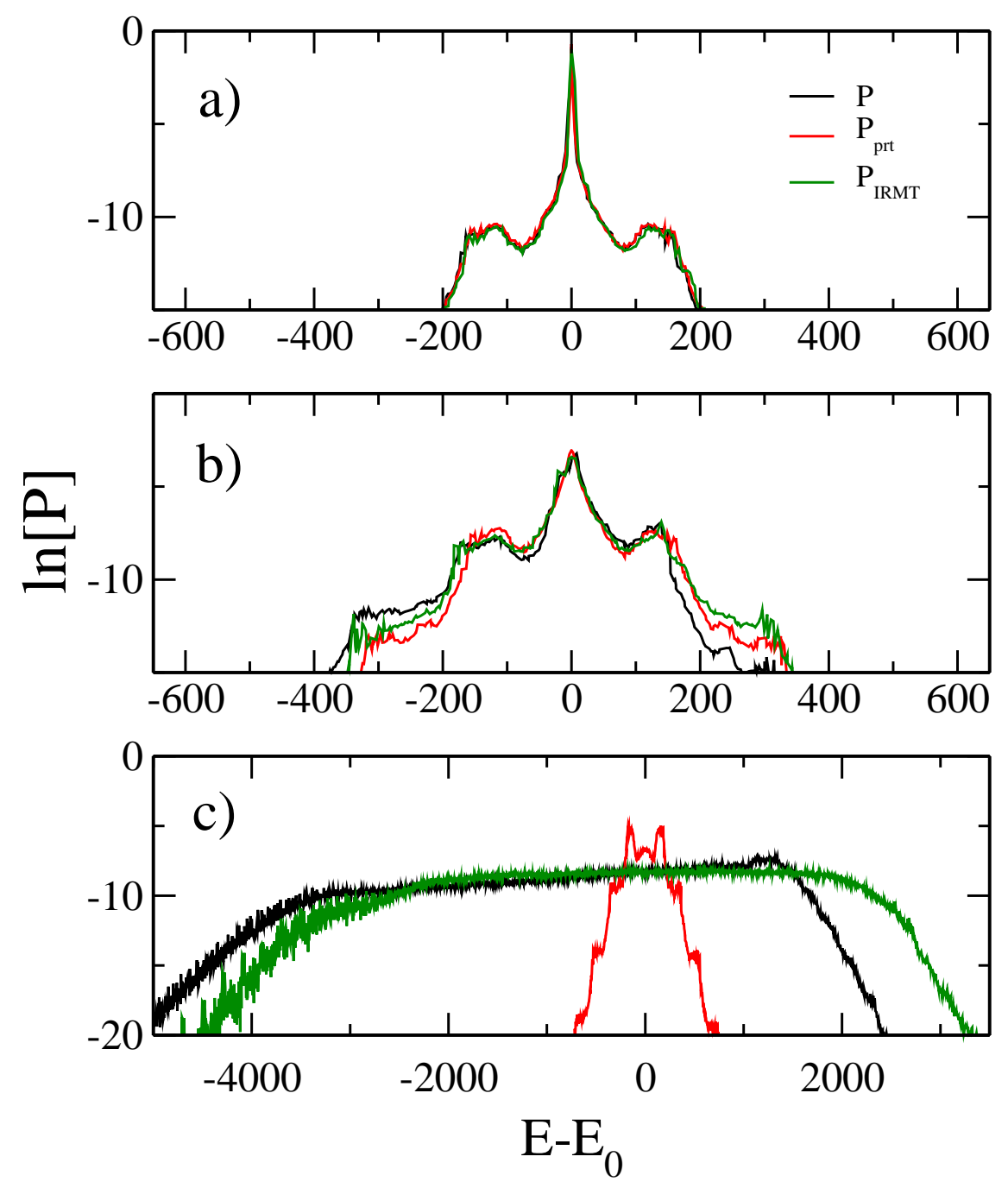

Figure 3.14.: The quantal profile $P(n \mid m)$ as a function of $E_{n}-E_{m}^{(0)}$ for the BHH model is compared with $P_{\text {prt }}$ and with the corresponding $P_{\mathrm{RMT}}$ of the IRMT model. The perturbation strength $\delta k$ is in (a) standard perturbative regime $\delta k=0.05$, (b) extended perturbative regime $\delta k=0.3$ and (c) non-perturbative regime $\delta k=10$. The system corresponds to $N=230, \tilde{U}=280$ and $k_{0}=15$. Here $\delta k_{\mathrm{qm}}=0.09$ and $\delta k_{\mathrm{prt}}=1.02$.

$$
\delta k_{\mathrm{qm}} \propto \frac{\tilde{U}}{N^{3 / 2}} .
$$

From the above discussion it should be clear that the notion of a core-width $\Gamma$ is meaningful only as long as we have $\Gamma<\Delta_{b}$, i.e. as long as we can distinguish a core-tail structure. By using this condition we determine the perturbative border $\delta k_{\text {prt }}$ to be 

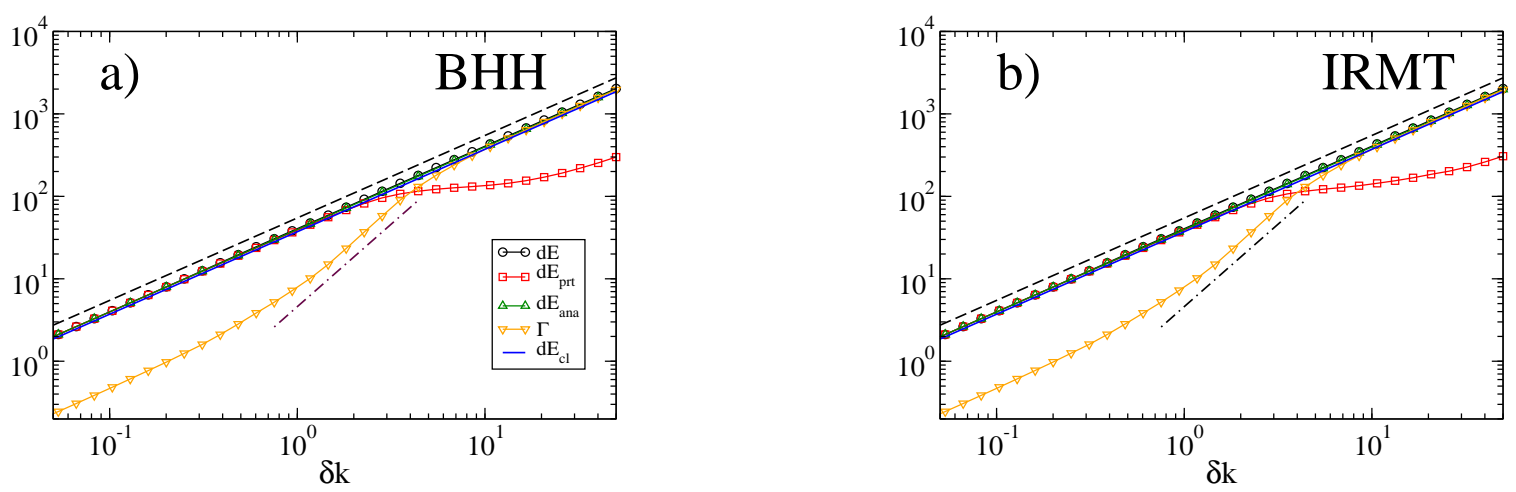

Figure 3.15.: Various measures of the spreading profile for the BHH and the IRMT model: the quantal spreading $\delta E$ (black line), the quantal spreading $\delta E_{\text {prt }}$ of the perturbative profile given by Eq. 3.24 (red line), the analytical spreading $\delta E_{\text {ana }}$ obtained from (3.33) (green line), the core-width $\Gamma$ (orange line), and the classical spreading $\delta E_{\mathrm{cl}}$ (blue line). The dashed line has slope one, while the dash-dotted line has slope two and are drawn to guide the eye. The systems correspond to $N=70$ bosons, $k_{0}=15$ and $\tilde{U}=280$. See text for details.

$$
\delta k_{\mathrm{prt}} \propto \frac{\tilde{U}}{N} .
$$

The numerical data for these scalings are reported in Fig. 3.16 and a nice agreement with the theoretical expectation is observed. ${ }^{11}$

Although the above calculations have been performed for the case $f=3$ they can be carried over to an arbitrary number of sites $f$. To this end, we recall that the Hilbert space dimension $\mathcal{N}$ grows as $\mathcal{N} \sim N^{f-2}$ (see Eq. 2.38) while the energy is $E=$ const. $\times \tilde{U} N$. Accordingly, the mean level spacing behaves as $\Delta \sim \tilde{U} / N^{f-2}$. With the standard deviation $\sigma$ of matrix elements $\mathbf{B}_{n m}$ being ${ }^{12} \sigma=N \sqrt{\Delta / \tilde{U}}$, we find

$$
\delta k_{\mathrm{qm}} \propto \frac{\tilde{U}}{N^{f / 2}} .
$$

In order to calculate $\delta k_{\text {prt }}$ we insert this result into (3.25) and get back Eq. (3.36), i.e. for larger lattices we expect a growing extended perturbative regime at the cost of a decreasing FOPT regime.

As mentioned above, we can alternatively determine $\delta k_{\text {prt }}$ as the perturbation strength where $\delta E_{\text {prt }}$ deviates from the linearly growing $\delta E$. In Fig. 3.15 we plot the energy spreading $\delta E_{\mathrm{prt}}$ of the perturbative profile as a red line. We find that this criterion yields the same

\footnotetext{
${ }^{11}$ In our numerical analysis we have defined, $\delta k_{\mathrm{qm}}$ as the perturbation strength for which $50 \%$ of the probability remains at the original site but we have checked that the condition $\Gamma=\Delta$ gives the same result. For determining $\delta k_{\text {prt }}$ we use the procedure described in the paragraph around Eq. (3.27).

${ }^{12}$ Note that the prefactor $N^{2} / \tilde{U}$ in (3.14) is a consequence of the rescaled variables introduced in Chapter 2 and hence of classical nature.
} 

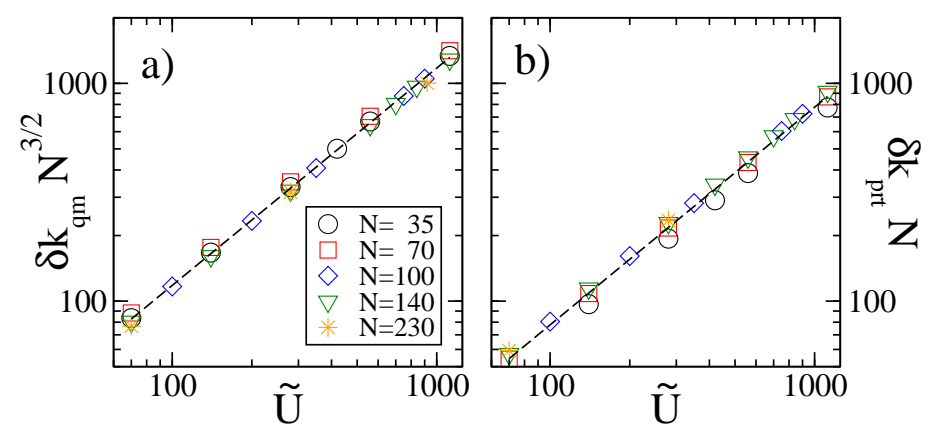

Figure 3.16.: The parameters (a) $\delta k_{\mathrm{qm}}$ and (b) $\delta k_{\mathrm{prt}}$ for various $\tilde{U}, N$, and for $\lambda_{0}=0.053$. A nice scaling in accordance with Eqs.(3.35) and (3.36) is observed.

results as the previously discussed condition, namely that the core-width $\Gamma$ becomes comparable to the bandwidth $\Delta_{b} \approx 280$. This indicates that both criteria are equivalent. We note that the increase of $\delta E_{\text {prt }}$ after the breakdown of perturbation theory for large $\delta k$ values is trivially caused by an increasing $\Delta$.

\subsubsection{The non-perturbative regime and detailed quantum-classical correspondence}

Next, we focus on the applicability of the RMT approach in the non-perturbative regime $\delta k>\delta k_{\text {prt }}$. Looking at the energy spreading $\delta E$ reported in Fig. 3.15b we observe that the random matrix model produces basically identical results as the BHH for all values of $\delta k$. Thus, we might be tempted to conclude that the IRMT model yields the correct profile $P(n \mid m)$ also for $\delta k>\delta k_{\text {prt }}$. Looking at Fig. 3.14c it is obvious that this is not the case: While the LDoS of the IRMT model approaches a semicircle, the profile $P(n \mid m)$ of the $\mathrm{BHH}$ becomes system-specific. Why is this not reflected in the spreading $\delta E$ ? The answer lies again in the nature of the variance $\delta E^{2}$. The latter is determined solely by the bandprofile $\left|\mathbf{B}_{n m}\right|^{2}$ through expression (3.33) which we overplot in Fig. 3.15 as a green line. One observes an excellent agreement with the numerically obtained $\delta E$ for both the BHH and the IRMT model which share by construction the same bandprofile. It is therefore clear, that we cannot distinguish between the models by looking at the spreading $\delta E .^{13}$

Coming back to the profile $P(n \mid m)$, the failure of the RMT approach can now be understood also from a different point of view. In Section 3.5, we had argued in a heuristic manner that the non-perturbative and the semiclassical limit coincide. Now, we can see this formally by looking at the scaling (3.36) of the perturbative border $\delta k_{\text {prt }} \sim \tilde{U} / N$ : One can either approach the non-perturbative limit by increasing the perturbation strength $\delta k$ or, alternatively, by fixing $\delta k$ and increasing $N$. As we have seen before increasing $N$ means to approach the semiclassical limit (keeping $\tilde{U}=$ const.). But the IRMT model does not have a semiclassical limit! Therefore, we cannot expect it to yield a correct description of

\footnotetext{
${ }^{13}$ As we shall see in the next chapter, this is not true for the time-dependent $k(t)$.
} 


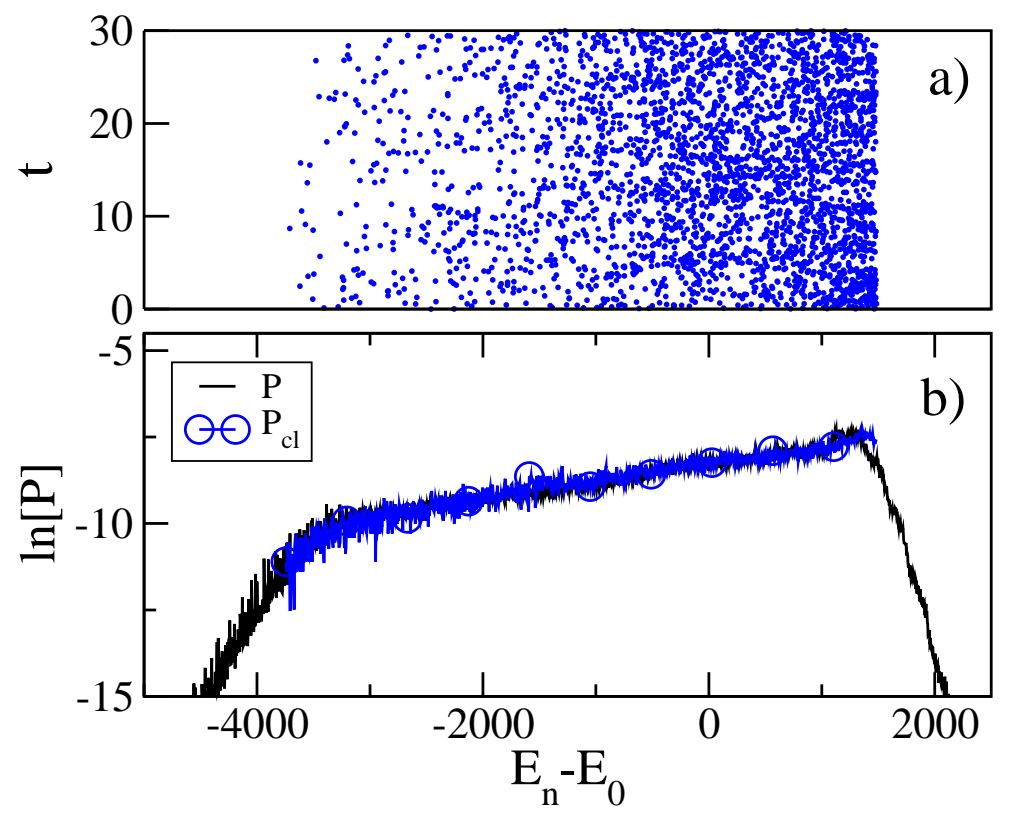

Figure 3.17.: The kernel $P(n \mid m)$ (LDoS representation) for the BHH in the non-perturbative regime $\delta k=10$ for $N=230$ and $\lambda=0.053$. In the upper panel we plot a time series $E(t)$ which leads to the classical profile $P_{\mathrm{cl}}(E)$ (see text for details).

$P(n \mid m)$ in that regime. Instead, the LDoS becomes eventually semicircle (see Eq. (3.28)), irrespective of the exact bandprofile $\left|\mathbf{B}_{n m}\right|^{2}$ because higher order correlations of the true matrix elements $\mathbf{B}_{n m}$ are not taken into account by RMT.

What happens to the BHH in this regime? Since for $\delta k>\delta k_{\text {prt }}$ perturbation theory - even to infinite order - breaks down one has to rely on completely non-perturbative methods to evaluate $P(n \mid m)[56,59,112]$. As described in Section 3.5 we therefore propagate a trajectory $(I(t), \varphi(t))$ of the unperturbed Hamiltonian $\mathcal{H}_{0}$ and project it onto $\mathcal{H}$. In Fig. 3.17a we plot the resulting $E(t)=\mathcal{H}(I(t), \varphi(t))$ as a function of time. The classical distribution $P_{\mathrm{cl}}(n \mid m)$ is constructed (Fig. 3.17b) from $E(t)$, by averaging over a sufficiently long time. The good agreement with the quantum profile $P(n \mid m)$ is a manifestation of the detailed quantum-classical correspondence which - in contrast to the restricted QCC - is limited to the non-perturbative, i.e. semiclassical, regime. Note though that the classical profile $P_{\mathrm{cl}}(n \mid m)$ displays sharp borders as expected from the phase space picture discussed in Section 3.5.

\subsection{Conclusions}

In the first part of this chapter, we have studied the spectral properties of the trimeric $\mathrm{BHH}$ and related them to the nature (chaotic/regular) of the underlying classical dynamics. We then explored the structures in the energy landscape of the perturbation operator $\hat{B}$ corre- 
sponding to a change in the coupling strength $k_{0} \rightarrow k_{0}+\delta k$ between the neighboring sites and used the results to introduce an improved random matrix theory (IRMT) model. In the main part, we analyzed the structural changes which the eigenstates undergo as the perturbation strength $\delta k$ is increased, for both the BHH and the IRMT model. For $\delta k<\delta k_{\mathrm{qm}}$ the perturbation mixes only neighboring levels: The main component of the kernel $P(n \mid m)$ remains unaffected while corrections are captured by standard textbook finite order perturbation theory. For $\delta k_{\mathrm{qm}}<\delta k<\delta k_{\mathrm{prt}}$ a non-trivial structure appears, consisting of two distinct components: While the tails are still captured by perturbation theory, the central part is of non-perturbative nature and extends over an energy width $\Gamma \propto N^{2} \cdot \delta k^{2} / \tilde{U}$. In this case, the power spectrum of the generalized force $\tilde{C}(\tilde{\omega})$ is an important ingredient for the theory. Experimentally, it is directly measurable [217] because the momentum distribution of atoms in a lattice is $\sim \sum_{j} \exp (i k j) X_{j}$ where $k$ is the atomic momentum and $X_{j}=\left\langle\left[\hat{b}_{j+l}^{\dagger} \hat{b}_{l}+\right.\right.$ h.c. $\left.]\right\rangle$ is the one-particle density matrix. In the opposite limit $\delta k>\delta k_{\text {prt }}$ one can apply semiclassical (non-perturbative) considerations. For $\delta k>\delta k_{\text {prt }}$, random matrix theory breaks down and quantum mechanical perturbation theory fails totally. Instead, classical calculations can be used to predict the shape of $P(n \mid m)$. 


\section{Wavepacket Dynamics in Energy Space}

In recent years there has been an increasing interest in understanding the theory of driven quantum systems in fields as diverse as mesoscopic electronic, atomic and molecular physics [210, 211, 213, 40, 206, 212, 52, 58, 214, 138, 60, 112]. Driven systems are described by parametric Hamiltonians $\mathcal{H}(I, \varphi, X(t))$ with $(I, \varphi)$ being a set of canonical coordinates while $X(t)$ is some time dependent parameter. As a consequence of the time-dependence, the energy of the system is not a constant of motion but the system makes "transitions" between energy levels. In case of linear or periodic driving, the long time behavior of the energy spreading is characterized by diffusion, which results in a systematic increase of the average energy. This irreversible process of energy absorption is known as dissipation.

Currently a well developed classical theory of dissipation is available. On the quantum side, however, things are less clear. For example, questions on the validity of linear response theory and the implications of underlying classically chaotic dynamics have not been answered in a satisfactory way. In this respect, the first step towards understanding the quantum dissipation problem is to investigate the short time dynamics associated with simplified dynamical scenarios like the so-called wavepacket dynamics. Here cold bosons in optical lattices - as described by the Bose-Hubbard Hamiltonian - are viable candidates to experimentally realize such driven systems and study their quantum dynamics. This is due to the fact that they are readily created using standard techniques while at the same time the control in preparation and measurement of the atomic cloud is very precise.

Remarkably enough, there has already been an experimental observation of the manybody quantum dynamics for the special case of the integrable Bose-Hubbard dimer, with intriguing results [1] like symmetry-breaking and self-trapping of the boson population. However, for the more general scenario of chaotic Bose-Hubbard Hamiltonians less is known. Its spectral properties [71, 64, 49, 136] have been studied earlier in the context of molecular physics, while only recently the eigenfunctions [115] were investigated. Apart from Refs. [89, 166], where the dynamics of a model similar to the BHH was considered, most of the works rely on semiclassical or mean-field treatments like the Gross-Pitaevskii equation $[41,92,93]$.

In this chapter, we will study the response of cold atoms to a rectangular pulse of finite duration $t$ that perturbs the coupling between adjacent wells of the chaotic BHH. The corresponding dynamical scenario is known as wavepacket dynamics [52, 57, 137, 115], and represents the natural continuation of Chapter 3. There we studied the "sudden" case (LDoS) where no time evolution takes place after the system is perturbed.

Wavepacket dynamics is one of the most basic non-trivial dynamical evolution schemes and therefore of fundamental interest. At the same time, its analysis will pave the way 
for understanding more demanding evolution scenarios and ultimately the response of cold atoms under persistent driving. The main target of this study is to identify the limits of the various theoretical approaches to the problem. On the one hand, we will use linear response theory (LRT) which constitutes the leading framework for the analysis of driven systems. Considering at the same time quantum and classical LRT we will pay special attention to the manifestation of detailed vs. restricted quantum classical correspondence (QCC) as introduced in Chapter 3. On the other hand, we are going to investigate the limits of the random matrix theory (RMT) modeling. In the previous chapter RMT proved to capture the spectral statistics as well as the LDoS shape in the perturbative regime. Note, however, that the applicability of RMT is a conjecture which should be tested. While the last chapter was focused on static properties, we here study wavepacket dynamics. This poses a bigger challenge to RMT since the dynamical evolution involves both eigenvalues and eigenvectors as well as correlations between them.

The structure of this chapter is as follows: In the next section we discuss the setup of the wavepacket dynamics experiment together with the different measures which we will employ to investigate it and mark the general assumptions underlying the study. In Section 4.2 we present the theoretical calculations for driven quantum systems based on LRT and revise the knowledge on the dynamical evolution of chaotic quantum single-particle systems $[52,57,112]$. In the same section we show how the survival probability is connected to the LDoS which was studied in the previous chapter. Section 4.3 contains the results of our numerical analysis [116]. We find a good agreement with the theoretical predictions obtained via LRT, and also observe quantum classical correspondence. We demonstrate the weakness of the RMT strategy in the regime of strong perturbations. Finally, we exhibit a striking feature of the lattice dynamics in the so-called self-trapped regime. The last section summarizes our findings.

\subsection{Preliminary considerations and object of the study}

In this chapter we study the time evolution of the energy distribution in the Bose-Hubbard Hamiltonian as the coupling strength $k=k(t)$ between neighboring sites is changed. ${ }^{1} \mathrm{We}$ are going to use the trimeric $\mathrm{BHH}^{2}$ (see Section. 2.6)

$$
\hat{H}=\hat{H}_{0}-\delta k(t) \hat{B},
$$

where the unperturbed Hamiltonian $\hat{H}_{0}$ is given by Eq. (2.39) with $k=k_{0}=k(0)$ and $\hat{B}$ being the coupling operator (see Eq. 3.2). Again, we emphasize that a fixed assumption of this work is that the perturbation $\delta k(t)=k(t)-k(0)$ is classically small $\delta k \ll \delta k_{\mathrm{cl}}$, and thus we are always in the classical LRT regime. If not stated otherwise, we will use the

\footnotetext{
${ }^{1}$ Experimentally, this type of perturbation is easily implemented (see Chapter 2 ).

${ }^{2}$ Again, we stress the fact that for an appropriate choice of energy and lattice parameters the BHH trimer can be chaotic and thus contains the main ingredient to generalize the result to larger lattices.
} 


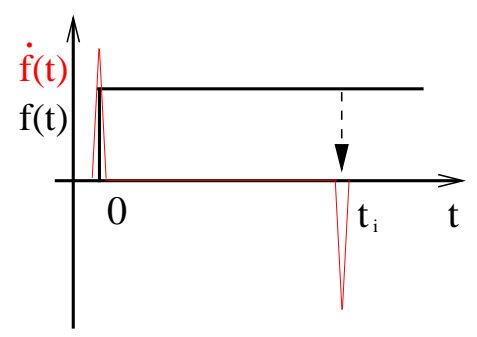

Figure 4.1.: Scheme of the wavepacket dynamics scenario: the perturbation is a rectangular pulse of duration $t_{i}$ at which the measurement is done. The function $f(t)$ represents the rescaled time-dependence of the perturbation $\delta k(t)=\delta k \times f(t)$ (black line) while the red line indicates its time derivative $\dot{f}(t)$.

same parameters as in the previous chapter, i.e., $k_{0} \approx 15, \tilde{U} \approx 280$, and an energy window $\tilde{H} \approx 0.26 \pm 0.02$, where the motion is predominantly chaotic.

As before, the calculations are carried out in the basis of $\hat{H}_{0}$ in which the latter becomes diagonal, i.e. $\mathbf{H}=\mathbf{E}_{\mathbf{0}}-\delta k(t) \mathbf{B}$ (see also Eq. (3.3)). In contrast to the LDoS studies, $\delta k(t)$ is now time dependent. For later purposes it is convenient to write the perturbation as

$$
\delta k(t)=\delta k \times f(t),
$$

where $\delta k$ controls the "strength of the perturbation" while $f(t)$ is the scaled time dependence $^{3}$.

Although our focus will be on the wavepacket dynamics scenario where the perturbation is a rectangular pulse of strength $\delta k$ and duration $t$ - see Fig. 4.1 for a sketch of the resulting step function $f(t)$ with $k(t)=k(0)$ - we expect that the results will shed some light on the more demanding problem of quantum dissipation.

In order to study the dynamics, we have to introduce measures that quantify the departure from the initial state. We define a set of such measures in the following subsection.

\subsubsection{Measures of the evolving distribution $P_{t}\left(n \mid n_{0}\right)$}

We consider an initial micro-canonical preparation in an eigenstate $\left|n_{0}\right\rangle$ of the unperturbed Hamiltonian $\hat{H}(I, \varphi ; k(0))$. Given the driving scenario $k(t)$, it is most natural to analyze the evolution of the probability distribution

$$
P_{t}\left(n \mid n_{0}\right)=\left|\left\langle n|\hat{U}(t)| n_{0}\right\rangle\right|^{2},
$$

where

$$
\hat{U}(t)=\hat{T} \exp \left[-\frac{i}{\hbar} \int_{0}^{t} \mathrm{~d} t^{\prime} \hat{H}\left(k\left(t^{\prime}\right)\right]\right.
$$

is the time-ordered evolution operator and $\hat{H}(I, \varphi ; k(t))|n\rangle=E_{n}|n\rangle$. Note that the LDoS (3.20) is recovered from the above kernel (4.3) by setting the time evolution to $\hat{U}(0) \approx 1$.

By convention we order the states by their energy. Hence we can regard $P_{t}\left(n \mid n_{0}\right)$ as a function of $r=n-n_{0}$, and average over the initial preparation, so as to get a smooth distribution $P_{t}(r)$.

\footnotetext{
${ }^{3}$ Note that if we had $f(t) \propto t$, i.e. persistent driving, then $\delta k$ would be the "rate" of the driving.
} 
The survival probability is defined as

$$
P(t)=\left|\left\langle n_{0}|\hat{U}(t)| n_{0}\right\rangle\right|^{2}=P_{t}\left(n_{0} \mid n_{0}\right),
$$

and the energy spreading is defined as

$$
\delta E(t)=\sqrt{\sum_{n} P_{t}\left(n \mid n_{0}\right)\left(E_{n}-E_{n_{0}}\right)^{2}} .
$$

These are the major measures for the characterization of the distribution we are going to analyze in this chapter.

The physics of $\delta E(t)$ is very different from the physics of $P(t)$ : While the former is very sensitive to the tails of the distribution, the latter probes only the initial excitation $\left|n_{0}\right\rangle$. Yet, the actual "width" of the distribution is not captured by any of these measures: As we have seen in Chapter 3 a "core" of width $\Gamma$ (see Eq. 3.34) can appear as a result of a nonperturbative mixing of levels. Similarly to the $\Gamma$ which was obtained from normalization of the perturbative profile we can define an operative measure $\delta E_{\text {core }}$ that reflects the width of the "core" of the distribution which contains $50 \%$ of the probability:

$$
\delta E_{\text {core }}(t)=\left[n_{75 \%}-n_{25 \%}\right] \Delta .
$$

Here, $\Delta$ is the mean level spacing and $n_{q}$ is determined through the equation $\sum_{n} P_{t}\left(n \mid n_{0}\right)=q$.

As an additional characteristic of the distribution we introduce the participation ratio $\delta n_{\mathrm{IPR}}(t)$. It yields the number of levels occupied by the distribution at time $t$ and is defined as

$$
\delta n_{\mathrm{IPR}}=\left(\sum_{n}\left|c_{n}\right|^{4}\right)^{-1},
$$

where $\left|c_{n}\right|^{2}=P_{t}\left(n \mid n_{0}\right)$. The ratio $\delta n_{\mathrm{IPR}} /\left(n_{75 \%}-n_{25 \%}\right)$ can be used as a measure for sparsity. We consider in this chapter strongly developed chaos, so that sparsity is not an issue and $\delta n_{\mathrm{IPR}} \sim \delta E_{\text {core }} / \Delta$.

\subsection{Linear response theory}

In this section we derive the analytical calculations for the quantum dynamics using linear response theory. The strategy is to identify regimes in a way similar to the LDoS theory described in Chapter 3. However, the definition of regimes for driven systems is more complicated: It is clear that for short times we can always use time-dependent FOPT. The question is, of course, what happens next. Here we have to distinguish between two different scenarios. The first one is wavepacket dynamics for which the dynamics is a transient from a preparation state to some new ergodic state. The second scenario is persistent driving, either linear $(\delta k(t)=\delta k t)$ or periodic $(\delta k(t)=\delta k \sin (\Omega t))$. In the latter case the strength of the perturbation depends also on the rate of the driving, not just on the amplitude. We will 
keep the resulting LRT expressions as general as possible and apply them to the wavepacket dynamics in the next section. This will allow us to draw some general conclusions as far as driven systems are concerned.

The crucial question is on the validity of linear response theory[112]. In order to avoid ambiguities we here adopt a practical definition. Whenever the result of the calculation depends only on the two point correlation function $C(\tau)$ (see Eq. (3.11)), or equivalently only on the bandprofile of the perturbation (which is described by $\tilde{C}(\omega)$, see Eq. (3.14)), then we refer to it as "LRT". This implies that higher order correlations are not expressed. There is a (wrong) tendency to associate LRT with FOPT. In fact the validity of LRT is not simply related to FOPT. We shall clarify this issue in the next subsection.

For both $\delta E(t)$ and $P(t)$ we have "LRT formulas" which we discuss in the next subsections. Writing the driving pulse as $\delta k(t)=\delta k f(t)$ we obtain for the energy spreading

$$
\delta E^{2}(t)=\delta k^{2} \times \int_{-\infty}^{\infty} \frac{d \omega}{2 \pi} \tilde{C}(\omega) \tilde{F}_{t}(\omega),
$$

while for the survival probability we have

$$
\mathcal{P}(t)=\exp \left[-\delta k^{2} \times \int_{-\infty}^{\infty} \frac{d \omega}{2 \pi} \tilde{C}(\omega) \frac{\tilde{F}_{t}(\omega)}{(\hbar \omega)^{2}}\right] .
$$

Two spectral functions are involved: One is the classical power spectrum $\tilde{C}(\omega)$ of the fluctuations defined in Eq. (3.12), and the other $\tilde{F}_{t}(\omega)$ is the spectral content of the driving pulse which is defined as

$$
\tilde{F}_{t}(\omega)=\left|\int_{0}^{t} d t^{\prime} \dot{f}\left(t^{\prime}\right) \mathrm{e}^{-i \omega t^{\prime}}\right|^{2} .
$$

Since this is the only information which enters the perturbation theory we expect that a theoretical modeling that incorporates both $\tilde{F}_{t}(\omega)$ and $\tilde{C}(\omega)$ but does not contain higher order correlations will reproduce exactly the LRT results. In Section 3.2 we introduced such a strategy which we follow here as well. Namely, we will model the dynamics generated by the BHH using the improved RMT (IRMT) model that incorporates $\tilde{C}(\omega)$ through the bandprofile of the perturbation matrix $\mathbf{B}$ while the spectral content $\tilde{F}_{t}(\omega)$ is imposed by the driving. We will test the applicability of RMT and pay special attention to the behavior of the IRMT dynamics in the non-perturbative regime.

Here we summarize the main observations regarding the nature of wavepacket dynamics in the various regimes:

- FOPT regime: In this regime $\mathcal{P}(t) \sim 1$, indicating that all probability is concentrated in the initial level all the time. An alternative way to identify this regime is from $\delta E_{\text {core }}(t)$ which is trivially equal to $\Delta$. 
- Extended perturbative regime: The appearance of a core-tail structure which is characterized by a separation of scales $\Delta \ll \delta E_{\text {core }}(t) \ll \delta E(t) \ll \Delta_{b}$. The core is of non-perturbative nature, but the variance $\delta E^{2}(t)$ is still dominated by the tails. The latter are described by perturbation theory.

- Non-perturbative regime: The existence of this regime is associated with having the finite energy scale $\Delta_{b}$. It is characterized by $\Delta_{b} \ll \delta E_{\text {core }}(t) \sim \delta E(t)$. As implied by the terminology, perturbation theory (to any order) is not a valid tool for the analysis of the energy spreading. Note that in this regime, the spreading profile is characterized by a single energy scale $\left(\delta E \sim \delta E_{\text {core }}\right)$.

\subsubsection{The energy spreading $\delta E(t)$}

Of special importance for understanding quantum dissipation is the theory for the variance $\delta E^{2}(t)$ of the energy spreading. Having $\delta E(t) \propto \delta k$ means linear response. If $\delta E(t) / \delta k$ depends on $\delta k$, we call it "nonlinear response". In this paragraph we explain that linear response theory (LRT) is based on the "LRT formula" Eq. (4.9) for the spreading. This formula has a simple classical derivation (see Subsection 4.2.1.1 below).

It is understood that we always assume the classical conditions for the validity of Eq. (4.9) to be satisfied (no $\hbar$ involved in such conditions). The question is what happens to the validity of LRT once we quantize the system $[58,59,138,60,112]$.

The immediate (naive) tendency is to regard LRT as the outcome of quantum mechanical first order perturbation theory (FOPT). In fact, the regimes of validity of FOPT and LRT do not coincide. On the one hand we have the adiabatic regime where FOPT is valid as a leading order description, but not for response calculation. On the other hand, the validity of Eq. (4.9) goes well beyond FOPT. This leads to the (correct) identification [52, 58, 60] of what we call the "perturbative regime". The border of this regime is determined by the energy scale $\Delta_{b}$, while $\Delta$ is not involved. Outside of the perturbative regime we cannot trust the LRT formula. However, as we further explain below, the fact that Eq. (4.9) is not valid in the non-perturbative regime does not imply that it fails there.

We stress again that one should distinguish between "non-perturbative response" and "nonlinear response". These are not synonyms. As we explain in the next paragraph, the adiabatic regime is "perturbative" but "nonlinear", while the semiclassical limit is "nonperturbative" but "linear".

In the adiabatic regime, FOPT implies zero probability to make a transitions to other levels. Therefore, to the extent that we can trust the adiabatic approximation, all probability remains concentrated on the initial level. Thus, in the adiabatic regime Eq. (4.9) is not a valid formula: It is essential to use higher orders of perturbation theory, and possibly non-perturbative corrections (Landau-Zener [210, 211]), in order to calculate the response. Still, FOPT provides a meaningful leading order description of the dynamics (i.e. having no transitions), and therefore we do not regard the adiabatic nonlinear regime as "nonperturbative". 
In the non-perturbative regime the evolution of $P_{t}(n \mid m)$ cannot be extracted from perturbation theory, not in leading order nor in any order. Still this does not necessarily imply a nonlinear response. On the contrary, the semiclassical limit is contained in the deep nonperturbative regime $[58,60,112,115]$. There, the LRT formula Eq. (4.9) is in fact valid. But its validity is not a consequence of perturbation theory, but rather the consequence of quantum-classical correspondence (QCC).

In the next subsection we will present a classical derivation of the general LRT expression (4.9). In Subsection 4.2.1.2 we derive it using first order perturbation theory (FOPT). In Subsection 4.2.2 we derive the corresponding FOPT expression for the survival probability.

\subsubsection{Classical LRT derivation for $\delta E(t)$}

The classical evolution of $E(t)=\mathcal{H}(I(t), \varphi(t))$ can be derived from Hamilton's equations. Namely,

$$
\frac{\mathrm{d} E(t)}{\mathrm{d} t}=[\mathcal{H}, \mathcal{H}]_{\mathrm{PB}}+\frac{\partial \mathcal{H}}{\partial t}=-\delta k \dot{f}(t) \mathcal{F}(t),
$$

where $[\cdot]_{\mathrm{PB}}$ indicates the Poisson brackets and $\mathcal{F}(t)$ is the generalized force introduced in Subsection 3.1.2. Integration of Eq. (4.12) leads to

$$
E(t)-E(0)=-\delta k \int_{0}^{t} \mathcal{F}\left(t^{\prime}\right) \dot{f}\left(t^{\prime}\right) d t^{\prime} .
$$

Taking a micro-canonical average over initial conditions we obtain the following expression for the variance

$$
\delta E^{2}(t)=\delta k^{2} \int_{0}^{t} C\left(t^{\prime}-t^{\prime \prime}\right) \dot{f}\left(t^{\prime}\right) \dot{f}\left(t^{\prime \prime}\right) d t^{\prime} d t^{\prime \prime},
$$

which can be re-written in the form of (4.9). Here $C\left(t^{\prime}-t^{\prime \prime}\right)$ is the autocorrelation function of the generalized force $\mathcal{F}(t)$ (see Sec. 3.1.2).

An extreme case of Eq. (4.9) is the sudden limit for which $f(t)$ is a step function. Such an evolution is equivalent to the LDoS studies of Chapter 3. In this case $F_{t}(\omega)=1$, and accordingly we recover the result from (3.32), namely

$$
\delta E_{\mathrm{cl}}=\delta k \times \sqrt{C(0)} \quad \text { ["sudden" case] . }
$$

Another special case is the response for persistent (either linear or periodic) driving of a system with an extremely short correlation time. In such a case $F_{t}(\omega)$ becomes a narrow function with a weight that grows linearly in time. For linear driving $(f(t)=t)$ we get $F_{t}(\omega)=t \times 2 \pi \delta(\omega)$. This implies diffusive behavior:

$$
\delta E(t)=\sqrt{2 D_{E} t} \quad \text { ["Kubo" case], }
$$

where $D_{E} \propto \delta k^{2}$ is the diffusion coefficient. The expression for $D_{E}$ as an integral over the correlation function is known in the corresponding literature either as Kubo formula, or as Einstein relation, and is the cornerstone of the Fluctuation-Dissipation relation. 


\subsubsection{Quantum LRT derivation for $\delta E(t)$}

The quantum mechanical derivation looks like an exercise in first order perturbation theory. In fact a proper derivation that extends and clarifies the regime where the result is applicable requires infinite order. If we want to keep a complete analogy with the classical derivation we should work in the adiabatic basis [52]. (For a brief derivation see Appendix D of Ref. [214]).

In the following presentation we work in a "fixed basis" and assume $f(t)=f(0)=0$. We use the standard textbook FOPT expression for the transition probability from an initial state $m$ to any other state $n$. This is followed by integration by parts. Namely,

$$
\begin{aligned}
P_{t}(n \mid m) & =\frac{\delta k^{2}}{\hbar^{2}}\left|\mathbf{B}_{n m}\right|^{2}\left|\int_{0}^{t} \mathrm{~d} t^{\prime} f\left(t^{\prime}\right) e^{i\left(E_{n}-E_{m}\right) t^{\prime} / \hbar}\right|^{2} \\
& =\frac{\delta k^{2}}{\hbar^{2}}\left|\mathbf{B}_{n m}\right|^{2} \frac{\tilde{F}_{t}\left(\omega_{n m}\right)}{\left(\omega_{n m}\right)^{2}},
\end{aligned}
$$

where $\omega_{n m}=\left(E_{n}-E_{m}\right) / \hbar$. Now we calculate the variance and use Eq. (3.14) so as to get

$$
\begin{aligned}
\delta E^{2}(t) & =\sum_{n} P_{t}(n \mid m)\left(E_{n}-E_{m}\right)^{2} \\
& =\delta k^{2} \int_{-\infty}^{\infty} \frac{\mathrm{d} \omega}{2 \pi} \tilde{C}(\omega) \tilde{F}_{t}(\omega) .
\end{aligned}
$$

\subsubsection{Restricted QCC}

As already seen in Section 3.5 of the LDoS studies, the FOPT result for the quantum mechanical energy spreading $\delta E(t)$ is exactly the same as the classical expression Eq. (4.9). Since this quantum-classical correspondence applies only for the second moment of the energy distribution it was termed "restricted QCC". We recall from the discussion in Section 3.5 that this very robust correspondence [60] should be contrasted with "detailed QCC" that applies only in the semiclassical regime where $P_{t}(n \mid m)$ can be approximated by a classical result $P_{t}^{\mathrm{cl}}(n \mid m)$ (and not by a perturbative result). For the definition of the classical profile $P_{t}^{\mathrm{cl}}(n \mid m)$ in the wavepacket dynamics scenario see beginning of Section 4.3.

\subsubsection{Quantum LRT derivation for $\mathcal{P}(t)$}

With the validity of FOPT assumed, we can also calculate the time-decay of the survival probability $\mathcal{P}(t)$. From Eq. (4.17) we get:

$$
p(t) \equiv \sum_{n\left(\neq n_{0}\right)} P_{t}(n \mid m)=\delta k^{2} \int_{-\infty}^{\infty} \frac{d \omega}{2 \pi} \tilde{C}(\omega) \frac{\tilde{F}_{t}(\omega)}{(\hbar \omega)^{2}} .
$$


Assuming that $\mathcal{P}(t)=1-p(t)$ can be extrapolated in a "stochastic" fashion we get Eq. (4.10). Another way to write the final formula is as follows:

$$
\mathcal{P}(t)=\exp \left[-\frac{1}{\hbar^{2}} \int_{0}^{t} \int_{0}^{t} C\left(t^{\prime}-t^{\prime \prime}\right) \delta x\left(t^{\prime}\right) \delta x\left(t^{\prime \prime}\right) d t^{\prime} d t^{\prime \prime}\right] .
$$

For constant perturbation (wavepacket dynamics) and assuming long times we obtain (see also Sec. 4.3.3) the Wigner decay

$$
\mathcal{P}(t)=\exp \left[-\left(\frac{\delta k}{\hbar}\right)^{2} \tilde{C}(\omega=0) \times t\right],
$$

which can be regarded as a special case of Fermi's golden rule.

\subsubsection{The survival probability and the LDoS}

For constant perturbation it is useful to remember that $\mathcal{P}(t)$ is connected to the LDoS as follows:

$$
\begin{aligned}
\mathcal{P}(t) & \equiv\left|\left\langle n\left(k_{0}\right)\left|e^{-i \hat{H}(k) t / \hbar}\right| n\left(k_{0}\right)\right\rangle\right|^{2}=\left.\left.\left|\sum_{m} e^{-i E_{m}(k) t / \hbar}\right|\left\langle m(k) \mid n\left(k_{0}\right)\right\rangle\right|^{2}\right|^{2} \\
& =\left|\int_{\infty}^{\infty} P(E \mid m) e^{-i E t / \hbar} d E\right|^{2},
\end{aligned}
$$

i.e., the survival probability can be written in term of the Fourier transform of the LDoS. This implies that a Lorentzian approximation for the LDoS will lead to an exponential decay of the survival probability. In the non-perturbative regime the LDoS is not a Lorentzian, and therefore one should not expect an exponential. In the semiclassical regime the LDoS shows system specific features and therefore the decay of $\mathcal{P}(t)$ becomes non-universal.

\subsection{Wavepacket dynamics of cold bosons on an optical lattice}

After discussing in the previous section the general linear response theory for driven systems we are now focusing on the wavepacket dynamics [116] in the framework of cold bosons on optical lattices. We consider an initial preparation which is high enough in energy such that the corresponding underlying classical dynamics is chaotic. Will the results follow the above depicted scenario of the Wigner model or will the interatomic interactions change the picture? An overview of the regimes of $P_{t}(r)$ is given in Fig. 4.2 where we plot 
a)

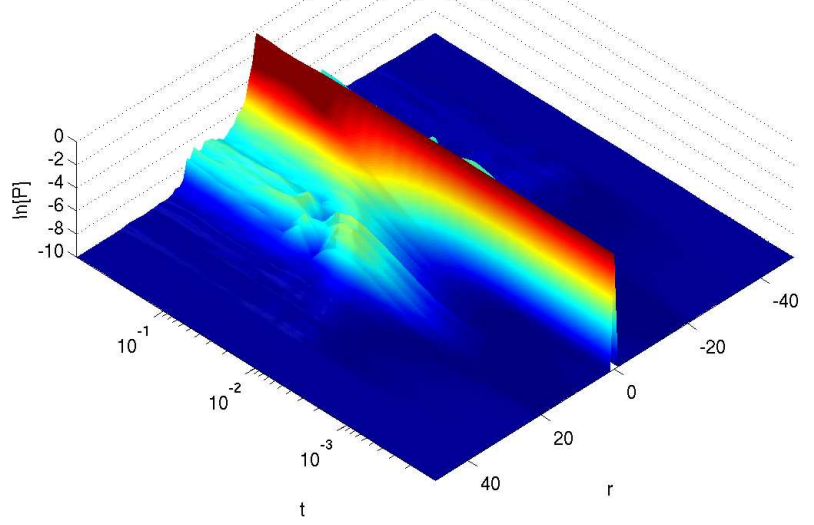

b)

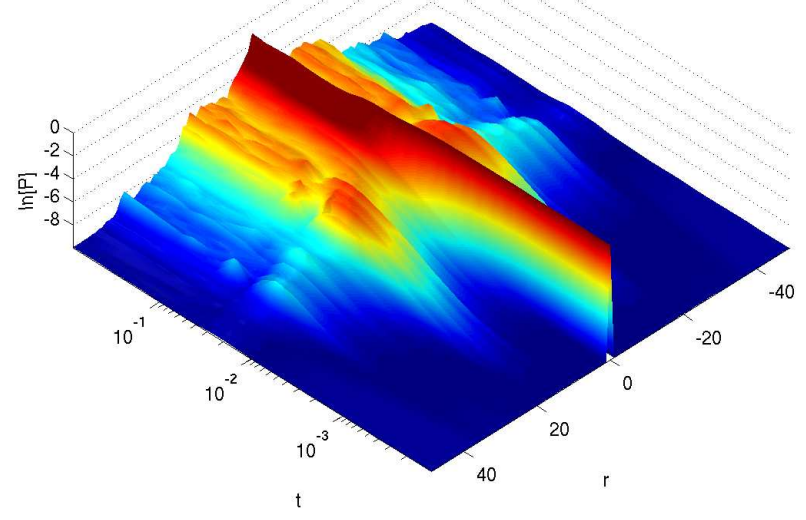

c)

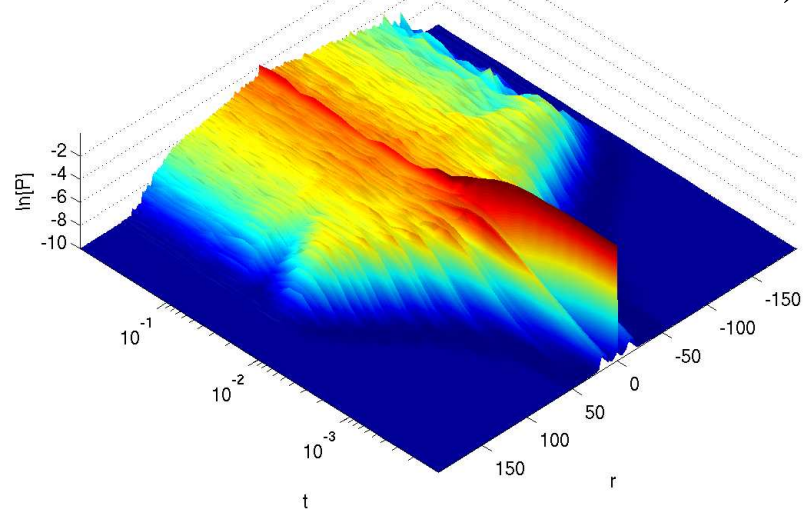

Figure 4.2.: The profile $P_{t}(r)$ of the BHH plotted as a function of time for various perturbation strengths $\delta k<\delta k_{\mathrm{qm}}$ (a), $\delta k_{\mathrm{qm}}<\delta k<\delta k_{\mathrm{prt}}$ (b), $\delta k>\delta k_{\mathrm{prt}}$ (c). Note the different scale in (c). Here, $N=70, \tilde{E}=0.26$ and $\lambda_{0}=0.053$. 
the quantum spreading profile as a function of time $t$ for three different perturbation strengths $\delta k$. Already from there we recognize a different behavior than in the Wigner model, namely during the evolution, pronounced sidebands emerge. While these sidebands are familiar from the LDoS studies in the previous chapter, we furthermore observe an unexpected "squeezing" of the profile at $t \approx 0.02$. The latter is a consequence of the lattices dynamics and will lead to a drastic effect in the study of quantum stability which is the topic of the next chapter.

We start the discussion with the classical dynamics and then turn to the evolution of the quantum profile $P_{t}(r)$ in the various regimes which we describe in terms of the energy spreading $\delta E(t)$ (Subsection 4.3.2) and the survival probability $\mathcal{P}(t)$ (Subsection 4.3.3). Our findings on the detailed quantum-classical correspondence are reported in Subsection 4.3.4. After that we investigate the energy spreading of the IRMT model with focus on the non-perturbative regime $\delta k>\delta k_{\text {prt }}$ where we find a qualitatively different behavior from the dynamical BHH model. Finally, we report the temporal evolution of the quantum occupation number $n_{i}(t)$ for the trimer.

\subsubsection{Classical dynamics}

The classical picture is quite clear $[57,137,112,116]$ : The initial preparation is assumed to be a micro-canonical distribution that is supported by the energy surface $\mathcal{H}_{0}(I, \varphi)=$ $E(0)=E_{n_{0}}$ where the Hamiltonian is given by Eq. (2.23) with $f=3$ and $v_{i}=0$. Taking $\mathcal{H}=\mathcal{H}_{0}-\delta k \mathcal{B}$ to be the generator of the classical dynamics, the phase-space distribution spreads away from the initial surface for $t>0$. "Points" of the evolving distribution move upon the energy surfaces of $\mathcal{H}(I, \varphi)$. Thus, the energy $E(t)=\mathcal{H}_{0}(I(t), \varphi(t))$ of the evolving distribution spreads with time.

Similarly to the LDoS case in Section 3.5 we can now define a classical spreading profile $P_{t}^{\mathrm{cl}}\left(n \mid n_{0}\right)$ which we expect to match the quantum $P_{t}\left(n \mid n_{0}\right)$ in the deep non-perturbative, i.e. semiclassical, regime of the BHH. To this end we first propagate a large set of trajectories $\{E\}_{t=0}$ that originate from the energy surface $\mathcal{H}_{0}(I, \varphi)=E(t=0)=E_{n_{0}}$ under the Hamiltonian $\mathcal{H}$ up to time $t$. Projecting them back ${ }^{4}$ onto $\mathcal{H}_{0}$ yields a set of energies $\{E\}_{t=t}$ whose distribution ${ }^{5}$ constitutes the spreading profile $P_{t}^{\mathrm{cl}}\left(n \mid n_{0}\right)$ at the time $t$. In complete analogy to the quantum case, the classical survival probability $\mathcal{P}_{\mathrm{cl}}(t)$ is the projection of the spreading profile on the initial excitation energy, i.e. $\mathcal{P}_{\mathrm{cl}}(t)=P_{t}^{\mathrm{cl}}\left(n_{0} \mid n_{0}\right)$.

The classical energy spreading $\delta E_{\mathrm{cl}}(t)$ can be evaluated using the LRT formula Eq. (4.11) with a rectangular pulse $f\left(t^{\prime}\right)=1$ for $0<t^{\prime}<t$ (see also Fig. 4.1). We get

$$
\tilde{F}_{t}(\omega)=\left|1-e^{-i \omega t}\right|^{2}=(\omega t)^{2} \operatorname{sinc}^{2}\left(\frac{\omega t}{2}\right)
$$

\footnotetext{
${ }^{4}$ Note that the quantum profile $P_{t}(n \mid m)$ corresponds to projecting the time-evolved state $\hat{U}(t)\left|n_{0}\right\rangle$ onto the initial basis $\left\{\left|n_{0}\right\rangle\right\}$.

${ }^{5}$ Technically, this requires calculating the histogram with a bin-size given by the mean level spacing $\Delta$.
} 


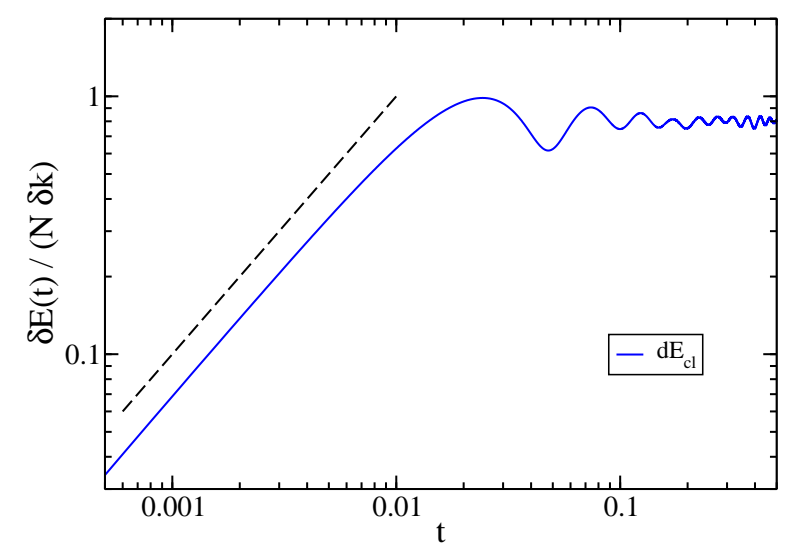

Figure 4.3.: The classical energy spreading $\delta E_{\mathrm{cl}}(t)$ for the $\mathrm{BHH}$ (normalized with respect to the perturbation strength $\delta k$ and the boson number $N$ ) is plotted as a function of time. The dashed line has slope one and is drawn to guide the eye. The agreement with the ballistic spreading followed by saturation as predicted by Eq. (4.25) is apparent.

and hence

$$
\delta E_{\mathrm{cl}}(t)=\delta k \times \sqrt{2(C(0)-C(t))} .
$$

For times shorter than the classical correlation time $t \ll \tau_{\text {cl }}$ (see Eq. (3.13)) we can expand the correlation function as $C(t) \approx C(0)-\frac{1}{2} C^{\prime \prime}(0) t^{2}$, leading to a ballistic evolution. Then, for $t \gg \tau_{\mathrm{cl}}$, due to ergodicity a "steady-state distribution" appears, where the evolving "points" occupy an energy shell in phase-space. The thickness of this energy shell equals $\delta E_{\mathrm{cl}}$. Thus, we have a crossover from ballistic energy spreading to saturation:

$$
\delta E_{\mathrm{cl}}(t) \approx\left\{\begin{array}{ll}
\sqrt{2}\left(\delta E_{\mathrm{cl}} / \tau_{\mathrm{cl}}\right) t & \text { for } t<\tau_{\mathrm{cl}} \\
\sqrt{2} \delta E_{\mathrm{cl}} & \text { for } t>\tau_{\mathrm{cl}}
\end{array} .\right.
$$

Figure 4.3 shows the scaled classical energy spreading $\delta E_{\mathrm{cl}}(t) /(N \delta k)$ for the $\mathrm{BHH}$. The heavy dashed line has slope one and is drawn to guide the eye. In Subsection 3.1.2 we found $\tilde{\tau}_{\mathrm{cl}}=\tau_{\mathrm{cl}} \tilde{U} \approx 2 \pi$ leading to $\tau_{\mathrm{cl}} \approx 0.02$ for the parameters used here. In agreement with Eq. (4.25) we see that $\delta E_{\mathrm{cl}}(t)$ is first ballistic and then saturates at $\tau_{\mathrm{cl}}$. Hence, the classical dynamics is fully characterized by the two classical parameters $\tau_{\mathrm{cl}}$ and $\delta E_{\mathrm{cl}}$.

\subsubsection{Quantum energy spreading}

Let us now look at the quantum Bose-Hubbard Hamiltonian. An overview of the spreading profile $P_{t}(r)$ for three representative perturbation strengths $\delta k$ is given in Fig. 4.2. We find qualitative differences in the spreading depending on the value of $\delta k$, which we discuss in the following, starting with the perturbative regimes.

For small perturbations $\delta k<\delta k_{\mathrm{qm}}$ (see Fig. 4.2a) the probability is mainly concentrated in the initial level during the entire evolution. This is the FOPT regime where the pertur- 

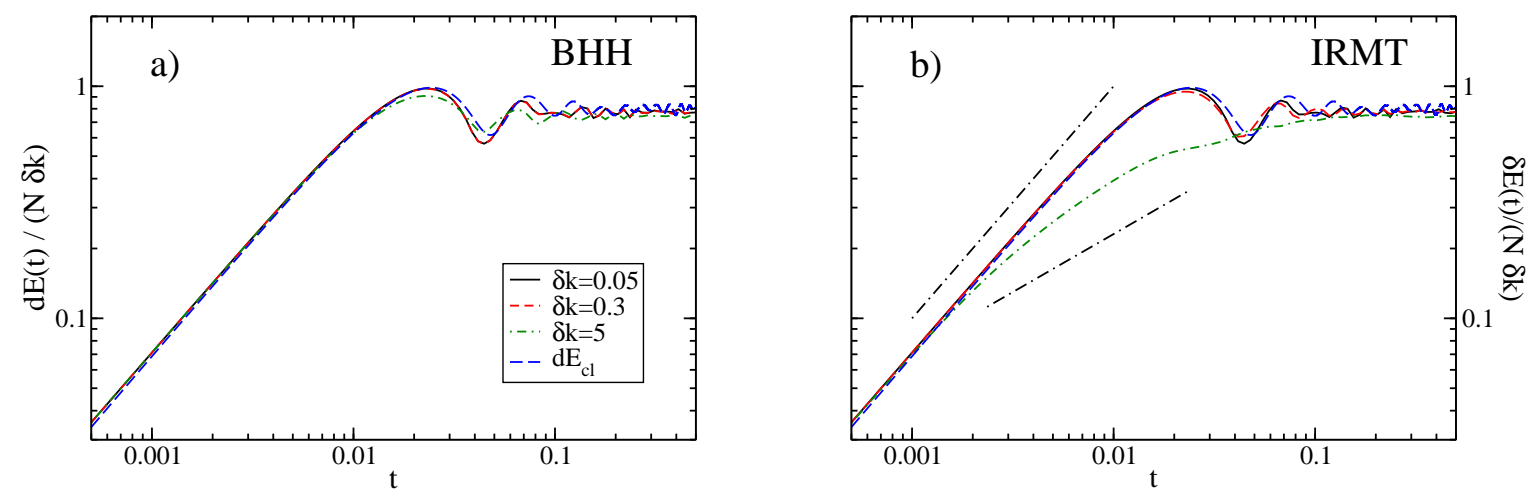

Figure 4.4.: The (normalized) energy spreading $\delta E(t)$ for the BHH (a) and the IRMT model (b) for three different perturbation strengths $\delta k=0.05<\delta k_{\mathrm{qm}}$ (solid black line), $\delta k_{\mathrm{qm}}<\delta k=0.3<\delta k_{\mathrm{prt}}$ (dashed red line), and $\delta k=5>\delta k_{\text {prt }}$ (dash-dotted green line). The classical expectation $\delta E_{\mathrm{cl}}(t)$ is represented in both plots by a dashed blue line for comparison. In the right panel the black dashdotted lines have slope one and one-half respectively and are drawn to guide the eye. While for the BHH model one observes restricted quantum classical correspondence in all regimes this is not the case for the IRMT model (b): For perturbations $\delta k>\delta k_{\text {prt }}$ the energy spreading $\delta E(t)$ exhibits a premature crossover to diffusive behavior. Here, $N=230, \tilde{U}=280, \tilde{E}=0.26$ and $\lambda_{0}=0.053$.

bation strength mixes only nearby levels and little probability escapes to the tails. ${ }^{6}$ At the same time one observes the appearance of sidebands in the profile. Using our experience from the LDoS study we can readily identify them as a consequence of the pronounced structure in the bandprofile of the perturbation matrix B (see Fig. 3.7). In Subsection 3.1.2 the position of the main peaks in $\tilde{C}(\omega)$ was determined to be around a frequency $\omega \approx 150$ which corresponds to $r=\omega \hbar / \Delta \approx 23$ and is in agreement with the data reported in Fig. 4.2. We note that the bandwidth $\Delta_{b} \approx \tilde{U}$ corresponds to $b=\Delta_{b} / \Delta \approx 44$.

As the perturbation strength is increased $\delta k_{\mathrm{qm}}<\delta k<\delta k_{\mathrm{prt}}$ (Fig. 4.2b), levels within the bandwidth are mixed and one can distinguish two different components in the profile $P_{t}(r)$. These are the core, where most of the probability is concentrated (characterized by $\delta E_{\text {core }}(t)$ ), and the tail component (characterized by $\delta E(t)$ ). The latter is reported in Fig. 4.4a together with the classical spreading $\delta E_{\mathrm{cl}}(t) .{ }^{7}$ The remarkable fact is that, as far as $\delta E(t)$ is concerned, the agreement with the classical result is perfect. This is the above discussed restricted QCC which could lead to the wrong impression that the classical and quantum spreading are of the same nature. However, this is definitely not the case.

In order to detect this different nature of the quantum ballistic-like spreading we have to inquire a measure that is sensitive to the structure of the profile like the core-width $\delta E_{\text {core }}(t)$. If the spreading were of classical type this would imply that the spreading profile would grow homogeneously as schematically depicted in the right panel of Fig. 4.5. Hence

\footnotetext{
${ }^{6}$ The rate is given by FOPT to be proportional to $t^{2}$, see left panel of Fig. 4.5.

${ }^{7}$ In the perturbative regime $\delta k<\delta k_{\text {prt }}$, the IRMT model gives the same results as the BHH. Thus, we will use IRMT and LRT as synonyms for $\delta k<\delta k_{\text {prt. }}$ See also around Eq. (4.11).
} 

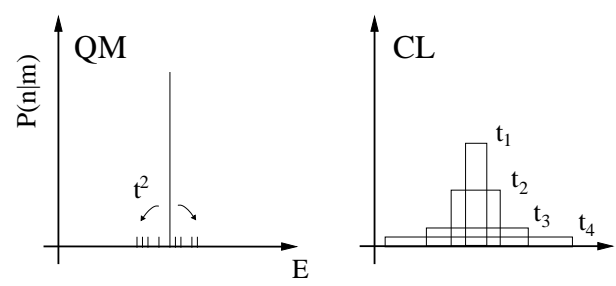

Figure 4.5.: Scheme of the FOPT quantum and the classical spreading of the profile $P_{t}(m \mid n)$. Although they are of different nature they lead to the same ballistic behavior of the variance.

it would be characterized by a single energy scale, i.e. $\delta E(t) \sim \delta E_{\text {core }}(t)$. That this is not the case can be seen by looking at Fig. 4.6a where we plot $\delta E_{\text {core }}(t)$. While in the standard perturbative regime only one level is involved, the core width $\delta E_{\text {core }}(t)$ grows with increasing perturbation strength $\delta k$ (but the tails are still captured by FOPT). We stress the fact that as long as we have a separation of energy scales, i.e. $\delta E_{\text {core }}(t)<\delta E(t)<\Delta_{b}$, we can still use (infinite order) perturbation theory. Once we enter the non-perturbative regime, $\delta k>\delta k_{\text {prt }}$ the core spills over the bandwidth $\Delta_{b}<\delta E(t) \sim \delta E_{\text {core }}(t)$ and the separation of scales is lost. This can be nicely seen in Fig. 4.2c (mind the different scale) where also higher order sidebands evolve.

Finally, let us compare the evolution of the core-width with the participation ratio $\delta n_{\text {IPR }}$. This quantity is a measure for the number of contributing components of a wavefunction. Accordingly, if the profile is very homogeneous we would expect that the number of contributing levels equals the number of states that constitute the core, i.e., $\delta n_{\mathrm{IPR}}(t) \times \Delta \sim$ $\delta E_{\text {core }}(t)$. If, however, the profile is very sparse we would expect $\delta n_{\text {IPR }}(t) \times \Delta \ll \delta E_{\text {core }}(t)$. In Fig. 4.6b we report the scaled participation ratio $\delta n_{\mathrm{IPR}}(t) \Delta /(N \delta k)$. One can observe that it fairly resembles the core width $\delta E_{\text {core }}(t)$. These deviations can be attributed to the fact that the shape of the profile is not constant but structured, hence the participation ratio $\delta n_{\text {IPR }}$ underestimates the number of contributing levels.

\subsubsection{The survival probability $\mathcal{P}(t)$}

In order to differentiate between short- and long-time decay in the survival probability, it is useful to rewrite the quantum LRT formula for $\mathcal{P}(t)$ using (4.23) as

$$
\mathcal{P}(t)=\exp \left[-\left(\frac{\delta k}{\hbar}\right)^{2} \times \int_{-\infty}^{\infty} \frac{d \omega}{2 \pi} \tilde{C}(\omega) t t \operatorname{sinc}^{2}\left(\frac{\omega t}{2}\right)\right] .
$$

For short times $\left(t \ll \tau_{\mathrm{cl}}\right)$ during which the spreading is ballistic-like, the term $t \operatorname{sinc}^{2}(\omega t / 2)$ is broad compared to the bandprofile and can be approximated by $t$ leading to

$$
\mathcal{P}(t)=\exp \left[-C(\tau=0) \times\left(\frac{\delta k t}{\hbar}\right)^{2}\right] .
$$

For longer times $\left(t \gg \tau_{\mathrm{cl}}\right)$ on the other hand, the term $t \operatorname{sinc}^{2}(\omega t / 2)$ is extremely narrow and can be approximated by a delta function $\delta(\omega)$. This results in the FGR decay of Eq. (4.21). 

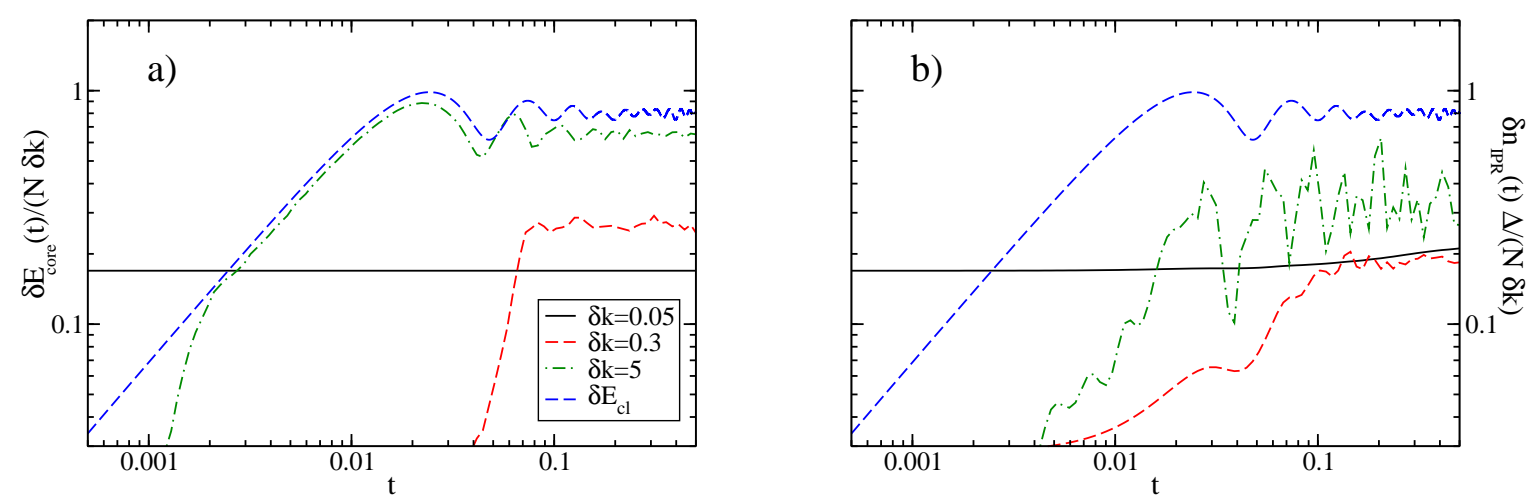

Figure 4.6.: Simulations of the wavepacket dynamics for the BHH model for various perturbation strengths and the same parameters as in Fig. 4.4. The classical spreading $\delta E_{\mathrm{cl}}(t)$ is represented in both plots by a blue dashed line for the sake of comparison. The evolution (a) of the corresponding core width $\delta E_{\text {core }}(t)$ is plotted as a function of time. In the perturbative regimes one observes a separation of scales $\delta E_{\text {core }}(t)<\delta E(t)<\Delta_{b}$, which is lost for strong perturbations $\delta k>\delta k_{\text {prt }}$, where $\delta E_{\text {core }}(t)$ approaches more and more the classical expectation $\delta E_{\mathrm{cl}}(t)$. In panel (b) we plot the the corresponding scaled participation ratio $\delta n_{\mathrm{IPR}}(t) \Delta /(N \delta k)$ which fairly resembles the behavior of the core width $\delta E_{\text {core }}(t)$. See text for details.

How much can we trust these expressions? Obviously FOPT can be trusted as long as $\mathcal{P}(t) \sim 1$. This can be converted into an inequality $t<t_{\text {prt }}$ where

$$
t_{\mathrm{prt}}=\left(\frac{\delta k_{\mathrm{prt}}}{\delta k}\right)^{\mathrm{v}=1,2} \tau_{\mathrm{cl}} .
$$

The power $v=1$ applies to the non-perturbative regime where the breakdown of $\mathcal{P}(t)$ happens to be before $\tau_{\mathrm{cl}}$. The power $\mathrm{v}=2$ applies to the perturbative regime where the breakdown of $\mathcal{P}(t)$ happens after $\tau_{\mathrm{cl}}$ at $t_{\mathrm{prt}}=\hbar / \Gamma$, i.e. after the ballistic-like stage. In Fig. 4.7 we present the decay of $\mathcal{P}(t)$ for the three regimes. We superimpose the data obtained from the IRMT model introduced in Section 3.2 which represents the quantum LRT (see also below Eq. (4.11)). In both perturbative regimes we observe a short initial Gaussian decay (as implied by Eq. (4.27)) which is followed by the exponential FGR decay. In the FOPT regime (inset of Fig. 4.7a) the entire decay until saturation is described by LRT. In the extended perturbative regime (see Fig. 4.7a) the overall agreement is still pretty good. However, here the perturbative break time $t_{\mathrm{prt}}$ is shorter and one finds a deviation around the time $t_{\mathrm{prt}} \sim 0.01$ calculated from (4.28).

For stronger perturbations $\delta k>\delta k_{\text {prt }}$ LRT breaks down after the initial Gaussian decay (4.27) around the calculated break time $t_{\mathrm{prt}} \sim 0.001$. The long term behavior of $\mathcal{P}(t)$ in the non-perturbative regime is not the Wigner decay. It can be obtained by Fourier transform of the LDoS (see Subsection 4.2.3) which we superimpose in Fig. 4.7b as blue circles. The agreement with $\mathcal{P}(t)$ is excellent.

How do the quantum results compare to $\mathcal{P}_{\mathrm{cl}}(t)$ in the non-perturbative regime $\delta k>\delta k_{\mathrm{prt}}$ ? 

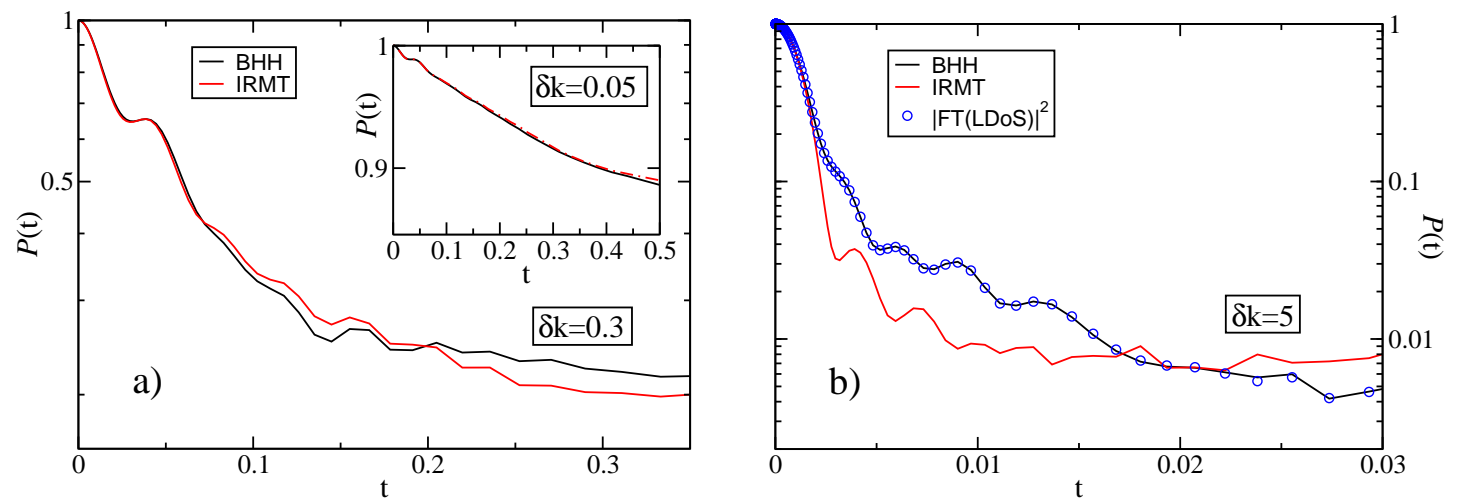

Figure 4.7.: The survival probability $\mathcal{P}(t)$ for the BHH and three different perturbation strengths a) $\delta k=0.05<\delta k_{\mathrm{qm}}$ (inset), $\delta k_{\mathrm{qm}}<\delta k=0.3<\delta k_{\mathrm{prt}}$ (main figure), and b) $\delta k=5>\delta k_{\mathrm{prt}}$. The solid black lines represent the exact numerical result while the solid red lines are the LRT result (4.10) calculated using the IRMT model (see also Footnote 7). The inset of panel (a) represents the FOPT regime, while the main figure corresponds to the extended perturbative regime. Here the break time is $t_{\text {prt }} \sim 0.1$ given by Eq. (4.28). In the non-perturbative regime (b), the LRT breaks down close to the calculated break time $t_{\mathrm{prt}} \sim 0.001$. In this panel we superimpose the Fourier transform of the LDoS as blue circles. The agreement with $\mathcal{P}(t)$ is excellent. Here, $N=230, \tilde{U}=280, \tilde{E}=0.26$ and $\lambda_{0}=0.053$.

In Fig. 4.8 we report the classical $\mathcal{P}_{\mathrm{cl}}(t)$ vs. the quantum $\mathcal{P}(t)$. Both decays follow a powerlaw. Specifically, we find for the quantum decay

$$
\mathcal{P}(t) \sim t^{-3 / 2}
$$

while the classical survival probability decays as

$$
\mathcal{P}_{\mathrm{cl}}(t) \sim t^{-1} \text {. }
$$

Although the quantum decay rate is larger than the classical one, the overall quantum decay is slower due to interference phenomena. Naively, one might have expected stronger developed QCC in the deep non-perturbative regime. We will use the next subsection to determine in how far these deviations influence other moments of the distribution $P_{t}(r)$.

Surprisingly, close to the classical correlation time $\tau_{\mathrm{cl}} \sim 0.02$ the survival probability $\mathcal{P}(t)$ displays a sequence of peaks which coincides with the dips in the spreading $\delta E(t)$ (see Fig. 4.4a). This is the "squeezing" of the profile that can be seen from Fig. 4.2. This phenomenon will play a prominent role in the next chapter. At this point we only mark, that it is not a trivial recurrence associated with the discreteness of the quantum system but rather related to the lattice dynamics.

\subsubsection{Detailed quantum-classical correspondence}

In Subsection 4.3.2 we have seen that the Bose-Hubbard Hamiltonian exhibits restricted quantum-classical correspondence. Does it also show detailed QCC [52, 137, 112], i.e., 


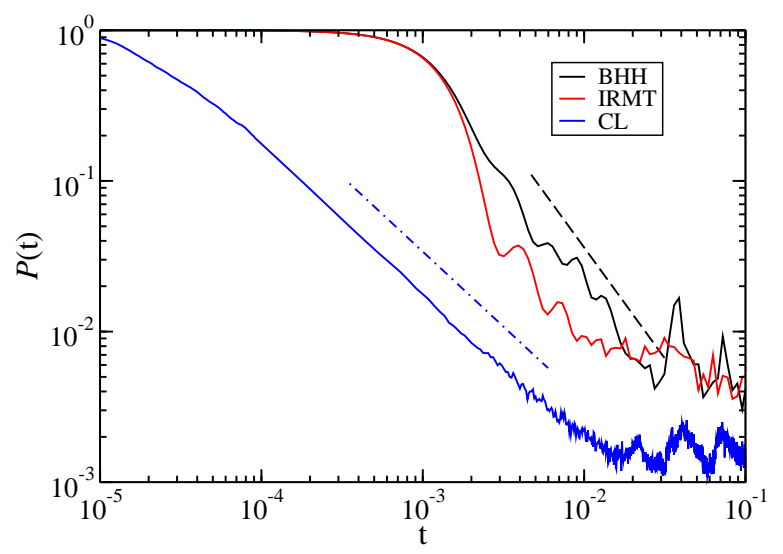

Figure 4.8.: The quantum and classical survival probability $\mathcal{P}(t)$ in the non-perturbative regime $\delta k=5>\delta k_{\mathrm{prt}}$. The IRMT result is overplotted as a red line for comparison. The straight lines represent best fits and have negative slope 1.47 (dashed black line) and 1 (dash-dotted blue line) respectively. Here, $N=230, \tilde{U}=280, \tilde{E}=0.26$ and $\lambda_{0}=0.053$.

can we describe the quantum spreading profile $P_{t}\left(n \mid n_{0}\right)$ by the classical one $P_{t}^{\mathrm{cl}}\left(n \mid n_{0}\right)$ ? From the previous discussion it should be clear that we must not expect detailed QCC in the perturbative regimes. In fact, this can be also seen by analyzing the saturation profile $P_{\infty}(n \mid m)$ which we plot in Fig. 4.9 for the BHH and the IRMT model in the three regimes. Analytically, $P_{\infty}(n \mid m)$ is obtained from the diagonal approximation ${ }^{8}$ of the spreading profile for large times:

$$
P_{\infty}(n \mid m)=\sum_{n^{\prime}}\left|\left\langle n\left(k_{0}\right) \mid n^{\prime}(k)\right\rangle\right|^{2}\left|\left\langle n^{\prime}(k) \mid m\left(k_{0}\right)\right\rangle\right|^{2},
$$

and can be regarded as the autoconvolution of $P(n \mid m)=|\langle n(k) \mid m(0)\rangle|^{2}$. Thus the average saturation profile $P_{\infty}(r)$ is approximately related to the average shape of eigenstates/LDoS. From the previous chapter we know that the perturbative LDoS profiles are characterized by genuine quantum energy scales (like $\Delta, \Gamma, \Delta_{b}$ ) while the classical ergodic distribution is characterized by the single energy scale $\delta E_{\mathrm{cl}}$. Looking at Figs. $4.2 \mathrm{c}, 4.4 \mathrm{~b}$ we confirm that for strong perturbations $\delta k>\delta k_{\text {prt }}$ (and after a short quantal transition period) there is no scale separation $\left(\delta E_{\text {core }}, \Delta_{b}\right)$. Thus we are left with the non-perturbative regime as the only candidate to observe detailed quantum classical correspondence.

In Fig. 4.10 we plot four snapshots of the classical and quantum evolving profiles in this regime. After the quantal transition period (from Fig. 4.4b we extracted $t \sim 0.002$ ) we observe a reasonable agreement between the classical and the quantum profile, i.e. detailed QCC [52, 137, 112]. However, this agreement does not include the survival probability $\mathcal{P}(t)$, since the evolving component $P_{t}\left(n_{0} \mid n_{0}\right)=\mathcal{P}(t)$ associated with the initial excitation $\left|n_{0}\right\rangle$ decays slower than the classical $\mathcal{P}_{\mathrm{cl}}(t)$ as discussed in the previous subsection (see Fig. 4.8). Nevertheless, note that the initial (quantum dictated) decay is well captured by the

\footnotetext{
${ }^{8}$ For large times $t$ the oscillating terms in (4.31) are assumed to average out and can be neglected.
} 


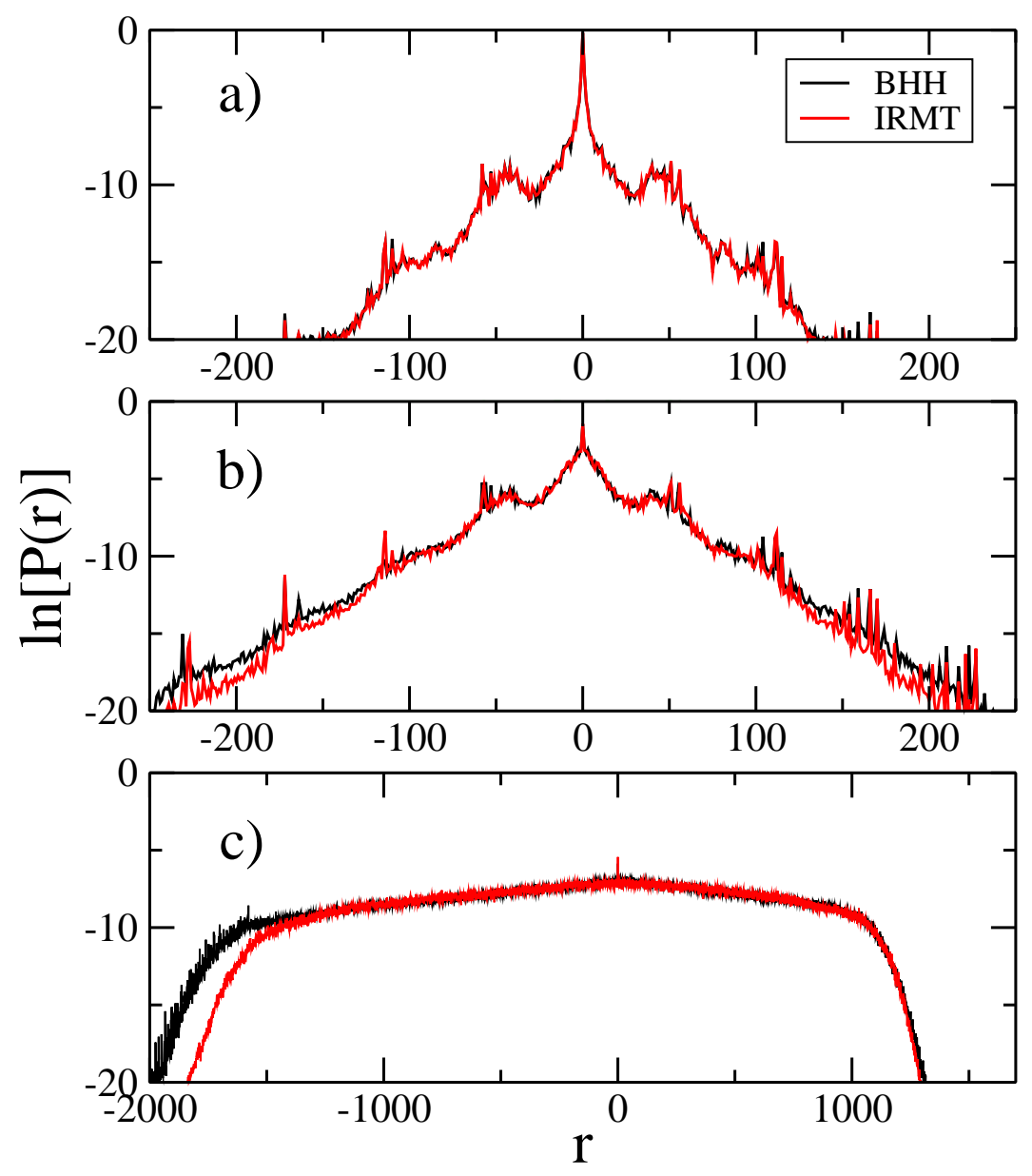

Figure 4.9.: Saturation profiles $P_{\infty}(r)$ of the wavepacket dynamics for the BHH and the IRMT model and various perturbation strengths: (a) $\delta k=0.05$, (b) $\delta k=0.3$, and (c) $\delta k=10$. In the perturbative regimes $(\mathrm{a}, \mathrm{b})$ the agreement with is excellent while in the non-perturbative regime (c) $\delta k>\delta k_{\text {prt }}$ deviations are observed. Here $, N=230, \tilde{U}=280, \tilde{E}=0.26$ and $\lambda_{0}=0.053$.

IRMT modeling. We conclude that the slower decay of $\mathcal{P}(t)$ is due to quantum mechanical effects that still play a prominent role as far as the survival probability is concerned, but otherwise do not significantly affect the profile $P_{t}(r)$.

\subsubsection{The dynamics of the IRMT model}

In this subsection we further investigate the ability of the RMT approach to describe the wavepacket dynamics [57, 137, 112]. At first glance, we might be tempted to speculate that RMT should be able to describe the actual quantum picture at least as far as $\delta E(t)$ is concerned. After all, we have seen in Subsection 4.2.1 that the quantum mechanical LRT formula (4.18) for the energy spreading involves as its only input the classical power spec- 

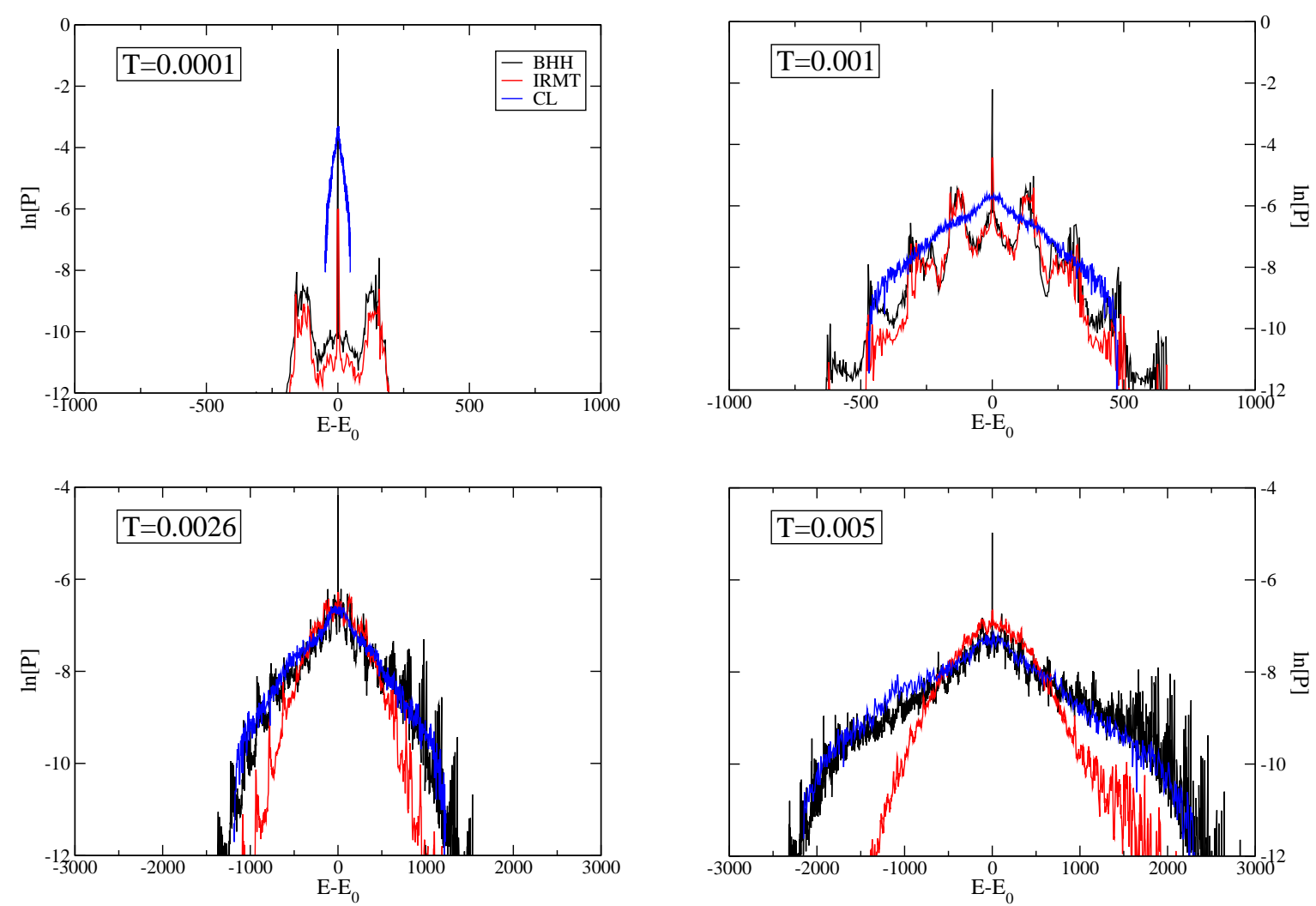

Figure 4.10.: Snapshots of the evolving quantum profile $P_{t}(r)$ obtained from the BHH (black line) and the IRMT model (red line) as well as the classical profile $P_{t}^{\mathrm{cl}}(r)$ in the non-perturbative regime $\delta k=5>\delta k_{\text {prt }}$ plotted against the energy difference $E-E_{0}$. After the quantal transition period $t \sim 0.002$ (see Fig. 4.4b) there is no scale separation between the core and the tail component and one observes overall detailed QCC. However, the initially excited component $\left|n_{0}\right\rangle$ decays slower in the quantum case. Here, $N=230, \tilde{U}=280, \tilde{E}=0.26$ and $\lambda_{0}=0.053$.

trum $\tilde{C}(\omega)$. Thus we would expect that an improved RMT (IRMT) model with the same bandprofile would lead to the same $\delta E(t)$ as in the case of the LDoS studied in Subsection (3.6.2).

However, things are not that simple. In Fig. $4.4 \mathrm{~b}$ we report the numerical results for the spreading $\delta E(t)$ of the IRMT model. ${ }^{9}$ In the standard and in the extended perturbative regimes we observe an excellent agreement with Eq. (4.18). But as soon as we enter the non-perturbative regime, the spreading $\delta E(t)$ shows a qualitatively different behavior than the dynamical BHH model (which follows the prediction of LRT). Namely, after an initial ballistic spreading, we observe a premature crossover to a diffusive behavior

$$
\delta E(t)=\sqrt{2 D_{E} t} .
$$

On the one hand this is not surprising: As we have seen before, the non-perturbative regime

\footnotetext{
${ }^{9}$ The same qualitative results were found also for the prototype WBRM model, see Ref. [57].
} 
corresponds to the semiclassical limit. Therefore, the energy spreading $\delta E(t)$ of the $\mathrm{BHH}$ follows the classical LRT expression (4.14), which is equal to the quantum FOPT expression. The IRMT model does not have a semiclassical limit and accordingly should fail. On the other hand the deviations in $\delta E(t)$ are not expected: We observed in the LDoS studies that the IRMT model was describing the $\delta E$ behavior correctly even in the non-perturbative regime. What is the reason for the deviation in the wavepacket dynamics? Unlike in the LDoS study there is no analytical formula like Eq. (3.33) that would guarantee restricted QCC for any model that shares the same bandprofile as the BHH. In contrast, we are limited to quantum perturbation theory. Once the quantum perturbation theory breaks down also RMT fails to correctly describe the time evolution of the dynamical BHH model.

Let us try to further understand the origin of the diffusive behavior. Up to time $t_{\mathrm{prt}}$ the spreading $\delta E(t)$ is described accurately by the FOPT result (4.18). At $t \sim t_{\text {prt }}$ the evolving distribution becomes as wide as the bandwidth ${ }^{10}$, and we have $\delta E_{\text {core }} \sim \delta E \sim \Delta_{b}$ rather than $\delta E_{\text {core }} \ll \delta E \ll \Delta_{b}$. What happens next? The following simple heuristic picture turns out to be correct. Namely, once the mechanism for ballistic-like spreading disappears, a stochastic-like behavior takes its place. The stochastic energy spreading is similar to a random-walk process where the step size is of the order $\Delta_{b}$, with transient time $t_{\text {prt }}$. In the snapshots shown in Fig. 4.10 the IRMT profile is superimposed as a red line. As one would expect for a random walk, the profile becomes parabolic in the time-regime, where the energy spreading $\delta E(t)$ exhibits diffusion. Therefore we have a diffusive behavior $\delta E(t)^{2}=2 D_{\mathrm{E}} t$ with a diffusion constant $D_{\mathrm{E}}$. For the WBRM model with its sharply defined bandwidth we get

$$
D_{\mathrm{E}}=C \cdot \Delta_{b}^{2} / t_{\mathrm{prt}}=C \cdot \Delta^{2} b^{5 / 2} \delta k \sigma / \hbar \propto \hbar
$$

where $C$ is some numerical pre-factor. This diffusion is not of classical nature, since in the $\hbar \rightarrow 0$ limit we get $D_{E} \rightarrow 0$. The diffusion can persist until the energy spreading profile ergodically covers the whole energy shell and saturates to a classical-like steady state distribution. Accordingly, the differences in the saturation profiles shown in Fig. 4.9c are less pronounced as in the intermediate cases. The time $t_{\text {erg }}$ for which we get ergodization is characterized by the condition $\left(D_{\mathrm{E}} t\right)^{1 / 2}<\delta E_{\mathrm{cl}}$, leading to

$$
t_{\mathrm{erg}}=b^{-3 / 2} \hbar \delta k \sigma / \Delta^{2} \propto 1 / \hbar .
$$

\subsection{Quantum self-trapping in the Bose-Hubbard Hamiltonian}

Complementary to the analysis of the wavepacket dynamics in energy space, we will use this section to shed some light on the time evolution of the $\mathrm{BHH}$ in configuration space.

\footnotetext{
${ }^{10} \mathrm{We}$ recall that in the non-perturbative regime FOPT is subjected to a breakdown before reaching saturation.
} 

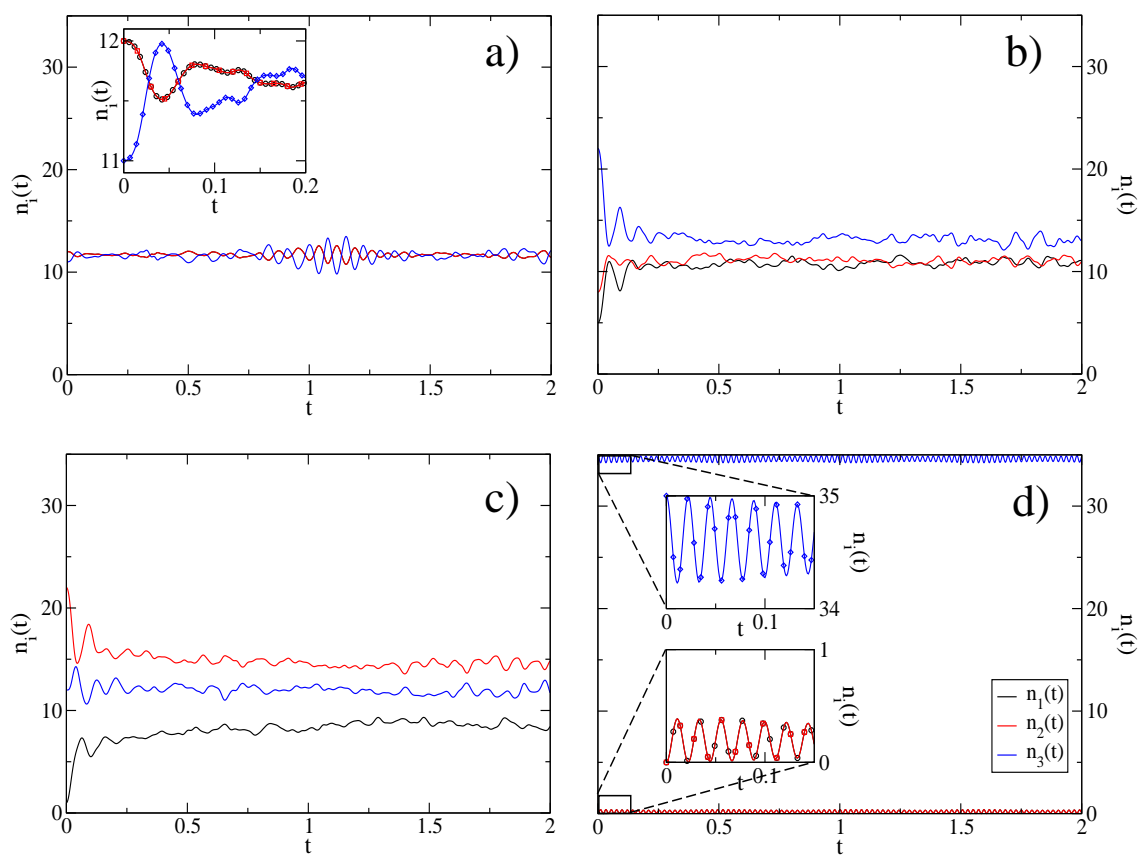

Figure 4.11.: The quantum evolution of $n_{i}(t)$ for various states under the Hamiltonian $\hat{H}_{0}$ starting from the uncoupled Hamiltonian $\hat{H}_{\text {uncpl }}$. The insets show magnifications; there the occupation number $n_{i}(t)$ is additionally marked with symbols: $n_{1}(\circ), n_{2}(\square)$, and $n_{3}(\diamond)$. The number of particles is $N=35, \tilde{U}=280, \lambda_{0}=0$ and $k=15$. The energy of the initial state $\left|n_{0}\right\rangle$ is (a) $\tilde{E}=0.004$ (ground state), (b) $\tilde{E}=0.23$, (c) $\tilde{E}=0.26$, and (d) $\tilde{E}=0.51$ (most excited state). Note that in (a),(d) sites one and two have equal occupation (12 particles)/(zero particles) throughout the entire evolution.

The measure of our interest is the evolving occupation number

$$
n_{i}(t)=\left\langle n_{0}(t)\left|b_{i}^{\dagger} b_{i}\right| n_{0}(t)\right\rangle
$$

where $\left|n_{0}(t)\right\rangle=\hat{U}(t)\left|n_{0}\right\rangle$ is the time-evolved initial state $\left|n_{0}\right\rangle$ (Schrödinger picture). Although these results are not of immediate relevance to the study of the wavepacket dynamics in energy space, they are illuminating as far as the richness of the quantum and classical motion of the $\mathrm{BHH}$ is concerned. The analysis is carried out starting from the basis of the uncoupled Hamiltonian $\hat{H}_{\text {uncpl }}$ with $k_{\text {uncpl }}=k_{0}=0$ where the number of particles in each site is well-defined. The Hamiltonian generating the quantum dynamics is $\hat{H}_{0}=\hat{H}_{\text {uncpl }}-\delta k_{0} \hat{B}$. In order to make a connection with the above studied Hamiltonian $\hat{H}_{0}$ we choose the perturbation strength to be $\delta k_{0}=15$.

We will use initial conditions $\left|n_{0}\right\rangle$ that are determined by different configurations of bosons. For repulsive interaction $U$ - which we consider here - the ground state corresponds to an equidistribution of particles which is due to the nonlinear term in the Bose-Hubbard Hamiltonian 2.10. Accordingly, an unbalanced site-population leads to higher energies and the most excited state corresponds to localization of all particles on one site.

In Fig. 4.11 we present the time evolution of the site occupation numbers $n_{i}(t)$ for four 

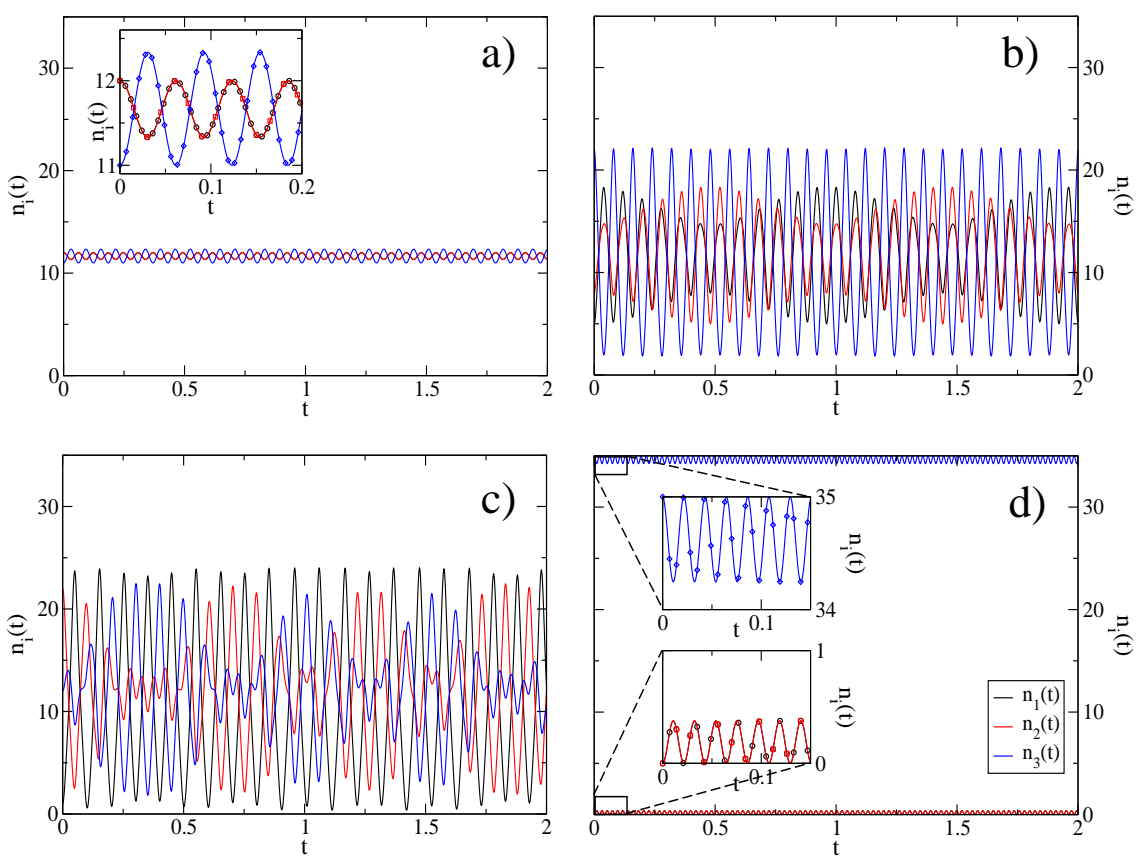

Figure 4.12.: The classical evolution of $n_{i}(t)$ for various initial conditions corresponding to the quantum calculations of Fig. 4.11. The insets show magnifications; there the occupation number $n_{i}(t)$ is additionally marked with symbols: $n_{1}(\circ), n_{2}(\square)$, and $n_{3}(\diamond)$. Note that in (a),(d) sites one and two have equal occupation throughout the entire evolution. The classical dynamics follows the quantum for short times except for the self-trapped regime (d).

representative initial states $\left|n_{0}\right\rangle$ using a system of $N=35$ bosons. Panel (a) shows the ground state of the system. Accordingly, the sites are initially occupied by 12 (sites number one and two) and 11 particles respectively. During the evolution the occupation numbers barely change. As we go higher in the energy $E_{n_{0}}$ of the initial state $\left|n_{0}\right\rangle$ (b,c), one finds the expected increasing imbalance in the site occupation. This imbalance (partly) relaxes during the time evolution leading to an increase in the particle number fluctuations compared to the lower energy (a). However, for very high energies (d) we observe a completely different behavior: The initially extremely unbalanced population (all particles are initially localized on site 3 ) does not relax considerably during the evolution but exhibits only small oscillations.

This phenomenon is known in the literature as "self-trapping" and is a consequence of the nonlinear lattice dynamics. It appears both classically $[23,76]$ and quantum mechanically [215, 151, 89, 63, 155, 1]. In Fig. 4.12 we report the classical evolution of the population $n_{i}(t)$ which corresponds to the non-normalized action $I_{i}(t)$. For short times, quantum and classical calculations agree quite well while for longer times the different nature of quantum and classical dynamics becomes visible. Remarkably, for the self-trapped state also the long-time behavior of classical and quantum dynamics appears to be similar. For a detailed comparison see Refs. [215, 151, 155, 129] and also [4] for some special solutions of the 


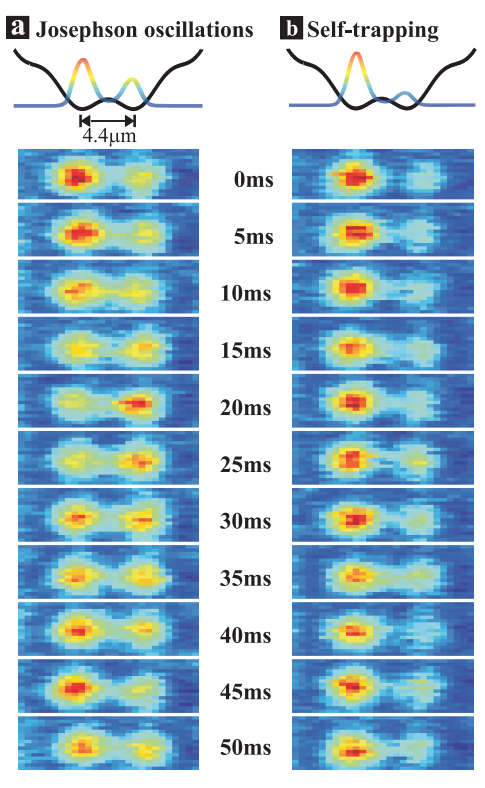

Figure 4.13.: Experimental observation of the tunneling dynamics of two weekly linked Bose-Einstein condensates in a symmetric double-well as indicated by the schematics. The time evolution of the atom population is directly visible in the absorption images. In (a) the initial population difference is below the critical value and hence one observes oscillations of almost all particles. In (b) the initial difference in the population is above the critical value leading to self-trapping of the particles. The figure is a courtesy of M. Oberthaler [1].

classical trimer. Apart from the cited theoretical studies, quantum self-trapping has been recently observed experimentally for the Bose-Hubbard dimer by the group of Oberthaler [1]. In Fig. 4.13 we report their experimental data. Self-trapping can play a crucial role as we shall see in the next chapter on quantum stability [31].

\subsection{Conclusions}

In this chapter we have studied the energy redistribution of the trimeric $\mathrm{BHH}$ model in the chaotic regime as the coupling strength $k$ between neighboring lattice sites undergoes a change $\delta k$ [116]. The specific scenario that we have analyzed in detail is associated with the so-called wavepacket dynamics. While the variance $\delta E^{2}(t)$ of the evolving distribution shows a remarkable quantum-classical correspondence for all $\delta k$-values, higher moments exhibit this QCC only in the non-perturbative $\delta k$-regime identified with the semiclassical limit. Surprisingly, we have found that quantum interference phenomena still dominate the decay of the survival probability $\mathcal{P}(t)$ leading to a slower decay with respect to the classical one. Thus, for $\delta k>\delta k_{\text {prt }}$ detailed QCC $[52,137]$ can be observed with the exception of the central compoent. Using the IRMT model we found that random matrix theory yields a valid description of the quantum dynamics in the perturbative regimes $[137,112]$ but fails in the limit of strong perturbations $\delta k>\delta k_{\text {prt }}$. Finally, we compared the classical and quantum evolution of the on-site population in various dynamical regimes and exhibited the phenomenon of self-trapping $[76,1,92]$. Apart from the interest in understanding the fundamental concept of QCC in the dynamics of quantum chaotic systems, our results are also extremely relevant to bond excitations in small molecules [188] and BECs in optical traps with just a few wells. At the same time, they shed some light on the issue of quantum 
dissipation in BEC due to persistent driving. As to the experimental realization of the latter case, microtrap technology [176] is probably the most promising approach for realizing these small systems. 


\section{Fidelity}

One of the central questions of quantum mechanics is the manipulation of coherence and the stability of complex quantum dynamics under external (environmental) perturbations. This problem has attracted the attention of both the theoretical and the experimental community due to advances in mesoscopic electronic, atomic and molecular physics $[195,118,12]$. The interest is motivated not only by fundamental questions (e.g. quantum-classical correspondence) but also by the possibility to use this knowledge in recent technologies associated with quantum information and quantum computation [157].

The essential ingredient in the discussion of coherence is to identify a system and a bath which interact with one another in such a way that a meaningful distinction between both can be made. In this respect, bosons in optical lattices (OL) - as described by the BoseHubbard Hamiltonian - constitute an ideal framework to study decoherence. On the one hand, these systems are nowadays readily created using standard experimental techniques and realizations of quantum information registers using single/multiple atoms on each site already exist [98]. On the other hand - and maybe even more importantly - these systems allow for an extraordinary degree of control over the system and the bath, as well as over the coupling between them [47]. After having gained insight to the eigenfunctions and the dynamics of the BHH in the previous chapters we are now going to address the issue of coherence and stability of quantum motion within the framework of fidelity.

The fidelity was first introduced by A. Peres [163]. It is defined as

$$
F(t)=\left|\left\langle\psi_{0}\left|e^{+i \hat{H}_{2} t / \hbar} e^{-i \hat{H}_{1} t / \hbar}\right| \psi_{0}\right\rangle\right|^{2},
$$

where $\left|\psi_{0}\right\rangle$ is the initially prepared state and $\hat{H}_{2}=\hat{H}_{1}+\delta k \hat{B}$. The interpretation of (5.1) is twofold: It can be seen as the overlap of an initial state $\left|\psi_{0}\right\rangle$ being once propagated up to time $t$ under the system Hamiltonian $\hat{H}_{1}$, with the same initial state $\left|\psi_{0}\right\rangle$ being propagated for the same amount of time under the Hamiltonian $\hat{H}_{2}$. Here, the term $\delta k \hat{B}$ represents the perturbation due to the interaction with the bath. Under this construction, Eq. (5.1) can be regarded as a way to capture the physical effect of coupling the system to an environment, and hence relate $F(t)$ to decoherence [118]. In this framework, $F(t)$ is also referred to as the Feynman-Vernon influence functional [195]. Alternatively, one can interprete the fidelity as the return probability of a state $\left|\psi_{0}\right\rangle$ which is let to evolve under $\hat{H}_{1}$ up to time $t$ and is then evolved backwards in time under the (slightly) perturbed Hamiltonian $\hat{H}_{2}$ until $t=0$. This viewpoint is commonly adopted in quantum chaos studies where $F(t)$ is a measure for the reversibility of quantum motion [163, 126, 124, 101].

This chapter is organized as follows: First, we are going to discuss the fidelity in terms of decoherence. As a pedagogical example we will borrow the Aharonov-Bohm interference 


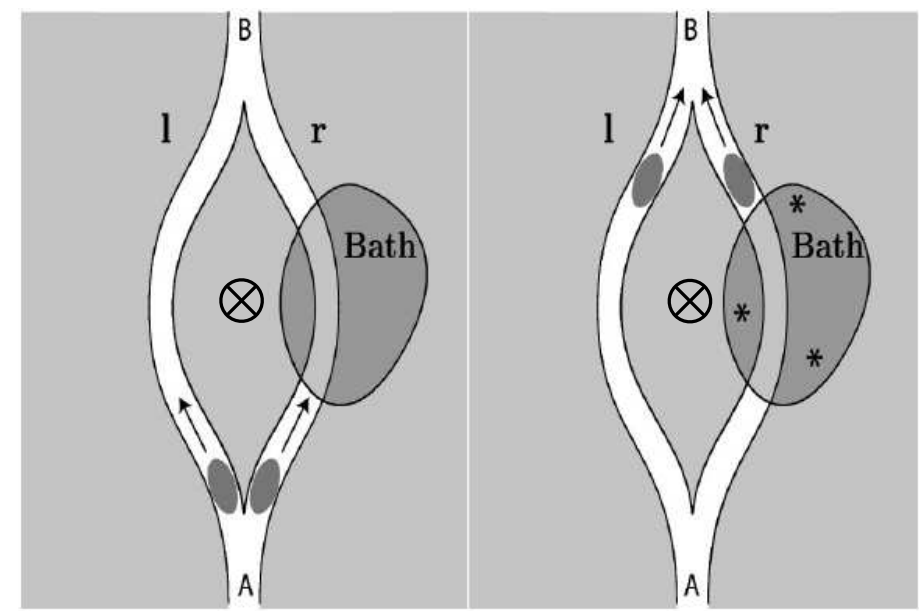

Figure 5.1.: Schematical description of an interference experiment in an Aharonov-Bohm ring: a particle travels through a ring geometry with a perpendicular magnetic field from point $A$ to point $B$ where the interference is measured. It is assumed that the interaction of this system with the bath occurs only along the right path. If no coupling with the bath is present, the interference remains unaffected. If on the other hand the system is coupled to the bath, the interference pattern is changed due to the interaction. Figure taken from [86].

experiment from electronic physics. We then revise how the fidelity $F(t)$ is connected to the stability and the reversibility of quantum dynamics as it was introduced by Peres. In Section 5.3 we derive the theoretical predictions for the fidelity decay in our model, the Bose-Hubbard Hamiltonian. In the last part we present our numerical findings [31]: The most striking feature is the appearance of fidelity echoes at multiples of the time $t_{\text {echo }}$. We identify the underlying lattice dynamics to be the origin of the echo mechanism and demonstrate how to control the echo efficiency by an appropriate choice of the initial preparation. The chapter ends with a conclusion.

\subsection{Fidelity and decoherence}

In theory one can study quantum systems that are perfectly isolated from the environment. Experimentally this is extremely difficult, if not impossible. The question then arises how the environment influences the outcome of the experiment. In order to gain some insight to this fundamental question we are going to make a short detour and "borrow" a well-known paradigm from electronic physics, the Aharonov-Bohm ring, which is especially suited to illuminate the concept of decoherence. The underlying principle is then readily applied to the bosons in OLs, i.e. the BHH.

The prototype example of an interference experiment in the Aharonov-Bohm (AB) ring $[195,86]$ is schematically depicted in Fig. 5.1: a charged particle travels through a ring geometry from point $A$ to point $B$ taking either the left or the right way. Inside the ring is 
a perpendicular magnetic field and it is further assumed that the interaction of this system with the bath occurs only along the right path and that the back-reaction of the bath on the system is small [195]. Since this is a two-path experiment we expect to observe an interference pattern at the point $B .{ }^{1}$ At time $t=0$ the experiment can be described by a wavefunction which is the direct product of the system's state (superposition of a particle taking the left path $l(x, t)$ and the right path $r(x, t))$ and the initial state of the bath $\chi_{0}(\eta)$, where $\eta$ are its internal degrees of freedom:

$$
\psi_{A}(t=0)=[l(x=A, t=0)+r(x=A, t=0)] \otimes \chi_{0}(\eta) .
$$

At the point $x=B$ the wavefunction is given by

$$
\psi_{B}\left(t_{0}\right)=l\left(B, t_{0}\right) \otimes \chi_{l}(\eta)+r\left(B, t_{0}\right) \otimes \chi_{r}(\eta)
$$

which takes into account that the bath's state will evolve differently depending on the path of the particle. The interference term is given by:

$$
2 \mathfrak{R}\left[l^{*}\left(B, t_{0}\right) r\left(B, t_{0}\right)\right] \int \mathrm{d} \eta \chi_{l}^{*}(\eta) \chi_{r}(\eta)
$$

where the integration is done over the bath's degrees of freedom. ${ }^{2}$ If no coupling with the bath is present then $\chi_{l}=\chi_{r}$, hence the second term in 5.4 is equal to unity and one recovers the expression $2 \Re\left[l^{*}\left(B, t_{0}\right) r\left(B, t_{0}\right)\right]$ of an $\mathrm{AB}$-ring without environment. When the coupling to the bath is turned on, the interference term is multiplied by a factor which takes values between unity and zero (in the latter case the interference pattern is completely lost). In the following discussion we will see that this multiplicative factor is the fidelity amplitude, i.e., its absolute value square is the fidelity as defined in Eq. (5.1). The interpretation of this factor is twofold: One can take either the perspective of the system (in this case the electron) or the perspective of the bath. In the former case one sees that the partial wave $r(x, t)$ - when traveling through the bath - experiences a potential. Therefore $r(x, t)$ acquires an additional phase $\phi$ which depends on the possible dynamics and the state of the bath. In the extreme case, this phase is $\pi / 2$ and the interference is lost. Taking the perspective of the bath, one argues that if the bath is not affected by the moving particle, then the interference pattern remains unchanged while in the opposite case the state of the bath is affected in such a way that one can determine the path the particle took. In turn, the uncertainty in the path - which is the origin of the interference - is lost and so is the interference pattern. In the following we are going to discuss both the case of a static bath as well as the case of a bath with internal dynamics following the ideas of Stern, Aharonov, and Imry [195].

\footnotetext{
${ }^{1}$ This can be seen as an analogy of the double slit experiment where instead of the position on the screen now the strength of the magnetic field is used to explore the interference pattern.

${ }^{2}$ This integration is done because only the system can be measured directly while one has no knowledge about the state of the bath, hence one sums over all its possible states and thus "traces" over the bath.
} 


\subsubsection{Static bath}

In the first example, we will consider that the bath consists only of a spin-1/2 particle, i.e., a scatterer which has no dynamics by itself. This spin is located on the right arm of the ring and the interaction takes place within a range $l$ leading to an interaction time $\tau=l /\left(p_{e} / m_{e}\right)$ where $p_{e}$ and $m_{e}$ are momentum and mass of the electron respectively. The spin-electron interaction is modeled by an Ising-like coupling $V_{0} \hat{\sigma}_{z}$. If the spin at time $t=0$ is in an eigenstate of $\hat{\sigma}_{z}$ then the scattering is said to be elastic and the interference term is multiplied by a phase vector. If on the other hand the spin is initially in the eigenstate, say, $\sigma_{x}=+1$ then the scattering process will change the state of the spin and the process is said to be inelastic. In the following we shall see how this leads to dephasing.

At time $t=0$ the total wave function is

$$
\psi(t=0)=\frac{1}{\sqrt{2}}\left[[l(x=A, t=0)+r(x=A, t=0)] \otimes\left[\left|\sigma_{z}=+1\right\rangle+\left|\sigma_{z}=-1\right\rangle\right]\right] .
$$

After passing the scatterer the wave function evolves as

$$
\begin{aligned}
\psi(t)=\frac{1}{\sqrt{2}}\left[l ( x , t ) \otimes \left[\left|\sigma_{z}=+1\right\rangle\right.\right. & \left.+\left|\sigma_{z}=-1\right\rangle\right] \\
& \left.+r(x, t) \otimes\left[e^{-i V_{0} \tau}\left|\sigma_{z}=+1\right\rangle+e^{+i V_{0} \tau}\left|\sigma_{z}=-1\right\rangle\right]\right],
\end{aligned}
$$

and at the point $B$ the interference term is

$$
\cos \left(V_{o} \tau\right) 2 \Re\left[l^{*}\left(B, t_{0}\right) r\left(B, t_{0}\right)\right],
$$

hence it is reduced by the factor $\cos \left(V_{0} \tau\right)$. Let's look at the most drastic case $V_{0} \tau=\pi / 2$. Taking the perspective of the bath, the effect of the interaction was to change the state of the scatterer from $\left|\sigma_{x}=+1\right\rangle$ to $\left|\sigma_{x}=-1\right\rangle$ thus the wave function of the system is

$$
\psi\left(t_{0}\right)=l\left(B, t_{0}\right) \otimes\left|\sigma_{x}=+1\right\rangle+r\left(B, t_{0}\right) \otimes\left|\sigma_{x}=-1\right\rangle,
$$

and the interference is completely lost. If we take the electron's point of view, then we look only at the wave function of the partial waves

$$
l\left(B, t_{0}\right)+e^{i \phi} r\left(B, t_{0}\right) .
$$

In this picture, the right partial wave has accumulated a statistical phase which is distributed as

$$
P(\phi)=\left\{\begin{array}{l}
0.5 \text { for the phase to be }-V_{0} \tau \\
0.5 \text { for the phase to be }+V_{0} \tau
\end{array} .\right.
$$

In oder to calculate the interference term one has to perform an averaging over the possible phases. This leads to four terms and eventually back to Eq. (5.7). Due to the periodicity of the exponential function, $V_{0} \tau=\pi / 2$ is the broadest distribution of phases which can occur and which leads to a total loss of coherence. 


\subsubsection{Dynamical bath}

In the second example, we will consider a bath with (possibly complex) dynamics generated by the Hamiltonian $\hat{H}_{\text {bath }}(\eta)$ and an interaction given by $\hat{V}\left(x_{r}(t), \eta\right)$. Here $x_{r}(t)$ denotes the position of the electron and indicates that the interaction takes place only on the right half of the ring. Starting with the initial wavefunction (5.2) we obtain the total wavefunction at the point $B$

$$
\begin{aligned}
\psi_{B}\left(t_{0}\right)=l\left(B, t_{0}\right) \otimes e^{-i \hat{H}_{\mathrm{bath}}(\eta) t_{0}} \chi_{0}(\eta) & \\
& +r\left(B, t_{0}\right) \otimes \hat{T} \exp \left[-i \int_{0}^{t_{0}} \mathrm{~d} t\left(\hat{H}_{\mathrm{bath}}(\eta)+\hat{V}\left(x_{r}(t), \eta\right)\right)\right] \chi_{0}(\eta),
\end{aligned}
$$

where $\hat{T}$ is the time-ordering operator. By using the interaction picture (with respect to the unperturbed bath evolution $\left.\hat{H}_{\text {bath }}(\eta)\right)$ we obtain for th potential $\hat{V}_{I}\left(\left(x_{r}(t), \eta\right), t\right)=$ $e^{i \hat{H}_{\text {bath }} t_{0}} \hat{V}\left(x_{r}(t), \eta\right) e^{-i \hat{H}_{\text {bath }} t_{0}}$ and with this

$$
\begin{aligned}
\psi_{B}\left(t_{0}\right)=l\left(B, t_{0}\right) \otimes & e^{-i \hat{H}_{\text {bath }}(\eta) t_{0}} \chi_{0}(\eta) \\
& +r\left(B, t_{0}\right) \otimes e^{-i \hat{H}_{\text {bath }}(\eta) t_{0}} \hat{T} \exp \left[-i \int_{0}^{t_{0}} \mathrm{~d} t \hat{V}_{I}\left(\left(x_{r}(t), \eta\right), t\right)\right] \chi_{0}(\eta) .
\end{aligned}
$$

As before, the interference term is multiplied by a factor and takes the form

$$
\left\langle\chi_{0}\left|e^{+i \hat{H}_{\text {bath }}(\eta) t_{0}} e^{-i \hat{H}_{\text {bath }}(\eta) t_{0}} \hat{T} \exp \left[-i \int_{0}^{t_{0}} \mathrm{~d} t \hat{V}_{I}\left(\left(x_{r}(t), \eta\right), t\right)\right]\right| \chi_{0}\right\rangle,
$$

in which one immediately recognizes the fidelity amplitude. Taking the perspective of the bath, the fidelity can be seen in the following way: If the particle takes the left path, the bath evolves under its own dynamics generated only by the Hamiltonian $\hat{H}_{\text {bath }}$. Accordingly we obtain $\left|\chi_{l}\right\rangle=e^{-i \hat{H}_{\text {bath }}(\eta) t_{0}}\left|\chi_{0}\right\rangle$. If on the other hand the particle takes the right path, the evolution of the bath is changed by a perturbation $\hat{V}_{I}$ leading to $\left|\chi_{r}\right\rangle=$ $e^{-i \hat{H}_{\text {bath }}(\eta) t_{0}} \hat{T} \exp \left[-i \int_{0}^{t_{0}} \mathrm{~d} t \hat{V}_{I}\left(\left(x_{r}(t), \eta,\right) t\right)\right]\left|\chi_{0}\right\rangle$ and hence the overlap of the two states is reduced. From the electron's point of view we would again look only at the partial wave functions (5.9). However, now the accumulated phase has a more complex distribution since it depends not only on the initial state of the bath $\chi_{0}(\eta)$ and the time $\tau$ of the interaction but also on the internal dynamics generated by $\hat{H}_{\text {bath }}$. The effect of the interaction of the electron with the bath is then to multiply the interference term with the expectation value of the phase $\left\langle e^{i \phi}\right\rangle=\left\langle\chi_{0}\left|\hat{T} \exp \left[-i \int_{0}^{t_{0}} \mathrm{~d} t \hat{V}_{I}\left(\left(x_{r}(t), \eta,\right) t\right)\right]\right| \chi_{0}\right\rangle$. In order to calculate this average one has also to trace over the bath, since its initial state is unknown. Then, for broad and slowly varying distributions $P(\phi)$ this average is likely to be zero, thereby causing decoherence. 


\subsection{Fidelity and quantum irreversibility}

As mentioned above, the fidelity $F(t)$ was first introduced by Peres [163] who used it to study quantum-classical correspondence. In particular, he showed that the stability - and hence the reversibility - of the quantum dynamics strongly depends on whether the underlying classical motion is chaotic or integrable. Together with the fidelity, he introduced a new concept of irreversibility which is applicable to both quantum and classical dynamics $^{3}$. The intuitive meaning of irreversibility is that there are dynamical evolutions which can easily be prepared, but it is extremely difficult (we say impossible) to prepare the timereversed evolutions. For example, let us consider the melting process of an ice cube floating in a cup of hot water. Let us assume that after some time, we reverse the velocities of all the molecules. If the external conditions are kept strictly the same, the ice cube is expected to re-emerge out of the water. In practice, the initial conditions (fields) are not exactly the same, and as a result we have what looks like irreversibility.

The classical explanation given to irreversibility is based on the concepts of mixing and coarse graining. Mixing can be understood formally in the following way: Consider two finite (and fixed) subsets of phase space, say $V_{1}$ and $V_{2}$, whose measure are fractions $\mu_{1}$ and $\mu_{2}$ of the total phase space. Suppose that at the time $t_{1}$, the distribution $f_{1}(p, q)$ is uniform in $V_{1}$ with $\int \mathrm{d} V_{1} f_{1}=1$. Then, for any time $t_{2}$ sufficiently remote from $t_{1}$ (in the future or in the past) and for sufficiently large $\mu_{1}$ and $\mu_{2}$ we have $\left|\int \mathrm{d} V_{2} f_{2}-\mu_{2}\right|<\delta$, with arbitrarily small $\delta$, irrespective of where $V_{1}$ is. Obviously, the smaller $\mu_{1}$ or $\mu_{2}$, the larger the time $\left|t_{1}-t_{2}\right|$ needed for mixing. In the ice-cube paradigm, $V_{1}$ (the region of phase space which corresponds to an ice cube in hot water) is considerably smaller than $V_{2}$, which corresponds to what we loosely call lukewarm water with no further specification of the water properties. This is because,for a given total energy, nearly all the points of phase space are compatible with this vaguely specified state of water, so that $\mu_{2} \approx 1$. Therefore, almost every dynamical evolution will lead to lukewarm water, with extremely small inhomogeneities, since $\mu_{2} \approx 1$ is the probability of finding the final state in $V_{2}$. Nevertheless we can, conceptually, prepare lukewarm water at time $t_{2}$ so that, at a later time $t_{1}$ it will separate into an ice cube and hot water. But this requires a very special preparation: Not only a cup of lukewarm water, but one with precise correlations between all the molecules. This preparation is restricted to lie in an exceedingly contorted region of phase space. With such small structures, mixing will not yet have accomplished after given finite time $t_{1}-t_{2}$.

The property of mixing is time-symmetric and by itself it cannot explain irreversibility. Now comes coarse graining. We are unable, with our imperfect instruments, to prepare the initial state in such a way that it will reside within a region of phase space that is so small or that has such a complicated structure that mixing will not yet have occurred after a finite time $t_{1}-t_{2}$. That is, we cannot prepare the system at time $t_{2}$ so that after a finite time $t_{1}-t_{2}$ it will be located with certainty within the desired domain $V_{1}$ of phase space. There are evolutions (as from lukewarm water to an ice cube in hot water) that cannot be made to

${ }^{3}$ Here we follow closely the discussion of Peres in Ref. [164] 


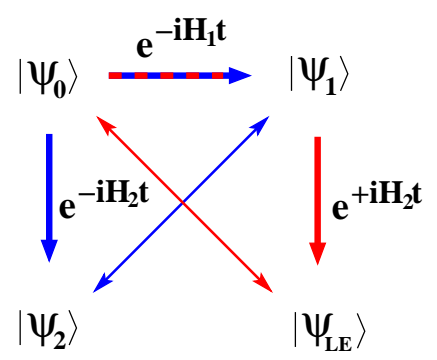

Figure 5.2.: Schematic representation of the two (mathematically equivalent) notions of $F(t)$ : the absolute value squared of the bracket between $\left|\psi_{1}\right\rangle$ and $\left|\psi_{2}\right\rangle$ yields the fidelity while the from bracketing $\left|\psi_{0}\right\rangle$ and $\left|\psi_{\mathrm{LE}}\right\rangle$ one obtains the Loschmidt echo.

proceed. What happens to mixing and coarse graining if we go to the quantum world?

First of all, a distribution in phase space cannot develop structures on scales smaller than $\hbar$ due to the uncertainty principle. Therefore it does not lead to the mixing property as we defined it above. Moreover, coarse graining of instruments does not seem to make sense in the quantum context. Here, dynamical quantities appear with discrete values and it is, in principle, possible to prepare arbitrary, pure quantum states. To make things worse, if there is an uncertainty in the preparation of the initial state it will not grow, because the time evolution is unitary. This implies that initially neighboring states remain close throughout the evolution, as their scalar product is invariant.

In order to introduce a concept for analyzing the stability of both classical and quantum motion, Peres made the following ansatz: Instead of assuming an initial preparation $\left|\psi_{0}\right\rangle$ of limited accuracy, he assumed that the Hamiltonian is not known exactly because there is no way to have complete control over the system's parameters, for example a stray magnetic or gravitational field present in the lab. This led to the above definition of the fidelity $F(t)$ (see Eq. 5.1) which can be interpreted in the following two different, though formally equivalent, ways (see Fig. 5.2): In order to determine the stability of the quantum dynamics one has to calculate the overlap of an initial state $\left|\psi_{0}\right\rangle$ being once propagated under the system Hamiltonian $\hat{H}_{1}$, with the same initial state $\left|\psi_{0}\right\rangle$ being propagated under the perturbed Hamiltonian $\hat{H}_{2}$. This is Peres' original interpretation of fidelity and is denoted by the 'blue route' in the above scheme. It has been recently used, for example, in quantum computation studies [157] to quantify the corruption of quantum information. Alternatively, one can interprete the fidelity in the following way ('red route'): The state $\left|\psi_{0}\right\rangle$ prepared at $t=0$ is let to evolve for a time $t>0$ under the Hamiltonian $\hat{H}_{1}$. The resulting state is then evolved backwards under the Hamiltonian $\hat{H}_{2}$ till the time $t=0$. Since the system is not isolated from the environment (bath) there are unavoidable differences between the Hamiltonians $\hat{H}_{1}$ and $\hat{H}_{2}$ and therefore $F(t)$ is expected to decay as the time $t$ increases. In this interpretation $F(t)$ is the return probability, a measure for the reversibility of the dynamical evolution. This is the picture described by Ref. [126] which was inspired by nuclear magnetic resonance experiments [161]. These experiments explore the scenario that -under certain circumstances- it is possible to evolve a complex quantum system backwards in time. This follows the spirit of the gedanken experiment at the origin of the Boltzmann-Loschmidt controversy [16] and for that reason $F(t)$ is also called Loschmidt echo. 


\subsection{Fidelity of cold atoms in an optical lattice: Theoretical background}

In this section we present the theoretical predictions for the temporal decay of the fidelity $F(t)$ of cold atoms loaded in an optical lattice or coupled micro-traps subject to perturbations of the coupling: $k_{0} \rightarrow k_{0} \pm \delta k$ [31]. In the context of OLs this perturbation is readily achieved by adjusting the intensity of the laser beams that create the lattice. In our analysis, we consider that

$$
\hat{H}_{1,2}=\hat{H}_{0} \mp \delta k \hat{B} .
$$

As in Chapters 3 and 4, the unperturbed Hamiltonian $\hat{H}_{0}$ is given by Eq. (2.39) with $k=k_{0}$ and $\hat{B}$ is the coupling operator (see Eq. 3.2). ${ }^{4}$ This perturbation is similar to a momentum boost which has recently been investigated [165] in the context of the fidelity. It was found that - as long as the boost is not too large - the fidelity freezes at some finite value. In the numerical analysis we will use $k_{0} \approx 15$ and $\tilde{U} \approx 280$. If not stated otherwise the number of particles is $N=230$. It is a fixed assumption throughout this work that the perturbation is classically small $\delta k \ll \delta k_{\mathrm{cl}}$, i.e., the corresponding classical Hamiltonians ${ }^{5} \mathcal{H}_{0}, \mathcal{H}_{1}$, and $\mathcal{H}_{2}$ are generators of classical dynamics of the same nature. Thus we are always in the classical LRT regime.

The main tool of the analytical considerations will be quantum linear response theory. To this end we rewrite the fidelity from Eq. (5.1) as

$$
F(t)=\left|\left\langle\psi_{2}^{S}(t) \mid \psi_{1}^{S}(t)\right\rangle\right|^{2}
$$

where the superscript " $S$ " indicates the Schrödinger picture $i \hbar \partial_{t}\left|\psi_{i}^{S}(t)\right\rangle=\hat{H}_{i}\left|\psi_{i}^{S}(t)\right\rangle$ with $i=1,2$. It is useful to change to the interaction picture $\left|\psi_{i}^{S}(t)\right\rangle=e^{-i \hat{H}_{0} t / \hbar}\left|\psi_{i}(t)\right\rangle$. The initially prepared states at $t=0$ are $\left|\psi_{i}^{S}(0)\right\rangle=\left|\psi_{i}(0)\right\rangle=|\psi(0)\rangle$. Expanding the Born-series up to second order we obtain

$$
\begin{aligned}
& \left|\psi_{1}(t)\right\rangle=|\psi(0)\rangle+i \frac{\delta k}{\hbar} \int_{0}^{t} \mathrm{~d} t^{\prime} \hat{B}\left(t^{\prime}\right)|\psi(0)\rangle-\left(\frac{\delta k}{\hbar}\right)^{2} \int_{0}^{t} \mathrm{~d} t^{\prime} \int_{0}^{t_{1}} \mathrm{~d} t^{\prime \prime} \hat{B}\left(t^{\prime}\right) \hat{B}\left(t^{\prime \prime}\right)|\psi(0)\rangle \\
& \left|\psi_{2}(t)\right\rangle=|\psi(0)\rangle-i \frac{\delta k}{\hbar} \int_{0}^{t} \mathrm{~d} t^{\prime} \hat{B}\left(t^{\prime}\right)|\psi(0)\rangle-\left(\frac{\delta k}{\hbar}\right)^{2} \int_{0}^{t} \mathrm{~d} t^{\prime} \int_{0}^{t_{1}} \mathrm{~d} t^{\prime \prime} \hat{B}\left(t^{\prime}\right) \hat{B}\left(t^{\prime \prime}\right)|\psi(0)\rangle
\end{aligned}
$$

With the substitutions

$$
\hat{I}(t)=\int_{0}^{t} \mathrm{~d} t^{\prime} \hat{B}\left(t^{\prime}\right) ; \quad \hat{J}(t)=\int_{0}^{t} \mathrm{~d} t^{\prime} \int_{0}^{t^{\prime}} \mathrm{d} t^{\prime \prime} \hat{B}\left(t^{\prime}\right) \hat{B}\left(t^{\prime \prime}\right)
$$

\footnotetext{
${ }^{4}$ Again, we stress the fact that for an appropriate choice of energy and lattice parameters the BHH trimer can be chaotic and thus contains the main ingredient to generalize the result to larger lattices (see Section 2.6). ${ }^{5}$ These are obtained from Eq. 2.23 with $v_{i}=0$ and $f=3$.
} 
and using the notation $\langle\cdot\rangle_{0}=\langle\psi(0)|\cdot| \psi(0)\rangle$ we obtain for the fidelity:

$$
\begin{aligned}
& F(t)=\left|\left\langle\psi_{2}^{S}(t) \mid \psi_{1}^{S}(t)\right\rangle\right|^{2} \\
& =\left|\left\langle\psi_{2}(t)|\underbrace{e^{i H_{0} t / \hbar} e^{-i H_{0} t / \hbar}}_{=1}| \psi_{1}(t)\right\rangle\right|^{2} \\
& =\mid\left(\langle\psi(0)|+i \frac{\delta k}{\hbar}\langle\psi(0)| \hat{I}(t)-\left(\frac{\delta k}{\hbar}\right)^{2}\langle\psi(0)| \hat{J}(t)\right) \\
& \times\left.\left(|\psi(0)\rangle+i \frac{\delta k}{\hbar} \hat{I}(t)|\psi(0)\rangle-\left(\frac{\delta k}{\hbar}\right)^{2} \hat{J}(t)|\psi(0)\rangle\right)\right|^{2} \\
& =\mid 1+2 i \frac{\delta k}{\hbar}\langle\hat{I}(t)\rangle_{0}-2\left(\frac{\delta k}{\hbar}\right)^{2}\langle\hat{J}(t)\rangle_{0}-\left(\frac{\delta k}{\hbar}\right)^{2}\left\langle\hat{I}^{2}(t)\right\rangle_{0}-i\left(\frac{\delta k}{\hbar}\right)^{3}\langle\hat{I}(t) \hat{J}(t)\rangle_{0} \\
& -i\left(\frac{\delta k}{\hbar}\right)^{3}\langle\hat{J}(t) \hat{I}(t)\rangle_{0}+\left.\left(\frac{\delta k}{\hbar}\right)^{4}\left\langle\hat{J}^{2}(t)\right\rangle_{0}\right|^{2} \\
& =1-4\left(\frac{\delta k}{\hbar}\right)^{2}\langle\hat{J}(t)\rangle_{0}-2\left(\frac{\delta k}{\hbar}\right)^{2}\left\langle\hat{I}^{2}(t)\right\rangle_{0}+4\left(\frac{\delta k}{\hbar}\right)^{2}\langle\hat{I}(t)\rangle_{0}^{2}+O\left(\left(\frac{\delta k}{\hbar}\right)^{3}\right)(5.1
\end{aligned}
$$

Expanding the wave functions $\psi_{i}$ to higher order and summing up the terms leads to the exponentiation of the linear response result

$$
F(t)=1-\left(2 \frac{\delta k}{\hbar}\right)^{2} J(t)+\cdots \approx e^{-\left(2 \frac{\delta k}{\hbar}\right)^{2} J(t)},
$$

where $J(t)=\langle\hat{J}(t)\rangle_{0}$. Eq. (5.18) can be interpreted in terms of a fluctuation-dissipation relationship [172]. On the left hand side we have the fidelity which describes dissipation of quantum information and on the right hand side we have an integrated time-correlation function, i.e., a fluctuating quantity. Another immediate observation by direct inspection of (5.18) is that the stronger the correlations decay the slower will the fidelity decay and vice versa $[173,101]$. This is somehow counterintuitive as it states that the more chaotic a system is, the slower is the decay of the fidelity. For classically regular systems on the other hand, the correlator will generally not decay to zero and thus $F(t)$ will decay much faster.

From Eq. (5.18) it is clear, that the only ingredient in the perturbation theory is the correlator $C(\tau)$. As we have seen in Chapter 3, the information of $C(\tau)$ is fully encoded in the bandprofile $\tilde{C}(\omega)$ of the perturbation matrix B (see Eq. 3.14). Thus we expect that the improved random matrix theory model (see Section 3.2) which incorporates the structures in the energy landscape of the perturbation operator $\hat{B}$ [59] but doesn't contain higher order correlations is able to reproduce the LRT results. In Fig. 5.3 we present the results of these simulations. An excellent agreement of the IRMT data with the fidelity calculations of the $\mathrm{BHH}$ (2.39) in the perturbative regime $\delta k<\delta k_{\text {prt }}$ is evident. This is in accord with 


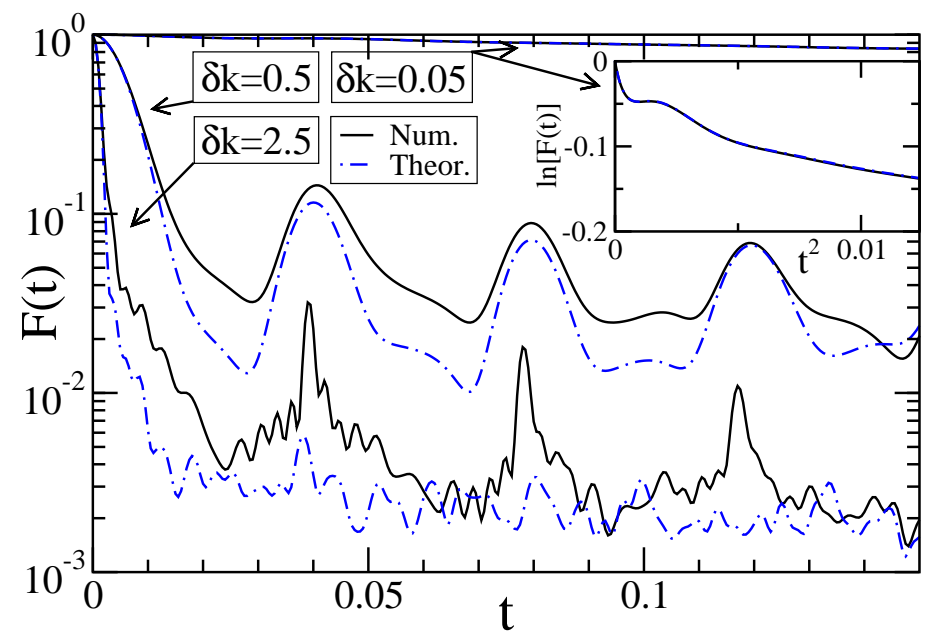

Figure 5.3.: The fidelity $F(t)$ at $\tilde{E}_{n}^{(0)}=0.26$ for three perturbation strengths: $\delta k=0.05<\delta k_{\mathrm{qm}}$ (upper curves and inset), $\delta k=0.5<\delta k_{\text {prt }}$ (middle curves), and $\delta k=2.5>\delta k_{\text {prt }}$ (lower curves). Black solid lines correspond to the exact numerical result obtained from eigenstates of the Hamiltonian $\hat{H}_{0}$ while dash-dotted lines correspond to the theoretical expectation. We have found that within the perturbative regime, the IRMT calculations coincide with the linear response theory expression from Eq. (5.18).

our findings for the IRMT in the LDoS (see Figs. 3.14a, b) and the wavepacket dynamics studies (see Figs. 4.4, 4.7a) presented in the previous two chapters. Therefore we come to the conclusion that within the perturbative regime LRT and IRMT are equivalent not only for "static" quantities (like the LDoS) but also for complex time-evolution schemes (like the fidelity). At the same time the IRMT modeling cannot describe the quantum results in the non-perturbative regime. Here, the echoes are related to subtler correlations of dynamical nature (self-trapping). These go beyond the autocorrelation function $C(\tau)$ that determines the bandprofile and therfore cannot be captured by LRT or the IRMT modeling.

Coming back to Eq. (5.18), we mark that it is not restricted to short times. The condition for its applicability is that the perturbation $\delta k$ is sufficiently small so that $1-F(t) \ll 1$. Specifically, for quantum mechanically small perturbations $\delta k \leq \delta k_{\mathrm{qm}} \sim \Delta / \sigma$ Eq. (5.18) implies a short time Gaussian decay $F(t)=\exp \left[-8 C(0)(\delta k \cdot t / \hbar)^{2}\right]$, which evolves into a long time Gaussian decay (see inset of Fig. 5.3) [101, 99, 124, 20, 66, 172]

$$
F(t) \approx e^{-\left(\frac{\sigma \delta k}{\hbar}\right)^{2} t^{2}} .
$$

The decay of $F(t)$ for small perturbations $\delta k \leq \delta k_{\mathrm{qm}}$ is the same irrespective of the nature (integrable or chaotic) of the underlying classical dynamics. In order to derive the above equation we used the fact that the transitions occur only between neighboring levels. ${ }^{6}$

In the case where the perturbation mixes levels in a distance larger than the mean level

${ }^{6}$ For the evaluation of $C(\tau \approx 0)=\int_{-\omega_{0}}^{\omega_{0}} \mathrm{~d} \omega \tilde{C}(\omega)$ in the standard perturbative regime we assume that $\omega_{0} \sim \frac{\Delta}{\hbar}$. 
spacing $\Delta$ but still smaller than the bandwidth $\Delta_{b}$ (Wigner perturbative regime) $\delta k_{\mathrm{qm}} \leq \delta k \leq$ $\delta k_{\text {prt }} \sim \tilde{U} / N$ we have to distinguish short- and long-time behavior as the correlation function decays sufficiently fast for $t>\tau_{c l}$ while it is assumed to be constant for short times. If the bandprofile of the perturbation operator $\mathbf{B}$ were flat, we would expect $[101,124,20,66$, $172,99]$ an exponential decay $F(t) \sim e^{-2 \Gamma t}$. The rate $\Gamma \sim(\sigma \delta k)^{2} / \Delta$ is given by the width of the Local Density of States (LDoS) [115] studied in Chapter 3. As a result we get

$$
F(t)=\left\{\begin{array}{cc}
1-\left(\frac{\sigma \delta k}{\hbar}\right)^{2} t^{2} \quad \text { for short times } t<\tau_{c l} \\
1-\frac{(\sigma \delta k)^{2}}{\hbar} \frac{t}{\Delta} \approx \exp \left[-\frac{(\sigma \delta k)^{2}}{\hbar} \frac{t}{\Delta}\right] \quad \text { for large times } t>\tau_{c l}
\end{array} .\right.
$$

We note that the parabolic decay is present for any perturbation strength at sufficiently short times.

For even stronger perturbations $\delta k>\delta k_{\text {prt }}$, we enter the semiclassical regime $[124,20$, 66]. As we have seen in Chapter 3 the non-perturbative regime coincides with the semiclassical limit since $\delta k_{\text {prt }} \sim \tilde{U} / N \rightarrow 0$ in the limit $N \gg 1$ (while $\tilde{U}=$ const. [115]). Then for any fixed perturbation $\delta k$, eventually $\delta k \gg \delta k_{\text {prt }}$. In this regime (and provided that the classical dynamics is chaotic) it was found recently $[126,66,214,124,20]$ that the exponential decay persists but that the decay rate approaches some semiclassical value $\gamma_{\mathrm{SC}}$

$$
F(t) \sim e^{-\gamma_{\mathrm{SC}} t} .
$$

In some cases ${ }^{7}, \gamma_{\mathrm{SC}}$ was found to be equal to the classical Lyapunov exponent $\Lambda$ [126]. This result raised a lot of research activity within the last years, since on the one hand the classical dynamics $(\Lambda)$ manifests itself in quantum evolution, and on the other hand suggests a perturbation independent dephasing rate. However, we would like to point out that the study of the general conditions for having a fingerprint of the classical Lyapunov exponent in quantum fidelity experiments is not yet settled and surprises are possible as we will see in the next section.

In the deep non-perturbative regime the quantum-classical correspondence may become strong enough so that one can rely on purely classical calculations. The classical fidelity is then defined as

$$
F_{\mathrm{cl}}(t)=\int_{\Omega} \mathrm{d} \mathbf{x} \rho_{-\delta k}(\mathbf{x}, t) \rho_{+\delta k}(\mathbf{x}, t)
$$

where $\rho_{ \pm \delta k}$ is the classical density function evolved under $\mathcal{H}\left(k_{0} \pm \delta k\right)$ (see footnote 5) and the integration is performed over the whole phase space ${ }^{8} \Omega$. This means that the classical fidelity $F_{\mathrm{cl}}(t)$ is defined as the fractional overlap of the two phase-space densities that result from the propagation of an initial phase-space volume under $\mathcal{H}\left(k_{0} \pm \delta k\right)$ (see Fig. 5.4).

\footnotetext{
${ }^{7}$ In fact, the perturbation-independent decay of $F(t)$ appears only for some special sets of initial states, like narrow wave packets [66, 124]. This has to be contrasted with the perturbative regime $\delta k<\delta k_{\text {prt }}$, where the $F(t)$-decay is qualitatively the same for any initial preparation.

${ }^{8}$ In the actual calculation the integration is done over an energy shell of width $d E$. We have checked that the numerical procedure is stable with respect to $d E$.
} 


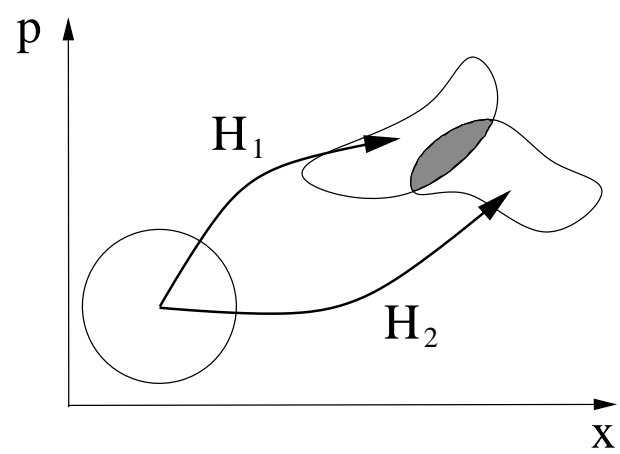

Figure 5.4.: Scheme of the classical fidelity calculation: an initial phase-space volume (circle) is evolved under the two Hamiltonians $H_{1}, H_{2}$. The classical fidelity $F_{\mathrm{cl}}(t)$ is defined as the fractional overlap (grey shaded area) of the two phase-space volumes that result from the propagation.

\subsection{Numerical analysis of the fidelity}

Complementary to the previous section, we here present the numerical studies of the temporal decay of the fidelity $F(t)$ for the Bose-Hubbard Hamiltonian. The perturbation is applied to the coupling between lattice sites: $k_{0} \rightarrow k_{0} \pm \delta k$, hence we consider $\hat{H}_{1,2}=\hat{H}_{0} \mp \delta k \hat{B} .{ }^{9}$ Once again we note that the perturbation is classically small $\delta k \ll \delta k_{\mathrm{cl}}$, thus we are always in the classical LRT regime. Quantum mechanically, we work in the $\hat{H}_{0}$ eigenbasis. The initial preparation $\left|\psi_{0}\right\rangle$ is chosen to be either a single eigenstate of $\hat{H}_{0}$ or a Gaussian superposition of eigenstates centered at energy $E_{0}$. We will investigate the fidelity decay in various energy regimes where the underlying classical dynamics is qualitatively different.

Our main results are summarized in Fig. 5.5 where we plot the fidelity $F(t)$ as function of time for various perturbation strengths $\delta k$. The new striking feature is the appearance of echoes at multiples of $t_{\text {echo }}$. By analyzing the energy landscape of the perturbation operator we are able to identify $t_{\text {echo }}$ and control the echo efficiency by an appropriate choice of the initial preparation. For moderate perturbations $\delta k<\delta k_{\text {prt }}$, linear response theory as discussed in the previous section can reproduce these echoes. This is demonstrated by applying the IMRT modeling which incorporates the semiclassical structures in the energy landscape of the perturbation matrix B. For larger perturbations $\delta k>\delta k_{\text {prt }}$ we rely on the (semi-)classical considerations presented above. We show that the trajectories leading to fidelity echoes become more abundant at high energies, in contrast to recent experimental results on echo spectroscopy on ultra-cold atoms in atom-optics billiards [5] where it was found that the echoes do not survive in the strong perturbation limit. Our analysis indicates that this is due to self-trapping phenomena [132, 215, 174, 75] and reflects a generic property of interacting bosons loaded on a lattice.

\subsubsection{Fidelity decay before the first revival}

We start with the discussion of the fidelity decay before the first revival takes place. For some representative plots of the fidelity see Figs. 5.8(d-f). In the chaotic regime, for small perturbations $\delta k<\delta k_{\text {prt }}$ we observe an initial parabolic (Gaussian) decay which is followed

\footnotetext{
${ }^{9}$ See around Eq. (5.13) for a detailed description of the setup and the used parameters.
} 


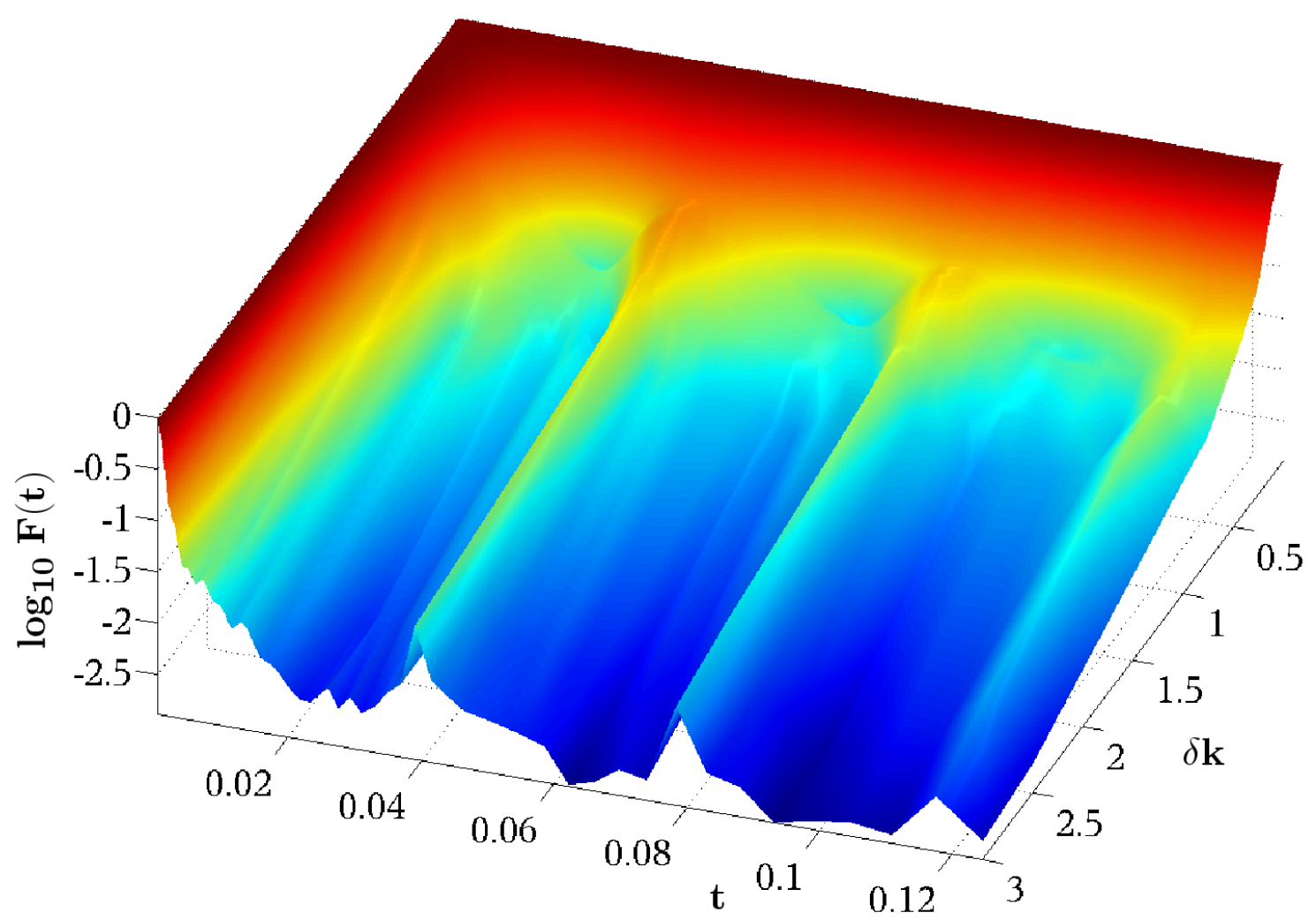

Figure 5.5.: Parametric evolution of the fidelity $F(t)$ for different perturbation strengths $\delta k$ as a function of time. The initial preparation $\left|\psi_{0}\right\rangle$ is an eigenstate of the Hamiltonian $\hat{H}_{0}$ at $\tilde{E}_{n}^{(0)}=0.26$. The fidelity exhibits echoes at multiples of $t=t_{\text {echo }}$. Here the Heisenberg time is $t_{H} \simeq 0.55$.

by an exponential decay thus confirming the validity of the above theoretical expectations (5.20) for the BHH. In order to quantify this statement we fit the initial fidelity decay with a Gaussian $F(t) \sim \exp \left[-\gamma_{\mathrm{G}} t^{2}\right]$ and the following decay with an exponential function $F(t) \sim$ $\exp \left[-\gamma_{\exp } t\right]$. In Fig. 5.6 we report both decay rates as a function of $\delta k$. For the Gaussian decay we find $\gamma_{\mathrm{G}} \propto \delta k^{2}$ which is in excellent agreement with the theoretical prediction (5.20). Also in case of the exponential decay rates $\gamma_{\exp }$ would have expected a quadratic behavior $\gamma_{\exp } \propto \delta k^{2}$ [124]. This expectation is based on the study of the LDoS which was discussed in Chapter 3: In the perturbative regime the LDoS is assumed to have a Lorentzian shape (3.24) with a width $\Gamma \sim \delta k^{2} / \Delta$ reflecting Fermi's golden rule. Although the LDoS shape of the BHH is not strictly Lorentzian ${ }^{10}$ one can still detect the general features (coretail structure). In Chapter 3 the core-width $\Gamma$ of the LDoS for our specific model was found to scale as $\Gamma \propto \delta k^{\alpha}$ with $\alpha=1.9 \pm 0.1$. However, we observe deviations from the quadratic behavior of $\gamma_{\text {exp }}$ especially for larger $\delta k$ values. We attribute them to the fitting process

\footnotetext{
${ }^{10}$ The Lorentzian shape is a special case of the simplified WBRM model which assumes a flat bandprofile (see Section 3.4).
} 


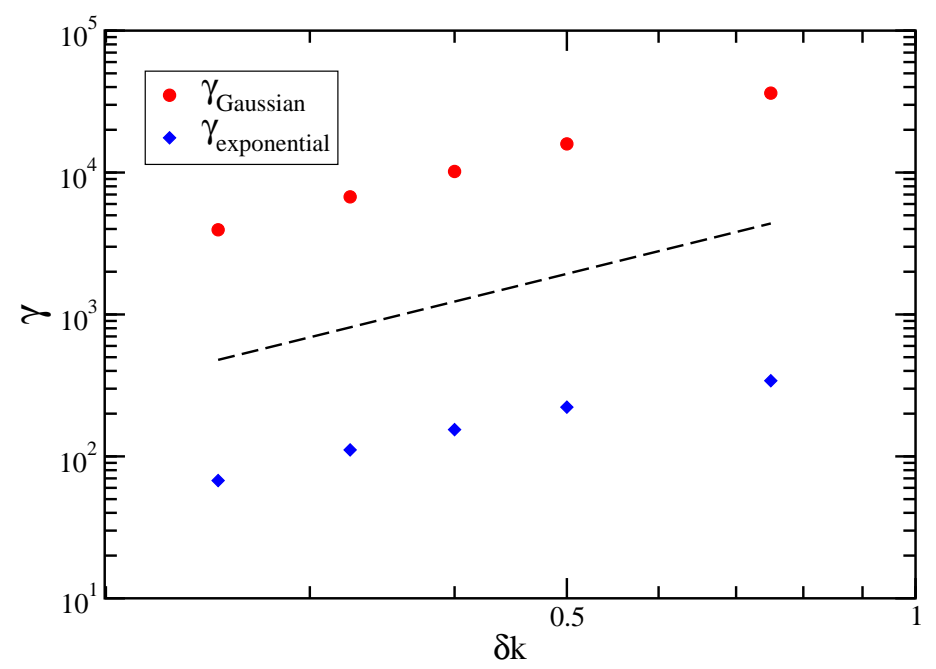

Figure 5.6.: The decay rates $\gamma$ of the fidelity $F(t)$ for an initial eigenstate of $\hat{H}_{0}$ in the Wigner perturbative regime for $\tilde{E}_{n}^{(0)}=0.26$ (chaotic regime). The circles correspond to the initial Gaussian decay while the diamonds represent the decay rates of the exponential decay that follows the initial Gaussian. The dashed line is drawn to guide the eye and has slope 2 .

which is prone to errors in the regime of large perturbations for the system sizes used here.

In the remainder of this subsection we will focus on the non-perturbative regime. We start with the discussion of the chaotic regime. Here, we observe that for $\delta k>\delta k_{\text {prt }}$ the initially fast exponential fidelity decay is followed by a (slower) second exponential decay (see inset of Fig. 5.7). The corresponding decay rates $\gamma_{1,2}$ are shown in Fig. 5.7 (the dashed lines have slope 1 and are drawn to guide the eye). While $\gamma_{1}$ increases linearly with the perturbation strength $\delta k$, the decay rate $\gamma_{2}$ saturates to the Lyapunov decay of the underlying classical dynamics. This behavior results from two different mechanisms: The fast initial decay originates from the deformation of the energy surfaces. The width $\delta E$ of the resulting energy shell is the largest energy scale of the system; it is given by the distance of the unperturbed Hamiltonian $\hat{H}_{0}$ from the Hamiltonians $\hat{H}_{1,2}$ yielding $\delta E \sim \delta k$ and consequently $\gamma_{1} \sim \delta k$ [214]. The second mechanism becomes relevant, once the energy shell is explored. Then, the further evolution depends mainly on the underlying classical dynamics of the system and the fidelity decay rate is given by the maximum Lyapunov exponent [126].

Less clear is the situation for (predominantly) integrable systems for perturbation strengths $\delta k>\delta k_{\text {prt }}$ : Depending on the initial state, the fidelity decays either faster than Gaussian (when the perturbation changes the frequencies of the phase-space tori) $[173,170]$, or in a power law fashion, when the primary effect of the perturbation is to change the shape of the phase-space tori [123]. Indeed, our numerical results for the BHH in the predominantly integrable regime (high energies) confirm the above expectation (see Figs. 5.8f, 5.9). In the latter figure we overplot also the classical fidelity $F_{\mathrm{cl}}(t)$. Although the quantum and classical calculations - as far as the echoes are concerned - agree quite well (see next sub- 


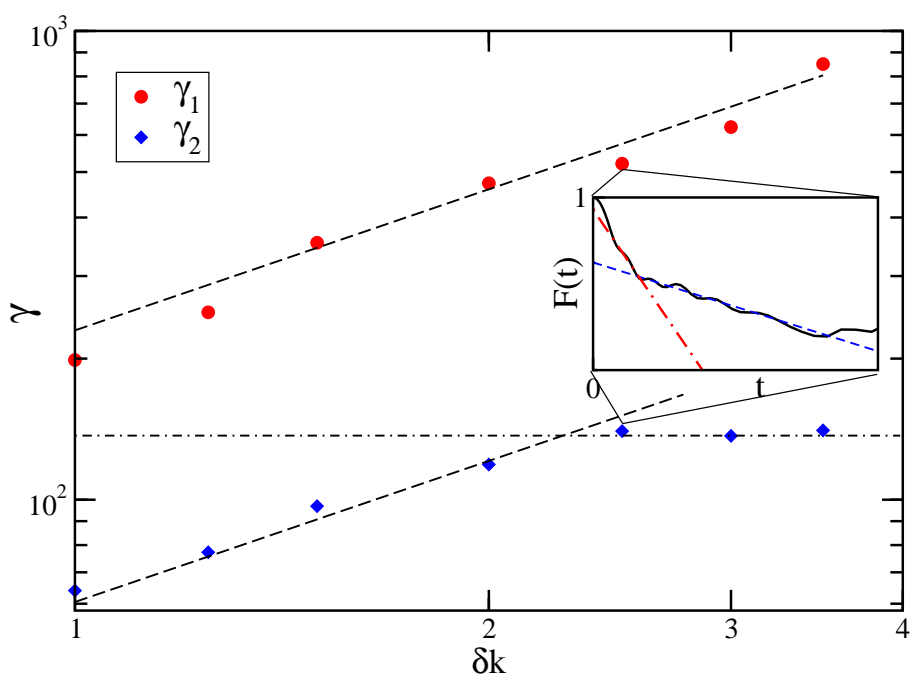

Figure 5.7.: The decay rates $\gamma_{1,2}$ of the fidelity $F(t)$ for the initial eigenstate of $\hat{H}_{0}$ in the nonperturbative regime for $\tilde{E}_{n}^{(0)}=0.26$. The dashed lines have slope 1 and are drawn to guide the eye. The inset shows an exemplary decay of $F(t)$ for $\delta k=2.5$.

section), a surprising discrepancy in the decay between $F(t)$ and $F_{\mathrm{cl}}(t)$ for small times is also evident. In contrast to the chaotic regimes (lower energies), where for $\delta k>\delta k_{\text {prt }}$ the fidelity decay was exponential with a rate given by the classical Lyapunov exponent, here

$$
F_{\mathrm{cl}}(t) \sim t^{-\alpha}
$$

while the quantum fidelity decays as

$$
F(t) \sim t^{-3 \alpha / 2}
$$

The power law decay is a signature of classically (predominantly) integrable dynamics while the anomalous (faster) quantum decay is a pure quantum phenomenon, as was pointed out in [123]. According to the prediction of [124], the classical power law exponent had to be $\alpha=d$, where $d=3$ is the dimensionality of the system. In fact, in the case of the BHH trimer model (2.23) we have a six-dimensional phase space with two constants of motion, namely the total number of particles $N$ and the energy $E$ and thus $d_{\text {eff }}=2 .^{11}$ However, in the high energy regime, the dynamics is dictated by self-trapping phenomena, leading to localization of particles in one site (see also Section 4.4). Therefore the effective dimensionality of the system described by the Hamiltonian (2.23) is $d_{\mathrm{eff}}=1$. Indeed, the best linear fit to the numerical data yields $\alpha=1$, thus confirming the above argument. 


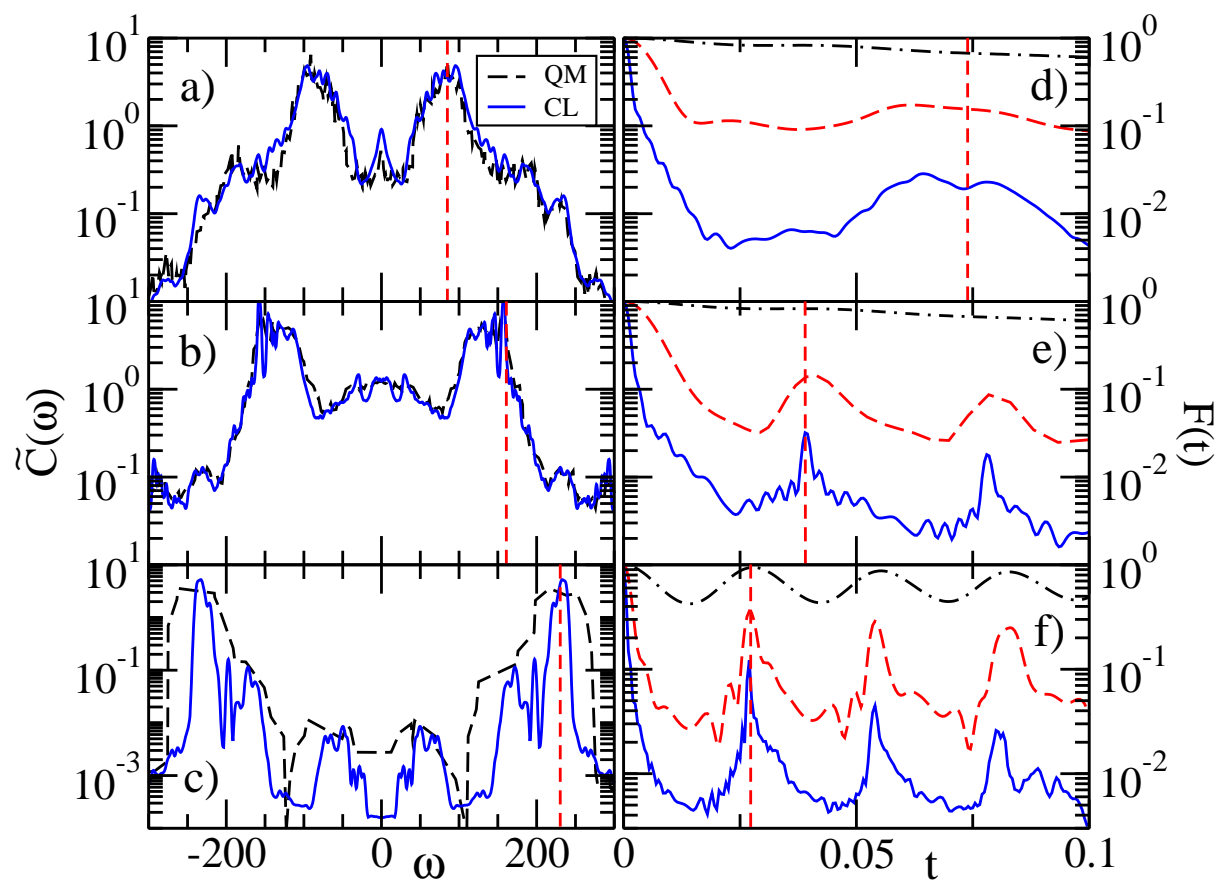

Figure 5.8.: The quantum bandprofile $\left\langle\left|\mathbf{B}_{n m}\right|^{2}\right\rangle \tilde{U} / N^{2} \Delta$ and classical power spectrum $\tilde{C}(\omega)$ (drawn with dashed and solid lines respectively) at various eigenvalues $\tilde{E}_{n}^{(0)}$ of the reference Hamiltonian $H_{0}$ : a) $\tilde{E}_{n}^{(0)}=0.22$, b) $\tilde{E}_{n}^{(0)}=0.26$, c) $\tilde{E}_{n}^{(0)}=0.39$. In panels d)-f) we plot the corresponding fidelity $F(t)$ for three representative values $\delta k<\delta k_{\mathrm{qm}}$ (dashed-dotted line, deep perturbative regime), $\delta k_{\mathrm{qm}}<$ $\delta k<\delta k_{\text {prt }}$ (dashed line, perturbative regime), and $\delta k>\delta k_{\text {prt }}$ (solid line, non-perturbative regime). The respective numerical values are for d) and e) $\delta k=0.1,0.5,2.5$, and for f) $\delta k=0.5,2.5,7.5$. The initial preparation is an eigenstate of $H_{0}$. The vertical dashed lines in sub-figures d)-f) denote the revival time $t_{\text {echo }}$ while in a)-c) they denote the corresponding frequency $\omega_{\text {echo }}=2 \pi / t_{\text {echo }}$.

\subsubsection{Fidelity echoes}

As discussed previously, a striking feature is the appearance of echo revivals in the fidelity $F(t)$. These are not trivial recurrences due to the discreteness of the spectrum [198]. Rather they reflect the underlying classical dynamics of the system. In order to gain some insight we turn first to the analysis of the perturbation matrix $\mathbf{B}$ which generates the dynamics. In Figs. 5.8(a-c) we show the bandprofile $\left\langle\left|\mathbf{B}_{n m}\right|^{2}\right\rangle_{E}$ (dashed line) for various energy regimes $E$. As seen in the last chapters the bandprofile is not flat, but exhibits pronounced structures within the bandwidth $\Delta_{b}$. Furthermore we observe that the position $\omega_{\text {echo }}$ of the side-bands increases as we increase the energy $E$. As a result the correlator $C(\tau)$ (see Eqs. (5.17, 5.18)) oscillates, leading to strong fidelity echoes at multiples of a characteristic time $t_{\text {echo }}=$ $2 \pi / \omega_{\text {echo }}$. In Figs. 5.8(d-f) the revival time $t_{\text {echo }}$ is denoted by vertical dashed lines while in the left column of Fig. 5.8 they represent the corresponding frequency $\omega_{\text {echo }}$. One clearly

\footnotetext{
${ }^{11}$ This is in agreement with the $\hbar$-dependence of the perturbative borders $\delta k_{\mathrm{qm}}$ and $\delta k_{\mathrm{prt}}$ found for the LDoS.
} 


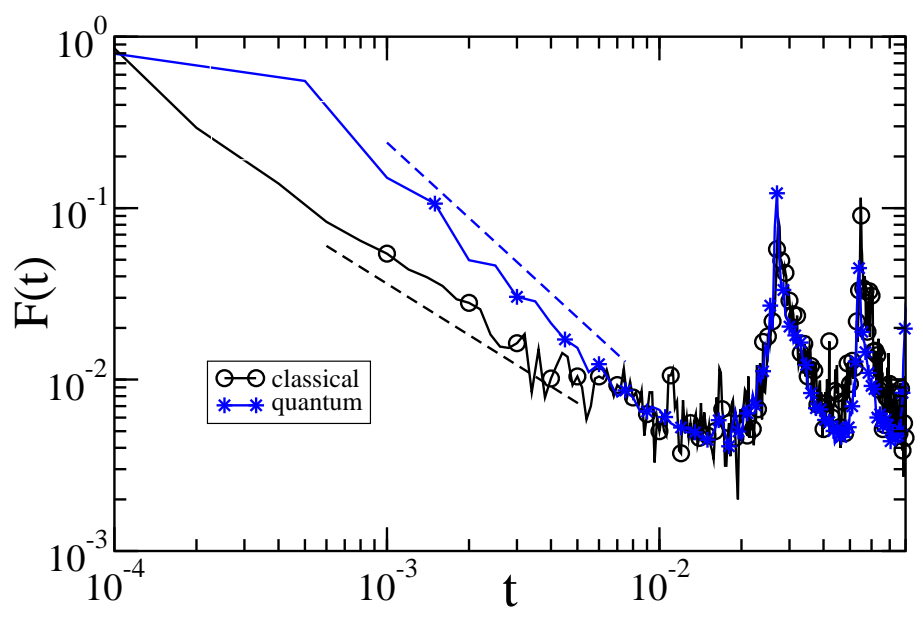

Figure 5.9.: The quantum (blue line) fidelity decay $F(t)$ at $\tilde{E}_{n}^{(0)}=0.39$, plotted together with the corresponding classical (black line) fidelity decay $F_{\mathrm{cl}}(t)$, calculated from Eq. (5.22). The perturbation strength is $\delta k=7.5>\delta k_{\text {prt }}$. The dashed lines are the best least square fits and are drawn to guide the eye. The upper one corresponds to $t^{-1.46}$ while the lower one corresponds to $t^{-0.99}$.

observes the agreement of $\omega_{\text {echo }}$ with the position of the side-bands. These echoes are different from the standard mesoscopic echoes at the Heisenberg time $t_{\mathrm{H}}=1 / \Delta$ of quantum systems with chaotic classical dynamics [198]. They are instead associated with "nonuniversal" structures that dominate the bandprofile of the perturbation matrix $\mathbf{B}$ and are the fingerprints of the lattice confinement. Quantum mechanically, these are reflected in selection rules that determine to which states $\left|n\left(k_{0} \pm \delta k\right)\right\rangle$ of $H_{1,2}$ an initial state $\left|\psi_{0}\right\rangle$ is coupled.

We consider the case where the initial preparation $\left|\psi_{0}\right\rangle=\left|m\left(k_{0}\right)\right\rangle$ is an eigenstate of $H_{0}$ corresponding to an eigenvalue $E_{m}^{(0)}$. Here, the information about the coupling of the initial states to the perturbed states $\left|n\left(k_{0} \pm \delta k\right)\right\rangle$ is encoded in the structure of the LDoS (see Eq. (3.20)). For $\delta k \leq \delta k_{\text {prt }}$ the LDoS has a core-tail structure (3.24) with a core-width $\Gamma \approx(\sigma \delta k)^{2} / \Delta \leq \Delta_{b}$ indicating the energy regime where most of the probability is contained [115]. For short times $t \leq \Gamma^{-1}$, this core will not have time to dephase/decay. Therefore, the fidelity will show large echo recoveries at the time the side-bands have acquired a phase of $2 \pi$, if $t_{\text {echo }} \ll \Gamma^{-1}$. The numerical data for $\delta k \leq \delta k_{\text {prt }}$ reported in Figs. 5.8(d-f) (see dashed lines) confirm this prediction. We see that the echo efficiency (i.e. the recovery level of $F(t)$ ) increases as $t_{\text {echo }}$ becomes smaller. ${ }^{12}$ Arguing along the same lines as above, we are able to explain the reduction of fidelity echoes in the non-perturbative regime, $\delta k>\delta k_{\text {prt }}$, observed in Figs. 5.8(d-f) (see solid lines). Here, the LDoS covers the whole bandwidth, spoiling (with respect to the perturbative cases) the echo efficiency.

Similar echoes were experimentally observed for atoms in optical billiards [5] (see

\footnotetext{
${ }^{12}$ We have checked that in the cases presented in Figs. 5.8(d-f) the width $\Gamma$ remains approximately the same for corresponding $\delta k$-values.
} 

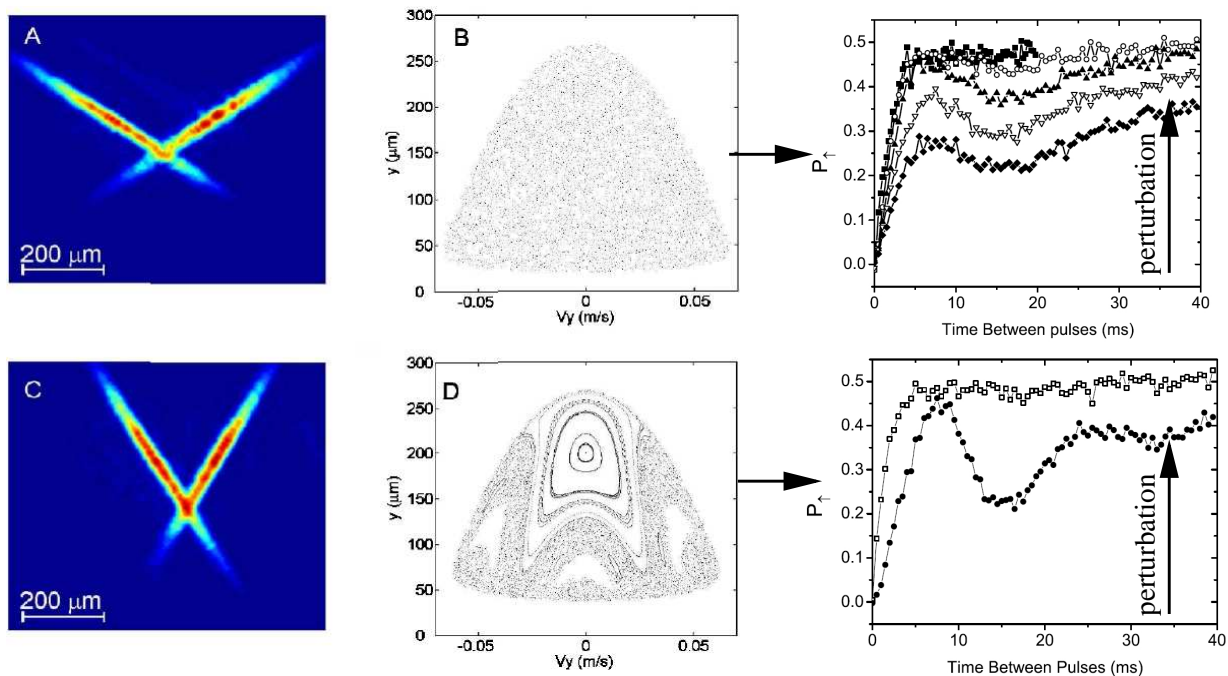

Figure 5.10.: Experimental study of the fidelity using atoms in an optical billiard (taken from [6]). The atoms are trapped in a "light-sheet wedge billiard" (panels A,C) consisting of to intersecting laser beams which represent an effective potential for the atoms (the trap is elongated in the direction perpendicular to the paper-plane, the atoms are loaded from the top). Depending on the intersection angle of the lasers, the underlying classical phase space is chaotic (B) or mixed (D). The timereversal evolution scheme is imposed using microwave spectroscopy. In the right panels the amount of particles in the upper state $P_{\uparrow}(t)$ is plotted for various perturbation strengths. In this setup $1-P_{\uparrow}(t)$ is proportional to the fidelity amplitude [5,6]. Revivals are observed for both chaotic and mixed phase space, but disappear in the limit of strong perturbations.

Fig. 5.10). However, in contrast to our case (see black lines in Figs. 5.8d-f), Ref. [6] was reporting a total loss of echoes in the semiclassical regime $\delta k \geq \delta k_{\text {prt }}$. Looking at Fig. 5.9 we observe that quantum and classical fidelity calculations agree quite well as far as the echoes are concerned (the discrepancy on the fidelity decay between $F(t)$ and $F_{\mathrm{cl}}(t)$ for small times was discussed in the previous subsection). In the following we will use the classical fidelity $F_{\mathrm{cl}}(t)$ to shed some light on the origin of the echoes observed in the $\mathrm{BHH}$ model (2.39) for $\delta k \geq \delta k_{\text {prt }}$.

The classical trajectories contributing to the ensemble average of the echo are those that - after evolving forward in time with $\mathcal{H}_{1}$ and then backwards in time with $\mathcal{H}_{2}$ - return to the vicinity of their initial position. Since $\mathcal{H}_{1}$ and $\mathcal{H}_{2}$ are different in the coupling between nearby wells, those classical trajectories that do not jump between wells will not feel the difference in the coupling terms. Therefore they retrace their forward propagation backwards in time, causing the action integral to vanish. These trapped trajectories give a perfect contribution to the echo signal. Their existence is due to both the discreteness and the nonlinearity of the underlying equations of motion. For high enough energies the configurations where bosons are localized in one lattice site persist for long times as can be seen from Fig. 5.11. In such cases the bosons are said to be "self-trapped". As a result, 

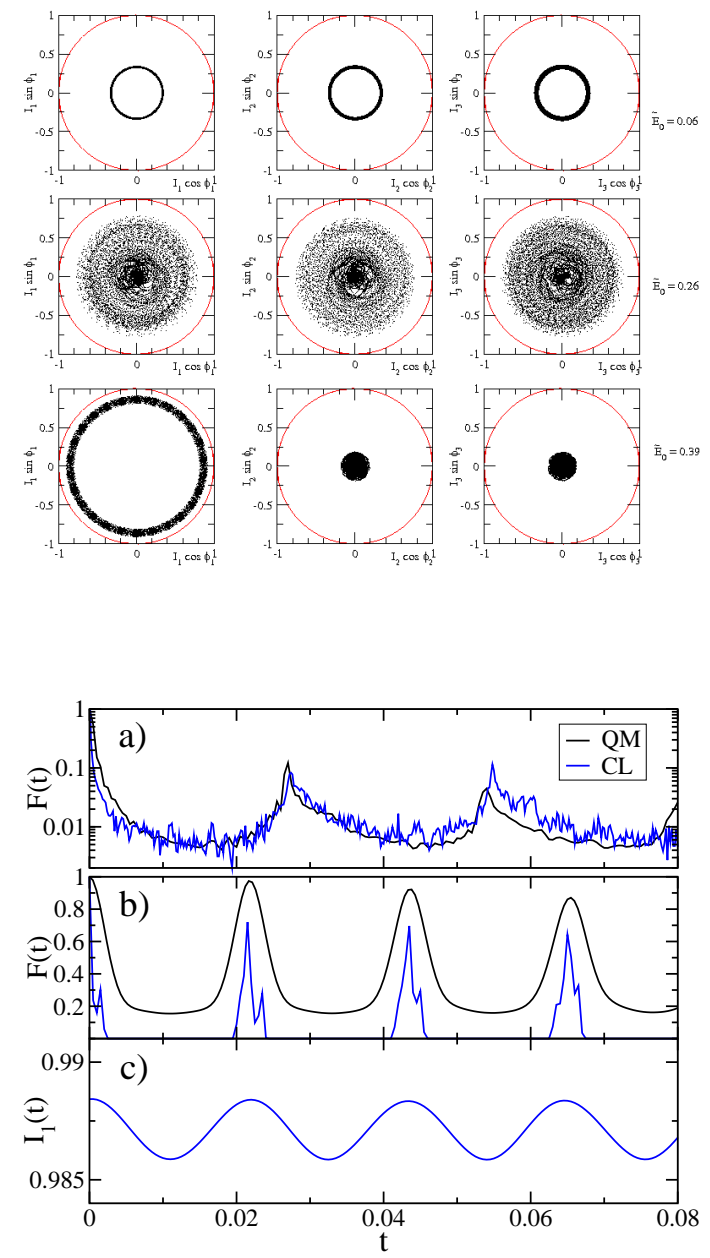

Figure 5.11.: Time-evolution of $N=230$ particles (bosons) associated with various energy configurations: (a) $\tilde{E}=0.06$ close to the ground state (equipartition) of the system; (b) $\tilde{E}=0.26$ corresponding to a state in the middle of the energy spectrum and (c) $\tilde{E}=0.39$ associated with a high energy state. The red line indicates the accessible phase space if all particles were on the corresponding site. Note that as the energy increases, the likelihood of "trapping" particles, i.e. particles that are initially localized in one site (in the specific case $i=1$ ) and stay there for long times, increases.

Figure 5.12.: The quantum (blue line) fidelity decay $F(t)$ plotted together with the corresponding classical (black line) fidelity decay $F_{\mathrm{cl}}(t)$, calculated from Eq. (5.22) for the energies $\tilde{E}_{n}^{(0)}=0.39$ (a), $\tilde{E}_{n}^{(0)}=0.5(\mathrm{~b})$. The perturbation strength is in both cases $\delta k=7.5>\delta k_{\text {prt }}$. In the lower panel (c) we plot the classical action $I_{1}(t)$ (i.e., the normalized population at site \#1) corresponding to the data in panel (b). One observes that the oscillations in the population coincide with the revivals of the fidelity. The different degree of quantumclassical agreement in panels (a),(b) results from a stronger numerical averaging in the upper panel.

the echo efficiency is increased in agreement with the numerical simulations presented in Figs. 5.8(d-f). We note that for even higher energies one can achieve an echo efficiency of almost a 100\%: In Fig. 5.12 we compare the the data from Fig. 5.9 (upper panel) with the results for the most excited states of the system at $\tilde{E}=0.5$ (middle panel). In the lower panel of Fig. 5.12 we report the corresponding normalized population $I_{1}(t)$ of site 1 . One observes that these highly excited states represent system configurations where almost all particles are trapped in one site (here site \#1) throughout the time evolution exhibiting only small fluctuations in the site occupation.

Finally, we are going address the question how the echo efficiency is affected if the initial preparation $\left|\psi_{0}\right\rangle$ is not a single delta state. To this end we calculate the fidelity $F(t)$ for the case where $\left|\psi_{0}\right\rangle$ is a superposition of eigenstates of $\hat{H}_{0}$ with a Gaussian weight around some energy $E_{0}$. The numerical data for both the perturbative and the non-perturbative regime and energy $\tilde{E}_{0}=0.26$ are reported in Fig. 5.13. For comparison, we overplot the previous calculation for a single eigenstate of $\hat{H}_{0}$ with a solid black line. One observes that as the 

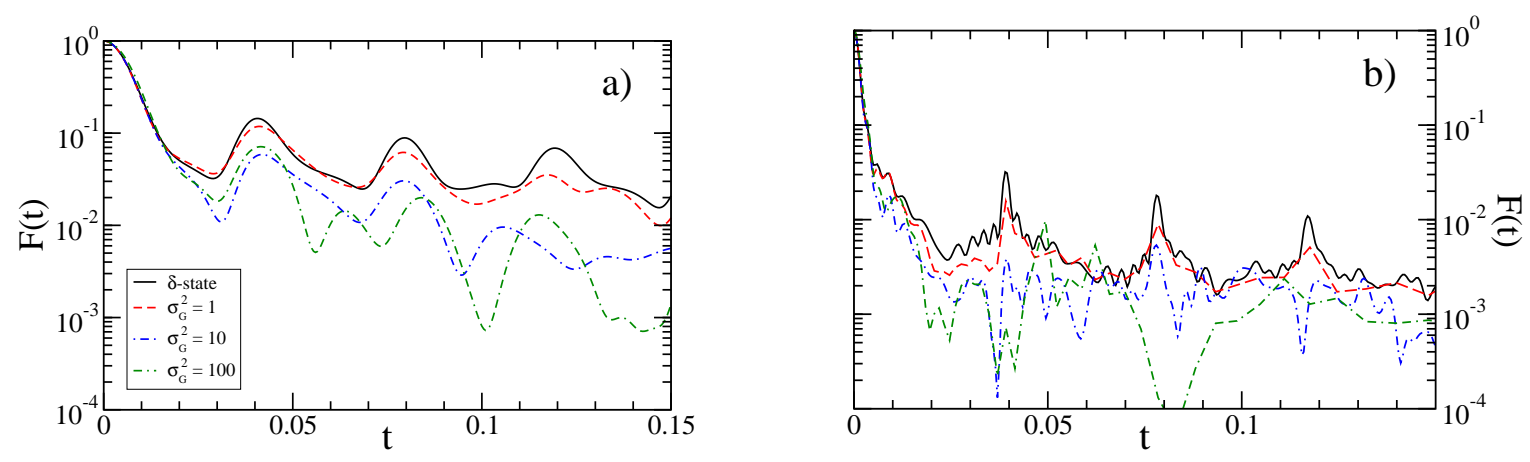

Figure 5.13.: The fidelity $F(t)$ at $\tilde{E}_{n}^{(0)}=0.26$ for various initial conditions and $\delta k=0.5<\delta k_{\text {prt }}$ (a), $\delta k=2.5>\delta k_{\text {prt }}$ (b). The black solid line corresponds to a delta initial state while the dashed lines correspond to Gaussian states centered around the same energy $\tilde{E}_{n}^{(0)}$ with variance $\sigma_{\mathrm{G}}^{2}=1$ (red dashed), $\sigma_{\mathrm{G}}^{2}=10$ (blue dash-dotted), and $\sigma_{\mathrm{G}}^{2}=100$ (green double-dash-dotted) respectively. One observes that the echo-efficiency is suppressed as the variance $\sigma_{\mathrm{G}}^{2}$ is increased. In the nonperturbative regime (b) the effect becomes more pronounced. See text for details.

width $\sigma_{\mathrm{G}}^{2}$ of the Gaussian preparation increases the echo-efficiency is decreased. Once the width $\sigma_{\mathrm{G}}^{2}$ is large enough such that the participating eigenstates of $H_{0}$ span an energy window which is wider than $\delta E$ over which the bandprofile of $\mathbf{B}$ is constant (see for example Figs. 5.8a-c), the echo is suppressed. This is due to destructive interferences resulting from states located at various parts of the spectrum where the band-structure - and accordingly $\omega_{\text {echo }}-$ is different. The decrease in the echo efficiency becomes more pronounced in the non-perturbative regime where already for small variances $\sigma_{\mathrm{G}}^{2}$ no sign of echo is observed (see Fig. 5.13b).

\subsection{Conclusions}

In this chapter we have studied the fidelity decay $F(t)$ for the Bose-Hubbard Hamiltonian. In particular, we have found a new striking feature, namely the appearance of fidelity echoes at multiples of $t_{\text {echo }}$ which persist from the FOPT $\left(\delta k<\delta k_{\mathrm{qm}}\right)$ to the deep nonperturbative regime $\left(\delta k>\delta k_{\text {prt }}\right)$. By analyzing the energy landscape of the perturbation operator $\mathbf{B}$ we were able to identify $t_{\text {echo }}$ and control the echo efficiency by an appropriate choice of the initial preparation. For moderate perturbations $\delta k<\delta k_{\text {prt }}$, the IRMT modeling which incorporates the semiclassical structures of the perturbation operator could reproduce these echoes, while for larger perturbations $\delta k>\delta k_{\text {prt }}$ we relied on the classical fidelity $F_{\mathrm{cl}}(t)$. We showed that the trajectories leading to fidelity echoes become more abundant at high energies, in contrast to recent experimental results on echo spectroscopy on ultra-cold atoms in atom-optics billiards [5] where it was found that the echoes do not survive in the strong perturbation limit. Our analysis indicates that this is due to self-trapping phenomena $[132,215,174,75]$ and reflects a generic property of interacting bosons loaded on a 
lattice. Therefore the reported results are expected to be applicable for larger lattices $f>3$ as well, although the above analysis is focused on the $f=3$ case. Furthermore, due to the connection between fidelity decay and loss of coherence $[65,100]$, our findings can be used to engineer coherence echoes of a central system coupled to a quantum bath consisting of cold atoms in an optical lattice [5, 179]. 


\section{Quantum Pumping}

A major advantage of BEC based devices, as compared to conventional solid-state structures, lies in the extraordinary degree of precision and control that is available, regarding not only the confining potential, but also the strength of the interaction between the particles, their preparation, and the measurement of the atomic cloud. The realization of atom chips [108, 176, 201], "conveyor belts" [109], atom diodes and transistors [150, 189, 196] is considered a major breakthrough with potential applications in the field of quantum information processing [184], atom interferometry [10, 187, 205] and lasers [149, 107, 133, 191].

Here we propose a BEC stirring device $[113,114]$ based on the BHH trimer which can be regarded as the incorporation of a quantum pump in a closed system: It produces an adiabatic DC current in response to a cyclic change of two control parameters of the (optical) lattice confining the atoms. We believe that the proposed stirring device $[175,145]$ can be realized using to-date optical lattice technology [3]. The actual measurement of induced neutral currents poses a challenge to experimentalists. However, various techniques have been proposed for this purpose. For example one can exploit the Doppler effect at the perpendicular direction, which is known as the rotational frequency shift [29].

Quantum pumping/stirring represents a special case of driven systems and hence is closely related to the notion of parametric Hamiltonians introduced in Chapter 3. In contrast to the previous chapters we consider here adiabatically slow driving where dissipation is not an issue. In this case the generated current is determined by the "adiabatic transport" which is sometimes also referred to as "geometric conductance" in the theory of the Berry phase. We treat the problem using the Kubo formalism. Our results indicate that the resulting atom current can be extremely well controlled. We find $[113,114]$ that the nature of the transport process depends crucially on the sign and on the strength of the interatomic interactions We can distinguish four regimes of dynamical behavior. For strong repulsive interaction the particles are transported one-by-one, which we call sequential crossing. For weaker repulsive interaction we observe either gradual crossing or coherent mega crossing. Finally, for strong attractive interaction the particles are glued together and behave like a huge classical ball that rolls from trap to trap.

This chapter is organized as follows: In the next section we introduce the proposed pumping cycle and its implementation using the BHH trimer model. Then we briefly review the Kubo formula approach to quantum pumping [54] which is based on the theory of adiabatic processes [25, 15, 27]. In Section 6.3 we present our results for the BHH trimer. We begin with the "two-orbital approximation" of a single-particle three-site system and then derive $[113,114]$ analytical expressions for the conductance of the BHH trimer. The chapter ends with a conclusion. 
a)

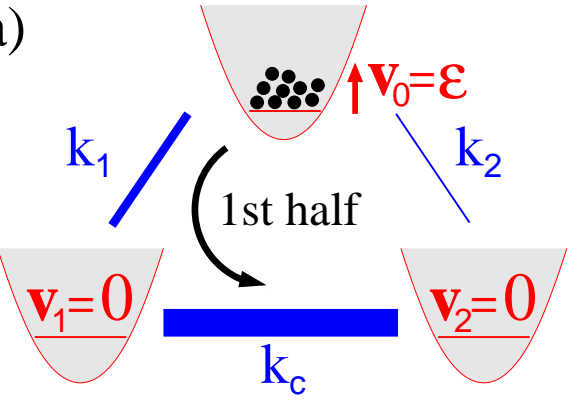

b)

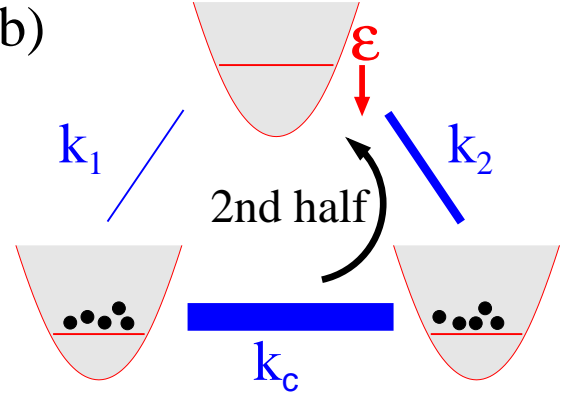

Figure 6.1.: Illustration of the model system. Initially, all particles are located on the upper site $(i=0)$ which represents the "shuttle". In the first half of the cycle (a) the on-site potential $v_{0}=\varepsilon$ is raised adiabatically slow from a very negative initial value and the particles are transported via the $k_{1}$ bond to the "canal" which is represented by the strongly coupled canal sites $(i=1,2)$. In the second half of the cycle (b) the bias in the coupling is inverted and the particles are transported back from the canal to the shuttle via the $k_{2}$ bond.

\subsection{Operating an atom pump in a closed system: The $\mathrm{BHH}$ trimer model}

The possibility to induce DC currents by periodic (AC) modulation of a potential is familiar from the context of electronic devices. If an open geometry is concerned, it is referred to as "quantum pumping" [200, 158, 42, 38, 2, 199], while for closed geometries we use the term "quantum stirring" [178]. Here we propose the stirring of condensed ultra-cold atoms $[175,145]$ due to the periodic variation of the on-site potentials and of the tunneling rates between adjunct confining traps. The simplest model that captures the physics of quantum stirring is the BHH trimer $[31,115,196,113,114]$ which is illustrated in Fig. 6.1. One site $(i=0)$ is regarded as a "shuttle" that transports the atoms, while the other two sites $(i=1,2)$ are regarded as a two-level "canal". The Bose-Hubbard Hamiltonian then reads ${ }^{1}$ :

$$
\begin{aligned}
\hat{H}= & \sum_{i=0}^{2} v_{i} \hat{n}_{i}+\frac{U}{2} \sum_{i=0}^{2} \hat{n}_{i}\left(\hat{n}_{i}-1\right)-k_{c}\left(\hat{b}_{1}^{\dagger} \hat{b}_{2}+\hat{b}_{2}^{\dagger} \hat{b}_{1}\right) \\
& -k_{1}\left(\hat{b}_{0}^{\dagger} \hat{b}_{1}+\hat{b}_{1}^{\dagger} \hat{b}_{0}\right)-k_{2}\left(\hat{b}_{0}^{\dagger} \hat{b}_{2}+\hat{b}_{2}^{\dagger} \hat{b}_{0}\right) .
\end{aligned}
$$

Without loss of generality we use mass units such that $\hbar=1$, and time units such that intracanal hopping amplitude is $k_{c}=1$. Below we assume for simplicity that $v_{1}=v_{2}=0$, and therefore the single particle canal levels are $\varepsilon_{ \pm}= \pm 1$ (see also Section 6.3.1). We consider $v_{0}=\varepsilon$ as one control parameter of the pumping cycle, hence the single particle shuttle level is $\varepsilon$. Below we assume that $N|U| \ll 1$.

\footnotetext{
${ }^{1}$ For better readability we choose the index of the original BHH (see Eq. (2.10)) to be $i=0,1,2$. The couplings are defined as $k_{c}=k_{12}, k_{1}=k_{01}=k_{10}, k_{2}=k_{02}=k_{20}$ and the interaction strength is assumed to be homogenous, i.e., $U_{i}=U$ for $i=0,1,2$.
} 

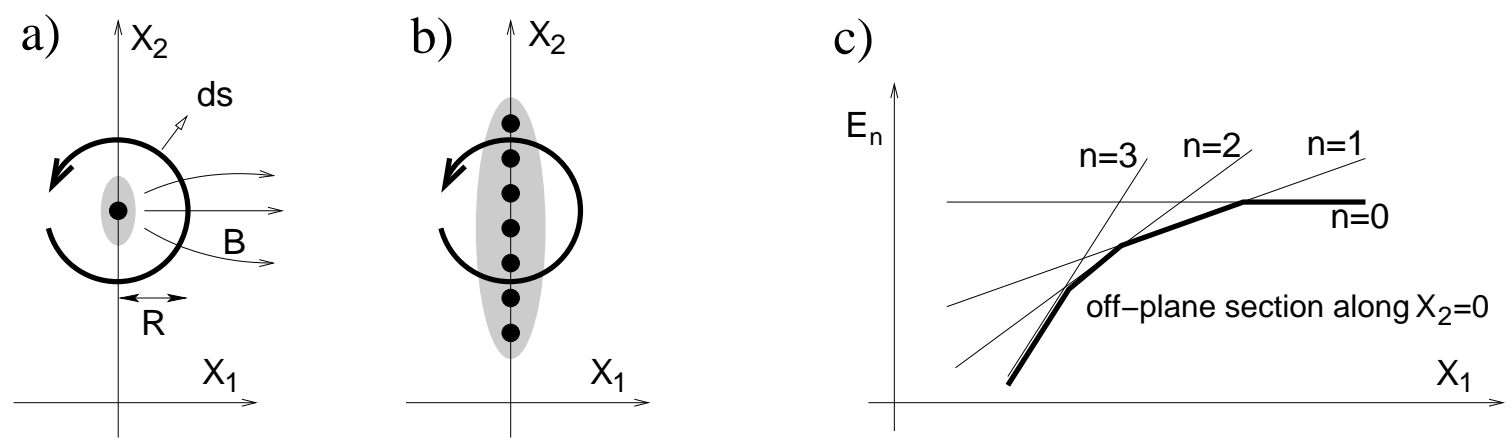

Figure 6.2.: Illustration of the pumping cycle. See the text for further details. For a large cycle that encircles the whole shaded region we have $Q \approx N$. The position of the "monopoles" is depicted by black dots: (a) no interactions (all monopoles are at the same position) (b) with interactions. In panel (c) we schematically plot the energy levels along the $X_{1}=0$ axis for a system corresponding to $N=3$ bosons.

The couplings between the shuttle and the two ends of the canal are $k_{1}$ and $k_{2}$. We assume that both are much smaller than $k_{c}$. Their inverse $1 / k_{1}$ and $1 / k_{2}$ can be regarded as barrier heights, and changing them is like switching valves on and off. It is convenient to define the two control parameters of the pumping as follows:

$$
X_{1}=\left(\frac{1}{k_{2}}-\frac{1}{k_{1}}\right), \quad X_{2}=\varepsilon
$$

By periodic cycling of the parameters $\left(X_{1}, X_{2}\right)$ we can imitate a classical peristaltic mechanism and obtain a non-zero amount $(Q)$ of transported atoms per cycle. The pumping cycle is illustrated in Figs 6.1, 6.2(a,b). Initially, all the particles are located in the shuttle which has a sufficiently negative on-site potential energy $\left(X_{2}<0\right)$. In the first half of the cycle the coupling is biased in favor of the $k_{1}$ route $\left(X_{1}>0\right)$ while $X_{2}$ is raised until, say, the shuttle is empty. Then, in the second half of the cycle, the coupling is biased in favor of the $k_{2}$ route $\left(X_{1}<0\right)$, while $X_{2}$ is lowered until the shuttle is again full. If the particles were noninteracting, i.e. assuming $U=0$, the shuttle is depopulated via the $k_{1}$ route into the lower energy level $\varepsilon_{-}$during the first half of the cycle, and re-populated via the $k_{2}$ route during the second half of the cycle. Accordingly, the net effect is to have a non-zero atom charge $Q$. If we had a single particle in the system, the net effect would be to pump roughly one particle per cycle. If we have $N$ non-interacting particles, the result of the same cycle is to pump roughly $N$ particles per cycle. We would like to know what is the actual result using a proper quantum mechanical calculation, and furthermore we would like to investigate what is the effect of the inter-atomic interaction $U$ on the result. 


\subsection{Adiabatic theory}

In analogy with Ohm's law (where $X$ is the magnetic flux, and $-\dot{X}$ is the electro motive force) the atom current is $I=-G_{1} \dot{X}_{1}$ if we change $X_{1}$ and $I=-G_{2} \dot{X}_{2}$ if we change $X_{2}$, where $G_{1}$ and $G_{2}$ are elements of the geometric conductance matrix. Accordingly

$$
Q=\oint_{\text {cycle }} I d t=-\oint\left(G_{1} d X_{1}+G_{2} d X_{2}\right) .
$$

In order to calculate the geometric conductance we use the Kubo formula approach to quantum pumping [54] which is based on the theory of adiabatic processes [25, 15, 27] (see also Appendix B for further discussion). It turns out that in the strict adiabatic limit $G$ is related to the vector field $\mathbf{B}$ (also know as "two-form") in the theory of Berry phase $[25,15,27]$. Namely, using the notations $\mathbf{B}_{1}=-G_{2}$ and $\mathbf{B}_{2}=-G_{1}$ we can rewrite Eq. (6.3) as $\left(X_{3} \equiv 0\right)$

$$
Q=\oint \mathbf{B} \cdot \vec{d} s
$$

where we define the normal vector $\overrightarrow{d s}=\left(d X_{2},-d X_{1}\right)$ as illustrated in Fig. 6.2a. The advantage of this point of view results from the intuition that it provides for the result: $Q$ is related to the flux of a field $\mathbf{B}$ which is created by "magnetic charges" in $X$ space. For $U=0$ all the "magnetic charge" is concentrated in one point. As the interaction $U$ becomes larger the "magnetic charge" disintegrates into $N$ elementary "monopoles" (see Fig. 6.2) thereby quantizing $Q$. The total charge transported during a cycle then depends on the number of monopoles that are encircled. In practice, the calculation of $\mathbf{B}$ is done using the following formula:

$$
\mathbf{B}_{j}=\sum_{n \neq n_{0}} \frac{2 \operatorname{Im}\left[I_{n_{0} n}\right] \mathcal{F}_{n n_{0}}^{j}}{\left(E_{n}-E_{n_{0}}\right)^{2}}
$$

where

$$
\hat{I}=\frac{i}{2}\left[\left(\hat{b}_{0}^{\dagger} \hat{b}_{1}-\hat{b}_{1}^{\dagger} \hat{b}_{0}\right)+\left(\hat{b}_{2}^{\dagger} \hat{b}_{0}-\hat{b}_{0}^{\dagger} \hat{b}_{2}\right)\right]
$$

is the averaged current along the bonds $0 \mapsto 1,2 \mapsto 0$ and $\hat{\mathcal{F}}^{j}=-\partial \hat{H} / \partial X_{j}$ is the generalized force (see Chapter 3.1.2) associated with the control parameter $X_{j}$. The index $n$ distinguishes the eigenstates of the many-body Hamiltonian. We assume from now on that $n_{0}$ is the BEC ground state. ${ }^{2}$

\subsection{Controlled atom current in the Bose-Hubbard trimer}

\footnotetext{
${ }^{2}$ As we have seen in Sections 2.6.3, 2.6, and 3.4, the underlying classical dynamics of the BHH can have a chaotic component for intermediate values of the energy $E=\mathcal{H}$. Here, we consider adiabatic driving of the $\mathrm{BHH}$ which is prepared in the ground state, therefore chaotic motion is not an issue.
} 


\subsubsection{Two-orbital approximation}

In order to derive explicit expressions for the conductance $G$ of the BHH trimer (6.1) we first consider the case of one particle inside the three-site system (see Fig. 6.1). As we further explain below we are later going to measure the current between the shuttle $(i=0)$ and the canal sites $(i=1,2)$ on the bonds $0 \mapsto 1$ and $2 \mapsto 0$. The model Hamiltonian and the current operators in the position basis are:

$$
\hat{H}=\left(\begin{array}{ccc}
\varepsilon & -k_{1} & -k_{2} \\
-k_{1} & 0 & -1 \\
-k_{2} & -1 & 0
\end{array}\right), \quad \hat{I}_{0 \mapsto 1}=\left(\begin{array}{ccc}
0 & -i k_{1} & 0 \\
i k_{1} & 0 & 0 \\
0 & 0 & 0
\end{array}\right), \hat{I}_{2 \mapsto 0}=\left(\begin{array}{ccc}
0 & 0 & i k_{2} \\
0 & 0 & 0 \\
-i k_{2} & 0 & 0
\end{array}\right) .
$$

It is convenient to define a shuttle-canal basis which is determined via diagonalization of the Hamiltonian with vanishing couplings to the canal $\left(k_{1}=k_{2}=0\right)$

$$
\left|S_{0}\right\rangle=\left(\begin{array}{l}
1 \\
0 \\
0
\end{array}\right),|S\rangle=\frac{1}{\sqrt{2}}\left(\begin{array}{l}
0 \\
1 \\
1
\end{array}\right),|A\rangle=\frac{1}{\sqrt{2}}\left(\begin{array}{c}
0 \\
1 \\
-1
\end{array}\right)
$$

The Hamiltonian and the current operator in the shuttle-canal basis are:

$\hat{H}=\left(\begin{array}{ccc}\varepsilon & -\bar{k} & -\Delta_{k} \\ -\bar{k} & -1 & 0 \\ -\Delta_{k} & 0 & 1\end{array}\right), \hat{I}_{0 \mapsto 1}=\frac{k_{1}}{\sqrt{2}}\left(\begin{array}{ccc}0 & -i & -i \\ i & 0 & 0 \\ i & 0 & 0\end{array}\right), \quad \hat{I}_{2 \mapsto 0}=\frac{k_{2}}{\sqrt{2}}\left(\begin{array}{ccc}0 & i & -i \\ -i & 0 & 0 \\ i & 0 & 0\end{array}\right)$,

where we defined the mean values

$$
\begin{aligned}
\bar{k} & =\frac{k_{1}+k_{2}}{\sqrt{2}} \\
\Delta_{k} & =\frac{k_{1}-k_{2}}{\sqrt{2}} .
\end{aligned}
$$

The pumping cycle starts with all particles localized in the shuttle (i.e. $\varepsilon \ll-1$ ). Therefore the adiabatic particle transport takes place during the avoided crossing of the shuttle (energy $\varepsilon$ ) and the symmetric canal state which has the lower energy $\varepsilon_{-}=-1$ (see Appendix B for a description of the transport in a two-site system). Accordingly, we focus on the upper left $(2 \times 2)$-submatrix leading to the two-orbital approximation

$$
\hat{H}^{\prime}=\left(\begin{array}{cc}
\varepsilon & -\bar{k} \\
-\bar{k} & -1
\end{array}\right), \quad \hat{I}_{0 \mapsto 1}^{\prime}=\frac{k_{1}}{\sqrt{2}}\left(\begin{array}{cc}
0 & -i \\
i & 0
\end{array}\right), \quad \hat{I}_{2 \mapsto 0}^{\prime}=\frac{k_{2}}{\sqrt{2}}\left(\begin{array}{cc}
0 & i \\
-i & 0
\end{array}\right)
$$

where dashed symbols $\left(^{\prime}\right)$ denote the reduced operators. We consider the current operator $\hat{I}$ to measure average of the currents in the $k_{1}$ and in the $k_{2}$ bonds

$$
\hat{I} \equiv \frac{1}{2}\left(\hat{I}_{0 \mapsto 1}+\hat{I}_{2 \mapsto 0}\right)=\frac{\Delta_{k}}{2}\left(\begin{array}{cc}
0 & -i \\
i & 0
\end{array}\right) .
$$


The advantage of this definition is that within the two halves of the pumping cycle $(\varepsilon$ raised/lowered) the same amount of particles is transported, i.e. it is a "symmetric" measurement. This allows us to limit the below discussion to, say, the first half of the cycle where the control parameter $\varepsilon$ is raised from a very negative initial value.

Following the same analysis as for the two-site system described in Section B.2 (starting from Eq. (B.8)) we obtain for the geometric conductance

$$
G_{2}=-\frac{\Delta_{k}}{2 \bar{k}} \frac{2 \bar{k}^{2}}{\left[(\varepsilon+1)^{2}+(2 \bar{k})^{2}\right]^{3 / 2}} .
$$

For a full cycle we get

$$
\langle Q\rangle=\oint\langle I\rangle d t=\frac{\Delta_{k}}{\bar{k}}=\frac{k_{1}-k_{2}}{k_{1}+k_{2}} .
$$

In contrast to the two-site topology, the atomic charge pumped in one cycle can be less than one, namely when the difference of the coupling strengths $\Delta_{k}$ is small compared to the mean coupling $\bar{k}$. This is the so-called near-field [190] and corresponds to a small radius $R$ of the pumping cycle (see Fig. 6.2) and happens if the monopoles (or "magnetic charges") are encircled at short distance.

\subsubsection{Evolution of energy levels}

After the preliminary considerations in the previous subsection we now turn to the analysis of the many-body problem by investigating the dynamics of the eigenenergies $E_{n}$ of the Bose-Hubbard trimer (6.1) as we change $\varepsilon$ from $\varepsilon \ll(-1)$ to $\varepsilon \gg(-1)$ while the other parameters are fixed. It is convenient to rewrite the $\mathrm{BHH}$ as

$$
\begin{aligned}
\hat{H} & =\hat{H}_{\text {shuttle }}+\hat{H}_{\text {canal }}+\hat{H}_{\text {cpl }} \\
\hat{H}_{\text {shuttle }} & =\frac{U}{2} \hat{n}_{0}(\hat{n}-1)+\varepsilon \hat{n}_{0} \\
\hat{H}_{\text {canal }} & =\frac{U}{2}\left[\hat{n}_{1}\left(\hat{n}_{1}-1\right)+\hat{n}_{2}\left(\hat{n}_{2}-1\right)\right]-\left(\hat{b}_{2}^{\dagger} \hat{b}_{1}+\hat{b}_{1}^{\dagger} \hat{b}_{2}\right) \\
\hat{H}_{\mathrm{cpl}} & =-k_{1}\left(\hat{b}_{0}^{\dagger} \hat{b}_{1}+\hat{b}_{1}^{\dagger} \hat{b}_{0}\right)-k_{2}\left(\hat{b}_{0}^{\dagger} \hat{b}_{2}+\hat{b}_{2}^{\dagger} \hat{b}_{0}\right)
\end{aligned}
$$

while the operators for the current and the generalized force are $\hat{I}_{0 \mapsto 1}=i k_{1}\left(\hat{b}_{1}^{\dagger} \hat{b}_{0}-\hat{b}_{0}^{\dagger} \hat{b}_{1}\right)$, $\hat{I}_{2 \mapsto 0}=i k_{2}\left(\hat{b}_{0}^{\dagger} \hat{b}_{2}-\hat{b}_{2}^{\dagger} \hat{b}_{0}\right)$ and $\hat{\mathcal{F}}=-\hat{n}_{0}$. In what follows we assume $0<k_{1}, k_{2} \ll k_{c}=1$, $\varepsilon \sim(-1)$, and $N|U| \ll k_{c}=1$ which allows us to apply the "two orbital approximation" described in Section 6.3.1.

We are interested in the avoided crossings of the evolving ground state with the excited states (see Eq. (6.5)). In the leading approximation of the eigenenergies $E_{n}$ the couplings $k_{1}$ and $k_{2}$ are neglected. Later we take them into account as a perturbation. For $k_{1}=k_{2}=0$ the the number $(n)$ of particles in the shuttle becomes a good quantum number. Furthermore, 


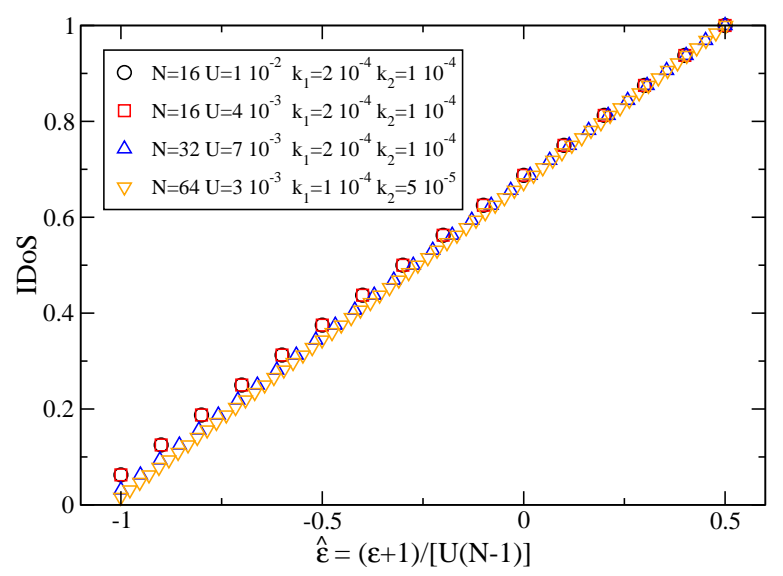

Figure 6.3.: Integrated density (IDoS) of avoided crossings ("magnetic monopoles") for various values of the parameters $U, k_{1}, k_{2}$ and the boson number $N$ as a function of the rescaled on-site potential $\hat{\varepsilon}$. The support of the IDoS is predicted by Eq. (6.25) to be $\hat{\varepsilon}=[-1,0.5]$ which is nicely confirmed.

we assume that only the lower level of the canal $\varepsilon_{-}$is occupied and that the particles are equidistributed among the canal sites. Accordingly

$$
E_{n}=E_{\text {shuttle }}(n)+E_{\text {canal }}(N-n), \quad n=0,1,2, . ., N
$$

where

$$
\begin{aligned}
E_{\text {shuttle }}(n) & =\varepsilon n+\frac{1}{2} U n(n-1) \\
E_{\text {canal }}(N-n) & =-(N-n)+\frac{1}{4} U(N-n)(N-n-1) .
\end{aligned}
$$

The degeneracy condition for the $n$-th crossing is $E_{n}-E_{n-1}=0$ leading to ${ }^{3}$

$$
\varepsilon_{n}=-1+\frac{1}{2} U \times(N-3 n+2) \quad \text { where } n=1,2, \ldots N .
$$

Accordingly, the $N$ level crossings are distributed within a range

$$
-1-(N-1) U \leq \varepsilon \leq-1+\frac{1}{2}(N-1) U .
$$

We define the rescaled version of the control variable $\varepsilon$ as

$$
\hat{\varepsilon}=\frac{\varepsilon+1}{(N-1) U}
$$

which has a support of $-1<\hat{\varepsilon}<1 / 2$. Once we take the coupling $\bar{k}$ into account, we get avoided crossings of width $\delta \varepsilon_{n}$ (see below). Within the support we should observe $N$

\footnotetext{
${ }^{3}$ Note that in $(6.23)$ the index $n$ starts at one since this corresponds to the last particle occupying the canal.
} 
such avoided crossings if they can be resolved, i.e., if their distance $U$ is large enough. In Fig. (6.3) we report the integrated density of avoided crossings (IDoS) for various values of $U, \bar{k}, \Delta_{k}$ and boson numbers $N$ that fulfill this condition. We find that all points fall in the predicted range thus the scaling (6.25) is nicely confirmed.

\subsubsection{Two-orbital approximation for the $\mathrm{BHH}$ trimer}

In complete analogy with Eq. (6.9) the Bose-Hubbard Hamiltonian in the two-orbital approximation is (say for $N=4$ ):

$$
\hat{H}=\left(\begin{array}{ccccc}
E_{0} & -\bar{k}_{1} & 0 & 0 & 0 \\
-\bar{k}_{1} & E_{1} & -\bar{k}_{2} & 0 & 0 \\
0 & -\bar{k}_{2} & E_{2} & -\bar{k}_{3} & 0 \\
0 & 0 & -\bar{k}_{3} & E_{3} & -\bar{k}_{4} \\
0 & 0 & 0 & -\bar{k}_{4} & E_{4}
\end{array}\right),
$$

where the $E_{n}$ are defined in the previous section, and the couplings are ${ }^{4}$

$$
\bar{k}_{n}=[(N+1-n) n]^{1 / 2} \bar{k} .
$$

The analogous expression applies to the current operator where $\bar{k}$ is replaced by $\Delta_{k}$. The idea of the two-orbital approximation is that the full system ground state is composed of segments. Each crossover from segment to segment in Eq. (6.26) is a (avoided) two-level crossing. For example the second crossing, i.e. from $n=3 \rightarrow n=2$, is described by

$$
\left(\begin{array}{cc}
E_{2} & -\bar{k}_{3} \\
-\bar{k}_{3} & E_{3}
\end{array}\right)
$$

which is a submatrix of Eq. (6.26). For finite $U$ and sufficiently small $\bar{k}_{n}$, we encounter a sequence of distinct avoided crossings as $\varepsilon$ is raised from $\varepsilon \ll-1$ :

$$
|4\rangle \stackrel{\bar{k}_{4}}{\longmapsto}|3\rangle \stackrel{\bar{k}_{3}}{\longmapsto}|2\rangle \stackrel{\bar{k}_{2}}{\longmapsto}|1\rangle \stackrel{\bar{k}_{1}}{\longmapsto}|0\rangle \text {. }
$$

Their $\varepsilon$-distance is of order $U$ (see Eq. (6.23)) while their width is ${ }^{5}$

$$
\delta \varepsilon_{n}=\bar{k}_{n} .
$$

The widest crossings are at the center of the $\varepsilon$-range, and they are of order $\delta \varepsilon_{n} \sim N \bar{k}$ (note that for very small interaction $U$ the width is given by the one-particle crossing width $\bar{k}$ ). This should be contrasted with the energy scales $U$ and $N U$ that describe the distance and

${ }^{4}$ Note that the calculation of $\bar{k}_{n}=\langle n-1|\hat{H}| n\rangle$ involves the matrix element $\hat{b}_{i}^{\dagger} \hat{b}_{0}$ with $i=1,2$.

${ }^{5}$ To see this we minimize the energy distance $\Delta E_{n}=\left[\left(E_{n}(\varepsilon)-E_{n-1}(\varepsilon)\right)^{2}+\left(2 \bar{k}_{n}\right)^{2}\right]^{1 / 2}$ between the actual energies taking into account the coupling. This distance $\Delta E_{n}$ is going to be small as long as the the first term in the root is smaller than the second one which is fixed with respect to $\varepsilon$. 


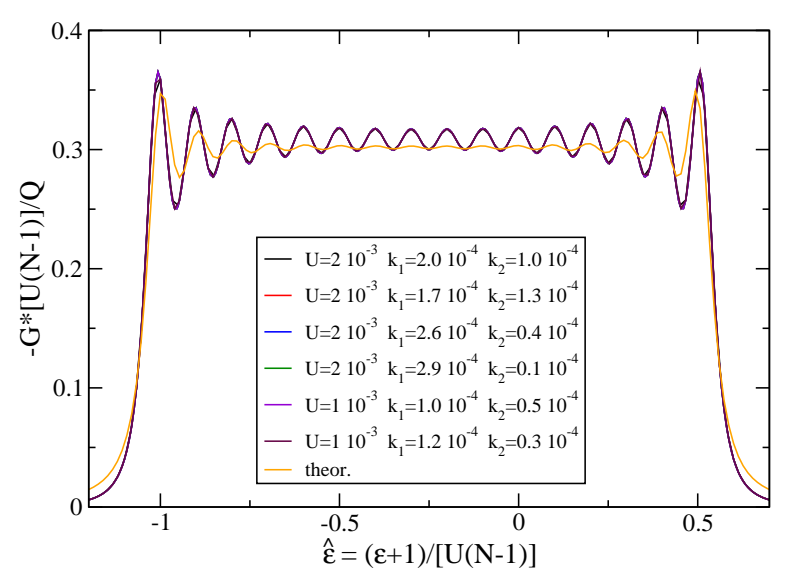

Figure 6.4.: Scaling behavior of the conductance $G$. The conductance $-G$ in the intermediate regime, for various values the coupling constants $\bar{k}, \Delta_{k}$ but constant ratio $U / \bar{k}$ and $N=16$ particles. The $x$-axis corresponds to the rescaled control variable $\hat{\varepsilon}(6.25)$ and the $y$-axis is scaled accordingly to preserve the net charge. Additionally, the ordinate was scaled by the expected charge $Q$ for a halfcycle according to Eq. (6.32) and indeed the area under the curve is $Q \approx 0.5$. One observes that the curves fall on top of one another which confirms the dependence of $G$ on the ratio $\bar{k} / U$. Additionally, we overplotted the theoretical expression (solid orange line) for the conductance (6.35). Although this expression is not expected to be valid in the intermediate regime, the agreement is pretty good. Also the agreement with the estimation from Eq. (6.36) corresponding to a constant value of $-G \approx$ 0.315 is aparent.

span of the crossings. If the widest crossings are lager than the whole span, not a single crossing will be resolved, thus we are in what we call the "mega crossing" regime. On the other hand, if even the widest avoided crossing is smaller than the spacing of the crossings $U$, i.e. $U \gg N \bar{k}$, then we are in the "sequential crossing" regime. Thus we deduce the following regimes:

$$
\begin{aligned}
U \ll \bar{k} / N & \text { mega crossing regime } \\
U \gg N \bar{k} & \text { sequential crossing regime }
\end{aligned}
$$

We observe that the behavior of the conductance depends on the ratio $\bar{k} / U$. This is confirmed in Fig. 6.4 where we plot $G_{2}$ for various $\bar{k}$ and $U$ but constant ratio. If $N$ is not too large one can resolve a sequence of two level crossings. In the following we discuss the findings in the various regimes.

\subsubsection{Regimes}

The predominant contribution to $Q$ results from the $d X_{2}$ variation $^{6}$, therefore we refer from now on to $G_{2}=G$ only. An overview of the numerical results for the conductance is shown

\footnotetext{
${ }^{6}$ If we have an rectangular pumping cycle in $X$-space, it can be closed at $X_{2}= \pm \infty$ where the influence of the change in $X_{1}$ can be safely neglected since the monopoles are located on the $X_{1}=0$ axis around $\varepsilon \approx-1$.
} 


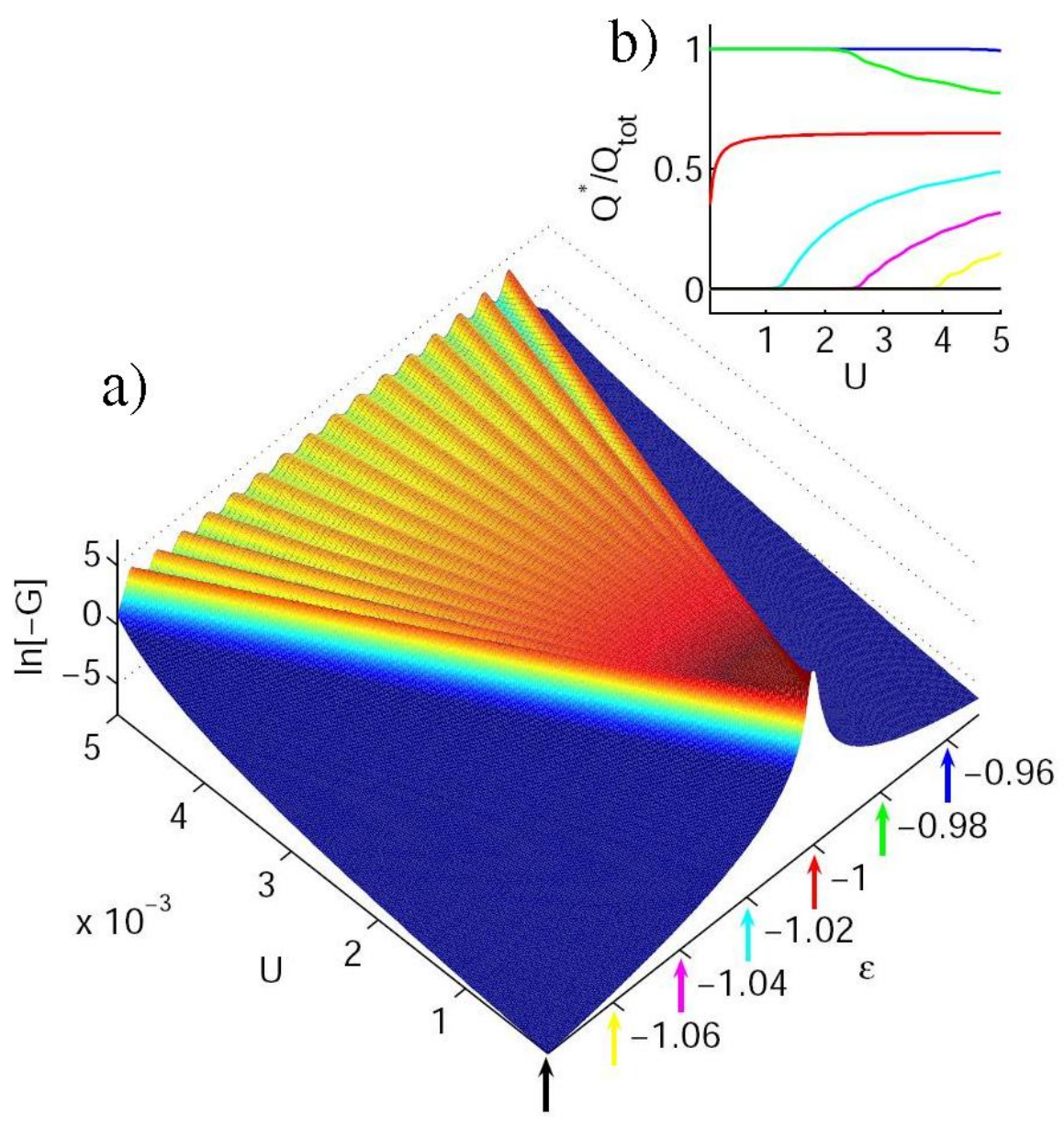

Figure 6.5.: Conductance during the first half of the pumping cycle. (a) the conductance $G_{2}$ as a function of the on-site potential $\varepsilon$, for various values of $U$. In the numerical simulations (see also Fig. 6.6) we are using exact diagonalization of the trimer Hamiltonian (6.1). For the evaluation of $G$ we use Eq. (6.5) while for $Q$ we use Eq. (6.3). The other parameters are $N=16$ particles, $\bar{k}=$ $0.0003 / \sqrt{2}$ and $\Delta k=0.0001 / \sqrt{2}$. As the interaction $U$ becomes larger one observes the crossover from a single to individual peaks in the conductance. (b), the $U$-dependence of the integrated charge $Q^{*}$, calculated for wide rectangular cycles for which $X_{2}$ is varied within $\left[-\infty, \varepsilon^{*}\right]$. The values of $\varepsilon^{*}$ are indicated by arrows of the same color in the main panel.

in Fig. 6.5, where we plot $G$ as a function of $X_{2}$ for various interaction strengths $U$. In Fig. 6.6 more details are presented: Besides $G$ we also plot the $X_{2}$-dependence of the energy levels and of the site population. Four representative values of $U$ are considered including also the case of weak attractive interactions, $U<0$.

Let us try to understand the observed results. For $U=0$ the analytical calculation is just 

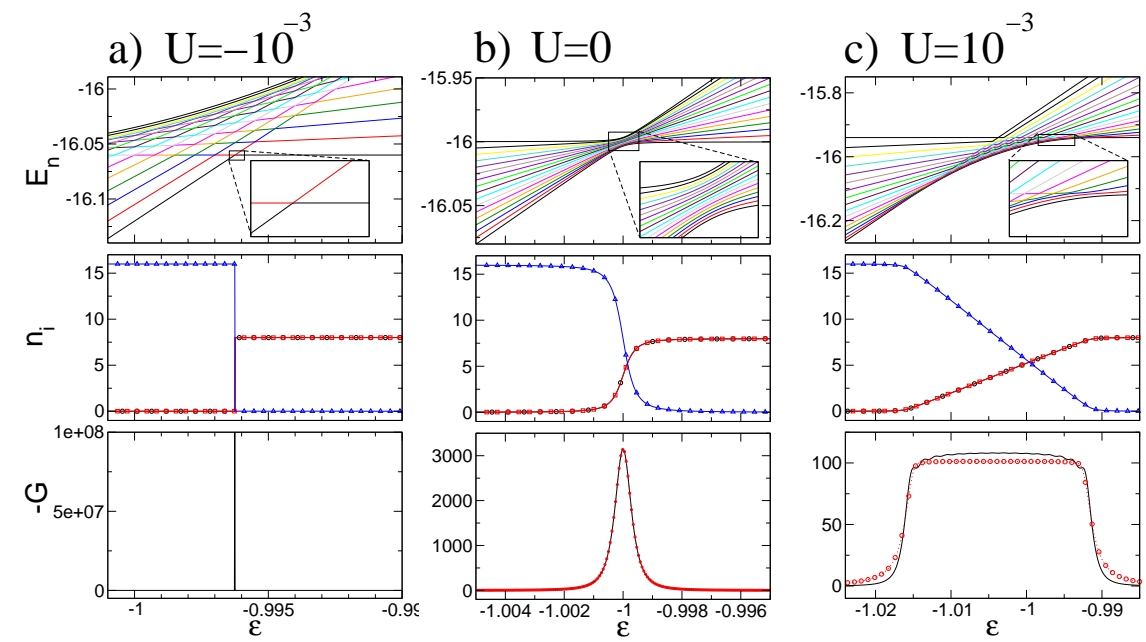

d) $\mathrm{U}=10^{-2}$

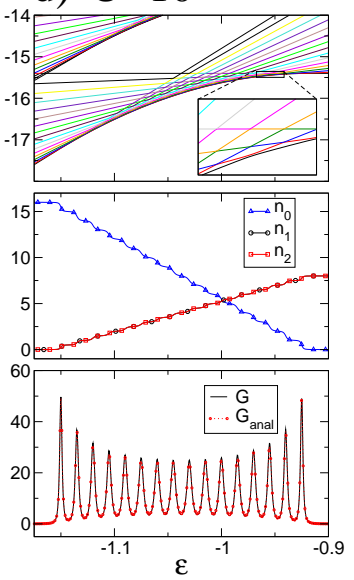

Figure 6.6.: Evolution of the energy levels, the site occupation and the conductance. Further details relating to the data of Fig. 6.5. We refer to four representative values of $U$, which are indicated on top of each set of panels. Upper panels: the lowest $N+1$ energy levels $E_{n}$ which dominate the conductance $G_{2}$ are plotted as a function of $X_{2}=\varepsilon$. The insets represent magnifications of the indicated areas. Middle panels: the site occupations $n_{0}$ (blue $\left.\Delta\right), n_{1}$ (black $\left.\circ\right), n_{2}($ red $\square)$. Note the steps of size 1 for the dot-occupation and $1 / 2$ in the wire-sites occupation in subfigure $d$. Lower panels: the corresponding conductance $G_{2}$ as a function of $\varepsilon$. Numerical results are represented by solid black lines while the dotted red line corresponds to the analytical result (6.31) in (b) and to (6.36) in (c), (d).

$N$ times the single particle result (6.14):

$$
G=-N \frac{\Delta_{k}}{2 \bar{k}} \frac{2 \bar{k}^{2}}{\left[\left(\varepsilon-\varepsilon_{-}\right)^{2}+(2 \bar{k})^{2}\right]^{3 / 2}},
$$

which can be expressed as a function of the control parameters $\left(X_{1}, X_{2}\right)$. Integrating over a full cycle one obtains

$$
Q=N \frac{\left[1+(\bar{k} R)^{2}\right]^{1 / 2}-1}{\bar{k} R},
$$

where $R$ is the radius of the pumping cycle (see Fig. 6.2a). For small cycles we get

$$
Q \approx N \bar{k} R / 2,
$$

while for large cycles we get the limiting value

$$
Q \approx N
$$

For $U=0$ and also for small values of $U$ all the particle cross "together" from the shuttle orbital to the $\varepsilon_{-}$canal orbital. We call this type of dynamics "mega crossing". 


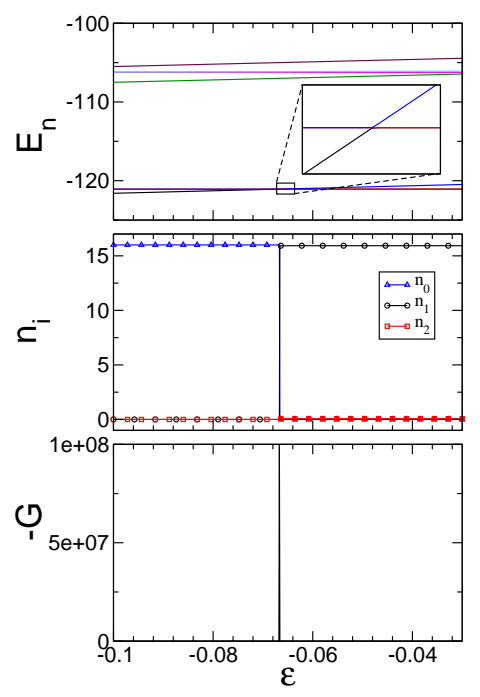

Figure 6.7.: Evolution of the energy levels, the site occupation and the conductance for strong attractive interaction $U=-1$ (see Fig. 6.6 for legend). The particles are "glued together" and roll like a classical ball from the shuttle to the left wire site as can be seen from the middle panel where the site occupations $n_{0}$ (blue $\Delta), n_{1}$ (black o), $n_{2}($ red $\square$ ) are plotted.

For very repulsive interaction $(U>0)$ we get

$$
G=-\frac{\Delta_{k}}{2 \bar{k}} \sum_{n=1}^{N} \frac{2 \delta \varepsilon_{n}^{2}}{\left[\left(\varepsilon-\varepsilon_{n}\right)^{2}+\left(2 \delta \varepsilon_{n}\right)^{2}\right]^{3 / 2}},
$$

We overplot this formula in the lower panel of Fig. 6.6d) where an excellent agreement is observed. For intermediate values of $U$ (weak repulsive interaction), namely in the range $\bar{k} \ll U \ll N \bar{k}$, we find neither the sequential crossing of Eq. (6.35), nor the mega-crossing of Eq. (6.31), but rather a gradual crossing. Namely, in this regime, over a range $\Delta X_{2}=$ $3 / 2(N-1) U$ we get a constant geometric conductance:

$$
G \approx-\frac{\Delta_{k}}{\bar{k}} \frac{1}{3 U}
$$

which reflects in a simple way the strength of the interaction. This formula has been deduced by extrapolating Eq. (6.35), and then was validated numerically (see lower panel of Fig. 6.6c).

The above scenarios can be also identified by studying the evolution of the energy levels $E_{n}$ as a function of the on-site potential $\varepsilon$. As discussed previously for large positive $U$ the $N$-fold "degeneracy" of the $U=0$ level crossing is lifted, and we get a sequence of $N$ avoided crossings (for schematic illustration see Fig. 6.2c, and compare with the numerical results in the upper panels of Fig. 6.6). Also for $U<0$ this $N$-fold "degeneracy" is lifted, but in a different way: The levels separate in the "vertical" (energy) direction rather than "horizontally" (see upper panels of Fig. 6.6). In the latter regime all the particles execute a single two-level transition from the shuttle to the canal (see Fig. 6.6a). In fact, for sufficiently strong attractive interactions $N U \ll-1$ all the particles are glued together and behave like a classical ball that rolls from the shuttle to one of the canal sites (see Fig. 6.7). When the sign of $X_{1}$ is reversed the ball rolls from one end of the canal to the other end (not 
shown). This should be clearly distinguished from the $N$-fold degenerated transition to the lower canal level which is observed in the $U=0$ case.

\subsection{Conclusions}

The theoretical $[142,147,1,216,122,73,196]$ and experimental $[50,74]$ study of driven dynamics in single and double site systems is state of the art. The study of three-site systems adds the exciting topological aspect: Controlled atomic current can be induced using optical lattice technology [3]. Our "driven vortex" should be distinguished though from the ignited stirring of $[175,145]$. The actual measurement of induced neutral currents poses a challenge to experimentalists. In fact, there are a variety of techniques that have been proposed for this purpose. For example one can exploit the Doppler effect at the perpendicular direction, which is known as the rotational frequency shift [29]. The analysis of the prototype trimer system reveals the crucial importance of interactions. The interactions are not merely a perturbation but determine the nature of the transport process. We expect the induced circulating atomic current to be extremely accurate, which would open the way to various applications, either as a new metrological standard, or as a component of a new type of quantum information or processing device. 


\section{Bose-Hubbard Dimer with Dissipation}

The interplay of intrinsic dynamics with coupling to the continuum, radiation fields or to any other external influence (such as measurement) is an important subject for various branches of modern physics: Quantum chaos [97], nanoscale devices [19], quantum optics [148], theory of decoherence and quantum computing [157] up to nuclear [146, 180, 72], atomic and molecular physics [154] are some of the areas, seemingly far remote from each other, that boosted the research in open systems. These systems are often described within the "effective non-Hermitian Hamiltonian" formalism [146]. The eigenvalues of the effective Hamiltonian are complex $\mathcal{E}_{n}=E_{n}-i \Gamma_{n} / 2$ with the non-zero imaginary part, describing the rate with which an eigenstate of the open system (termed resonance state) decays in time due to the coupling to the "outside world."

With the advent of sophisticated techniques for trapping and transporting ultracold atoms, resonance states become an issue also in the context of Bose-Einstein condensates (BEC). Examples include "atom lasers" [30], optical tweezers [105] and magnetic "conveyor belts" [109], as well as microtraps on "atom chips" [90], which have been suggested as potential building blocks for quantum information processing [184]. In this context, the aim is to understand the scattering and decay properties of a condensate leaking out of the trap via tunneling.

First theoretical investigations of the waveguide scattering of a BEC through a double barrier potential, acting like a quantum dot for the atoms, revealed intriguing nonlinear effects associated with the internal resonances of the quantum dot [162]. Complementary studies of the decay of a condensate from one open trapping potential were reported in [152], while a first attempt to study the current relaxation of an ultracold BEC, periodically driven with a standing wave of laser light, was performed in [139]. The theoretical approaches used in these studies were based on the mean field (classical) Gross-Pitaevskii equation (see Section 2.4).

However, a full quantum many-body study - using the Bose-Hubbard Hamiltonian of decaying properties of a BEC is lacking. In this context, the closed two-site system (dimer) has been analyzed thoroughly and many interesting phenomena have been found $[94,13,21,129,1,128,151,203,132,128,76,131]$. The richness of the results provides a motivation to go beyond the closed dimer and consider new scenarios where even richer behavior should be observed. In this respect, the open quantum dimer promises new and exciting opportunities, since the coupling to the continuum leads to an interesting interplay with the internal dynamics.

In this chapter we address such a scenario and study the resonance linewidths $\Gamma$ of a BHH coupled to the continuum at one of the two sites [117]. This setup can be realized using two 
coupled bosonic traps where tunneling to the continuum is imposed on one site. Another motivation arises from exciton transfer in molecular aggregates in which guest molecules (traps) are introduced interstitially and excitons are captured once they appear inside the sphere of influence of the traps [17].

This chapter is structured as follows: In the first two sections we introduce the quantum dimer model based on the effective Hamiltonian formalism and the corresponding classical Hamiltonian based on the discrete nonlinear Schrödinger (DNLS) equation. In Section 7.3, we report our results. We find the appearance of consequent bifurcations in the resonance widths $\Gamma_{n}$ as we change the on-site interaction strength $U$ which we analyze both quantum mechanically and classically. The chapter ends with a conclusion.

\subsection{Effective Hamiltonian modeling}

A usual way to treat the coupling of bound states with the continuum is to exclude the continuum degrees of freedom from the consideration by the introduction of an effective Hamiltonian $H^{\text {eff }}$ acting within the subspace of bound states and implicitly taking into account their interaction with the continuum [146]. In the case of a dimer trap, it is natural to assume that the probability to escape to the continuum is proportional to the number of the bosons located at the site (say the second one) which is coupled to the continuum with strength $\gamma$. Following this line of argumentation and using the definition of the BHH (see Eq. 2.10) with $f=2$ and on-site potentials $v_{1,2}=0$, we arrive to $H^{\text {eff }}$ given by

$$
\hat{H}^{\mathrm{eff}}=\frac{U}{2} \sum_{i=1}^{2} \hat{n}_{i}\left(\hat{n}_{i}-1\right)-k \sum_{i \neq j} \hat{b}_{i}^{\dagger} \hat{b}_{j}-i \gamma \hat{b}_{2}^{\dagger} \hat{b}_{2} .
$$

We note that a similar effective Hamiltonian was used to study a leaking dimer [135] in the presence of pumping or a driving light field [194]. The Hamiltonian (7.1) in the fixed (instantaneous) basis of the eigenstates of $\hat{N}=\sum_{i=1}^{2} \hat{n}_{i}$ reads

$$
H_{n m}^{\mathrm{eff}}=\left\{\begin{array}{cl}
\frac{U}{2}\left[n^{2}+(N-n)^{2}-N\right]-i \gamma(N-n) & n=m, \\
-k \sqrt{n(N+1-n)} & n=m+1, \\
-k \sqrt{(n+1)(N-n)} & n=m-1, \\
0 & \text { elsewhere }
\end{array}\right.
$$

where $n, m=0,1, \ldots, N$. We are interested in its (instantaneous) complex eigenvalues $\mathcal{E}_{n}=$ $E_{n}-i \frac{\Gamma_{n}}{2}$, where $E_{n}$ and $\Gamma_{n}$ are the position and width of the resonances, respectively. The subindex $n$ indicates the number of bosons on the first (non-dissipative) site. Accordingly, $N-n$ bosons are on the second (dissipative) site. The rank of $H^{\text {eff }}$ is related to the number of particles as $\mathcal{N}=N+1$ (see also Eq. 2.38). In our numerical analysis we consider traps with particle numbers $N=10$ up to 500 .

In order to gain some insight into the parametric evolution of resonance widths, we first consider the two limiting cases, vanishing interaction $U=0$ and very strong interaction 
$U \gg k$. In the former case one finds that the eigenvalues $\mathcal{E}_{n}$ of the effective Hamiltonian are given by

$$
\mathcal{E}_{n}=N \gamma\left(-\frac{i}{2}+\left(1-2 \frac{n}{N}\right) \sqrt{\Lambda^{2}-\frac{1}{4}}\right),
$$

where $\Lambda \equiv k / \gamma$ is a dimensionless ratio. From this expression it follows that for $\Lambda \geq 1 / 2$ the resonance widths are all degenerate and equal to $N \gamma$ (see Fig. 7.1a,b). In contrast, for $\Lambda<1 / 2$ the eigenvalues $\mathcal{E}_{n}$ are imaginary and we do not have any degeneracies for $U=0$ (see Fig. 7.1c). We have checked that whenever the widths $\Gamma$ show degeneracy then the corresponding positions of resonances $E_{n}$ are not degenerate, exhibiting a "dual" behavior as far as bifurcation points are concerned.

In the opposite limit of $U \gg 1$ one can neglect the inter-site hopping term and thus, the Hamiltonian (7.1) becomes diagonal leading to

$$
\mathcal{E}_{n}=N \gamma\left(\frac{U}{2 N \gamma}\left[n^{2}+(N-n)^{2}-N\right]-i\left(1-\frac{n}{N}\right)\right) .
$$

Therefore, we obtain $N$ equidistant resonance widths $\Gamma_{n}=2 \gamma(N-n)$ corresponding to $n$ particles being trapped on the first site (which resembles the resonance trapping phenomena known for the non-interacting particles [193]). Naively, one could think that the initial degeneracy of the resonance widths for $\Lambda \geq 1 / 2$ can be lifted completely by arbitrary small interaction $U$. Thus the resonance widths $\Gamma_{n}$ would flow continuously to their limiting values by increasing $U$. Below we will see that surprisingly enough this is not the case and the degeneracy is reduced each time by two at $N / 2$ discrete points. ${ }^{1}$ In Fig. 7.1 we report the resonance widths for three representative values of the coupling ratio $\Lambda=1,0.5$, and 0.4 , as a function of the interaction strength. Our numerical data are rescaled according to

$$
\tilde{\Gamma}(k, \gamma, N) \equiv \frac{\Lambda}{N k} \Gamma=\Phi_{\Lambda}(\tilde{\mathcal{U}}), \quad \tilde{\mathcal{U}}=U N / k
$$

The points corresponding to the same value of $\Lambda$ (but different values of $k$ ) fall onto the same smooth curve thus confirming (7.5). In the same figure we report the asymptotic values given by Eqs. (7.3), (7.4).

\subsection{Classical modeling: DNLS with a sink}

In order to shed some light on the emergence of the bifurcations and the scaling ansatz (7.5) we adopt a semiclassical point of view for Hamiltonian (7.1), justified in the limit of $N \gg 1$. Namely, we consider the discrete nonlinear Schrödinger (DNLS) equation with a sink [17] ${ }^{2}$

$$
\mathcal{H}=\frac{U}{2}\left[\left|A_{1}\right|^{4}+\left|A_{2}\right|^{4}\right]-k\left(A_{1}^{*} A_{2}+A_{1} A_{2}^{*}\right)-i \gamma A_{2}^{*} A_{2},
$$

\footnotetext{
${ }^{1}$ For odd numbers of bosons, there are $(N+1) / 2$ bifurcation points.

${ }^{2}$ Note that for $\gamma=0$ this leads to the standard DNLS introduced in Subsection 2.3.1.
} 


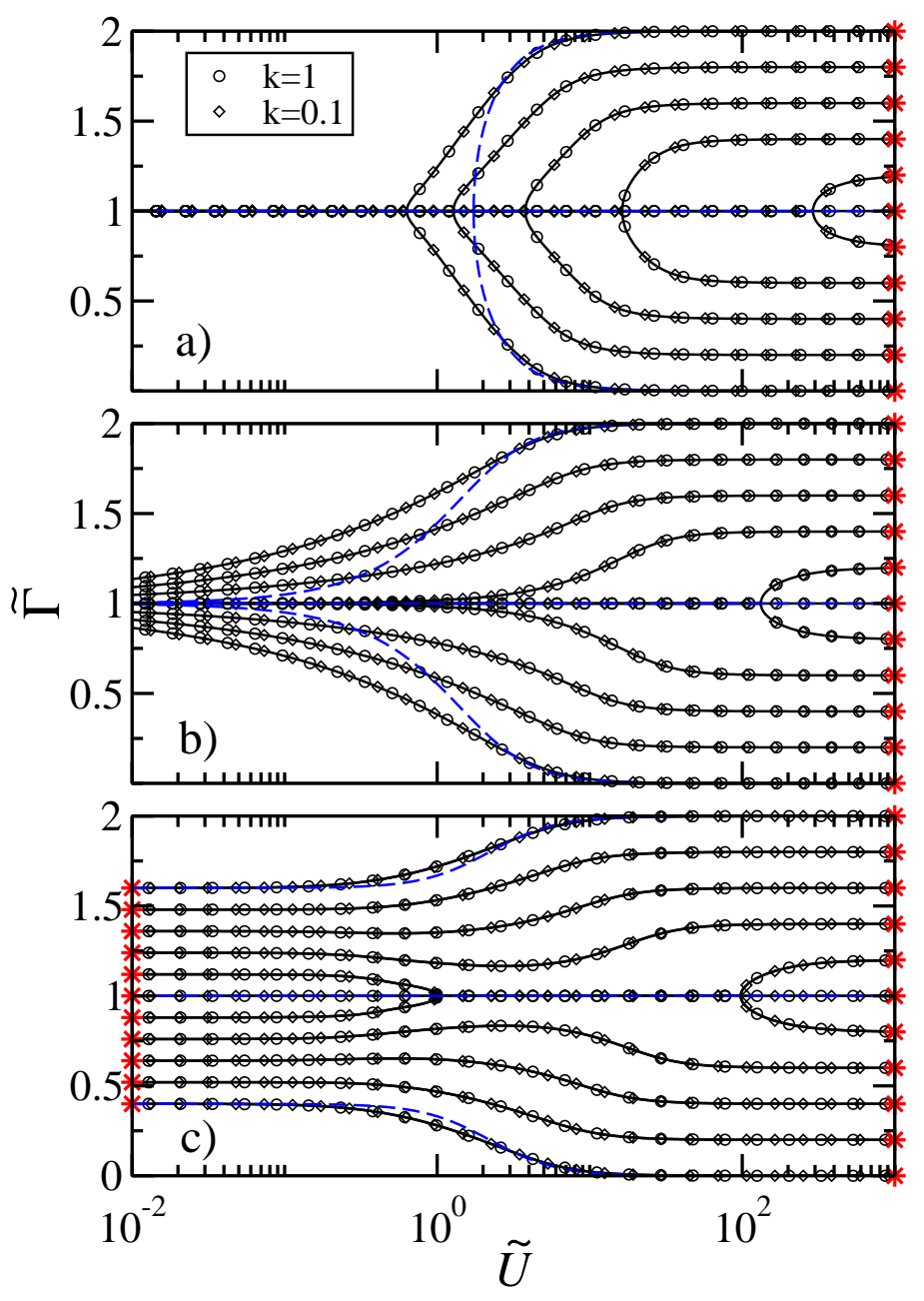

Figure 7.1.: Bifurcation diagram for the rescaled resonance widths $\tilde{\Gamma}$, vs. the interaction strength $\tilde{\mathcal{U}}$ for three representative coupling ratios: (a) $\Lambda=1$, (b) $\Lambda=0.5$, and (c) $\Lambda=0.4$. Circles (o) and diamonds $(\diamond)$ correspond to $k=1$ and 0.1 respectively. In this particular example we consider $N=$ 10. The solid lines are drawn for the eye and show the flow of resonance widths as $\tilde{\mathcal{U}}$ is changing. The stars $(\star)$ indicate the predicted asymptotic values of the resonance widths (see Eq. $(7.3,7.4)$ ). The dashed (blue) line is the "classical" result $\Gamma_{\mathrm{cl}}^{ \pm}$.

The corresponding classical eigenvalue problem reads

$$
\begin{aligned}
& \mathcal{E} A_{1}=U\left|A_{1}\right|^{2} A_{1}-k A_{2}, \\
& \mathcal{E} A_{2}=U\left|A_{2}\right|^{2} A_{2}-k A_{1}-i \gamma A_{2},
\end{aligned}
$$

which can be solved exactly. We find that for

$$
\tilde{\mathcal{U}}<\tilde{\mathcal{U}}_{\mathrm{cr}}=\sqrt{4-\Lambda^{-2}}
$$




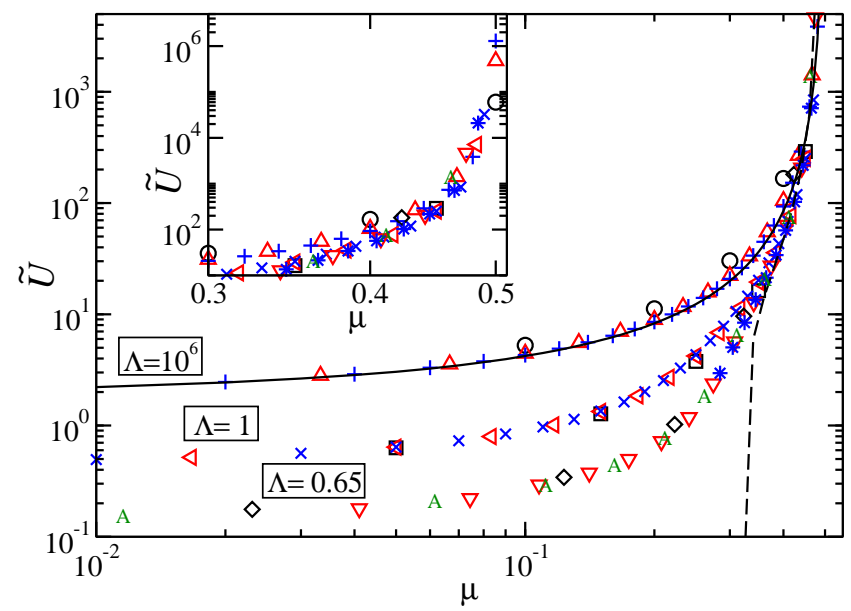

Figure 7.2.: The bifurcation points $\tilde{\mathcal{U}}_{m}$ plotted against the scaled index $\mu$ (see Eq. 7.11) for representative values of the dimensionless ratio $\Lambda=0.65,1,10^{6}$. The symbols correspond to different boson numbers $N$ and different hopping amplitudes $k: N=10, k=1$ (black), $N=30, k=10$ (red), and $N=50, k=20$ (blue). The symbol A corresponds to $N=20, k=0.1$. The solid line indicates the large $N$ limit of the case $\Lambda=10^{6}$, while the dashed line corresponds to the value $\Lambda=0.5$ and a system size of $N=50$. In the inset we show a magnification of the main figure.

there are two "trivial" solutions with a constant ( $U$-independent) value of $\Gamma=N \gamma \rightarrow \tilde{\Gamma}=1$. This corresponds to the particles being equidistributed among the two sites, i.e. $\left|A_{1}\right|^{2}=$ $\left|A_{2}\right|^{2}=N / 2$. For $\tilde{\mathcal{U}}>\tilde{\mathcal{U}}_{\text {cr }}$ two new nontrivial solutions appear with

$$
\Gamma_{\mathrm{cl}}^{ \pm}=\gamma N\left(1 \pm \sqrt{1-4 /\left(\tilde{\mathcal{U}}^{2}+\Lambda^{-2}\right)}\right)
$$

corresponding to the non-equal occupations

$$
\left|A_{1}^{ \pm}\right|^{2}=\frac{N}{2}\left(1 \mp \sqrt{1-4 /\left(\tilde{\mathcal{U}}{ }^{2}+\Lambda^{-2}\right)}\right) .
$$

The classical results are presented also in Fig. 7.1 (see dashed lines). This pitchfork bifurcation is the classical analog of the quantum bifurcations discussed above.

\subsection{Resonance widths of the $\mathrm{BHH}$ dimer}

We find that for $\Lambda \geq \Lambda^{*}=0.5$ all resonances are initially degenerate, as predicted by Eq.(7.3). As $\tilde{\mathcal{U}}$ increases, this degeneracy is lifted by consequent bifurcations. In contrary, for $\Lambda \leq 0.5$ we do not observe any initial degeneracy. We note that in the regime $\Lambda<0.5$ the instantaneous approximation is questionable since the hopping time $\tau_{k} \sim 1 / k$ is large compared to the characteristic decay time $\tau_{\gamma} \sim 1 / \gamma$. In the rest of the section we will therefore concentrate on the opposite case $\Lambda>0.5$ and analyze the sequences of bifurcation 

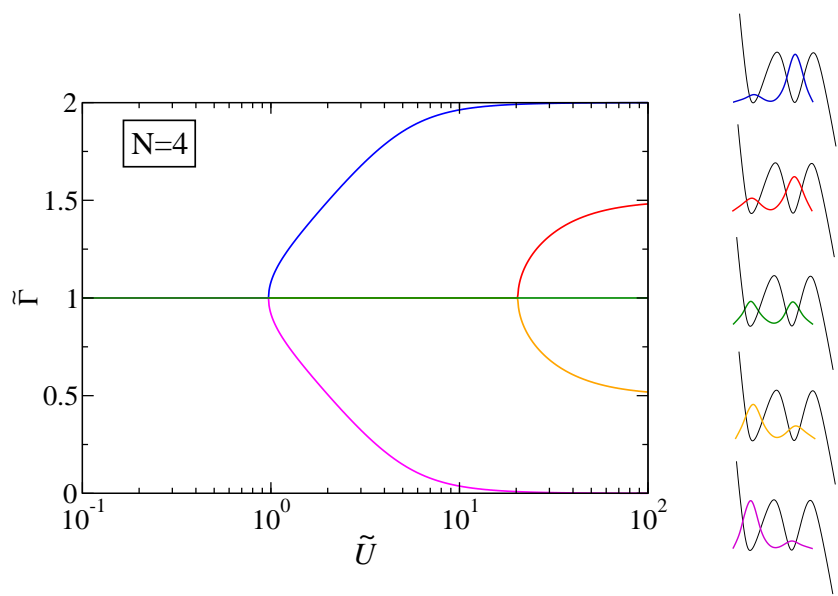

Figure 7.3.: Illustration of the bifurcations in resonance widths $\tilde{\Gamma}_{n}$ (main figure) and the associated site occupation. On the right hand side, the dimer with a sink is schematically plotted as a black line. The boson configurations associated with the resonance widths $\Gamma_{n}$ are sketched in the corresponding color, e.g. the blue curve represents a state mainly localized on the site that is coupled to the continuum while the green curve corresponds to equidistribution of particles.

points $\tilde{\mathcal{U}}_{m}, m=1, \ldots, N / 2$ which we have extracted from our numerical simulations. They were identified as the points after which two degenerate resonance widths $\tilde{\Gamma}$ differ by more than $C / N$, where we used $C=0.01$. In Fig. 7.2 we report our findings which are scaled according to

$$
\tilde{\mathcal{U}}_{m}=f_{\Lambda}(\mu), \quad \mu \equiv \frac{m-0.5 / \Lambda}{N},
$$

where the $f_{\Lambda}$ represent a set of one-parameter scaling functions which depend on $\Lambda$. An excellent agreement is evident. We have checked that our results remain qualitatively the same if we apply other similar criteria. A side remark is that the resonance positions $E_{n}$ (not shown) bifurcate at the same points $\tilde{\mathcal{U}}_{m}$.

Now we focus on the dependence of the scaling function $f_{\Lambda}$ on the parameter $\Lambda$. Our study has revealed the existence of a sort of transition separating two regimes characterized by different scaling properties. Namely, we found that at large $\mu$-values (i.e. large $\tilde{\mathcal{U}}$ ) the various $\Lambda$-curves fall nicely one on top of another, even for $\Lambda=\Lambda^{*}$, as is clearly demonstrated in the inset of Fig. 7.2. From our data we were able to estimate that this scaling of the upper part of the spectrum holds for $\mu \geq 0.35$. Notice that in the limit of $N \rightarrow \infty$ at the fixed ratio $m / N$ the scaling parameter becomes $\mu \approx m / N$, i.e. it is independent of $\Lambda$. In other words, the bifurcations corresponding to large $m$ values take place between states that are not affected by the inter-site coupling $k$ and the coupling to the continuum $\gamma$. Indeed these states correspond to configurations where the bosons are almost equidistributed among the two traps. This is also illustrated in Fig. 7.3 for the case of $N=4$ bosons.

On the other hand, for $\mu \leq 0.3$ we see that all curves, corresponding to different values of $\Lambda$ have the same functional form, albeit being shifted downwards with respect to one 


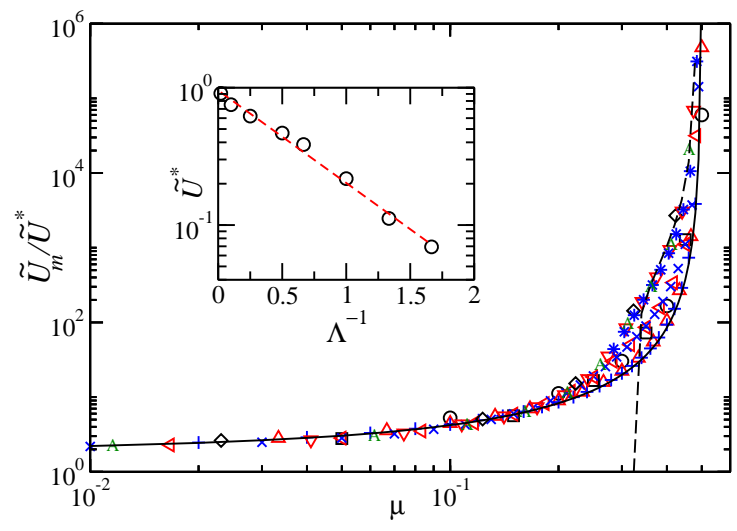

Figure 7.4.: The rescaled bifurcation points $\tilde{\mathfrak{U}}_{m} / \tilde{\mathcal{U}}^{*}$ for three values of $\Lambda=0.65,1,10^{6}$. The symbols correspond to different boson numbers $N$ and different hopping amplitudes $k$ (see Fig. 7.2). The solid line indicates the large $N$ limit of the case $\Lambda=10^{6}$. Inset: the extracted scaling parameter $\tilde{\mathcal{U}}^{*}$ as a function of the dimensionless ratio $\Lambda^{-1}$.

another (Fig. 7.2). The curves do coincide, however, when rescaling the bifurcation spectra as $\tilde{\mathcal{U}}_{m} / \tilde{\mathcal{U}}^{*}$ where $\tilde{\mathcal{U}}^{*}$ is a scaling parameter (Fig. 7.4, main part). We find that the scaling parameter $\tilde{\mathcal{U}}^{*}$ depends only on $\Lambda$ (Fig. 7.4, inset) resulting in a universal one-parameter scaling for the lower part of bifurcation points according to

$$
\tilde{\mathcal{U}}_{m} / \tilde{\mathcal{U}}^{*}=g(\mu), \quad \tilde{\mathcal{U}}^{*}=\exp (-1.55 / \Lambda),
$$

where $g(\cdot)$ is a universal ( $\Lambda$-independent) scaling function and the exponent is obtained from a best fit. We note that the scaling region becomes increasingly small as $\Lambda \rightarrow \Lambda^{*}$ and breaks down totally at $\Lambda=\Lambda^{*}$. This is the limit where the resonance widths do not show any degeneracy for $U=0$.

\subsection{Conclusions}

In conclusion we have studied the dependence of resonance widths of an open dimer BHH system on the strength of the on-site interaction and found a rich bifurcation behavior. Our results have immediate implications on the dynamics of an open dimer. Specifically, we expect that the decay of the bosons is not given by a simple exponential as suggested by the standard rate equation $\dot{N}=-\Gamma N$ with $\Gamma \sim \gamma$ being a constant. Instead, our analysis indicates a more complicated decay since now $\Gamma$ depends on the remaining particles inside the trap (see Eq. (7.5) and Fig. 7.1) and thus one has to solve a nonlinear rate equation. However, the analysis of dynamics of an open BHH system is beyond the scope of this work and is open for investigation. 


\section{Conclusions}

In this thesis, we studied interacting bosons on small lattices consisting of a few sites. The main focus was on their response to external driving fields, their transport and decay properties. This study allowed us, on the one hand, to address fundamental questions of quantum classical correspondence and, on the other hand, to investigate the implications of interactions in the transport properties of (ultra-)cold atom devices; the latter being suggested as building blocks of the emerging technology of atomtronics. Both the extraordinary degree of experimental control and precision in preparation, measurement, and inter-particle interaction, as well as the existence of a well-defined classical limit, make these systems an ideal framework to address such issues. Quantum mechanically, we described bosons on lattices using a parametric Bose-Hubbard Hamiltonian, the simplest non-trivial quantum model that takes into account the competition between the interaction energy and the kinetic energy of the system, while the corresponding classical limit is described by the discrete nonlinear Schrödinger equation.

In the first part of this thesis, we investigated the energy spreading of the bosonic system caused by three different driving schemes of the coupling strength between neighboring sites, namely the LDoS ("sudden case"), the wave-packet dynamics, and the fidelity which is a measure for quantum irreversibility. We found that in general one has to differentiate between three parametric regimes: (a) the standard perturbative regime, where basically all probability is concentrated in the initial level and where first order perturbation theory yields a valid description; (b) the extended perturbative regime, which is associated with the appearance of a core-tail structure in the energy profile, characterized by a separation of scales (the core is of non-perturbative nature, but the tails, which dominate the variance, are still described by perturbation theory). The essential ingredient in the extended perturbation theory is the appearance of the new energy scale $\Delta_{b}$. Its existence is a direct consequence of having a short but finite correlation time in the underlying (chaotic) classical dynamics; (c) the non-perturbative regime where perturbation theory (to any order) fails. At the same time, we concluded that the semiclassical limit is contained deep in the non-perturbative regime. Instead, in the perturbative limit we were able to construct an improved random matrix theory modeling which incorporates the structured energy landscape and yields a valid description of the quantum dynamics.

We also showed that the underlying classical lattice dynamics introduces a pronounced structure in the energy landscape of the perturbation operator. This has important implications in the theory of driven systems. The most dramatic consequences were observed in the study of quantum irreversibility. In this case we found the appearance of pronounced echoes in the fidelity, persistent in the deep non-perturbative (semiclassical) regime. These 
echoes are associated with the so-called self-trapping mechanism. Making use of this effect, we were able to "engineer" the echoes by preparing the initial state at a specific energy. We anticipate that similar effects will manifest themselves in cases of persisting (e.g. sinusoidal) driving leading to anomalous (nonlinear) dissipation mechanisms. A future research line will address such issues aiming at developing a response theory which goes beyond the traditional one-photon absorption scenario.

In the second part of the thesis, we addressed the transport properties of interacting bosons in the framework of adiabatically slow driving and revealed the fundamental importance of the interactions. We proposed a BEC stirring device based on the three-site Bose-Hubbard Hamiltonian which can be regarded as the incorporation of a quantum pump in a closed system. Namely, it produces an adiabatic DC atom current in response to a cyclic change of two control parameters of the (optical) lattice confining the atoms. Using the Kubo formalism we found that the nature of the transport process depends crucially on the sign and on the strength of the interatomic interactions and distinguish between four regimes of dynamical behavior: (a) for strong repulsive interaction the particles are transported one-by-one, termed sequential crossing; (b) for weaker repulsive interaction we observed gradual crossing which (c) turns to coherent mega crossing for the case of vanishing interactions; (d) finally, for strong attractive interaction the particles are glued together and behave like a huge classical ball that rolls from site to site. We expect the induced circulating atomic current to be extremely accurate, which would open the way to various applications, either as a new metrological standard, or as a component of a new type of quantum information or processing device. Further questions include the effect of lattice geometries with complex connectivity on the atomic current and the study of fluctuations (counting statistics).

In the last part, we initiated the investigation of the decay properties of a bosonic lattice system that is coupled to the continuum on one side. By using the leaking Bose-Hubbard dimer as a prototype system, we found that the interatomic interaction leads to a rich bifurcation behavior in the resonance widths. Specifically, we showed that the decay process is much more complicated than the one dictated by the standard linear rate equation. Further efforts are needed to understand better the corresponding decay law.

The rapid experimental developments in the field of interacting bosons, together with the mounting theoretical efforts to understand their dynamical properties, hold promise for the discovery of new and exciting phenomena. It is more than certain that tomorrow's technology will rely on these achievements since the properties of (ultra-)cold atoms offer a wide range of applications. 


\section{A. Level Spacing Statistics and the Semiclassical Bandprofile}

\section{A.1. Level spacing statistics of Gaussian ensembles}

In experiments, one of the best accessible quantities of a quantum mechanical system is its energy spectrum. For the hydrogen atom, for example, it can be measured by shining white light on water steam and recording the wavelengths of the transmitted light in a spectroscopy experiment. These experiments were carried out over 100 years ago and led to the Lyman-, Balmer-, Paschen-series, etc. In 1885 Balmer found empirically an analytic expression for the wavelengths measured in the experiments. On the theoretical side, we can calculate the spectra of the hydrogen and even of the helium atom. For heavier elements (= increasing number of degrees of freedom) this task becomes unsolvable as we have to deal with non-integrable systems as the energy is the only conserved quantity. Statistical properties of energy spectra were first investigated in this context of nuclear physics. In the 1960s, Wigner, Dyson, Metha and others developed random matrix theory in order to understand the statistical properties of those spectra. What is the key idea of RMT? As the Hamiltonians of heavy nuclei are usually extremely complicated Wigner assumed that the corresponding matrix ${ }^{1} \mathbf{H}$ could be substituted by a random matrix ensemble [208, 209]. This is an ensemble of square matrices of size $n \times n$ (with the purpose of considering $n \rightarrow \infty$ ). The matrix elements $H_{n m}$ are obtained from Gaussian ensembles, thus they are independent random numbers except for some symmetry requirements leading to the concept of universality classes. The systems are then classified into those with/without time-reversal symmetry and with/without spin-1/2 interaction. In matrix mechanics this translates to an invariance under orthogonal, unitary and symplectic transformations. ${ }^{2}$ Following the idea of universality, the set of all real symmetric matrices with Gaussian distributed elements will be called Gaussian Orthogonal Ensemble (GOE). In the same way the Gaussian Unitary Ensemble (GUE) and the Gaussian Symplectic Ensemble (GSE) are constructed. Using such ensembles instead of the matrices belonging to the dynamical systems, Wigner was able to predict the correct distribution of spacings between the energy levels. At that time, nuclear physics was already a field of high activity but interest in this theory was enormously renewed with the conjecture of Bohigas, Giannoni and Schmidt (BGS) [34] which we will quote in the original:

"Spectra of time-reversal-invariant systems whose classical analogs are $\mathrm{K}$

\footnotetext{
${ }^{1}$ Matrix representation and block-diagonal form are discussed in Subsection A.1.1.

${ }^{2}$ Universality classes are discussed in Subsection A.1.2.
} 
systems show the same fluctuation properties as predicted by GOE [special case of RMT]. [...] If the conjecture happens to be true, it will then have been established the universality of the laws of level fluctuations in quantal spectra already found in nuclei [...]. Then they should also be found in other quantal systems such as molecules, hadrons, etc. “

Why do we consider this conjecture to be of great importance? Although it was originally formulated for quantum mechanical systems as nuclei, the BGS conjecture is not limited to this case but can be applied in a much more general context of quantum chaos: it implies that RMT should also be applicable to wave systems in the classical realm. To this end, a lot of experimental evidence confirms this conjecture [197].

Let us have a look at Figure A.1 where an exemplary spectral measure is shown. We immediately recognize, that all histograms follow the same black solid line. This line corresponds to the RMT-prediction for the measure shown. However, the origins of the corresponding spectra differ wildly. On the one hand, we have quantum mechanical systems as a hydrogen atom in a strong magnetic field (b) and an excited $\mathrm{NO}_{2}$ molecule (c). On the other hand, there is a mechanical (a), an acoustical (d) and a microwave billiard (e) representing classical systems. Apart from time-reversal invariance, the presence of chaos is the common property of the above-mentioned systems. This remarkable observation led to the idea that the precise form of the interaction in such systems is not relevant for the statistical properties of spectral measures. These measures are related to the matrix elements of the corresponding Hamiltonian. Due to the complexity (high order correlations) of chaotic systems the matrix entries look random. As mentioned above, the basic but very radical concept of RMT is to forget about the correlations and to use random numbers instead.

In Figure A.1 we have shown one of the most popular spectral measures, the so-called level spacing distribution. The level spacings $S_{n}$ of a given Hamiltonian are defined as

$$
S_{n}=\frac{E_{n+1}-E_{n}}{\Delta(E)}
$$

where the $E_{n}$ are the ordered eigenenergies and $\Delta(E)$ is the local mean level spacing. We have seen that the distribution $P(S)$ is the same for a large variety of systems. Thus, we have just encountered a universal feature which we can use to classify a system. Let us try to better understand the features of $P(S)$.

We could now present the (lengthy) calculations of Wigner but he himself showed a more elegant way to obtain the random matrix theory predictions. This is called Wigner's surmise and uses $2 \times 2$-matrices (!) to calculate the distribution function $P(S)$. Here we will confine ourselves to the GOE and GUE cases. Although we thereby do not obtain the exact results, our approximation is so good that the maximum deviation never exceeds two percent (see [106] for details).

Let the Hamiltonian be

$$
\mathbf{H}=\left(\begin{array}{cc}
H_{11} & H_{12} \\
H_{12}^{*} & H_{22}
\end{array}\right)=\left(\begin{array}{cc}
a+b & x+i y \\
x-i y & a-b
\end{array}\right) \quad a, b, x, y \in \mathbb{R} .
$$



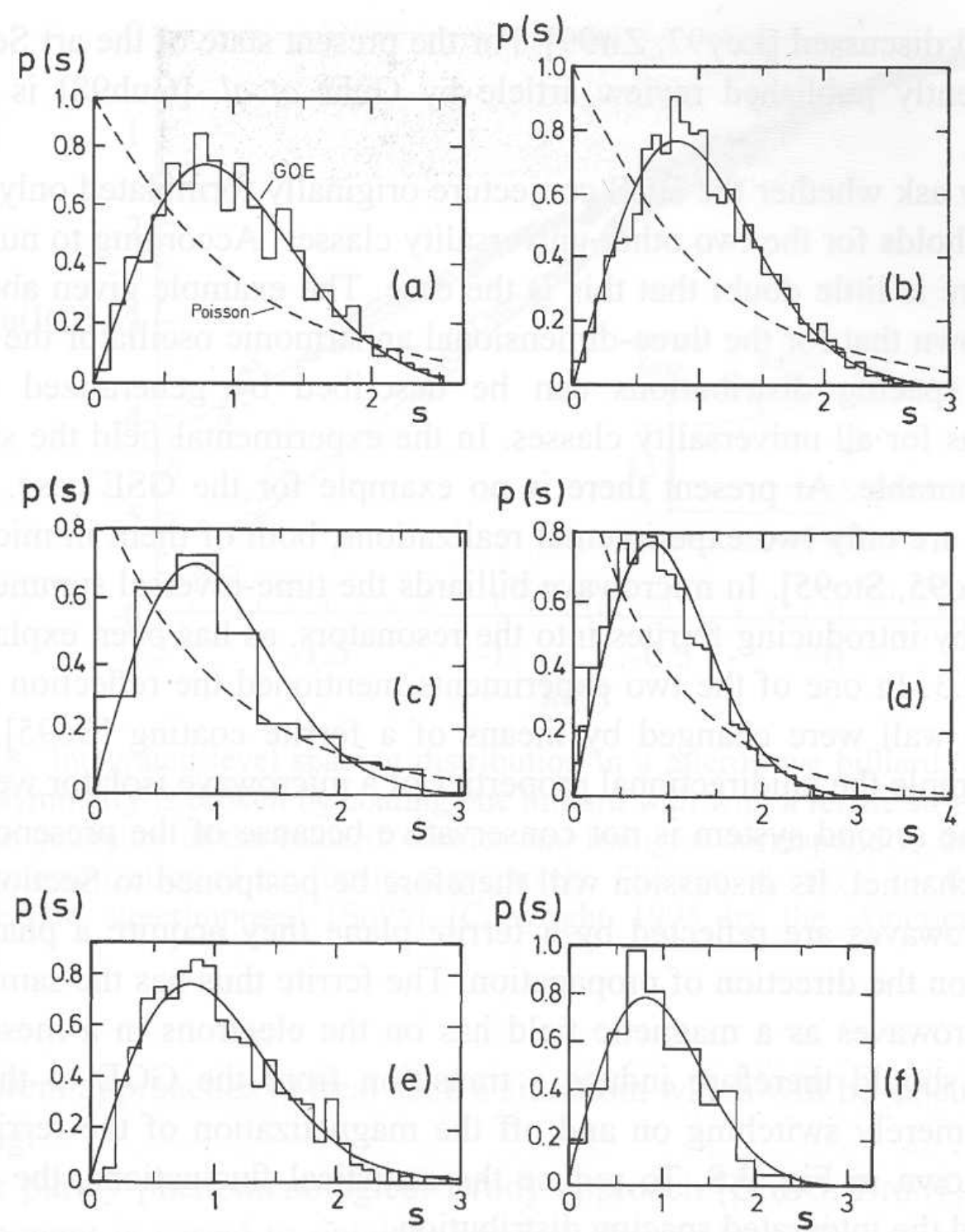

Figure A.1.: Level spacing distribution for a Sinai billiard (a), a hydrogen atom in a strong magnetic field (b), the excitation spectrum of an $\mathrm{NO}_{2}$ molecule (c), the acoustic resonance spectrum of a Sinai-shaped quartz block (d), the microwave spectrum of a three dimensional chaotic cavity (e), and the vibration spectrum of a quarter-stadium shaped plate (f). In all cases a Wigner distribution is found though only in the first three cases the spectra are quantum mechanical in origin. Taken from [197].

In the GOE the matrix $\mathbf{H}$ is real symmetric and $y$ vanishes, while for the GUE $y \neq 0$. As the number of eigenvalues is two, there is only one level spacing to be calculated

$$
S=E_{2}-E_{1}=\sqrt{b^{2}+x^{2}+y^{2}} .
$$


Assuming a Gaussian distribution $W$ for the variables $b, x$ and $y$ we obtain for the $\operatorname{GOE}(y=0)$

$$
\begin{aligned}
P(S) & =\iint_{-\infty}^{\infty} \mathrm{d} b \mathrm{~d} x W(b, x) \delta\left(S-\sqrt{b^{2}+x^{2}}\right) \\
& =2 \pi \int_{0}^{\infty} \mathrm{d} r r \frac{1}{\sqrt{2 \pi} \sigma} e^{-\frac{r^{2}}{2 \sigma^{2}}} \delta(S-r) \\
& =\frac{\sqrt{2 \pi}}{\sigma} S e^{-\frac{S^{2}}{2 \sigma^{2}}} .
\end{aligned}
$$

The same calculation for the GUE leads to

$$
\begin{aligned}
P(S) & =\iiint_{-\infty}^{\infty} \mathrm{d} b \mathrm{~d} x \mathrm{~d} y W(b, x, y) \delta\left(S-\sqrt{b^{2}+x^{2}+y^{2}}\right) \\
& =4 \pi \int_{0}^{\infty} \mathrm{d} r r^{2} \frac{1}{\sqrt{2 \pi} \sigma} e^{-\frac{r^{2}}{2 \sigma^{2}}} \delta(S-r) \\
& =\frac{\sqrt{8 \pi}}{\sigma} S^{2} e^{-\frac{S^{2}}{2 \sigma^{2}}} .
\end{aligned}
$$

The good agreement of this theory with experimental results for GOE and GUE systems is shown in Figure A.2. The system used is a two dimensional chaotic microwave billiard where time-reversal symmetry can be broken by replacing one boundary with a ferromagnetic material. Instead of the quantity $P(S)$ we present the integrated distribution $I(S)$ defined as

$$
I(S)=\int_{0}^{S} \mathrm{~d} S P(S)
$$

A clear difference is observed in the behavior of $I(S)$ for small spacings $S$ regarding the two universality classes.

For the GSE we would use a $4 \times 4$ matrix yielding the result

$$
P(S)=\frac{2^{18}}{3^{6} \pi^{3}} S^{4} e^{-\frac{64}{9 \pi} S^{2}} .
$$

Let us finally- for the sake of completeness - calculate the level spacing distribution for an integrable system. In this case the matrix representation of the corresponding Hamiltonian takes a diagonal form from symmetry considerations alone as described in Section A.1.1. As each eigenvalue corresponds to its own symmetry class, it is reasonable to assume that they are uncorrelated. In this case the probability, $P(S) \mathrm{d} S$, to find an eigenvalue in the interval $[S, S+\mathrm{d} S]$ but not in between can be easily calculated. We divide the interval $[0, S]$ 
Figure A.2.: Integrated level spacing distribution (see Eq.(A.6)) of a GOE (triangles) and a GUE (circles) system realized in a microwave experiment [192]. The theoretical curves are superimposed.

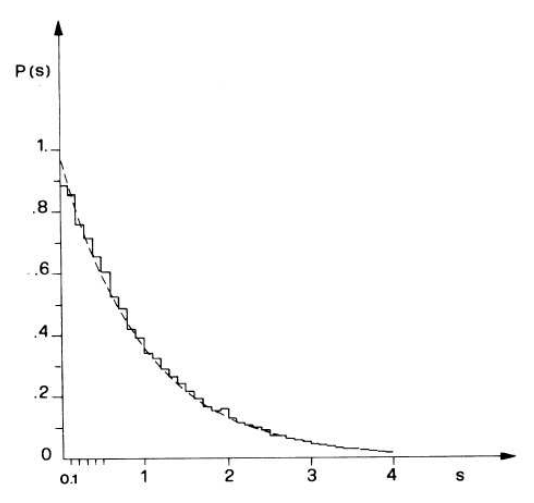

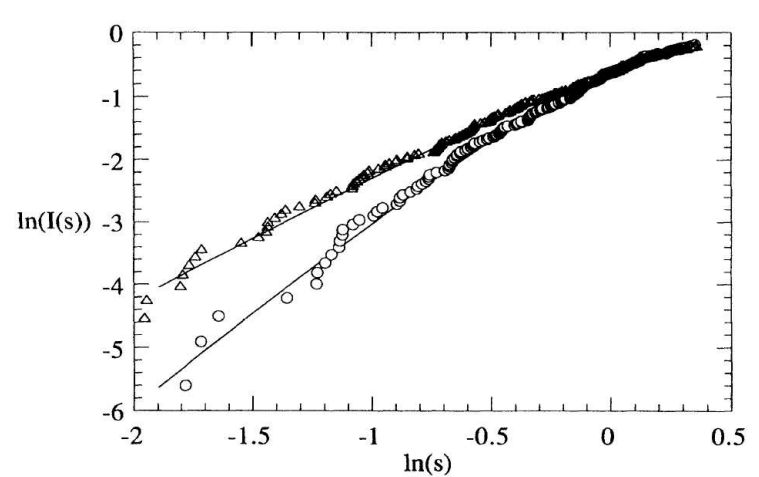

Figure A.3.: Level spacing distribution for the first 100000 levels of a rectangular billiard. The dashed line corresponds to a Poisson distribution. Figure taken from [44].

into $N$ equidistant subintervals (of length $\frac{S}{N}$ ). Since the eigenvalues are uncorrelated, the probability to find no eigenvalue in the interval $[0, S]$ can be written as $\lim _{n \rightarrow \infty}\left(1-\frac{S}{N}\right)^{N}$. The probability to find an eigenvalue in the appended interval $[S, S+\mathrm{d} S]$ is simply $\mathrm{d} S$ leading to

$$
P(S) \mathrm{d} S=\lim _{N \rightarrow \infty}\left(1-\frac{S}{N}\right)^{N} \mathrm{~d} S
$$

After performing the limit we obtain the Poisson nearest neighbor distribution

$$
P(S)=e^{-S}
$$

This distribution has been observed in a large number of integrable systems as for example rectangular billiards studied by Casati et al. [44]. Their results are presented in Figure A.3 where we can clearly see the Poissonian distribution characterized by a high probability for small spacings.

We summarize now the results for all four classes of systems: ${ }^{3}$

\footnotetext{
${ }^{3}$ The variance of the distribution $\sigma^{2}$ has been set according to the normalization of the probability.
} 


$$
P(S)=\left\{\begin{array}{cl}
S^{0} e^{-S} & \text { integrable } \\
\frac{\pi}{2} S^{1} e^{-\frac{\pi}{4} S^{2}} & G O E \\
\frac{32}{\pi^{2}} S^{2} e^{-\frac{4}{\pi} S^{2}} & G U E \\
\frac{2^{18}}{3^{6} \pi^{3}} S^{4} e^{-\frac{64}{9 \pi} S^{2}} & G S E
\end{array} .\right.
$$

What is the important difference between these distributions? First of all, we see that $P(S)$ depends strongly whether we have an integrable or a chaotic system. In the first case, small level spacings are very likely while in the latter they seem to be avoided. This effect is called level repulsion and we could have seen it already from Eq.(A.3). As mentioned before, for an integrable system the Hamilton matrix is diagonal. Therefore, the level spacing depends on only one parameter $(b)$. Due to its distribution, a spacing of $S=0$ is most likely to be observed. For GOE systems the level spacing is already a sum of two Gaussian distributed positive numbers. As they are independent, it is less probable to have $S=0$ leading to linear level repulsion. In the GUE case three independent random numbers need to vanish while for the GSE there are five. As we can see from the above expression (A.10), the degree of level repulsion is dominated by the first term of the distribution, to be more precisely by the power of $S$. The power is called the universality index $\beta$ which takes the values $0,1,2$ and 4 for the four classes respectively. The level repulsion increases as the symmetry of the matrix representing the Hamiltonian becomes more complex. In summary we can say that RMT did very well in predicting the correct level spacing distribution of complex systems.

\section{A.1.1. Symmetry and block matrix representation}

In the following many ideas are taken from the introductory book of Stöckmann [197]. Conservative systems play an important role in physics. Here, the Hamiltonian $\hat{H}$ does not depend explicitly on time and therefore the time-dependent Schrödinger equation

$$
i \hbar \frac{\partial \psi}{\partial t}=\hat{H} \psi
$$

can be transformed into the stationary Schrödinger equation

$$
\hat{H} \psi_{n}(x, t)=E_{n} \psi_{n}(x, t)
$$

where

$$
\psi_{n}(x, t)=\psi_{n}(x) e^{i E_{n} t / \hbar}
$$


are the eigenfunctions of $\hat{H}$. Although later in this work, non-conservative processes will be studied it isn't necessary to extend the theory to that case as we shall see. For a completely chaotic system the eigenenergies $E_{n}$ are the only constants of motion. We can now expand the eigenfunctions $\psi_{n}(x, t)$ in an arbitrary basis

$$
\psi_{n}(x)=\sum_{m} a_{n m} \phi_{m}(x)
$$

where the basis vectors $\phi_{n}$ satisfy the orthonormality relation

$$
\left\langle\phi_{n} \mid \phi_{m}\right\rangle=\int \phi_{n}^{*}(x) \phi_{m}(x) \mathrm{d} x=\delta_{n m} .
$$

Here, the symbols $\langle\cdot|,| \cdot\rangle$ belong to Dricac's bra-ket notation. Inserting the expansion (A.14) into the stationary Schrödinger equation (A.12) we obtain its matrix representation in the $\phi_{n}$ basis

$$
\sum_{m} a_{n m} H_{n m}=a_{n} E_{n}
$$

where

$$
\begin{aligned}
H_{n m} & =\left\langle\phi_{n}|\hat{H}| \phi_{m}\right\rangle \\
& =\int \phi_{n}^{*}(x) \hat{H} \phi_{m}(x) \mathrm{d} x .
\end{aligned}
$$

In this way the problem of solving the Schrödinger equation has been reduced to the task of diagonalizing the matrix $\mathbf{H}$.

As symmetry is a very successful concept in physics that usually simplifies the problem we shall now have a look in how far it will be of help for us. What happens to the matrix representation if the Hamiltonian is invariant under a symmetry operation $T: x \rightarrow x^{\prime}$ ? The invariance means that we can define an operator $\hat{R}$ with

$$
\hat{H}\left(x^{\prime}\right)=\hat{H}(x)+\hat{R} \hat{H}(x) \stackrel{\text { def }}{=} \mathcal{H}(x) .
$$

This can be expressed via the commutator

$$
[\hat{H}, \hat{R}]=0 .
$$

As $H$ is self-adjoint it also commutes with $\hat{R}^{\dagger}$ so that we can assume without loss of generality that $\hat{R}$ itself is self-adjoint (if not we construct it for example from $\left(\hat{R}+\hat{R}^{\dagger}\right)$ ). Let now $\phi_{n, a}$ be a set of basis vectors of $\hat{R}$

$$
\hat{R} \phi_{n, \alpha}=r_{n} \phi_{n, \alpha}
$$

where the $r_{n}$ denominate different eigenvalues and $\phi_{n, \alpha}$ the corresponding eigenfunctions. Relation (A.19) then becomes

$$
\begin{aligned}
0 & =\left\langle\phi_{n, \alpha}|\hat{H} \hat{R}-\hat{R} \hat{H}| \phi_{m, \beta}\right\rangle \\
& =\left(r_{n}-r_{m}\right)\left\langle\phi_{n, \alpha}|\hat{H}| \phi_{m, \beta}\right\rangle
\end{aligned}
$$


As all eigenvalues have been assumed to be different, the right hand side must vanish for $n \neq m$, giving

$$
\left\langle\phi_{n, \alpha}|\hat{H}| \phi_{m, \beta}\right\rangle=\delta_{n m} H_{\alpha, \beta}^{(n)}
$$

where

$$
H_{\alpha, \beta}^{(n)}=\left\langle\phi_{n, \alpha}|\hat{H}| \phi_{n, \beta}\right\rangle .
$$

With this transformation we achieved that the matrix representation of $\hat{H}$ has a block form

$$
\mathbf{H}=\left(\begin{array}{ccc}
\mathbf{H}^{(1)} & 0 & \cdots \\
0 & \mathbf{H}^{(2)} & \ldots \\
\vdots & \vdots & \ddots
\end{array}\right)
$$

and thus, the diagonalization has become less costly. For every further symmetry we can repeat this procedure until finally the matrix becomes irreducible. This is especially important for non-integrable systems (see second-next paragraph).

In classical mechanics every symmetry of the system gives rise to a constant of motion. The quantum mechanical analog are the quantum numbers. In an integrable system the number of constants of motion equals the number of degrees of freedom. This is for example the case for the hydrogen atom. The electron has three translational degrees of freedom and one spin degree of freedom. It is unambiguously described by the four quantum numbers for energy $(n)$, total angular momentum $(l)$, and the projection of angular momentum and electron spin onto the $z$-axis $\left(m, m_{s}\right)$. Atomic nuclei of higher order lack such a complete set of quantum numbers and therefore represent typical non-integrable systems. Their spectra have been studied extensively and the most famous spectral property investigated is the distribution of the energy level spacings (see Section A.1).

Non-integrable Systems As the eigenvalues of each symmetry are assumed to be uncorrelated, it is not meaningful to mix the spectra of different block matrices $\mathbf{H}^{(n)}$ of (A.24) and then to calculate the level spacing distribution. How superimposing different spectra can affect the statistics is shown in Figure A.4, where the remaining symmetry is gradually removed following the subfigures from top to bottom. Therefore, we assume from now on, that the Hamiltonian $\hat{H}$ belongs to only one set of quantum numbers resp. to one symmetry, i.e., its matrix representation is irreducible. Still, there is another symmetry that has not been treated up to now which is the symmetry with respect to time.

\section{A.1.2. Universality classes}

The time inversion operator $\hat{T}_{\text {inv }}$ changes the sign of time $t$

$$
\hat{T}_{\text {inv }} f(t)=f(-t) .
$$




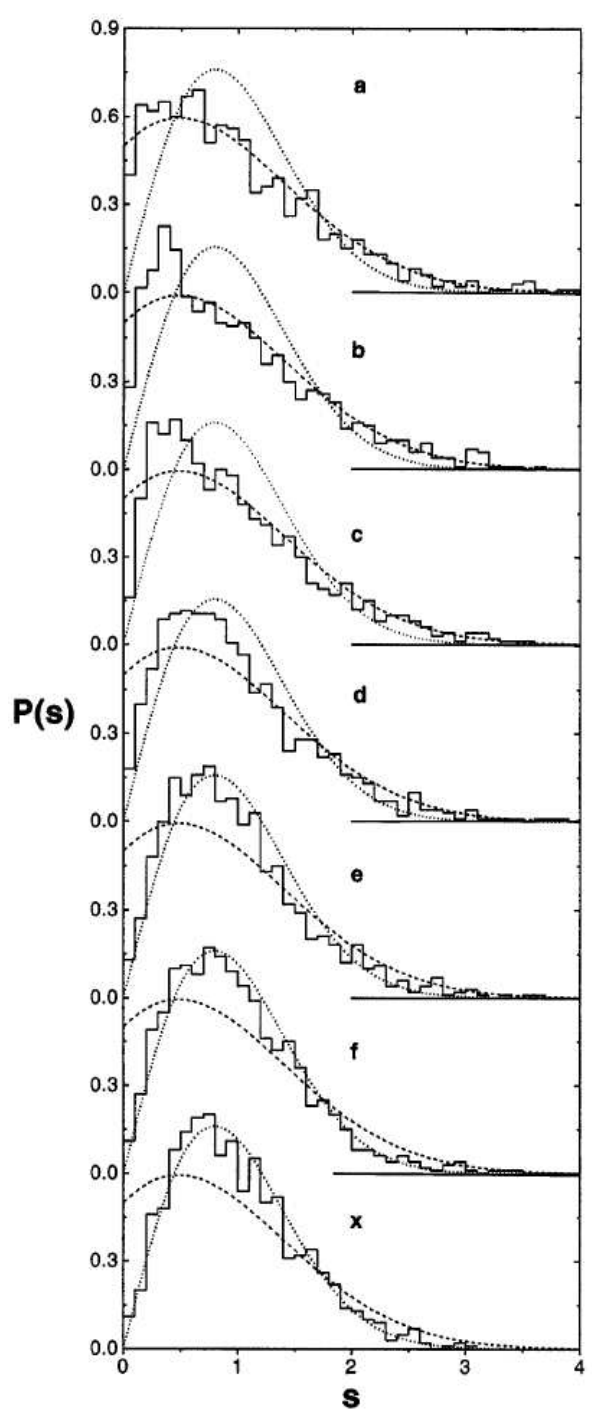

Figure A.4.: Level spacing distribution of the acoustic resonances of a quartz block. An octant of a sphere has been removed from one corner with increasing radius (a) $r=0 \mathrm{~mm}, \mathrm{~b}$ ) $r=0.5 \mathrm{~mm}$, c) $r=0.8 \mathrm{~mm}$, d) $r=1.1 \mathrm{~mm}$, e) $r=1.4 \mathrm{~mm}$, f) $r=$ $1.7 \mathrm{~mm}, \mathrm{x}) r=10 \mathrm{~mm}$ ). The dashed line corresponds to the theoretical prediction of the distribution for a system with a remaining symmetry while the dotted line applies to a system without an additional symmetry. Taken from [80].

On the left hand side of the time-dependent Schrödiger equation (A.11) this can be compensated by changing $i$ to $-i$, i.e., applying the conjugate-complex operator $\hat{C}$. In terms of commutators this reads

$$
\left[\hat{C} \hat{T}_{\mathrm{inv}}, i \hbar \partial_{t}\right]=0
$$

Does $\hat{H}$ on the right hand side also commute with $\hat{C} \hat{T}_{\text {inv }}$ ? As we have restricted ourselves to conservative systems, $\hat{H}$ commutes with the time-reversal operator thus reducing the problem to the commutator

$$
[\hat{C}, \hat{H}]=? .
$$

At this point, three situations are possible, leading to the concept of universality classes. 
Let us first consider a Hamiltonian with a non-vanishing vector potential $\hat{\mathbf{A}}$

$$
\mathcal{H}=\frac{1}{2 m}\left(\hat{\mathbf{p}}-\frac{e}{c} \hat{\mathbf{A}}\right)+\hat{V}(x) .
$$

The commutator (A.21) differs from zero because $\hat{\mathbf{p}}$ is a complex operator. For this type of systems the time-reversal symmetry is broken, say by a magnetic field. Nevertheless, $\hat{\mathcal{H}}$ is Hermitian leading to

$$
H_{n m}=H_{m n}^{*} .
$$

Therefore the matrix elements of $\mathbf{H}$ will in general be complex. The Hermiticity of such a matrix is respected under unitary transformations

$$
\mathbf{H}^{\prime}=\mathbf{U H} \mathbf{U}^{\dagger},
$$

where $\mathbf{U U}^{\dagger}=$ Id. This will then lead to the notion of unitary ensemble.

The next two possibilities represent systems with time-reversal symmetry but we have to distinguish between Hamiltonians with or without spin-1/2 interaction. In the first case the Hamiltonian reads

$$
\hat{H}=\frac{\hbar^{2}}{2 m} \hat{p}^{2}+\hat{V}(x) \text {. }
$$

Here the commutator (A.27) vanishes and both operators can be diagonalized in the same basis. As $\hat{C}^{2}=$ Id the eigenvalues of $\hat{C}$ are \pm 1 . Without loss of generality we choose the eigenfunctions of $\hat{C}$ (and therefore also the basis functions $\phi_{n}$ of $\hat{H}$ ) to be real. This implies that also the matrix elements of $\mathbf{H}$ are real and because of the Hermitian property this leads to

$$
H_{n m}=H_{m n} .
$$

Thus, these systems are represented by real symmetric matrices and the Hermiticity is preserved under orthogonal transformations using orthogonal matrices $O$ with $O O^{T}=\mathrm{Id}$

$$
\mathbf{H}^{\prime}=\mathbf{O H O}^{T} \text {. }
$$

Consequently, these system will be related to orthogonal ensembles.

In the remaining case there is an additional spin- $1 / 2$ interaction. The commutator (A.27) does not vanish since the Hamiltonian now contains complex parts. As these systems are of no interest in this study we will just present the result. Substituting the Pauli-matrices from the spin operator with quaternions it is possible to define another operator $\hat{\tilde{C}}$ that commutes with $\hat{H}$. This property is preserved unter symplectic transformations

$$
\mathbf{H}^{\prime}=\mathbf{S H S}^{R}
$$

where $\mathbf{S}$ is a symplectic matrix with $\mathbf{S} \mathbf{S}^{R}=\operatorname{Id}$ and $\mathbf{S}^{R}$ being its dual. 


\section{A.1.3. Unfolding spectra}

Consider a Hamiltonian with a discrete spectrum

$$
E_{1} \leq E_{2} \cdots \leq E_{n} \leq \cdots
$$

where $E_{n}$ are the ordered eigenenergies. To count the number of populated levels at a certain Energy we introduce the staircase function

$$
N(E)=\sum_{i=1}^{\infty} \theta\left(E-E_{i}\right)=\sum_{E_{i} \leq E} 1=\operatorname{Tr} \theta(\mathbf{E}-\mathbf{H})
$$

The density of states $g(E)$ is its derivative

$$
g(E)=\frac{d N}{d E}=\sum_{i=1}^{\infty} \delta\left(E-E_{i}\right)=\operatorname{Tr} \delta(\mathbf{E}-\mathbf{H})
$$

Our aim is now to separate the smooth averaged part of $N(E)$ from the oscillating one

$$
N(E)=\langle N(E)\rangle+N_{\text {osc }}(E)
$$

From that definition the averaged density of states is

$$
\langle g(E)\rangle=\frac{d\langle N(E)\rangle}{d E}
$$

Our main interest lies in the fluctuations around the mean rather than in the mean itself. Now a problem arises when we want to compare spectra from different systems. How shall we separate the mean from the oscillations? One method ([33]) is to map the original sequence of eigenenergies to a sequence with a constant average density equal to unity, the so called unfoldingof the spectrum:

Let $\left\{E_{i}\right\}$ be the original sequence of energies. With

$$
\left\{x_{i}\right\}=\left\{\left\langle N\left(E_{i}\right)\right\rangle\right\}
$$

we get

$$
\langle N(x)\rangle=x
$$

With this mapping we are now in the position to compare properties of different spectra such as the level spacings $S_{n}$.

\section{A.2. Semiclassical bandprofile}

Here we rederive a semiclassical expression found by Feingold and Peres in 1986 [85, 171] that relates the power spectrum of the generalized force $\mathcal{F}$ to the quantum mechanical 
profile of the corresponding matrix $\mathbf{B}$. Consider the operator $\hat{B}$ with well-defined classical limit $\mathcal{B}$. We start with the classical correlator $C_{j}(\tau)$ where curly brackets \{\}$_{j}$ denote the classical microcanonical average corresponding to energy $E_{j}$

$$
\begin{aligned}
C_{j}(\tau)= & \{\mathcal{B}(\tau) \mathcal{B}(0)\}_{j} \\
\stackrel{\hbar \rightarrow 0}{=} & \langle j|\hat{B}(\tau) \hat{B}(0)| j\rangle \\
= & \left\langle j\left|e^{\frac{i \mathcal{H}_{0} \tau}{\hbar}} \hat{B} e^{\frac{-i \mathcal{H}_{0} \tau}{\hbar}} \hat{B}\right| j\right\rangle \\
& \sum_{k}\left\langle j\left|e^{i E_{j} \tau / \hbar} \hat{B}\right| k\right\rangle e^{-i E_{k} \tau / \hbar}\langle k|\hat{B}| j\rangle \\
= & \sum_{k} e^{i\left(E_{j}-E_{k}\right) \tau / \hbar}\left|B_{j k}\right|^{2}
\end{aligned}
$$

Now we expressed the classical correlator in term of the transition elements $B_{j k}=\langle j|\mathcal{B}| k\rangle$. In the second equality we applied the generalized Shnirelman theorem, which states that in the semiclassical limit the quantum mechanical expression is equal to the classical average. Because of the ergodicity, the microcanonical and the temporal average coincide

$$
C_{j}(\tau)=\lim _{T \rightarrow \infty} \frac{1}{T} \int_{-\frac{T}{2}}^{\frac{T}{2}} \mathrm{~d} t \mathcal{B}(\tau+t) \mathcal{B}(t)
$$

If we now replace the sum $\sum_{k}$ with the integral $\int \mathrm{d} E_{k} g\left(E_{k}\right)$, where $g(E)$ is the density of states, and identify the result with the Fourier back-transform of the the classical power spectrum, i.e., the correlator

$$
C(\tau)=\frac{1}{2 \pi} \int_{-\infty}^{\infty} \tilde{C}(\omega) e^{i \omega \tau} \mathrm{d} \omega
$$

we obtain

$$
\left\langle\left|B_{j k}\right|^{2}\right\rangle_{j}=\frac{\tilde{C}\left(\omega=\frac{E_{j}-E_{k}}{\hbar}\right)}{2 \pi \hbar g\left(E_{k}\right)} .
$$

This is the orginial result of Feingold and Peres. In order to apply this to the BHH system and obtain the scaled version of Eq. (3.14) we rewrite the above correlator (A.43) using $\mathcal{B}=-\frac{\partial \mathcal{H}}{\partial k}$ : 


$$
\begin{aligned}
C_{j}(\tau) & =\int \mathrm{d} t \frac{\partial \mathcal{H}}{\partial k}(\tau+t) \frac{\partial \mathcal{H}}{\partial k}(t) \quad ; \tilde{\mathcal{H}}=\frac{\mathcal{H}}{N \tilde{U}} \\
& =(N \tilde{U})^{2} \int \mathrm{d} t \frac{\partial \tilde{\mathcal{H}}}{\partial k}(\tau+t) \frac{\partial \tilde{\mathcal{H}}}{\partial k}(t) \quad ; \lambda=\frac{k}{\tilde{U}} \\
& =N^{2} \int \mathrm{d} t \frac{\partial \tilde{\mathcal{H}}}{\partial \lambda}(\tau+t) \frac{\partial \tilde{\mathcal{H}}}{\partial \lambda}(t) \quad ; \tilde{t}=\tilde{U} t \\
& =\frac{N^{2}}{\tilde{U}} \int \mathrm{d} \tilde{t} \frac{\partial \tilde{\mathcal{H}}}{\partial \lambda}(\tilde{\tau}+\tilde{t}) \frac{\partial \tilde{\mathcal{H}}}{\partial \lambda}(\tilde{t}) \\
& =\frac{N^{2}}{\tilde{U}} C^{\prime}(\tilde{\tau}) .
\end{aligned}
$$

In the last step, $C^{\prime}(\tilde{\tau})$ denotes the scaled correlation function. From this we obtain immediately

$$
\tilde{C}(\omega)=\frac{N^{2}}{\tilde{U}} \tilde{C}^{\prime}(\tilde{\omega})
$$




\section{B. Adiabatic Pumping and Transport in a Two-level System}

\section{B.1. Adiabatic pumping}

The theoretical description of a quantum pump is closely related to parametric Hamiltonians $\mathcal{H}(q, p, X(t))$, a concept which was introduced in Chapter 3. In order to generate a current, the system is driven by changing a control parameter $X(t)$ in time. Let us assume that we have three independent parameters $X=\left(X_{1}, X_{2}, X_{3}\right)$. In Fig. B.1 we show various other systems that can be used to implement a quantum pump: In the first case (Fig. B.1a) an atom current is initiated in a ring cavity by moving a scatterer which represents the stir. Alternatively, one can change the scatterer's position in a complex quantum graph network (Fig. B.1b) or vary delta-like potentials that represent two barriers (Fig. B.1c).

The current $I$ that results from the pumping/stirring is given by the derivative of the onsite population and can be calculated using the Heisenberg equation. Formally, it can be written as the conjugate force of a ficticious field $X_{3}$ [54]. ${ }^{1}$ This will be important in the evaluation of the elements of the geometric conductance matrix (see below). In analogy with Ohm's law we get in general

$$
I=-\sum_{j} G_{j} \dot{X}_{j}
$$

where the $G_{j}$ are elements of the generalized conductance matrix $\mathbf{G}^{j k}$.

Here we are interested in periodic (AC) driving hence the driving cycle is a closed contour in the parameter $(X)$ space. In order to apply the above formula the driving frequency $\omega$ has to be low enough such that the current depends only on the rates $\dot{X}_{j}$. Then the atomic charge $Q$ transported in one cycle is given by the contour-integral

$$
Q=\oint_{\text {cycle }} I d t=-\oint G d X .
$$

where $G=\left(G_{1}, G_{2}, G_{3}\right)$. If the current in (B.2) has a DC contribution, then we obtain a nonvanishing net charge $Q \neq 0$ during one cycle. This constitutes our definition of pumping,

\footnotetext{
${ }^{1}$ An example where the force is not ficticious but real is an Aharonov-Bohm geometry where $X_{3}=\phi$ represents a magnetic flux penetrating the hole of the $\mathrm{AB}$-ring. The time-derivative of the flux $\dot{\phi}$ is proportional to the electromotive force (EMF) $-\dot{\phi}$ and thereby induces a current $I$. To see formally that $I$ is the conjugate force to $\phi$ one considers that the energy increase $d E$ in an AB-ring due to a change in the flux is the EMF times the charge, i.e. $d E=-d X_{3} / d t \times I d t=-I d X_{3}$.
} 
a)

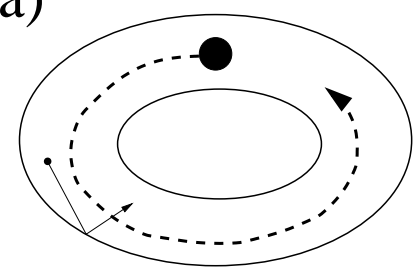

b)

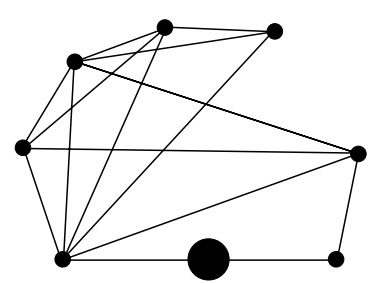

c)

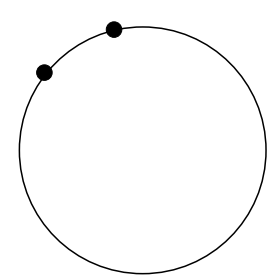

Figure B.1.: Other examples of pumping/stirring schemes. (a) a scatterer, the stir (black disk), is moved inside a ring-cavity and thereby induces a current, (b) the stir is moved in a complex quantum graph, and (c) two delta potentials (black dots) are switched on an off thereby imitating a classical peristaltic mechnism [55].

namely to get a $\mathrm{DC}$ current from an $\mathrm{AC}$ driving. From the above expression, we deduce that one needs to change at least two parameters in order to obtain $Q \neq 0 .^{2}$ In the case of the BHH trimer we change the control parameters $X_{1}$ and $X_{2}$ which correspond to the bias in coupling strength and the on-site potential at site $i=0$ respectively while $X_{3} \equiv 0$.

\section{B.1.1. The adiabatic equation and the geometric conductance}

The leading theory to describe driven systems is linear response theory (see Chapter 3) which is also used to calculate the conductance. It turns out that the generalized conductance matrix $\mathbf{G}^{j k}$ can be decomposed [54] in a symmetric and an anti-symmetric part which account for the dissipative and non-dissipative effect of the driving respectively. Here we consider a strictly adiabatic driving: Although the energy is not a constant of motion the system returns to the initial state at the end of each cycle. In this case there is no dissipation thus we consider only the anti-symmetric part of $\mathbf{G}^{j k}$ which is also referred to as the geometric conductance matrix $\mathbf{B}^{j k}$. In order to calculate the geometric conductance we use the Kubo formula approach to quantum pumping [54] which is based on the theory of adiabatic processes $[25,15,27]$. In the following we show how to obtain $\mathbf{B}^{j k}$ from the adiabatic equation following closely Ref. [53]. ${ }^{3}$

In the instantaneous basis $|\psi\rangle=\sum_{n} a_{n}|n(X(t))\rangle$ the Schrödinger equation $i \hbar \partial_{t}|\psi\rangle=$ $H(X)|\psi\rangle$ becomes

$$
\frac{d a_{n}}{d t}=-\frac{i}{\hbar} E_{n} a_{n}+\frac{i}{\hbar} \sum_{m} \sum_{j} \dot{X}_{j} \mathbf{A}_{n m}^{j} a_{m}
$$

where we defined

$$
A_{n m}^{j}=i \hbar\left\langle m \mid \frac{\partial}{\partial X_{j}} n\right\rangle
$$

\footnotetext{
${ }^{2}$ In order to get $Q \neq 0$ from one-parameter driving one has to consider nonlinear driving [55] like quantum ratchets that we don't discuss here.

${ }^{3}$ For a recent review on quantum pumping and the general derivation of the conductance matrix see Ref. [54, $55]$ and references therein.
} 


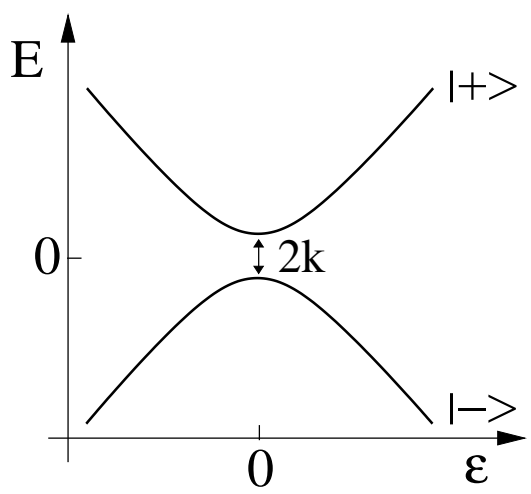

Figure B.2.: Scheme of the avoided crossing. The two-site system (B.8) is prepared in the ground state which initially $\varepsilon(t=0) \ll 0$ corresponds to the particle occupying the left site. As the potential $\varepsilon$ is raised the particle encounters an avoided crossing. Due to the adiabatic driving it stays in the ground state which for $\varepsilon \gg 0$ corresponds to the right site, i.e., the particle is transported during the avoided crossing.

Differentiation by parts of $\partial_{j}\langle m(X) \mid n(X)\rangle=0$ implies that $\mathbf{A}_{n m}^{j}$ is a Hermitian matrix. We denote its (real) diagonal elements as $\mathbf{A}_{n}^{j} \equiv \mathbf{A}_{n n}^{j}$. The latter is associated with a gaugeinvariant "two-form", defined as [54]

$$
\begin{aligned}
\mathbf{B}_{n}^{k j} & =\partial_{k} \mathbf{A}_{n}^{j}-\partial_{j} \mathbf{A}_{n}^{k} \\
& =-2 \hbar \operatorname{Im}\left\langle\partial_{k} n \mid \partial_{j} n\right\rangle \\
& =-\frac{2}{\hbar} \operatorname{Im} \sum_{m} \mathbf{A}_{n m}^{k} \mathbf{A}_{m n}^{j}
\end{aligned}
$$

Using $\partial_{X_{j}}\langle m(X)|\mathcal{H}| n(X)\rangle=0$ we find that the off-diagonal elements of $\mathbf{A}_{n m}^{j}$ can be written as

$$
\mathbf{A}_{n m}^{j}=\frac{i \hbar}{E_{m}-E_{n}}\left\langle n\left|\frac{\partial \mathcal{H}}{\partial X_{j}}\right| m\right\rangle=-\frac{i \hbar \mathcal{F}_{n m}^{j}}{E_{m}-E_{n}}
$$

and hence

$$
\mathbf{B}_{n}^{k j}=\sum_{m \neq n} \frac{2 \hbar \operatorname{Im}\left[\mathcal{F}_{n m}^{k} \mathcal{F}_{m n}^{j}\right]}{\left(E_{m}-E_{n}\right)^{2}} .
$$

In the above formulas $\mathcal{F}^{j}=-\frac{\partial}{\partial X_{j}} \mathcal{H}$ represents the generalized force corresponding the parameter $X_{j}$ (see Section 3.1.2).

During each part of the driving we change one parameter $X_{j},(j=1,2)$ at a time while the second one $X_{k}=X_{3}$ is used to measure the current. Namely, we set $X_{3}$ to be a ficticious field $\phi$ (see above). Then its conjugate force $\mathcal{F}^{3}=I$ is the current. For the BHH trimer $I$ is given by Eq. (6.6). Above the index $n$ distinguishes the eigenstates of the many-body Hamiltonian. Assuming that $n=n_{0}$ is the BEC ground state we can drop the indices $n, k$ in Eq. (B.7) and obtain Eq. (6.5). 


\section{B.2. Transport in a two-site system}

Consider a single particle system consisting of two sites coupled with a strength $k$ and onsite potentials $v_{1}=\varepsilon / 2, v_{2}=-\varepsilon / 2$. The model Hamiltonian and the current operator in the position basis are written as

$$
\mathcal{H}=\left(\begin{array}{cc}
\varepsilon / 2 & -k \\
-k & -\varepsilon / 2
\end{array}\right), \quad I=k\left(\begin{array}{cc}
0 & -i \\
i & 0
\end{array}\right) .
$$

At time $t=0$, the control parameter $\varepsilon$ is very negative and the particle is on the left site $(i=1)$. Then $\varepsilon$ is increased adiabatically and the level experiences an avoided crossing (if $k \neq 0$ ). The Landau-Zener transition probability is [140] $P_{L Z}=\exp \left[-2 \pi k_{\hbar \dot{\varepsilon}}^{k^{2}}\right]$. In the following we are working in the adiabatic limit $\left(p=1-P_{L Z} \approx 1\right)$, i.e., the particle stays always in the ground state. During the avoided crossing the particle will be transported from the left to the right site (see Fig. B.2). In the instantaneous basis $|\psi\rangle=\sum_{n} a_{n}|n(\varepsilon(t))\rangle$ the Schrödinger equation $i \partial_{t}|\psi\rangle=H(\varepsilon)|\psi\rangle$ becomes

$$
\frac{d a_{n}}{d t}=-i E_{n} a_{n}+i \sum_{m} \dot{\varepsilon} \mathbf{A}_{n m} a_{m}
$$

Since we are working in the adiabatic limit we can apply time-independent first order perturbation theory to the Hamiltonian $\mathcal{H}^{\prime \prime}=\mathcal{H}-\dot{\varepsilon} \mathcal{A}$ where $\mathcal{H}$ is given by (B.8) and the perturbation matrix is:

$$
A_{n m}=i\left\langle n \mid \frac{\partial}{\partial \varepsilon} m\right\rangle
$$

At time $t=-\infty$ the control parameter $\varepsilon$ is very negative and we compute the zero-order eigenenergies and eigenstates using standard textbook expression (see e.g. Cohen-Tannoudji Complement $\mathrm{B}_{\mathrm{IV}}$ )

$$
\begin{aligned}
E_{ \pm}^{[0]} & = \pm \frac{1}{2} \Omega \\
\left|-^{[0]}\right\rangle & =\left(\begin{array}{c}
\cos \left(\frac{\theta}{2}\right) \\
\sin \left(\frac{\theta}{2}\right)
\end{array}\right) \\
\left|{ }^{[0]}\right\rangle & =\left(\begin{array}{c}
-\sin \left(\frac{\theta}{2}\right) \\
\cos \left(\frac{\theta}{2}\right)
\end{array}\right)
\end{aligned}
$$

where

$$
\begin{aligned}
\Omega & =\sqrt{\varepsilon^{2}+(2 k)^{2}} \\
\theta & =-\arctan \left(\frac{2 k}{\varepsilon}\right)
\end{aligned}
$$

The expectation value of the current operator $I$ with respect to the zero-order eigenstates vanishes since the latter are independent of the generalized force $\mathcal{F}$ (which is proportional to $\dot{\varepsilon})$ : 


$$
\begin{aligned}
\left\langle-^{[0]}|I|-{ }^{[0]}\right\rangle & =i k\left(\begin{array}{c}
\cos \left(\frac{\theta}{2}\right) \\
\sin \left(\frac{\theta}{2}\right)
\end{array}\right)\left(\begin{array}{c}
-\sin \left(\frac{\theta}{2}\right) \\
\cos \left(\frac{\theta}{2}\right)
\end{array}\right)=0 \\
& =i k\left(\begin{array}{c}
-\sin \left(\frac{\theta}{2}\right) \\
\cos \left(\frac{\theta}{2}\right)
\end{array}\right)\left(\begin{array}{c}
-\cos \left(\frac{\theta}{2}\right) \\
-\sin \left(\frac{\theta}{2}\right)
\end{array}\right)=\left\langle+^{[0]}|I|+{ }^{[0]}\right\rangle .
\end{aligned}
$$

The first order correction to the eigenstates of this two-level system is

$$
\left| \pm^{[1]}\right\rangle=\frac{\left\langle\mp^{[0]}|A| \pm^{[0]}\right\rangle}{E_{ \pm}^{[0]}-E_{\mp}^{[0]}}\left|\mp^{[0]}\right\rangle .
$$

and with $\partial / \partial x \arctan (x)=1 /\left(1+x^{2}\right)$ we get $\partial \theta / \partial \varepsilon=2 k / \Omega^{2}$ and find

$$
A_{m n}=i\left\langle m \mid \frac{\partial}{\partial \varepsilon} n\right\rangle=\frac{k}{\Omega^{2}}\left(\begin{array}{cc}
0 & -i \\
i & 0
\end{array}\right) .
$$

Consequently, to first order, the eigenstates are

$$
\begin{aligned}
|+\rangle & =\left|+{ }^{[0]}\right\rangle-i \dot{\varepsilon} \frac{k}{\Omega^{3}}\left|-{ }^{[0]}\right\rangle \\
|-\rangle & =\left|-{ }^{[0]}\right\rangle-i \dot{\varepsilon} \frac{k}{\Omega^{3}}\left|+{ }^{[0]}\right\rangle .
\end{aligned}
$$

For these states the expectation value of the current operator becomes non-zero

$$
\begin{aligned}
\langle \pm|I| \pm\rangle & =\left[\left\langle \pm^{[0]}\left|+i \dot{\varepsilon} \frac{k}{\Omega^{3}}\left\langle\mp^{[0]}\right|\right] k\left(\begin{array}{cc}
0 & -i \\
i & 0
\end{array}\right)\left[\left| \pm^{[0]}\right\rangle-i \dot{\varepsilon} \frac{k}{\Omega^{3}}\left|\mp{ }^{[0]}\right\rangle\right]\right.\right. \\
& =\mp 2 \dot{\varepsilon} \frac{k^{2}}{\Omega^{3}} .
\end{aligned}
$$

Using the notation $\langle I\rangle=-G \dot{\varepsilon}$, where $G$ is the geometric conductance we get for a preparation in the ground state

$$
G=-\frac{2 k^{2}}{\left[\varepsilon^{2}+(2 k)^{2}\right]^{3 / 2}} .
$$

The charge is obtained by integrating over the time, which becomes an integral over the $\varepsilon(t)$ curve in parameter space. For a ground state preparation we get

$$
\langle Q\rangle=\int\langle I\rangle d t=-\int G d \varepsilon=\left.\frac{\varepsilon}{2 \sqrt{\varepsilon^{2}+(2 k)^{2}}}\right|_{-\infty} ^{\infty}=1 .
$$




\section{Bibliography}

[1] Albiez, M., Gati, R., Folling, J., Hunsmann, S., Cristiani, M., and Oberthaler, M. K. Direct Observation of Tunneling and Nonlinear Self-Trapping in a Single Bosonic Josephson Junction. Phys. Rev. Lett. 95, 010402 (2005).

[2] Altshuler, B. L. and Glazman, L. I. Pumping Electrons. Science 283, 1864 (1999).

[3] Amico, L., Osterloh, A., and Cataliotti, F. Quantum Many Particle Systems in RingShaped Optical Lattices. Phys. Rev. Lett. 95, 063201 (2005).

[4] Andersen, J. D. and Kenkre, V. M. Exact Solutions for the Quantum Nonlinear Trimer. phys. stat. sol. 177, 397 (1993).

[5] Andersen, M. F., Grünzweig, T., Kaplan, A., and Davidson, N. Revivals of coherence in chaotic atom-optics billiards. Phys. Rev. A 69, 063413 (2004).

[6] Andersen, M. F., Kaplan, A., Grünzweig, T., and Davidson, N. Decay of Quantum Correlations in Atom Optics Billiards with Chaotic and Mixed Dynamics. Phys. Rev. Lett. 97, 104102 (2006).

[7] Anderson, B. P. and Kasevich, M. A. Macroscopic Quantum Interference from Atomic Tunnel Arrays. Science 282, 1686 (1998).

[8] Anderson, M. H., Ensher, J. R., Matthews, M. R., Wieman, C. E., and Cornell, E. A. Observation of Bose-Einstein Condensation in a Dilute Atomic Vapor. Science 269, 5221 (1995).

[9] Anderson, P. W. Absence of Diffusion in Certain Random Lattices. Phys. Rev. 109, 1492 (1957).

[10] Andersson, E., Calarco, T., Folman, R., Andersson, M., Hessmo, B., and Schmiedmayer, J. Multimode Interferometer for Guided Matter Waves. Phys. Rev. Lett. 88, 100401 (2002).

[11] Andrews, M. R., Townsend, C. G., Miesner, H.-J., Durfee, D. S., Kurn, D. M., and Ketterle, W. Observation of Interference Between Two Bose Condensates. Science 275, 637 (1997).

[12] Anglin, J. R. and Ketterle, W. Bose-Einstein condensation of atomic gases. Nature 416, 211 (2002). 
[13] Aubry, S., Flach, S., Kladko, K., and Olbrich, E. Manifestation of Classical Bifurcation in the Spectrum of the Integrable Quantum Dimer. Phys. Rev. Lett. 76, 1607 (1996).

[14] Austin, E. J. and Wilkinson, M. Distribution of Matrix Elements of a Classically Chaotic System. Europhys. Lett. 20, 589 (1992).

[15] Avron, J. E., Raveh, A., and Zur, B. Adiabatic quantum transport in multiply connected systems. Rev. Mod. Phys. 60, 873 (1988).

[16] Balian, R. From Microphysics to Macrophysics. Springer, New York (1991).

[17] Barvik, I., Esser, B., and Schanz, H. Nonlinearity and Trapping in Excitation Transfer - Dimers and Trimers. Phys. Rev. B 52, 9377 (1995).

[18] Batrouni, G. G., Assaad, F. F., Scalettar, R. T., and Denteneer, P. J. H. Dynamic response of trapped ultracold bosons on optical lattices. Phys. Rev. A 72, 031601(R) (2005).

[19] Beenakker, C. W. J. Random-matrix theory of quantum transport. Rev. Mod. Phys. 69, 731 (1997).

[20] Benenti, G. and Casati, G. Quantum-classical correspondence in perturbed chaotic systems. Phys. Rev. E 65, 066205 (2002).

[21] Bernstein, L., Eilbeck, J. C., and Scott, A. C. The quantum theory of local modes in a coupled system of nonlinear oscillators. Nonlinearity 3, 293 (1990).

[22] Bernstein, L. J. The three-waveguide nonlinear directional coupler: the center waveguide excitation. Opt. Commun. 94, 406 (1992).

[23] Bernstein, L. J., DeLong, K. W., and Finlayson, N. Self-trapping transitions in a discrete NLS model with localized initial conditions. Phys. Lett. A 181, 135 (1993).

[24] Berry, M. V. Regular and irregular semiclassical wavefunctions. J. Phys. A 10, 2083 (1977).

[25] Berry, M. V. Quantal Phase Factors Accompanying Adiabatic Changes. Proc. of the Royal Society of London. Series A 392, 45 (1984).

[26] Berry, M. V. Chaos and Quantum Systems. Elsevier (1991).

[27] Berry, M. V. and Robbins, J. M. Classical Geometric Forces of Reaction: An Exactly Solvable Model. Proc. of the Royal Society of London. Series A 442, 641 (1993).

[28] Berry, M. V. and Robnik, M. Semiclassical level spacings when regular and chaotic orbits coexist. J. Phys. A 17, 2413 (1984). 
[29] Bialynicki-Birula, I. and Bialynicka-Birula, Z. Rotational Frequency Shift. Phys. Rev. Lett. 78, 2539 (1997).

[30] Bloch, I., Hänsch, T. W., and Esslinger, T. Atom Laser with a cw Output Coupler. Phys. Rev. Lett. 82, 3008 (1998).

[31] Bodyfelt, J. D., Hiller, M., and Kottos, T. Engineering fidelity echoes in BoseHubbard Hamiltonians. Europhys. Lett. 78, 50003 (2007).

[32] Bogoliubov, N. N. J. Phys. USSR 11, 23 (1947).

[33] Bohigas, O. Session LII. North Holland (1989).

[34] Bohigas, O., Giannoni, M. J., and Schmit, C. Characterization of chaotic spectra and universality of level fluctuation laws. Phys. Rev. Lett. 52, 1 (1984).

[35] Bose, S. N. Planck's law and light quantum hypothesis. Z. Phys. 26, 178 (1924).

[36] Brody, T. A. Statistical measure for repulsion of energy-levels. Lett. Nouvo Cimento 12, 482 (1973).

[37] Brody, T. A., Flores, J., Fench, J. B., Mello, P. A., Pandey, A., and Wong, S. S. M. Random-matrix physics: spectrum and strength fluctuations. Rev. Mod. Phys. 53, 385 (1981).

[38] Brouwer, P. W. Scattering approach to parametric pumping. Phys. Rev. B 58, R10135 (1998).

[39] Bruder, C., Fazio, R., and Schön, G. The Bose-Hubbard model: from Josephson junction arrays to optical lattices. Ann. d. Physik 14, 566 (2005).

[40] Bulgac, A., Dang, G. D., and Kusnezov, D. Random matrix approach to quantum dissipation. Phys. Rev. E 45, 3468 (1996).

[41] Buonsante, P., Franzosi, R., and Penna, V. Dynamical Instability in a Trimeric Chain of Interacting Bose-Einstein Condensates. Phys. Rev. Lett. 90, 050404 (2003).

[42] Büttiker, M., Thomas, H., and Prêtre, A. Current partition in multiprobe conductors in the presence of slowly oscillating external potentials. Z. Phys. B Condens. Mat. 94, 133 (1994).

[43] Carusotto, I. and La Rocca, G. C. Modulated Optical Lattice as an Atomic FabryPerot Interferometer. Phys. Rev. Lett. 84, 399 (2000).

[44] Casati, G., Chirikov, B. V., and Guarneri, I. Energy-Level Statistics of Integrable Quantum Systems. Phys. Rev. Lett. 54, 1350 (1985). 
[45] Casati, G., Chirikov, B. V., Guarneri, I., and Izarailev, F. M. Band-random-matrix model for quantum localization in conservative systems. Phys. Rev. E 48, R1613 (1993).

[46] Casetti, L., Pettini, M., and Cohen, E. G. D. Geometric approach to Hamiltonian dynamics and statistical physics. Phys. Rep. 337, 237 (2000).

[47] Cataliotti, F. S., Burger, S., Fort, C., Maddaloni, P., Minardi, F., Trombettoni, A., Smerzi, A., and Inguscio, M. Josephson Junction Arrays with Bose-Einstein Condensates. Science 293, 843 (2001).

[48] Cataliotti, F. S., Fallani, L., Ferlaino, F., Fort, C., Maddaloni, P., and Inguscio, M. Superfluid current disruption in a chain of weakly coupled Bose-Einstein condensates. New J. Phys. 5, 71 (2003).

[49] Chefles, A. Nearest-Neighbour level spacings for the non-periodic discrete Schrödinger equation. J. Phys. A. 29, 4515 (1996).

[50] Chuu, C.-S., Schreck, F., Meyrath, T. P., Hanssen, J. L., Price, G. N., and Raizen, M. G. Direct Observation of Sub-Poissonian Number Statistics in a Degenerate Bose Gas. Phys. Rev. Lett. 95, 260403 (2005).

[51] Clément, D., Varón, A. F., Hugbart, M., Retter, J. A., Bouyer, P., Sanchez-Palencia, L., Gangardt, D. M., Shlyapnikov, G. V., and Aspect, A. Suppression of Transport of an Interacting Elongated Bose-Einstein Condensate in a Random Potential. Phys. Rev. Lett. 95, 170409 (2005).

[52] Cohen, D. Chaos and Energy Spreading for Time-Dependent Hamiltonians, and the Various Regimes in the Theory of Quantum Dissipation. Ann. Phys. 283, 175 (2000).

[53] Cohen, D. Lecture notes: The adiabatic formalism, linear response theory, and the Born-Oppenheimer picture (2003).

[54] Cohen, D. Quantum pumping in closed systems, adiabatic transport, and the Kubo formula. Phys. Rev. B 68, 155303 (2003).

[55] Cohen, D. Quantum pumping and dissipation in closed systems. Physica E 29, 308 (2005).

[56] Cohen, D. and Heller, E. J. Unification of Perturbation Theory, RMT and Semiclassical Considerations in the Study of Parametrically Dependent Eigenstates. Phys. Rev. Lett. 84, 2841 (2000).

[57] Cohen, D., Izrailev, F. M., and Kottos, T. Wave Packet Dynamics in Energy Space, Random Matrix Theory, and the Quantum-Classical Correspondence. Phys. Rev. Lett. 84, 2052 (2000). 
[58] Cohen, D. and Kottos, T. Quantum-Mechanical Nonperturbative Response of Driven Chaotic Mesoscopic Systems. Phys. Rev. Lett. 85, 4839 (2000).

[59] Cohen, D. and Kottos, T. Parametric dependent Hamiltonians, wave functions, random matrix theory, and quantal-classical correspondence. Phys. Rev. E 63, 036203 (2001).

[60] Cohen, D. and Kottos, T. Non-perturbative response: chaos versus disorder. J. Phys. A 36, 10151 (2003).

[61] Cohen-Tannoudji, C. Atomic Motion in Laser Light. North Holland (1992).

[62] Cohen-Tannoudji, C. N. Nobel Lecture: Manipulating atoms with photons. Rev. Mod. Phys. 70, 707 (1998).

[63] Corney, J. F. and Milburn, G. J. Homodyne measurements on a Bose-Einstein condensate. Phys. Rev. A 58, 2399 (1998).

[64] Cruzeiro-Hansson, L., Feddersen, H., Flesch, R., Christiansen, P. L., Salerno, M., and Scott, A. C. Classical and quantum analysis of chaos in the discrete self-trapping equation. Phys. Rev. B 42, 522 (1990).

[65] Cucchietti, F. M., Dalvit, D. A. R., Paz, J. P., and Zurek, W. H. Decoherence and the Loschmidt Echo. Phys. Rev. Lett. 91, 210403 (2003).

[66] Cucchietti, F. M., Lewenkopf, C. H., Mucciolo, E. R., Pastawski, H. M., and Vallejos, R. O. Measuring the Lyapunov exponent using quantum mechanics. Phys. Rev. E 65, 046209 (2002).

[67] Dalfovo, F., Giorgini, S., Pitaevskii, L. P., and Stringari, S. Theory of Bose-Einstein condensation in trapped gases. Rev. Mod. Phys. 71, 463 (1999).

[68] Damski, B., Zakrzewski, J., Santos, L., Zoller, P., and Lewenstein, M. Atomic Bose and Anderson Glasses in Optical Lattices. Phys. Rev. Lett. 91, 080403 (2003).

[69] Davis, K. B., Mewes, M.-O., Andrews, M. R., van Druten, N. J., Durfee, D. S., Kurn, D. M., and Ketterle, W. Bose-Einstein Condensation in a Gas of Sodium Atoms. Phys. Rev. Lett 75, 3973 (1995).

[70] de Filippo, S., Fusco Girard, M., and Salerno, M. Lyapunov Exponents for the $n=3$ discrete self-trapping equation. Physica D 26, 411 (1987).

[71] de Filippo, S., Fusco Girard, M., and Salerno, M. Avoided crossing and nearestneighbour level spacings for the quantum DST equation. Nonlinearity 2, 477 (1989).

[72] Dittes, F. The decay of quantum systems with a small number of open channels. Phys. Rep. 339, 216 (2000). 
[73] Dounas-Frazer, D. R. and Carr, L. D. Tunneling resonances and entanglement dynamics of cold bosons in the double well. arXiv quant-ph/0610166 (2006).

[74] Dudarev, A. M., Raizen, M. G., and Niu, Q. Quantum Many-Body Culling: Production of a Definite Number of Ground-State Atoms in a Bose-Einstein Condensate. Phys. Rev. Lett. 98, 063001 (2007).

[75] Eilbeck, J. C. and Johansson, M. The discrete nonliner Schrödinger equation - 20 years on. Proc. of the 3rd Conf. Localization and Energy Transfer in Nonlinear Systems (World Scientific, New Jersey, 2003).

[76] Eilbeck, J. C., Lomdahl, P. S., and Scott, A. C. The discrete self-trapping equation. Physica D 16, 318 (1985).

[77] Eilbeck, J. C., Tsironis, G. P., and Turitsyn, S. K. Stationary states in a doubly nonlinear trimer model of optical couplers. Phys. Scr. 52, 386 (1995).

[78] Einstein, A. Quantentheorie des einatomigen idealen Gases. Sitzungsber. Preuss. Akad. Wiss., Phys. Math. Kl. page 261 (1924).

[79] Einstein, A. Quantentheorie des einatomigen idealen Gases - Zweite Abhandlung. Sitzungsber. Preuss. Akad. Wiss., Phys. Math. Kl. page 3 (1925).

[80] Ellegaard, C., Guhr, T., Lindemann, K., Nygard, J., and Oxborrow, M. Symmetry Breaking and Spectral Statistics of Acoustic Resonances in Quartz Blocks. Phys. Rev. Lett 77, 4918 (1996).

[81] Fazio, R. and van der Zant, H. Quantum phase transitions and vortex dynamics in superconducting networks. Phys. Rep. 355, 235 (2001).

[82] Feddersen, H. Localization of vibrational energy in globular protein. Phys. Lett. A 154, 391 (1991).

[83] Feingold, M., Gioletta, A., Izrailev, F. M., and Molinari, L. Two-Parameter Scaling in the Wigner Ensemble. Phys. Rev. Let 70, 2936 (1993).

[84] Feingold, M., Leitner, D. M., and Wilkinson, M. Spectral Statistics in Semiclassical Random-Matrix-Ensembles. Phys. Rev. Lett. 66, 986 (1991).

[85] Feingold, M. and Peres, A. Distribution of matrix elements of chaotic systems. Phys. Rev. A 34, 591 (1986).

[86] Fiete, G. A. and Heller, E. J. Semiclassical theory of coherence and decoherence. Phys. Rev. A 68, 022112 (2003).

[87] Finlayson, N. and Stegeman, G. I. Spatial switching, instabilities, and chaos in a three-waveguide nonlinear directional coupler. App. Phys. Lett. 56, 2276 (1990). 
[88] Fisher, M. P. A., Weichmann, P. B., Grinstein, G., and Fisher, D. S. Boson localization and the superfluid-insulator transition. Phys. Rev. B 40, 546 (1989).

[89] Flach, S. and Fleurov, V. Tunnelling in the nonintegrable trimer - a step towards quantum breathers. J. Phys.: Condens. Mat. 9, 7039 (1997).

[90] Folman, R., Krüger, P., Cassettari, D., Hessmo, B., Maier, T., and Schmiedmayer, J. Controlling Cold Atoms using Nanofabricated Surfaces: Atom Chips. Phys. Rev. Lett. 84, 4749 (2000).

[91] Fort, C., Fallani, L., Guarrera, V., Lye, J. E., Wiersma, D. S., and Inguscio, M. Effect of Optical Disorder and Single Defects on the Expansion of a Bose-Einstein Condensate in a One-Dimensional Waveguide. Phys. Rev. Lett. 95, 170410 (2005).

[92] Franzosi, R. and Penna, V. Self-trapping mechanisms in the dynamics of three coupled Bose-Einstein condensates. Phys. Rev. A 65, 013601 (2002).

[93] Franzosi, R. and Penna, V. Chaotic behavior, collective modes, and self-trapping in the dynamics of three coupled Bose-Einstein condensates. Phys. Rev. E 67, 046227 (2003).

[94] Franzosi, R., Penna, V., and Zecchina, R. Quantum dynamics of coupled bosonic wells within the Bose-Hubbard picture. Int. J. Mod. Phys. B 14, 943 (2000).

[95] Fritz, J., Baller, M. K., Lang, H. P., Rothuizen, H., Vettiger, P., Meyer, E., Günterodt, H.-J., Gerber, C., and Gimzewski, J. K. Translating Biomolecular Recognition into Nanomechanics. Science 288, 316 (2000).

[96] Fyodorov, Y. V., Chubykalo, O. A., Izrailev, F. M., and Casati, G. Wigner Random Banded Matrices with Sparse Structure: Local Spectral Density of States. Phys. Rev. Lett. 76, 1603 (1996).

[97] Fyodorov, Y. V., Kottos, T., and Stöckmann, H.-J. Trends in Quantum Chaotic Scattering. J. Phys. A 38, 10761 (2005).

[98] Garcia-Ripoll, J. J., Zoller, P., and Cirac, J. I. Quantum information processing with cold atoms and trapped ions. J. Phys. B 38, S567 (2005).

[99] Gorin, T., Prosen, T., and Seligman, T. H. A random matrix formulation of fidelity decay. New J. Phys. 6, 20 (2004).

[100] Gorin, T., Prosen, T., Seligman, T. H., and Strunz, W. T. Connection between decoherence and fidelity decay in echo dynamics. Phys. Rev. A 70, 042105 (2004).

[101] Gorin, T., Prosen, T., Seligman, T. H., and Znidaric, M. Dynamics of Loschmidt echoes and fidelity decay. Phys. Rep. 435, 33 (2006). 
[102] Greiner, M., Mandel, O., Esslinger, T., Hänsch, T. W., and Bloch, I. Quantum phase transition from a superfluid to a Mott insulator in a gas of ultracold atoms. Nature 415, 39 (2002).

[103] Gross, E. P. Structure of a Quantized Vortex in Boson Systems. Nouvo Cimento 20, 454 (1961).

[104] Gross, E. P. Hydrodynamics of a Superfluid Condensate. J. Math. Phys. 4, 195 (1963).

[105] Gustavson, T. L., Chikkatur, A. P., Leanhardt, A. E., Görlitz, A., Gupta, S., Pritchard, D. E., and Ketterle, W. Transport of Bose-Einstein Condensates with Optical Tweezers. Phys. Rev. Lett. 88, 020401 (2002).

[106] Haake, F. Quantum Signatures of Chaos. Springer-Verlag, Berlin, Heidelberg, New York, second edition (2000).

[107] Hagley, E. W., Deng, L., Kozuma, M., Wen, J., Helmerson, K., Rolston, S. L., and Phillips, W. D. A Well-Collimated Quasi-Continous Atom Laser. Science 283, 1706 (1999).

[108] Hänsel, W., Hommelhoff, P., Hänsch, T. W., and Reichel, J. Bose-Einstein condensation on a microelectronic chip. Nature 413, 498 (2001).

[109] Hänsel, W., Reichel, J., Hommelhoff, P., and Hänsch, T. W. Magnetic Conveyor Belt for Transporting and Merging Trapped Atom Clouds. Phys. Rev. Lett. 86, 608 (2001).

[110] Hennig, D., Gabriel, H., Jorgensen, M. F., Christiansen, P. L., and Clausen, C. B. Homoclinic chaos in the discrete self-trapping trimer. Phys. Rev. E 51, 2870 (1995).

[111] Hennig, D. and Tsironis, G. P. Wave transmission in nonlinear lattices. Phys. Rep. 307, 333 (1998).

[112] Hiller, M., Cohen, D., Geisel, T., and Kottos, T. Wavepacket dynamics, quantum reversibility, and random matrix theory. Ann. Phys. 321, 1025 (2006).

[113] Hiller, M., Kottos, T., and Cohen, D. Control of atomic currents using a quantum stirring device. Europhys. Lett. 82, 40006 (2008).

[114] Hiller, M., Kottos, T., and Cohen, D. Controlled quantum stirring of Bose-Einstein condensates. Phys. Rev. A 78, 013602 (2008).

[115] Hiller, M., Kottos, T., and Geisel, T. Complexity in parametric Bose-Hubbard Hamiltonians and structural analysis of eigenstates. Phys. Rev. A 73, 061604(R) (2006). 
[116] Hiller, M., Kottos, T., and Geisel, T. Wavepacket dynamics in energy space of a chaotic trimeric Bose-Hubbard system. submitted to Phys. Rev. A (2008).

[117] Hiller, M., Kottos, T., and Ossipov, A. Bifurcations in resonance widths of an open Bose-Hubbard dimer. Phys. Rev. A 73, 063625 (2006).

[118] Imry, Y. Introduction to Mesoscopic Physics. Oxford University Press, New York, Oxford (1997).

[119] Inouye, S., Andrews, M. R., Stenger, J., Miesner, H.-J., Stamper-Kurn, D. M., and Ketterle, W. Observation of Feshbach resonances in a Bose-Einstein condensate. Nature 392, 151 (1998).

[120] Iucci, A., Cazalilla, M. A., Ho, A. F., and Giamarchi, T. Energy absorption of a Bose gas in a periodically modulated optical lattice. Phys. Rev. A 73, 041608(R) (2006).

[121] Izrailev, F., Kottos, T., Politi, A., and Tsironis, G. P. Evolution of wave packets in quasi-one-dimensional and one-dimensional random media: Diffusion versus localization. Phys. Rev. E 55, 4951 (1997).

[122] Jääskeläinen, M. and Meystre, P. Coherence dynamics of two-mode condensates in asymmetric potentials. Phys. Rev. A 73, 013602 (2006).

[123] Jacquod, P., Adagideli, I., and Beenakker, C. W. J. Anomalous power law of quantum reversibility for classically regular dynamics. Europhys. Lett. 61, 729 (2003).

[124] Jacquod, P., Silvestrov, P. G., and Beenakker, C. W. J. Golden rule decay versus Lyapunov decay of the quantum Loschmidt echo. Phys. Rev. E 64, 055203(R) (2001).

[125] Jaksch, D., Bruder, C., Cirac, J. I., Gardiner, C. W., and Zoller, P. Cold Bosonic Atoms in Optical Lattices. Phys. Rev. Lett. 81, 3108 (1998).

[126] Jalabert, R. A. and Pastawski, H. Environment-independent decoherence rate in classically chaotic systems. Phys. Rev. Lett. 86, 2490 (2001).

[127] Superconductor-Insulator Transition of Underdoped Cuprates, MaNEP, National Centre of Competence in Research, Switzerland. http://www.manep.ch/en/ research-teams/beck.html (2007).

[128] Kalosakas, G. and Bishop, A. R. Small-tunneling-amplitude boson-Hubbard dimer: Stationary states. Phys. Rev. A 65, 043616 (2002).

[129] Kalosakas, G., Bishop, A. R., and Kenkre, V. M. Small-tunneling-amplitude bosonHubbard dimer: II. Dynamics. Phys. Rev. A 68, 023602 (2003). 
[130] Kasper, A., Schneider, S., vom Hagen, C., Bartenstein, M., Engeser, B., Schumm, T., Bar-Joseph, I., Folman, R., Feenstra, L., and Schmiedmayer, J. A Bose-Einstein condensate in a microtrap. J. Opt. B 5, S143 (2003).

[131] Kenkre, V. M. and Campbell, D. K. Self-trapping on a dimer - Time-dependent Solutions of a Discrete Nonlinear Schrödinger Equation. Phys. Rev. B 34, R4959 (1986).

[132] Kenkre, V. M. and Tsironis, G. P. Nonlinear effects in quasielastic neutron scattering: Exact line-shape calculation for a dimer. Phys. Rev. B 35, 1473 (1987).

[133] Ketterle, W. Nobel lecture: When atoms behave as waves: Bose-Einstein condensation and the atom laser. Rev. Mod. Phys. 74, 1131 (2002).

[134] Khomeriki, R. Nonlinear Band Gap Transmission in Optical Waveguide Arrays. Phys. Rev. Lett. 92, 063905 (2004).

[135] Kohler, S. and Sols, F. Oscillatory Decay of a Two-Component Bose-Einstein Condensate. Phys. Rev. Lett. 89, 060403 (2002).

[136] Kolovsky, A. R. and Buchleitner, A. Quantum chaos in the Bose-Hubbard model. Europhys. Lett. 68, 632 (2004).

[137] Kottos, T. and Cohen, D. Failure of random matrix theory to correctly describe quantum dynamics. Phys. Rev. E 64, 0652021 (2001).

[138] Kottos, T. and Cohen, D. Quantum irreversibility of energy spreading. Europhys. Lett. 61, 431 (2003).

[139] Kottos, T. and Weiss, M. Current Relaxation in Nonlinear Random Media. Phys. Rev. Lett. 93, 190604 (2004).

[140] Landau, L. D. and Lifshitz, E. M. Quantum Mechanics. Oxford: Pergamon (1958).

[141] Leggett, A. J. Bose-Einstein condensation in the alkali gases: Some fundamental concepts. Rev. Mod. Phys. 73, 307 (2001).

[142] Liu, J., Wu, B., and Niu, Q. Nonlinear Evolution of Quantum States in the Adiabatic Regime. Phys. Rev. Lett. 90, 170404 (2003).

[143] Lundh, E. Dipole and monopole modes in the Bose-Hubbard model in a trap. Phys. Rev. A 70, 033610 (2004).

[144] Lye, J. E., Fallani, L., Modugno, M., Wiersma, D. S., and Fort, M., Inguscio. BoseEinstein Condensate in a Random Potential. Phys. Rev. Lett. 95, 070401 (2005). 
[145] Madison, K. W., Chevy, F., Wohlleben, W., and Dalibard, J. Vortex Formation in a Stirred Bose-Einstein Condensate. Phys. Rev. Lett. 84, 806 (2000).

[146] Mahaux, C. and Weidenmüller, H. A. Shell Model Approach in Nuclear Reactions. North-Holland (1969).

[147] Mahmud, K. W., Perry, H., and Reinhardt, W. P. Phase engineering of controlled entangled number states in a single component Bose-Einstein condensate in a double well. J. Phys. B 36, L265 (2003).

[148] Mandel, L. and Wolf, E. Optical Coherence and Quantum Optics. Cambridge University Press (1995).

[149] Mewes, M.-O., Andrews, M. R., Kurn, D. M., Durfee, D. S., Townsend, C. G., and Ketterle, W. Output Coupler for Bose-Einstein Condensed Atoms. Phys. Rev. Lett. 78, 582 (1997).

[150] Micheli, A., Daley, A. J., Jaksch, D., and Zoller, P. Single Atom Transistor in a 1D Optical Lattice. Phys. Rev. Lett. 83, 140408 (2004).

[151] Milburn, G. J., Corney, J., Wright, E. M., and Walls, D. F. Quantum dynamics of an atomic Bose-Einstein condensate in a double-well potential. Phys. Rev. A 55, 4318 (1997).

[152] Moiseyev, N., Carr, L. D., Malomed, B. A., and Band, Y. B. Transition from resonances to bound states in nonlinear systems: applications to Bose-Einstein condensates. J. Phys. B 37, L193 (2004).

[153] Morsch, O. and Oberthaler, M. Dynamics of Bose-Einstein condensates in optical lattices. Rev. Mod. Phys. 78, 179 (2006).

[154] Nayfeh, M. H., Taylor, K. T., and Clark, C. W. Atomic Spectra and Collsisions in External Fields, volume 2. Plenum, New York (1989).

[155] Nemoto, K., Holmes, C. A., Milburn, G. J., and Munro, W. J. Quantum dynamics of three coupled atomic Bose-Einstein condensates. Phys. Rev. A 63, 013604 (2001).

[156] Ng, G. S. Signatures of Phase Transition in Wave Dynamics of Complex Systems. Master's thesis, Wesleyan University (2008).

[157] Nielsen, M. A. and Chuang, I. L. Quantum computation and quantum information. Cambridge UP (2000).

[158] Niu, Q. and Thouless, D. J. Quantised adiabatic charge transport in the presence of substrate disorder and many-body interaction. J. Phys. A 17, 2453 (1984). 
[159] Orzel, C., Tuchman, A. K., Fenselau, M. L., Yasuda, M., and Kasevich, M. A. Squeezed States in a Bose-Einstein Condensate. Science 291, 2386 (2001).

[160] Ott, H., Fortagh, J., Schlotterbeck, J., Grossmann, G., and Zimmermann, C. BoseEinstein condensation in a surface microtrap. Phys. Rev. Lett. 87, 230401 (2001).

[161] Pastawski, H. M., Levstein, P. R., Usaj, G., Raya, J., and Hirschinger, J. A nuclear magnetic resonance answer to the Boltzmann-Loschmidt controversy? Physica A 283, 166 (1993).

[162] Paul, R., Richter, K., and Schlagheck, P. Nonlinear resonant transport of BoseEinstein condensates. Phys. Rev. Lett. 94, 020404 (2005).

[163] Peres, A. Stability of quantum motion in chaotic and regular systems. Phys. Rev. A 30, 1610 (1984).

[164] Peres, A. Quantum Theory: Concepts and Methods. Kluwer Academic Press (1995).

[165] Petitjean, C., Bevilaqua, D. V., Heller, E. J., and Jacquod, P. Displacement Echoes: Classical Decay and Quantum Freeze. Phys. Rev. Lett. 98, 164101 (2007).

[166] Pinto, R. A. and Flach, S. Quantum dynamics of localized excitations in a symmetric trimer molecule. Phys. Rev. A 73, 022717 (2006).

[167] Pitaevskii, L. P. Vortex Lines in an Imperfect Bose Gas. Sov. Phys. JETP 13, 451 (1961).

[168] Pitaevskii, L. P. and Stringari, S. Bose-Einstein Condensation. Oxford University Press, New York, Oxford (1997).

[169] Prosen, T. Statistical Properties of Matrix Elements in a Hamilton System between Integrability and Chaos. Ann. Phys. 235, 115 (1994).

[170] Prosen, T. General relation between quantum ergodicity and fidelity of quantum dynamics. Phys. Rev. E 65, 036208 (2002).

[171] Prosen, T. and Robnik, M. Distribution and fluctuation properties of transition probabilities in a system between integrability and chaos. J. Phys. A 26, L319 (1993).

[172] Prosen, T., Seligman, T. H., and Znidaric, M. Theory of quantum Loschmidt echoes. Prog. theo. Phys. Supp. 150, 200 (2003).

[173] Prosen, T. and Znidaric, M. Stability of quantum motion and correlation decay. $J$. Phys. A 35, 1455 (2002).

[174] Raghavan, S., Bishop, A. R., and Kenkre, V. M. Quantum versus semiclassical description of self-trapping: Anharmonic effects. Phys. Rev. B 59, 9929 (1999). 
[175] Raman, C., Köhl, M., Onofrio, R., Durfee, D. S., Kuklewicz, C. E., Hadzibabic, Z., and Ketterle, W. Evidence for a Critical Velocity in a Bose-Einstein Condensed Gas. Phys. Rev. Lett. 83, 2502 (1999).

[176] Reichel, J. Microchip traps and Bose-Einstein condensation. App. Phys. B: Lasers Opt. 74, 469 (2002).

[177] Rey, A. M. Ultracold bosonic atoms in optical lattices. Ph.D. thesis, University of Maryland, USA (2004).

[178] Rosenberg, G. and Cohen, D. Quantum stirring of particles in closed devices. J. Phys. A 39, 2287 (2006).

[179] Rossini, D., Calarco, T., Giovannetti, V., Montanegro, S., and Fazio, R. Decoherence induced by interacting quantum spin baths. arXiv quant-ph/0611242 (2006).

[180] Rotter, I. A Continuum Shell-Model for the Open Quantum-Mechanical Nuclear System. Rep. Prog. Phys. 54, 635 (1991).

[181] Sachdev, S. Quantum Phase Transitions. University Press, Cambridge (1999).

[182] Salerno, M. and Scott, A. C. Quantum theories for two discrete nonlinear Schrödinger equations. Nonlinearity 4, 853 (1991).

[183] Sato, M., Hubbard, B. E., Sievers, A. J., Ilic, B., Czaplewski, D. A., and Craighead, H. G. Observation of Locked Intrinsic Localized Vibrational Modes in a Micromechanical Oscillator Array. Phys. Rev. Lett. 90, 044102 (2003).

[184] Schmiedmayer, J., Folman, R., and Calarco, T. Quantum information processing with neutral atoms on an atom chip. J. Mod. Opt 49, 1375 (2002).

[185] Schollwöck, U. The density-matrix renormalization group. Rev. Mod. Phys. 77, 259 (2005).

[186] Schulte, T., Drenkelforth, S., Kruse, J., Ertmer, W., Arlt, J., Sacha, K., Zakrzewski, J., and Lewenstein, M. Routes Towards Anderson-Like Localization of BoseEinstein Condensates in Disordered Optical Lattices. Phys. Rev. Lett. 95, 170411 (2005).

[187] Schumm, T., Hofferberth, S., Andersson, L. M., Wildermuth, S., Groth, S., BarJoseph, I., Schmiedmayer, J., and Krüger, P. Matter-wave interferometry in a double well on an atom chip. Nat. Phys. 1, 57 (2005).

[188] Scott, A. C., Lomdahl, P. S., and Eilbeck, J. C. Between the local-mode and normalmode limits. Chem. Phys. Lett. 113, 29 (1985). 
[189] Seaman, B. T., Krämer, M., Anderson, D. Z., and Holland, M. J. Atomtronics: Ultracold-atom analogs of electronic devices. Phys. Rev. A 75, 023615 (2006).

[190] Sela, I. and Cohen, D. Operating a quantum pump in a closed circuit. J. Phys. A 39, 3575 (2006).

[191] Shin, Y., Saba, M., Pasquini, T. A., Ketterle, W., Pritchard, D. E., and Leanhardt, A. E. Atom Interferometry with Bose-Einstein Condensates in a Double-Well Potential. Phys. Rev. Lett. 92, 050405 (2004).

[192] So, P., Anlage, S. M., Ott, E., and Oerter, R. N. Wave Chaos Experiments with and without Time Reversal Symmetry: GUE and GOE Statistics. Phys. Rev. Lett. 74, 2662 (1995).

[193] Sokolov, V. V. and Zelevinsky, V. G. Collective dynamics of unstable quantum states. Ann. Phys. 216, 323 (1992).

[194] Steel, M. J. and Walls, D. F. Influence of pumping on the relative phase of twin-trap Bose-Einstein condensates. Phys. Rev. A 56, 3832 (1997).

[195] Stern, A., Aharonov, Y., and Imry, Y. Phase uncertainty and loss of interference: A general picture. Phys. Rev. A 41, 3436 (1990).

[196] Stickney, J. A., Anderson, D. Z., and Zozulya, A. A. Transistorlike behavior of a Bose-Einstein condensate in a triple-well potential. Phys. Rev. A 75, 013608 (2007).

[197] Stöckmann, H.-J. Quantum Chaos: an introduction. University Press, Cambridge (1999).

[198] Stöckmann, H.-J. and Schäfer, R. Fidelity Recovery in Chaotic Systems and the Debye-Waller Factor. Phys. Rev. Lett. 94, 244101 (2005).

[199] Switkes, M., Marcus, C. M., Campman, K., and Gossard, A. C. An adiabatic quantum electron pump. Science 283, 1905 (1999).

[200] Thouless, D. J. Quantization of particle transport. Phys. Rev. B 27, 6083 (1983).

[201] Treutlein, P., Hunger, D., Camerer, S., Hänsch, T. W., and Reichel, J. Bose-Einstein Condensate Coupled to a Nanomechanical Resonator on an Atom Chip. Phys. Rev. Lett. 99, 140403 (2007).

[202] Trombettoni, A. and Smerzi, A. Discrete Solitons and Breathers with Dilute BoseEinstein Condensates. Phys. Rev. Lett. 86, 2353 (2001).

[203] Tsironis, G. P. and Kenkre, V. M. Initial condition effects in the evolution of a nonlinear dimer. Phys. Lett. A 127, 209 (1988). 
[204] Atom traps worldwide, University of Innsbruck. http://www.uibk.ac.at/ exphys/ultracold/atomtraps.html (2007).

[205] Wang, Y.-J., Anderson, D. Z., Bright, V. M., Cornell, E. A., Diot, Q., Kishimoto, T., Prentiss, M., Saravanan, R. A., Segal, S. R., and Wu, S. Atom Michelson Interferometer on a Chip Using a Bose-Einstein Condensate. Phys. Rev. Lett. 94, 090405 (2005).

[206] Weiss, U. Quantum Dissipative Systems. World Scientific (1998).

[207] Wessel, S., Alet, F., Troyer, M., and Batrouni, G. G. Quantum Monte Carlo simulations of confined bosonic atoms in optical lattices. Phys. Rev. A 70, 053615 (2004).

[208] Wigner, E. P. Characteristic Vectors of Bordered Matrices with Infinite Dimensions. Ann. Math. 62, 548 (1955).

[209] Wigner, E. P. Characteristic Vectors of Bordered Matrices with Infinite Dimensions II. Ann. Math. 65, 203 (1957).

[210] Wilkinson, M. A semiclassical sum rule for matrix elements of classically chaotic systems. J. Phys. A 20, 2415 (1987).

[211] Wilkinson, M. Statistical aspects of dissipation by Landau-Zener transitions. J. Phys. A 21, 4021 (1988).

[212] Wilkinson, M. Parametric Random Matrices: Static and Dynamic Applications, pages 369-399. Kluwer Academic / Plenum Publishers, New York (1999).

[213] Wilkinson, M. and Austin, E. J. A random matrix model for the non-perturbative response of a complex quantum system. J. Phys. A 28, 2277 (1992).

[214] Wisniacki, D. A. and Cohen, D. Quantum irreversibility, perturbation independent decay, and the parametric theory of the local density of states. Phys. Rev. E 66, 046209 (2002).

[215] Wright, E., Eilbeck, J. C., Hays, M. H., Miller, P. D., and Scott, A. C. The quantum discrete self-trapping equation in the Hartree approximation. Physica D 69, 18 (1993).

[216] Wu, B. and Liu, J. Commutability between the Semiclassical and Adiabatic Limits. Phys. Rev. Lett. 96, 020405 (2006).

[217] Zwerger, W. Mott-Hubbard transition of cold atoms in optical lattices. J. Opt. B 5, S9 (2003). 


\section{Acknowledgments}

Many people have contributed in some way to this work, this thesis would not have been possible without them. I would like to use this opportunity to thank them here.

Theo Geisel created a work climate that is hardly found elsewhere. I sincerely thank him for giving me the opportunity to join his group and for the constant support I experienced from him.

I owe my deep gratitude to Tsampikos Kottos. His love for physics, his inspiring character, and his dedication have made the last years an invaluable experience. Looking back, I cannot remember a single moment where he would not offer help and advice. Thank you.

I would like to thank Doron Cohen for the long and fruitful collaboration and acknowledge the inspiring discussions with Andreas Buchleitner, Sergej Flach, George Kalosakas, Andrey Kolovsky, Tomaž Prosen, and Markus Oberthaler. Kurt Schönhammer is thanked for accepting to co-referee this thesis. I acknowledge the support of the GIF, the GermanIsraeli Foundation for Scientific Research and Development, the DFG via the Forschergruppe 760, and Wesleyan University.

All the members of our department contributed to an exceptional atmosphere which I will always keep in good memory. I would like to give a special thanks to Oliver Bendix and Antonio Méndez-Bermúdez with whom I spent a lot of time inside and outside the institute. I thank my office mates, Matthias Kaschube and Hecke Degering and my non-office mates Dirk Brockmann, Ragnar Fleischmann and Fred Wolf for their help and welcome distraction. Yorck-Fabian Beensen and Denny Fliegner are responsible for a computer environment that just works, which can be hardly overestimated. Concerning the challenges of administration, I must thank Katharina Jeremias, Corinna Trautsch and Regina Wunderlich for their help.

I must also mention the hospitality that was extended to me during my various visits at Wesleyan University and the support of the people at the physics department. Special thanks go to Anna Milardo, the good soul of the physics department, my office mate Steve Coppage, and Joshua Bodyfelt. Josh was never tired to discuss physics or help me in everyday life. Last but not least, I thank Frederik Süßmann and Kerem Alper for the welcome distraction during my visits. That was tropical.

The mensa crew, once an institution, had cheered me up on various occasions. Thanks to Karin, Maike, Leif, Stephie, and Sven. Thanks also to Frank van Bussel, Jörg Rampacher, and Katrina Smith-Mannschott for their help with the manuscript.

This thesis would not have been possible without the help of my family who has supported me during my entire studies. Thank you.

Finally, I have the deepest gratitude to Carola. Keule supported me and my plans at any instance of time and showed an unexpected level of tolerance. Thank you for everything. 


\section{Lebenslauf}

\section{Persönliche Daten}

Name: $\quad$ Moritz Hiller

Geburtsdatum: 20. Januar 1977

Geburtsort: Göttingen

Staatsangehörigkeit: deutsch

Familienstand: ledig

\section{Ausbildung}

Juni 1996

1997-2004

Oktober 1999

2000-2001

2002-2004

Januar 2004

2004-2007

2004-2007

2005-2007

\begin{abstract}
Abitur, Felix-Klein-Gymnasium, Göttingen
Diplomstudium Physik an der Georg-August-Universität, Göttingen
\end{abstract}

Vordiplom Physik

Erasmus Stipendium, Universidad de Sevilla, Spanien

Diplomand am Institut für Nichtlineare Dynamik, GeorgAugust-Universität, Göttingen, Titel der Arbeit: "Chaotic Quantum Dynamics, Irreversibility and the Applicability of Random Matrix Theory"

Diplom Physik

Promotionsstudium, Institut für Nichtlineare Dynamik, Georg-August-Universität, Göttingen

Wissenschaftlicher Mitarbeiter am Max Planck Institut für Dynamik und Selbstorganisation, Göttingen

Forschungsaufenthalte bei Prof. Dr. T. Kottos, Wesleyan University, CT, USA (Sep. 2005-Feb. 2006, Sep. 2006-

Dez. 2006, Mär. 2007-Mai 2007)

Göttingen, den 21.11.2007 